The Astrophysical Journal Supplement Series No. 231, 26:231-312

(C) 1973. The American Astronomical Society. All rights reserved. Printed in U.S.A.

\title{
THE EXPLOSIVE BURNING OF OXYGEN AND SILICON
}

\author{
S. E. Woosley \\ Rice University, Houston, Texas and Institute of Theoretical Astronomy, \\ Cambridge, England
}

W. DAVID ARNETT*

University of Texas at Austin and Institute of Theoretical Astronomy

AND

Donald D. Clayton

Rice University and Institute of Theoretical Astronomy

Received 1973 February 28

\begin{abstract}
This paper analyzes the explosive burning of oxygen and silicon. Major emphasis is on nuclear details of how the fuel burns and of the nucleosynthesis that results from the burning. By characterizing the explosions in terms of an $e$-folding time for the reduction of the density we are able to map the nature of the nucleosynthesis in terms of that time scale and the initial temperature, density, and chemical composition. These results make it possible in the future to infer rather well the results of explosive burning without carrying out the laborious integrations of the differential equations for the abundances, as we have done in this paper. The major and minor sections of the paper are as follows:
\end{abstract}

I. INTRODUCTION . . . . . . . . . . . . . . . . . . . . . . . . . . . .

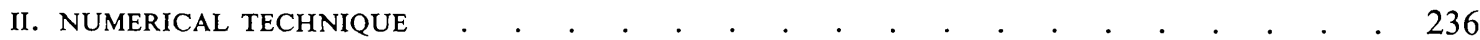

III. EXPLOSIVE OXYGEN BURNING . . . . . . . . . . . . . . . . . . . . . . . . . $\quad . \quad 239$

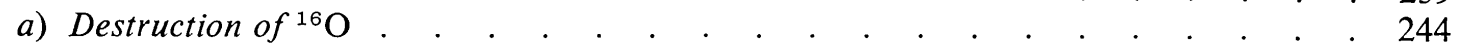

b) Destruction of ${ }^{12} \mathrm{C}$. . . . . . . . . . . . . . . . . . . . . . . . 244

c) Equations for $Y\left({ }^{16} \mathrm{O}\right)$ and $Y\left({ }^{12} \mathrm{C}\right)$.

d) Role of ${ }^{24} \mathrm{Mg}$. . . . . . . . . . . . . . . . . . . . . . . . . . . . .

e) Location of Excess Neutrons . . . . . . . . . . . . . . . . . . . . . . . 247

f) Free-Particle Exposures . . . . . . . . . . . . . . . . . . . . . . . . . . . .. .248

IV. QUASI-EQUILIBRIUM DURING EXPLOSIVE OXYGEN BURNING . . . . . . . . . . . $\quad 249$

a) Quasi-Equilibrium Clusters . . . . . . . . . . . . . . . . . . . . . . . 250

b) Freeze-out Corrections . . . . . . . . . . . . . . . . . . . 253

c) Temperature Required for Quasi-Equilibrium for $A \leq 45$. . . . . . . . . . . 256

d) Particle Densities at Freeze-out . . . . . . . . . . . . . . . . . . . . . 258

v. RESTRICTIONS ON CONDITIONS FOR EXPLOSIVE OXYGEN BURNING . . . . . . . . 263

a) Restrictions on Neutron Excess . . . . . . . . . . . . . . . . . . . . . . . . . . .

b) Restriction on the Alpha Excess . . . . . . . . . . . . . . . . . . 266

c) Abbreviated Reaction Network . . . . . . . . . . . . . . . . . . . . . . . . 270

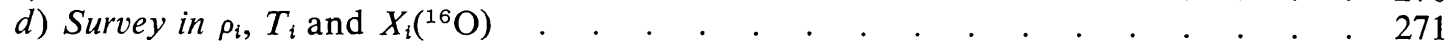

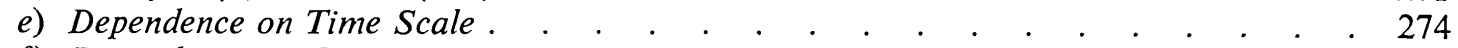

f) Dependence on Cross-Sections . . . . . . . . . . . . . . . . . . . . . . . . $\quad .274$

g) Combined ${ }^{12} \mathrm{C}$ and ${ }^{16} \mathrm{O}$ Explosions . . . . . . . . . . . . . . . . . . . . . . . . . . . 277

VI. SILICON BURNING . . . . . . . . . . . . . . . . . . . . . . . . 277

a) Merging of Explosive Oxygen Burning into Silicon Burning . . . . . . . . . . . 279

b) The Approach to Quasi-Equilibrium . . . . . . . . . . . . . . . . . . . . $\quad$.

c) Radioactive Progenitors . . . . . . . . . . . . . . . . . . . . . . . 286

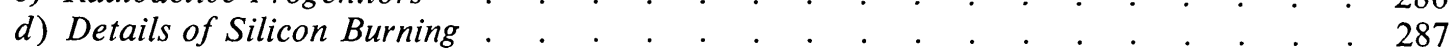

* Alfred P. Sloan Foundation Fellow. 
VII. SILICON EXHAUSTION AND NUCLEAR STATISTICAL EQUILIBRIUM $\quad . \quad$. . . . . $\quad$. 291

a) Normal Freeze-out . . . . . . . . . . . . . . . . . . . . . . . . . . 293

b) Alpha-rich Freeze-out . . . . . . . . . . . . . . . . . . 294

c) The Correct Neutron Excess . . . . . . . . . . . . . . . . . . . . . 297

VIII. CONCLUSIONS . . . . . . . . . . . . . . . . . . . . . . . . . 299

APPENDIX: THERMONUCLEAR REACTION RATES . . . . . . . . 303

Subject headings: abundances - nuclear reactions - supernovae

Symbol

\section{GLOSSARY OF TERMS}

$N_{n}(i) \quad$ Neutron number of nucleus $i$

Equation

$Z_{p}(i) \quad$ Proton number of nucleus $i$

Meaning

1

$Y(i) \quad$ Number density of species $i$ divided by the total number density of nucleons

$\eta \quad$ Excess neutrons per nucleon

$\rho \quad$ Number density of nucleons divided by Avogadro's number

$\rho_{i} \quad$ Initial density in the expansion

$\tau_{\mathrm{HD}} \quad$ Density time scale

$\chi \quad$ Time-scale factor

$T_{9} \quad$ Temperature in billions of degrees Kelvin

$T_{9 i} \quad$ Initial temperature in the expansion 3

$N_{\text {A }} \quad$ Avogadro's number

$X(i) \quad$ Fraction of nucleons in form of species $i$

$A(i) \quad$ Atomic number of species $i \quad 4$

$N(i) \quad$ Number density of species $i$

$\lambda_{j k}(i) \quad$ Thermally averaged rate per pair for $i+j \rightarrow k+l \quad 5,6$

$T_{9 f} \quad$ Freeze-out temperature $\quad 12$

$S_{k} \quad$ Exposure to free particles of type $k \quad 13$

$f_{j k ; i} \quad$ Forward flow from $i$ due to reaction $(j, k)$

$F_{j k ; i} \quad$ Net flow from or into $i$ due to $(j, k)$

$Y_{\mathrm{qe}}(i) \quad$ Quasi-equilibrium value of $Y(i)$

$C_{i}\left(T_{9}\right) \quad$ Quasi-equilibrium coefficient of species $i$

$r_{j} \quad \log _{10}\left[Y_{\mathrm{qe}}(j) / Y(j)\right]$

$\begin{array}{ll}\tau_{12} & \text { Time for } 2 \text { to equilibrate with } 1 \\ \delta_{f}(i) & \text { Logarithm of freeze-out correction for species } i\end{array}$

$\begin{array}{ll}\tau_{\mathrm{qe}} & \text { Time for establishment of quasi-equilibrium } \\ \delta_{n} & \eta \text { for restricted set }\{X\}_{\mathrm{FoB}}\end{array}$

$\begin{array}{lll}\delta_{n} & \eta \text { for restricted set }\{X\}_{\mathrm{EOB}} & 29 \\ \delta_{\alpha} & \text { Excess } \alpha \text {-particles per }{ }^{28} \mathrm{Si} \text { core in }\{X\}_{\mathrm{EOB}}\end{array}$

$\{X\}_{\mathrm{EOB}} \quad$ Set of nuclei synthesized primarily by explosive oxygen burning 30

$\eta_{\text {eff }} \quad$ Contribution of $\{X\}_{\text {EOB }}$ to total $\eta \quad 31$

$\triangle X_{16} \quad$ Oxygen mass fraction consumed

$f \quad$ Fraction of ${ }^{16} \mathrm{O}$ consumption via modes 1 and $2 \quad 34,39$

$\Delta X_{\alpha} \quad$ Alpha-mass-fraction excess 35,36

$X_{\mathrm{t}}\left({ }^{28} \mathrm{Si}\right) \quad$ Final mass fraction of total number of ${ }^{28} \mathrm{Si}$ cores $\quad 36,38$

$\phi \quad$ Initial ratio $X_{i}\left({ }^{24} \mathrm{Mg}\right) / X_{i}\left({ }^{28} \mathrm{Si}\right)$

$G_{1} \quad$ Abundance of set $28 \leq A \leq 45$

$G_{2} \quad$ Abundance of set $46 \leq A \leq 66$

$f_{1}^{45} \quad$ Fraction of $G_{1}$ as ${ }^{45} \mathrm{Sc}$

$f_{2}{ }^{46} \quad$ Fraction of $G_{2}$ as ${ }^{46} \mathrm{Ti}$

$\tau_{\min } \quad$ Theoretical minimum quasi-equilibration time 47

$t_{2} \quad$ Actual quasi-equilibration time (table 10) 47

${ }_{1}{ }_{9 \alpha} \quad$ Temperature where $\alpha$-reactions go out of equilibrium

$\S \mathrm{VII} a$

Condition on for alpha-rich freeze-out $\quad 67$

$t_{\alpha} \quad$ Time at $T_{9 \alpha}$ 


\section{INTRODUCTION}

It has long been a popular idea that the processes involved in the synthesis of elements in the intermediate mass range (say $28 \leq A \leq 62$ ) occur as normal part of the late evolutionary stages of a certain class of stars, namely those stars which become supernovae (or a sizable fraction thereof). This idea is particularly appealing as supernovae offer both a site for the high temperatures that are necessary for nuclear reactions with large Coulomb barriers and a very efficient means of dispersing the products of their alchemy into the interstellar medium. Unfortunately the complexity of the evolution, both structural and nuclear, has so far prohibited the exact tracking of the final evolutionary stages of such a highly evolved star except in a few special cases-for example the "12 C Detonation Model" of Arnett (Arnett 1969a; Arnett, Truran, and Woosley 1971, hereafter designated as ATW). Usually, simplifying approximations of one form or another have been employed to enable a calculation. The most common approximations, which are not mutually exclusive, are (1) equilibrium or quasi-equilibrium and (2) parametrized explosive expansions.

The equilibrium or quasi-equilibrium assumptions specify that all or a portion of the atomic nuclei have been exposed to high temperatures for a sufficiently long time so that the rates of all nuclear and electromagnetic reactions among the given set of species proceed at the same rate as the inverse reactions. Many researchers (e.g., Hoyle 1946; Burbidge et al. 1957; Fowler and Hoyle 1964) have pointed out the striking correlation of the abundances of the isotopes of iron, calculated under such conditions, with the observed natural abundances of these species. In their calculations a state of equilibrium was assumed to exist among all species. Then the abundance of each may be specified by values of the temperature, density, and neutron-excess parameter

$$
\eta \equiv \sum_{i}\left[N_{n}(i)-Z_{p}(i)\right] Y(i),
$$

where $N_{n}(i)$ and $Z_{p}(i)$ are the neutron and proton numbers of the species $i$ and $Y(i)$ is the number density of species $i$ divided by the total number density of nucleons. Physically, $\eta$ is the number of excess neutrons per nucleon in the gas. Good agreement with the natural abundances of the isotopes of iron was found to occur at a temperature of three to four billion degrees Kelvin $\left(T_{9}=3.0-4.0\right)$ and a density of several million grams per cubic centimeter $\left(\rho \sim 3.0 \times 10^{6}\right)$ if $\eta$ takes on values in a narrow range near 0.07 . More recently some success has been achieved in ascribing the synthesis of most of the abundant elements in the intermediate mass range to incomplete silicon burning. This idea, originally studied in the reaction-network calculations of Truran, Cameron, and Gilbert (1966), has been examined in detail by Bodansky, Clayton, and Fowler (1968; hereafter designated as BCF) and more recently by Michaud and Fowler (1972). Most of the abundant species in the mass range of interest can be produced by mass zones that have undergone silicon burning to various extents and achieved a state of equilibrium only for species in the mass range $28 \leq A \leq 62$ with respect to exchange of only the free protons, neutrons, photons, and alpha particles (quasi-equilibrium). A characteristic of these calculations is that a fairly low value of $\eta$ is required $(\eta \sim 0.002)$. This result differs markedly from earlier " $e$-process" calculations in that all $A=4 n$ nuclei are synthesized together along with an irongroup abundance peak centered on ${ }^{56} \mathrm{Ni}$ rather than ${ }^{56} \mathrm{Fe}$.

The shortcoming of the equilibrium approximation is inherent in its simplicity. Since so little information is required to compute an equilibrium distribution, very little information about the site of the synthesis can be inferred. All that one can demonstrate is that the solar abundances of the elements may have been produced by matter that at one time attained a particular state of equilibrium. Except, perhaps, for the value of $\eta$, information regarding the composition before the explosion and the 
thermodynamic conditions during the explosion is suppressed. Moreover, the comparison of abundances calculated in equilibrium, to actual abundances ejected from the explosion, must always contain some (hopefully small) error due to the processing that the elements must undergo while the temperature falls from the value at which the equilibrium distribution is calculated to zero.

For these reasons, researchers often prefer to employ parametrized explosive expansions and to follow explicitly the nuclear reactions that occur in a zone as it expands and cools. The calculation is dynamic and, because it requires additional hypotheses about the explosion, may yield useful information regarding the conditions prevailing during the synthesis. Also, this approach has the advantage of following the nuclear evolution to its conclusion when all nuclear interactions have ceased. Calculations of this nature have been performed for the explosive burning of carbon, of oxygen, and of silicon-rich compositions (Arnett 1969b; Truran and Arnett 1970, hereafter designated at TA70; Arnett and Clayton 1970; Truran and Arnett 1971) for specific parametrizations of the explosion (namely the peak density and temperature and the value of $\eta$ ) that are chosen to yield abundances of key species in accord with their naturally observed values. The overall agreement of abundant species in the mass range $16 \leq A \leq 62$ with their solar ratios is quite striking and suggests strongly that these species were indeed synthesized in such events.

Such calculations have thus far been primarily an attempt to show that explosive burning under certain select conditions can produce abundance distributions that correlate well with observations. Less emphasis has been placed on the generality of the solution (that is, for what range of explosive conditions can good correlation with natural abundances be achieved) and the manner in which the solution comes about (that is, the nuclear mechanisms which greatly affect the final distribution). The former is important as a means of delineating the conditions that we expect to have prevailed during the supernova explosions responsible for nucleosynthesis. The latter reveals which nuclear-reaction rates are important to the calculations and what error we may expect in the solution due to experimental errors in the key cross-sections. Also, no detailed attempt has been made to combine the two approaches (equilibrium and explosive) or to explain the dichotomy of solutions. For example, it is known that the abundances of most species in the mass range $28 \leq A \leq 42$ can be produced by a carefully chosen silicon quasi-equilibrium distribution which supposedly represents the results of explosive silicon burning in a series of zones which have burned only a small fraction of their initial silicon abundance (Michaud and Fowler 1972). But a solution of equally good correlation can result from a zone undergoing explosive oxygen burning (TA70) if the values of peak temperature and density are correctly chosen. On the surface these appear to be two very different processes, but the congruence of the results leads us to look for hidden similarities.

In our calculations we shall examine the possibility of synthesizing most of the abundant elements in the mass range $28 \leq A \leq 62$ by a combination of explosive oxygen burning and explosive silicon burning, or equivalently, as it will turn out, explosive carbon or oxygen burning at various values of peak temperatures. We are extending and unifying the work of Truran et al. (1966), BCF, TA70, Truran and Arnett (1971), and ATW. In particular, we shall be interested in understanding not only if it is possible to reproduce the observed abundances of these elements, but also the generality of the solutions. That is, we wish to determine the range of thermodynamic conditions under which agreement can be achieved and the implications for the model of the exploding object. We shall also point out similarities to previous calculations, especially those employing the quasi-equilibrium assumption, and why a correctly chosen quasi-equilibrium distribution may reasonably well approximate the results of explosive oxygen burning as well as silicon burning. The dependence of the results on certain key cross-section measurements will also be discussed. 
We take as our model a single stellar zone which is shock heated or flashed after degenerate ignition to some peak temperature $T_{9 i}$ (measured in billions of degrees Kelvin) and peak density $\rho_{i}$ (measured as number density of nucleons divided by Avogadro's number). We note that this number density is so nearly equal to the usual mass density that it may be so regarded in numerical results. The zone is then taken to cool adiabatically as it expands on a hydrodynamic time scale given approximately (Fowler and Vogl 1964; Fowler and Hoyle 1964; Arnett 1971) by

$$
\tau_{\mathrm{HD}}=446 \chi \rho_{i}{ }^{-1 / 2} \mathrm{~s},
$$

where $\chi$ is some arbitrary scaling parameter for testing the dependence of the results upon the time scale. Then the density and temperature at time $t$ are given by

$$
\begin{aligned}
\rho(t) & =\rho_{i} \exp \left(-t / \tau_{\mathrm{HD}}\right), \quad t>0, \\
T_{9}(t) & =T_{9 i}\left(\frac{\rho}{\rho_{i}}\right)^{\gamma-1},
\end{aligned}
$$

where $\gamma$ is the adiabatic exponent (taken equal to $4 / 3$ in these calculations). Once $T_{9 i}$ and $\rho_{i}$ are specified the thermodynamic history of the zone is given by these approximations. For a given set of initial abundances and nuclear reaction rates the final abundance distribution is also uniquely specified. The initial abundances are usually taken to represent the products of previous hydrostatic burning phases in the zone. Equation (3) implicitly assumes that the rise in temperature and density to their peak values is instantaneous or, more exactly, that energy is deposited in the zone in a time shorter than the time scale for important nuclear reactions; that is, $\tau_{\text {rise }} \ll \tau_{\text {nuc }}$. Here we will be interested only in nuclear evolutions that process their initial composition significantly, hence we will require $\tau_{\text {nuc }} \leqslant \tau_{\text {HD }}$. Thus the assumption of instantaneous rise time really means that the energy must be dumped into the zone in a time short compared to the local hydrodynamic time scale. Due to the high-temperature dependence of the important nuclear reaction rates, the error in $T_{9 i}$ should be small (amounting to no more than a few percent even if $\tau_{\text {rise }}=\tau_{\text {HD }}$ ). Since the initial compositions which we shall employ for explosive oxygen and silicon burning are quite uncertain anyway, this error is negligible at present. Stellar evolutionary calculations are currently underway which should reduce these uncertainties.

The results of these calculations should represent the nucleosynthesis that occurs in stellar zones for a range of peak temperatures and densities such that $\rho_{i}>10^{4}$ and $T_{9 i}>3.0$. No assumptions about the supernova model other than equations (2) and (3) are included; therefore the results are applicable to any material which undergoes sudden heating and adiabatic expansion on roughly a hydrodynamic time scale. We do not specify that these zones be individual zones of a single parent supernova. They may in fact represent typical zones in a variety of supernovae. When comparing to observed interstellar abundances what matters is the total ensemble of exploding zones in the past history of the galaxy and the mixing that has occurred. Such considerations are beyond the present scope of this paper; see Arnett (1971), ATW, and Talbot and Arnett (1971). However, the conditions which we will find here to be appropriate to the synthesis of nuclei should be representative of a "typical" zone out of which the given species arose. Though zones may exist with conditions quite different from those we shall derive, we would expect their weight in the canonical integration that has occurred over galactic history to be small.

In order to evaluate these calculations it is necessary to choose a comparison set of "natural" abundances which represent at least approximately the composition of the interstellar medium in the Sun's vicinity. The abundances to be employed here are primarily those of Cameron (1968). The abundances of ${ }^{36} \mathrm{Ar}$ and ${ }^{38} \mathrm{Ar}$ have been 
reduced by a factor of 2 from Cameron's value to account for recent results from lunar samples by Eberhardt et al. (1970). The meteoritic value for the abundance of iron has been employed rather than the solar photospheric value which, until recently, was believed to be a factor of 5 smaller. Recent measurements of the pertinent oscillator strengths ( $f$-values) made by Garz and Kock (1969), Grasdalen, Huber, and Parkinson (1969), Huber and Tobey (1968), and Whaling, King, and Martinez-Garcia (1969) have shown that the photospheric value may be brought into agreement with the meteoritic value.

The results obtained here will also depend somewhat upon the choice of nuclear cross-sections. The values to be employed in these calculations are given in the Appendix. It will be the philosophy of this discussion to assume those values to be correct and later to examine the dependence of the results upon the few cross-sections that are found to be critical. The system contains some inherent compensation. Often it will happen that reactions come into approximate equilibrium with their inverses. In such cases the actual cross-section makes little difference, the abundances being determined instead by experimental binding energies that are accurately known. Thus the results will often be much more accurately determined than the cross-sections employed.

\section{NUMERICAL TECHNIQUE}

In order to follow the changes in composition which occur when matter is subjected to transformation by thermonuclear reactions at very high temperatures it is necessary to solve a large set of coupled differential equations. Because the equations are nonlinear, the solution must be accomplished by a numerical technique. The number density $N(i)$ of species $i$ can be written

$$
N(i)=\rho N_{\mathrm{A}} X(i) / A(i) \equiv \rho N_{\mathrm{A}} Y(i)
$$

where $X(i)$ and $A(i)$ are, respectively, the nucleon fraction (number of nucleons bound in the species $i$ divided by the total number of nucleons) and atomic number of species $i$, and $N_{\mathrm{A}}$ is Avogadro's number. The number density of species $i$ divided by the total number density of nucleons, which we designate by $Y(i)$ in equation (4), is a very useful abundance variable for the differential equations. In a system having homogeneous composition, or in an inhomogeneous system where chemical mixing is omitted, the number densities $N(i)$ change in time due to nuclear reactions and due to the density change in the expansion. However, in the corresponding differential equation for the time evolution of $Y(i)$ the rate of change of the density does not appear and we obtain an equation involving only nuclear reactions; viz.,

$$
\frac{d Y(i)}{d t}=-\sum_{j, k} Y(i) Y(j) \lambda_{j k}(i)+\sum_{l, m} Y(l) Y(m) \lambda_{m i}(l),
$$

where

$$
\lambda_{j k}(i) \equiv \rho N_{\mathrm{A}}\left\langle\sigma_{i} v\right\rangle_{j k}
$$

and $\left\langle\sigma_{i} v\right\rangle_{j k}$ is the thermally averaged product of cross-section times relative velocity for an interaction involving a particle of species $j$, incident upon a target of species $i$, giving a particle $k$ in the exit channel. The concepts "incident particle" and "target" and the notation defined here are, in spite of their obvious asymmetries, useful in that the rate of destruction of ${ }^{28} \mathrm{Si}$, say, by radiative interactions with protons will be written $\lambda_{p y}\left({ }^{28} \mathrm{Si}\right)$, which we will adopt without further explanation in spite of the fact it could equally well be written $\lambda^{28} \mathrm{Si}^{29} \mathrm{P}(p)$. The sum over $l$ and $m$ includes all those reactions which lead directly to the production of the species $i$. In this discussion it will be assumed that each bound species may undergo up to 15 interactions. These will include all the strong binary interactions involving a neutron, proton, $\alpha$-particle, or photon in an 
entrance or exit channel as well as the weak interactions: beta decay, electron capture, and positron emission. In addition, $\alpha$-particles, ${ }^{12} \mathrm{C}$, and ${ }^{16} \mathrm{O}$ may undergo triplealpha, ${ }^{12} \mathrm{C}+{ }^{12} \mathrm{C},{ }^{12} \mathrm{C}+{ }^{16} \mathrm{O}$, and ${ }^{16} \mathrm{O}+{ }^{16} \mathrm{O}$.

A set of differential equations having the form of equation (5) coupled by these reactions is called a "reaction network." The choice of species to be included in the network will be determined by the conditions of the nuclear burning. Generally the higher the burning temperature the more species one must include in the network. Two such networks are shown in figures $1 a$ and $1 b$. Figure $1 a$ shows the network which we will employ in our examination of explosive oxygen burning. The network in figure $1 b$ will be used at higher temperatures for explosive silicon burning and the $e$-process. The largest network that will be employed in these calculations will contain approximately 100 species and more than 400 reaction links; i.e., 400 forward reactions, 400 inverse reactions; and 140 weak interactions. Obviously an analytic solution of equation (5) becomes, for practical purposes, impossible for such a complex network. The coupled equations must be solved numerically. The method of solution which we shall employ here was originally discussed by Arnett and Truran (1969). Let the change in the abundance $Y(i)$ over a finite time interval $\Delta t=t^{n+1}-t^{n}$ be denoted $\Delta_{i}=Y^{n+1}(i)-Y^{n}(i)$. Then, taking $d Y(i) / d t \simeq \Delta_{i} / \Delta t$ and neglecting quadratic terms in $\Delta_{i}$, the set of equation (5) may be transformed into the linearized set

$$
\begin{aligned}
& Y^{n+1}(i)\left[\frac{1}{\Delta t}+Y^{n}(j) \lambda_{j k}{ }^{n+1}(i)\right]+Y^{n+1}(j)\left[Y^{n}(i) \lambda_{j k}{ }^{n+1}(i)\right]-Y^{n+1}(l)\left[Y^{n}(m) \lambda_{m r}{ }^{n+1}(l)\right] \\
&-Y^{n+1}(m)\left[Y^{n}(l) \lambda_{m r}{ }^{n+1}(l)\right] \\
&=Y^{n}(i) Y^{n}(j) \lambda_{j k}{ }^{n+1}(i)-Y^{n}(l) Y^{n}(m) \lambda_{m r}{ }^{n+1}(l)+Y^{n}(i)\left(\frac{1}{\Delta t}\right) \cdot(7)
\end{aligned}
$$

This coupled set of linear equations may be solved by matrix inversion to give the updated abundances $Y^{n+1}(i)$ at the time $t^{n+1}$.

The size of the time step to be employed in equation (7) is limited by the demand that omitted quadratic terms in $\Delta_{i}$ are small enough to be negligible. This is equivalent to the demand that $\Delta_{i} / Y(i) \ll 1$ for any time step. This criterion should be satisfied if

$$
\Delta t \ll\left[\frac{d Y(i)}{d t}\right]^{-1} Y(i)
$$

for all important species $i$, or, defining a parameter $\epsilon$ by

$$
\Delta t \equiv \epsilon\left[\frac{d Y(i)}{d t}\right]^{-1} Y(i),
$$

if $\epsilon \ll 1$. The derivative is evaluated over the last time step. The time step at $t^{n+1}$ will be numerically selected in terms of the previous time step by the condition

$$
\Delta t^{n+1}=\epsilon \min \left[\frac{Y^{n+1}(i)}{\Delta_{i}}\right] \Delta t^{n},
$$

and by specifying a tolerably small value of $\epsilon$. In the actual calculations performed here only abundances having $Y(i) \geq 10^{-8}$ were allowed to participate in equation (8). The value of $\epsilon$ employed was 0.15 . Also, to prevent inordinately long time steps from occurring when abundances are changing very slowly, the new time step was never allowed to be more than twice as long as the previous time step. A second restriction on the time step should be that the $\lambda_{j k}{ }^{n+1}$ change little over $\Delta t$. The criteria above are usually adequate, at least for the important reactions. 
(a)

Reaction Network

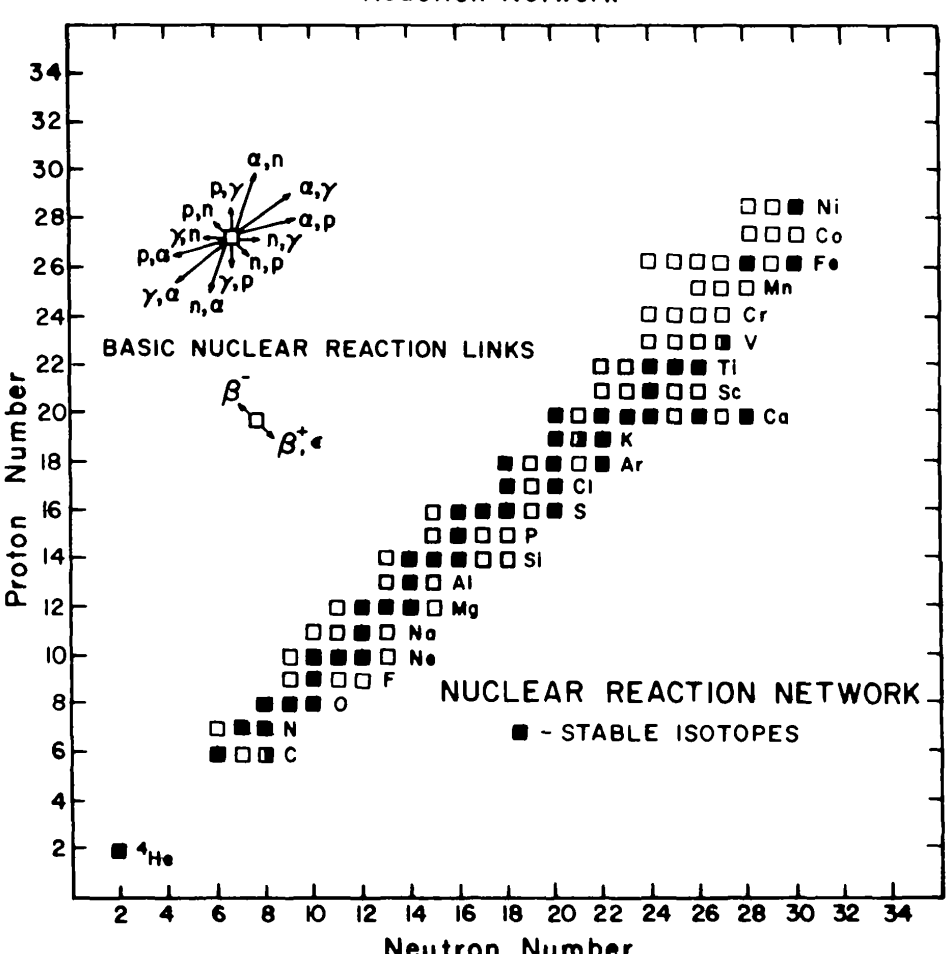

(b)

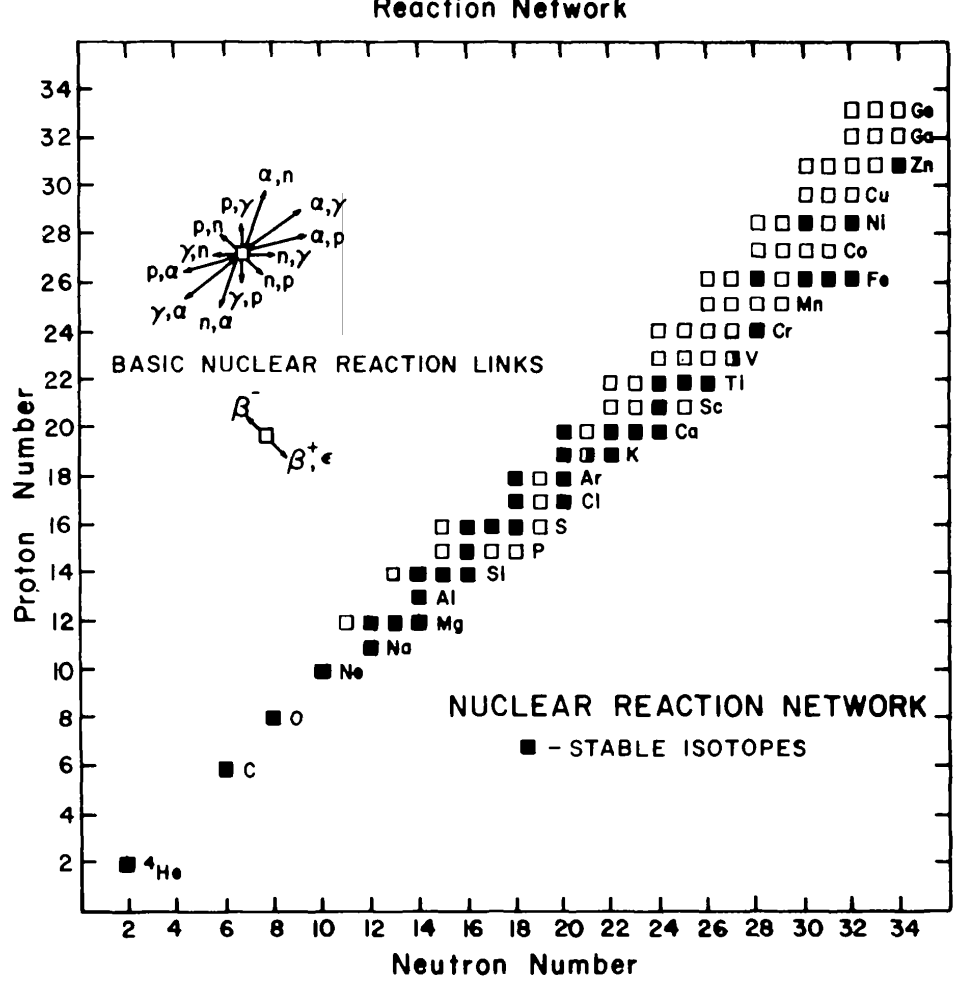

FIGS, $1 a$ and $1 b$.-Two typical reaction networks. Terrestrially stable nuclei are represented by solid squares and unstable nuclei by open squares except for ${ }^{14} \mathrm{C},{ }^{40} \mathrm{~K}$, and ${ }^{50} \mathrm{~V}$, which having astronomically long half-lives, are represented by half-filled squares. These nuclei are coupled by all possible reactions of the type illustrated. Also included, but not illustrated, are links due to the $3 \alpha$ reaction and the various branches of ${ }^{12} \mathrm{C}+{ }^{12} \mathrm{C},{ }^{12} \mathrm{C}+{ }^{16} \mathrm{O}$, and ${ }^{16} \mathrm{O}+{ }^{16} \mathrm{O}$. 
The criterion that abundances as small as $10^{-8}$ change by no more than 15 percent in a given time step results in much slower changes in the larger abundances. Often several hundred time steps were necessary to change the abundance of primary species by a factor of 2 . Thus the errors in the final results for large abundances due to the finite size of time steps should be small. We estimate that no species of nonnegligible final abundance is in error by more than five percent due to the finite size of time steps. This amount of error is usually negligible in comparison with the error due to the uncertainty in the cross-sections.

The cross-sections employed in these calculations are given in the Appendix and contain many new experimental measurements reflecting the current state of the data analysis. Experimentally measured binding energies adopted from Mattauch, Thiele, and Wapstra (1965) have been employed for all species except ${ }^{64} \mathrm{Ge}$ for which no experimental value was available. Previous calculations have often used reaction " $Q$-values" which were calculated from mass-law formulae. Because these $Q$-values are very important to reactions where approximate equilibration of forward and reverse flows is attained, the experimental values, though only slightly different, have been employed. Weak-interaction rates calculated by Hansen (1968) have been incorporated into the network for all species so that nuclear evolutions may be followed through regions of changing neutron excess.

One of the most useful advances in technique has been the development of a "variable-reaction network." In the past computer codes of this type have been of the "fixed network" type. The species included in the set of equation (5) have generally been unalterable without some rewriting of the computer program. This inflexibility has made exploration of a new region of the periodic chart difficult. With the new form, cross-section information is read in for whatever elements are desired to be incorporated in the network. The computer program then automatically sets up the linkages appropriate to coupling these elements into a network. This flexibility allows instantaneous modification of the network to the form most suitable for a given calculation, resulting in nuclear evolutions that are speedier and more accurate.

Usually a given run will require from 300 to 600 time steps for completion. The computer time required for a single time step will vary with the size of the network and, of course, with the type of machine being used. On a CDC-6600 using single-precision arithmetic (14 digits) the computer time required for one nuclear time step for a network containing $N$ species was approximately $t=8.0 \times 10^{-3} \mathrm{~N}$ s. Most of this time is spent in inverting the matrix given by equation (7). Since, for the most part, this matrix is of a tridiagonal form, the time for inversion of the matrix is roughly proportional to $N$ rather than $N^{2}$ (for reasonably large values of $N$ ) as is often the case for a more general matrix. (We use a Gaussian eliminator algorithm with specially modified logic.) The total number of time steps required for a given evolution also depends somewhat upon $N$, since there are more species to be included in equation (8) resulting in time steps that are somewhat smaller.

\section{EXPLOSIVE OXYGEN BURNING}

The idea that the solar abundances of the elements between silicon and calcium might be reproduced by the explosive processing of oxygen-rich stellar zones was first investigated by TA70. They found the first detailed agreement with solar-system abundances for isotopic ratios in the range $28 \leqslant A \leqslant 42$; this was also the first calculation to seriously consider the effects of freeze-out on nucleosynthesis of these nuclei. They further pointed out that: (1) explosive oxygen burning exhibited a quasiequilibrium character, but with the net production of $\alpha$-particles and nucleons determined in a complex way by ${ }^{16} \mathrm{O}+{ }^{16} \mathrm{O},{ }^{12} \mathrm{C}+{ }^{12} \mathrm{C}$, and various photodisintegration and capture reactions on light nuclei $(A<28),(2)$ as in explosive carbon burning, 
good abundance agreement resulted only for a small range of neutron excess $(\eta \sim$ 0.002 ), and (3) good abundance agreement resulted only for a limited range of peak temperature and density. The density limitation is especially important because explosive carbon burning and silicon burning are relatively insensitive to density.

In view of the success of these preliminary calculations, a more detailed investigation and analysis is needed. We shall attempt in this paper to give a more generalized discussion of the nature of explosive oxygen burning - how and why agreement with solar values is obtained; for what range of compositions and explosive parameters agreement may be expected; and why a correctly chosen quasi-equilibrium distribution can be expected to approximate closely the results of explosive oxygen burning. Moreover, since there has been considerable progress in the determination of nuclear reaction rates under conditions appropriate to explosive nucleosynthesis in the last few years, the updating of reaction rates as well as the inclusion of previously neglected nuclear processes of import during explosive burning is highly desirable. Two such previously neglected reactions are ${ }^{12} \mathrm{C}+{ }^{16} \mathrm{O}$ and ${ }^{16} \mathrm{O}(p, \alpha)^{13} \mathrm{~N}$. We find that the inclusion of both of these can significantly alter the nucleosynthesis that occurs in a zone expanding from a given peak temperature and density. The reaction ${ }^{12} \mathrm{C}+{ }^{16} \mathrm{O}$ has been recently discussed in the literature (Woosley, Arnett, and Clayton 1971; Patterson et al. 1971) and seems fairly well determined. The reaction ${ }^{16} \mathrm{O}(p, \alpha)^{13} \mathrm{~N}$ has also been discussed (Woosley et al. 1972a), and the rate for the reaction is currently regarded with some skepticism. Indeed the uncertainty in this reaction rate is one of the most important experimental uncertainties involved in these calculations. The value erîployed here is due to Wagoner (1969) and is probably an overestimate. In a later section we will show the effect of varying this and other important cross-sections.

The nuclear-reaction network employed for these calculations was shown in figure 1a. It differs from the network of TA70 in that the nuclear detail below ${ }^{28} \mathrm{Si}$ is much more accurately represented. This was done so that no reaction would be overlooked which might be important in the destruction of the ${ }^{16} \mathrm{O}$ fuel. Due to the flexible nature of the network, elements which did not turn out to form important links could be deleted easily. The initial composition employed for these early test calculations was the same as employed by TA70. The zone under consideration was assumed to have undergone hydrostatic carbon burning with an initial composition of about half ${ }^{16} \mathrm{O}$ and half ${ }^{12} \mathrm{C}$. After sufficient time has elapsed so that the ${ }^{20} \mathrm{Ne}$ created during that phase has been photodisintegrated, a representative composition by mass fraction should be

$$
\begin{array}{ll}
X_{i}\left({ }^{16} \mathrm{O}\right)=0.54, & X_{i}\left({ }^{26} \mathrm{Mg}\right)=0.02, \\
X_{i}\left({ }^{24} \mathrm{Mg}\right)=0.30, & X_{i}\left({ }^{28} \mathrm{Si}\right)=0.14 .
\end{array}
$$

Explosive burning of this composition according to the prescriptions in equations (2) and (3) with $T_{9 i}=3.6, \rho_{i}=5.0 \times 10^{5}$, and $\chi=1.0^{-}$(the conditions of "best fit" for TA70) did not give good agreement with the solar abundances in the silicon-tocalcium region. In particular, species such as ${ }^{36} \mathrm{Ar},{ }^{38} \mathrm{Ar},{ }^{39} \mathrm{~K},{ }^{41} \mathrm{~K}$, and ${ }^{40} \mathrm{Ca}$, are grossly underproduced relative to ${ }^{28} \mathrm{Si}$. The reason for this discrepancy with the results of TA70 is the inclusion of new rates, in particular the reaction ${ }^{12} \mathrm{C}+{ }^{16} \mathrm{O}$, in this present calculation. The mechanics of this reaction and why it results in the diminished production of heavier species (relative to ${ }^{28} \mathrm{Si}$ ) will be discussed in greater detail in a later section. We will adopt the initial composition of equation (9) as a standard or reference composition in much that follows.

A second calculation which employed the same conditions and composition except that the peak density was lowered slightly (from $5.0 \times 10^{5} \mathrm{~g} \mathrm{~cm}^{-3}$ to $2.0 \times 10^{5} \mathrm{~g}$ $\mathrm{cm}^{-3}$ ) did succeed in reproducing the major abundances from ${ }^{28} \mathrm{Si}$ to ${ }^{42} \mathrm{Ca}$, as well as 


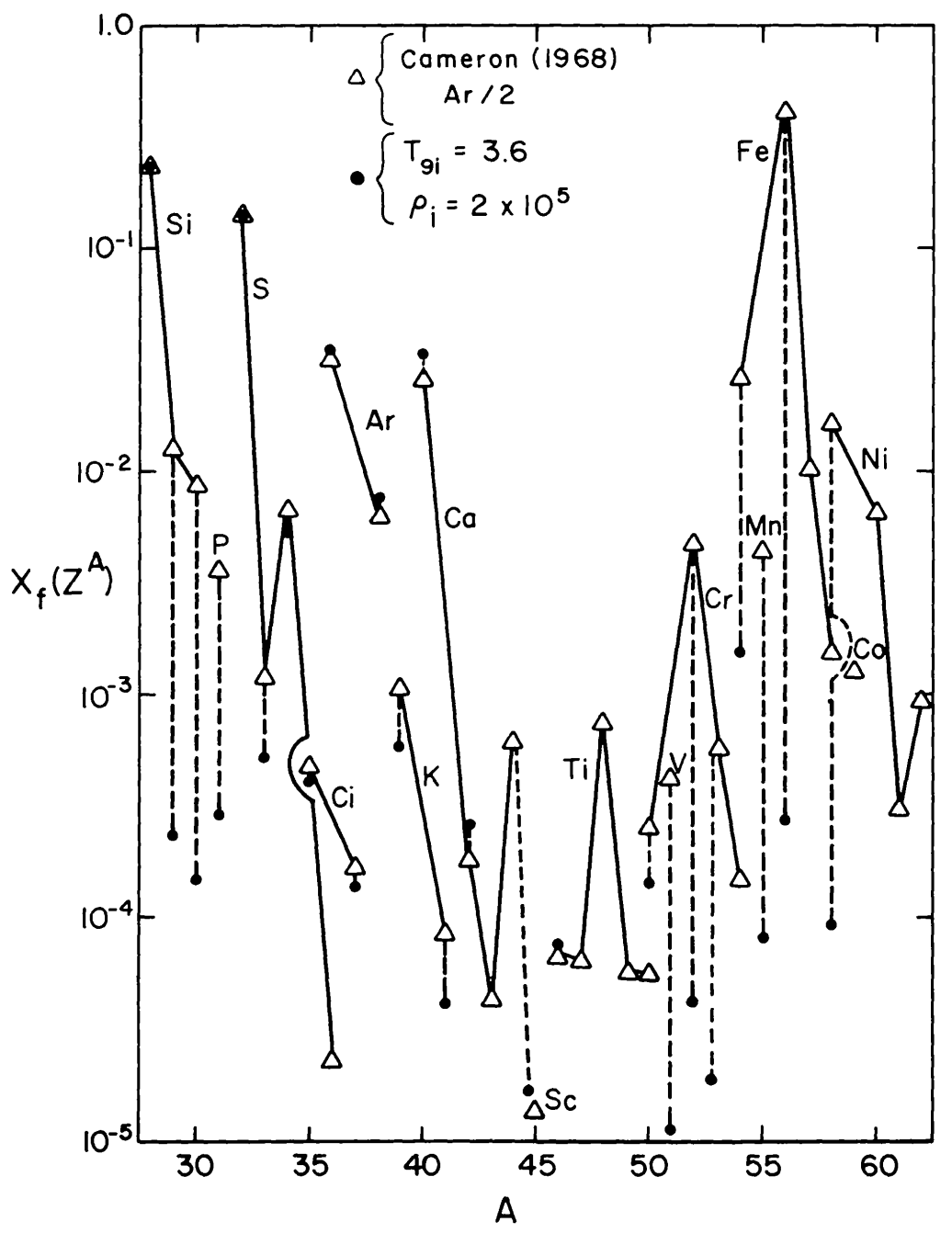

FIG. 2.-Nucleosynthesis results of explosive oxygen burning. The final mass fraction of the species $Z^{A}$ is shown compared to its solar value for an explosive-oxygen-burning run which employed a peak temperature of $T_{9 i}=3.6$ and density $\rho_{i}=2.0 \times 10^{5}$. The results have been normalized to ${ }^{28} \mathrm{Si}$. The abundances shown are those of the most abundant isobar of the given species which would decay to that species after ejection. Mass fractions smaller than $10^{-5}$ are not indicated.

producing the correct solar abundances of ${ }^{46} \mathrm{Ti}$ and ${ }^{50} \mathrm{Cr}$. The results are shown in figure 2, and are in substantial agreement with those of TA70 although obtained under explosive conditions that were slightly different. This is an important effect (and will be explained in a later section when we discuss the quasi-equilibrium nature of explosive oxygen burning). It appears that a change in one or several of the explosive parameters (cross-sections, composition, peak conditions, and time scale) can usually be compensated for by a shift in one or several of the other explosive parameters so that the same good fit shown in figure 2 is obtained. In this case, the change in nuclear evolution brought about by changing the ${ }^{12} \mathrm{C}+{ }^{16} \mathrm{O}$ rate from zero to the value given in the Appendix was compensated for by adjusting the peak density to a lower value. This observation indicates that there is most likely a multiplicity of explosive-oxygen-burning solutions to the solar distribution from silicon to calcium involving related sets of explosive parameters. The determination of these parametric relations will be discussed in detail in a later section. 


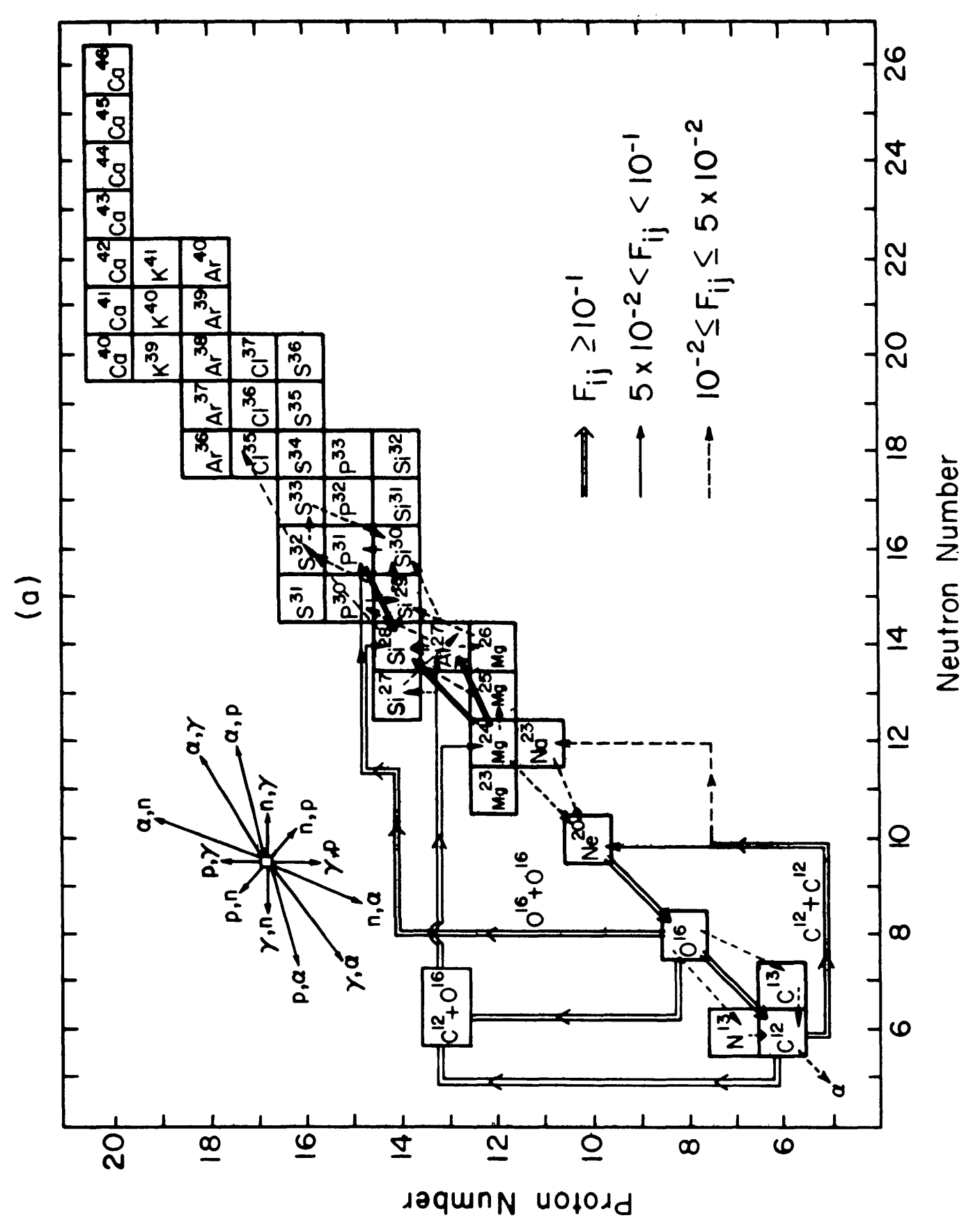

242 
(b)

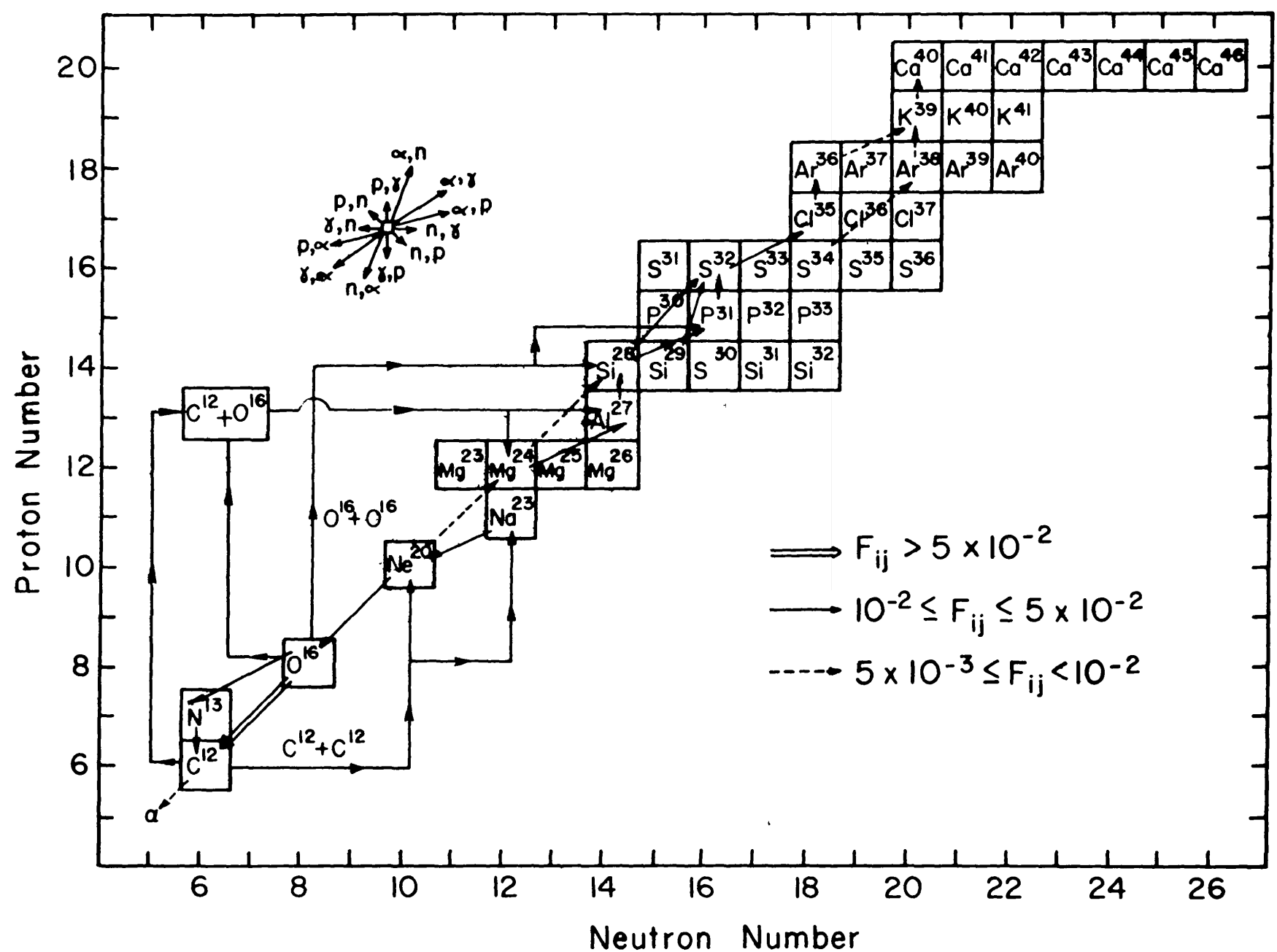

Frgs. $3 a$ and $3 b$.-Primary flows during explosive oxygen burning. Principal flows of the strength and type illustrated are shown at two times during the "standard" explosive-oxygen-burning run $\left(T_{91}=3.6, \rho_{4}=2.0 \times\right.$ $\left.10^{5}\right)$. Fig. $3 a$ is a "snapshot" at time $7.53 \times 10^{-3} \mathrm{~s}$ at which point $X\left({ }^{24} \mathrm{Mg}\right)=0.18$ and $X\left({ }^{16} \mathrm{O}\right)=0.49$. Fig. $3 b$ shows flows at time $9.01 \times 10^{-2} \mathrm{~s}$ at which point $X\left({ }^{24} \mathrm{Mg}\right)=2.89 \times 10^{-4}$ and $X\left({ }^{16} \mathrm{O}\right)=0.25$ 


\section{a) Destruction of ${ }^{16} \mathrm{O}$}

We now turn to an examination of the major nuclear flows that characterize what occurs. The important flows are shown in figures $3 a$ and $3 b$ for two different elapsed times into the evolution. Figure $3 a$ shows the character of the flows at a point early in the evolution before the initial ${ }^{24} \mathrm{Mg}$ abundance has been depleted. Figure $3 b$ shows the situation after the ${ }^{24} \mathrm{Mg}$ abundances has fallen to a very small value. The principal reaction in both cases is the direct interaction of two ${ }^{16} \mathrm{O}$ nuclei to produce compound nuclear states of ${ }^{32} \mathrm{~S}$ which we assume may decay through any of the four channels

$$
\begin{aligned}
{ }^{16} \mathrm{O}+{ }^{16} \mathrm{O} \rightarrow\left({ }^{32} \mathrm{~S}\right)^{*} & \rightarrow{ }^{28} \mathrm{Si}+\alpha+9.59 \mathrm{MeV}, \quad 34 \text { percent }, \\
& \rightarrow{ }^{31} \mathrm{P}+p+7.68 \mathrm{MeV}, \quad 56 \text { percent }, \\
& \rightarrow{ }^{31} \mathrm{~S}+n+1.45 \mathrm{MeV}, \quad 5 \text { percent }, \\
& \rightarrow{ }^{30} \mathrm{P}+d-2.41 \mathrm{MeV}, \quad 5 \text { percent },
\end{aligned}
$$

where the branching ratios employed are those adopted by TA70 and represent approximately the data of Spinka and Winkler (1972). We also see several other strong flows that are destroying ${ }^{16} \mathrm{O}$. These are high-temperature processes which are not normally encountered when oxygen burns hydrostatically but become important only when the temperature must be sufficiently high to burn a sizable fraction of ${ }^{16} \mathrm{O}$ in approximately a hydrodynamic time scale. Most involve the reaction of an ${ }^{16} \mathrm{O}$ nucleus with a free particle (neutron, proton, or photon) and lead to the production of a ${ }^{12} \mathrm{C}$ nucleus with the net release of an $\alpha$-particle. The most important of these is the direct photodisintegration of an ${ }^{16} \mathrm{O}$ nucleus to form ${ }^{12} \mathrm{C}$ and an $\alpha$-particle,

$$
{ }^{16} \mathrm{O}+\gamma \rightarrow{ }^{12} \mathrm{C}+\alpha-7.16 \mathrm{MeV} \text {. }
$$

It should be pointed out that at explosive-oxygen-burning temperatures $\left(3.0 \leqslant T_{9} \leqslant\right.$ 4.0) this reaction occurs mainly through resonances higher in the ${ }^{16} \mathrm{O}$ compound nucleus than the $7.12 \mathrm{MeV}$ level for which the reduced alpha width is currently regarded with great uncertainty. Thus, although the rate for the ${ }^{12} \mathrm{C}(\alpha, \gamma)^{16} \mathrm{O}$ reaction at low temperature (e.g., hydrostatic helium burning) may be very uncertain, the rate given in the Appendix for the above reaction is probably accurate to within a factor of 2. Higher temperature and lower density favor this process over the direct combination of two ${ }^{16} \mathrm{O}$ nuclei. An ${ }^{16} \mathrm{O}$ nucleus may also interact with protons and, to a lesser extent, with neutrons, to produce ${ }^{12} \mathrm{C}$ by ${ }^{16} \mathrm{O}(p, \alpha){ }^{13} \mathrm{~N}(\gamma, p){ }^{12} \mathrm{C}$ or ${ }^{16} \mathrm{O}(n, \alpha){ }^{13} \mathrm{C}(\gamma, n)^{12} \mathrm{C}$. These reactions are also favored by higher temperature and lower density. In figure 4 are shown those regions in the peak temperature-density plane where one or the other of the three processes, ${ }^{16} \mathrm{O}+{ }^{16} \mathrm{O},{ }^{16} \mathrm{O}(\gamma, \alpha){ }^{12} \mathrm{C}$, or ${ }^{16} \mathrm{O}(p, \alpha){ }^{13} \mathrm{~N}$, can be expected to dominate in the destruction of ${ }^{16} \mathrm{O}$ (during the course of an adiabatic expansion on a hydrodynamic time scale). The plot was obtained by surveying with a very abbreviated network discussed in $\S \mathrm{V} c$ and employing the initial composition given in equation (9). Dotted lines indicate the locus of points for which the dominant reaction burns twice as much ${ }^{16} \mathrm{O}$ as the nearest competing reaction. All of the ${ }^{13} \mathrm{C}$ and ${ }^{13} \mathrm{~N}$ produced by the reaction of ${ }^{16} \mathrm{O}$ with protons and neutrons are immediately photodisintegrated to ${ }^{12} \mathrm{C}$ so that the net result is an enhancement of ${ }^{16} \mathrm{O}(\gamma, \alpha)^{12} \mathrm{C}$. Thus at low densities $\left(\rho_{i} \leqslant 10^{6}\right)$, a sizable fraction of the ${ }^{16} \mathrm{O}$ that burns is converted into ${ }^{12} \mathrm{C}$ and an $\alpha-$ particle. Other reactions involving ${ }^{16} \mathrm{O}$ and free particles are negligible.

\section{b) Destruction of ${ }^{12} \mathrm{C}$}

Given an efficient mechanism for the production of ${ }^{12} \mathrm{C}$ one must inquire as to its fate. From figures $3 a$ and $3 b$ we can see that the ${ }^{12} \mathrm{C}$ is destroyed chiefly by reactions with itself and with ${ }^{16} \mathrm{O}$ nuclei. A small amount of the ${ }^{12} \mathrm{C}$ may be photodisintegrated into $\alpha$-particles. Other reactions involving ${ }^{12} \mathrm{C}$ and free particles are negligible, the 


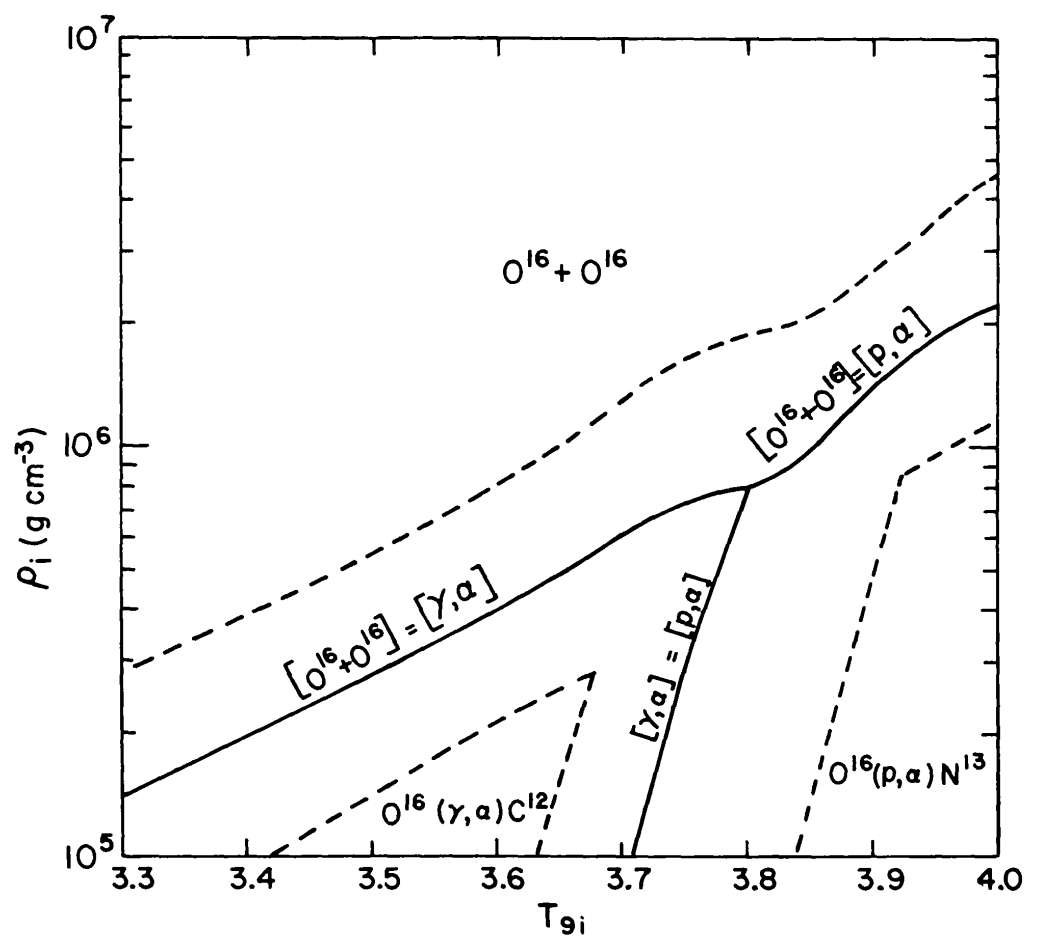

FIG. 4.-Dominant means of destruction of ${ }^{16} \mathrm{O}$ for various initial conditions. Regions in the initial temperature-density plane where one of the three reactions ${ }^{16} \mathrm{O}+{ }^{16} \mathrm{O},{ }^{16} \mathrm{O}(\gamma, \alpha)^{12} \mathrm{C}$, or ${ }^{16} \mathrm{O}(p, \alpha)^{13} \mathrm{~N}$ predominate in destroying ${ }^{16} \mathrm{O}$ during the course of the explosion are delimited. Solid lines indicate the locus of initial conditions that will lead to equal mass fractions of ${ }^{16} \mathrm{O}$ being consumed by the two indicated reactions during the explosion. Dotted lines indicate the locus of points for which the indicated reaction burns twice as much ${ }^{16} \mathrm{O}$ as the reaction of the neighboring domain. This figure was prepared using the initial composition given in eq. (9), but the nature of the delimitation does not vary much for other initial compositions containing sizable fractions of ${ }^{16} \mathrm{O}$.

flow out of ${ }^{12} \mathrm{C}$ by the forward reaction being overshadowed by the flow into ${ }^{12} \mathrm{C}$ by the inverse reaction either directly or indirectly from ${ }^{16} \mathrm{O}$. Thus the chief means of destruction of ${ }^{12} \mathrm{C}$ are the reactions

$$
\begin{aligned}
{ }^{12} \mathrm{C}+{ }^{12} \mathrm{C} \rightarrow\left({ }^{24} \mathrm{Mg}\right)^{*} & \rightarrow{ }^{23} \mathrm{Na}+p+2.24 \mathrm{MeV}, \quad 32 \text { percent }, \\
& \rightarrow{ }^{20} \mathrm{Ne}+\alpha+4.62 \mathrm{MeV}, \quad 66 \text { percent }, \\
& \rightarrow{ }^{23} \mathrm{Mg}+n-2.60 \mathrm{MeV}, \quad 2 \text { percent },
\end{aligned}
$$

and

$$
\begin{aligned}
& { }^{12} \mathrm{C}+{ }^{16} \mathrm{O} \rightarrow\left({ }^{28} \mathrm{Si}\right) * \rightarrow{ }^{24} \mathrm{Mg}+\alpha+6.77 \mathrm{MeV}, \quad 40 \text { percent }, \\
& \rightarrow{ }^{27} \mathrm{Al}+p+5.17 \mathrm{MeV}, \quad 50 \text { percent }, \\
& \rightarrow{ }^{27} \mathrm{Si}+n-0.42 \mathrm{MeV}, \quad 10 \text { percent } .
\end{aligned}
$$

The ${ }^{23} \mathrm{Na}$ and ${ }^{23} \mathrm{Mg}$ formed by the proton and neutron branches of carbon burning are converted almost entirely to ${ }^{20} \mathrm{Ne}$ by the reactions ${ }^{23} \mathrm{Mg}(n, p)^{23} \mathrm{Na}$ and ${ }^{23} \mathrm{Na}(p, \alpha){ }^{20} \mathrm{Ne}$. The ${ }^{20} \mathrm{Ne}$ is photodisintegrated to ${ }^{16} \mathrm{O}$. Thus a loop is established which results in the partial conversion of ${ }^{16} \mathrm{O}$ into $\alpha$-particles. This loop may be denoted by the reaction sequence ${ }^{16} \mathrm{O}(\gamma, \alpha){ }^{12} \mathrm{C}\left({ }^{12} \mathrm{C}, \alpha\right)^{20} \mathrm{Ne}(\gamma ; \alpha){ }^{16} \mathrm{O}$ and proceeds at a rate determined by the rate at which ${ }^{16} \mathrm{O}$ is converted to ${ }^{12} \mathrm{C}$ and the fraction of carbon 
which undergoes carbon burning rather than ${ }^{12} \mathrm{C}+{ }^{16} \mathrm{O}$ or photodisintegration. Due to the tendency of the products of the reaction ${ }^{12} \mathrm{C}+{ }^{16} \mathrm{O}$ to combine with free particles of the same sort as the channel that produced them, the net product of the reaction ${ }^{12} \mathrm{C}+{ }^{16} \mathrm{O}$ is ${ }^{28} \mathrm{Si}$ nuclei. Thus, for instance, ${ }^{24} \mathrm{Mg}$ captures $\alpha$-particles via either ${ }^{24} \mathrm{Mg}(\alpha, \gamma){ }^{28} \mathrm{Si}$ or ${ }^{24} \mathrm{Mg}(\alpha, p){ }^{27} \mathrm{Al} ;{ }^{27} \mathrm{Si}$ captures neutrons to form also ${ }^{27} \mathrm{Al}$ via ${ }^{27} \mathrm{Si}(n, p){ }^{27} \mathrm{Al}$; and ${ }^{27} \mathrm{Al}$, for the most part, undergoes $(p, \gamma)$ reactions to form ${ }^{28} \mathrm{Si}$.

\section{c) Equations for $Y\left({ }^{16} \mathrm{O}\right)$ and $Y\left({ }^{12} \mathrm{C}\right)$}

This then is the nuclear scenario, for the lighter species at least, for conditions appropriate to explosive oxygen burning: the ${ }^{16} \mathrm{O}$ is $(1)$ combining with itself to form compound nuclear states of ${ }^{32} \mathrm{~S}$ and at the same time (2) reacting with ${ }^{12} \mathrm{C}$ nuclei produced by its own disintegration with the net production of ${ }^{28} \mathrm{Si}$ and (3) being converted into free $\alpha$-particles by a series of disintegration reactions on the products of carbon burning. The differential equations for the ${ }^{16} \mathrm{O}$ and ${ }^{12} \mathrm{C}$ abundances may then be written approximately as

$$
\begin{aligned}
\frac{d Y\left({ }^{12} \mathrm{C}\right)}{d t} \simeq & -2 Y^{2}\left({ }^{12} \mathrm{C}\right) \lambda_{12}\left({ }^{12} \mathrm{C}\right)-Y\left({ }^{12} \mathrm{C}\right)\left[Y\left({ }^{16} \mathrm{O}\right) \lambda_{12}\left({ }^{16} \mathrm{O}\right)+\lambda_{\gamma 3 \alpha}\left({ }^{12} \mathrm{C}\right)\right] \\
& +Y\left({ }^{16} \mathrm{O}\right)\left[Y_{p} \lambda_{p \alpha}\left({ }^{16} \mathrm{O}\right)+\lambda_{\gamma \alpha}\left({ }^{16} \mathrm{O}\right)\right] \\
\frac{d Y\left({ }^{16} \mathrm{O}\right)}{d t} \simeq & -2 Y^{2}\left({ }^{16} \mathrm{O}\right) \lambda_{16}\left({ }^{16} \mathrm{O}\right)-Y\left({ }^{12} \mathrm{C}\right) Y\left({ }^{16} \mathrm{O}\right) \lambda_{12}\left({ }^{16} \mathrm{O}\right) \\
& -Y\left({ }^{16} \mathrm{O}\right)\left[Y_{p} \lambda_{p \alpha}\left({ }^{16} \mathrm{O}\right)+\lambda_{\gamma \alpha}\left({ }^{16} \mathrm{O}\right)\right]+Y^{2}\left({ }^{12} \mathrm{C}\right) \lambda_{12}\left({ }^{12} \mathrm{C}\right),
\end{aligned}
$$

where $Y_{p}$ is the free proton abundance and the various $\lambda$ 's are given in the Appendix. The ${ }^{12} \mathrm{C}$ abundance quickly achieves a steady state abundance so that its time derivative may be taken equal to zero. Then

$$
\frac{d Y\left({ }^{16} \mathrm{O}\right)}{d t} \simeq-2 Y^{2}\left({ }^{16} \mathrm{O}\right) \lambda_{16}\left({ }^{16} \mathrm{O}\right)-2 Y\left({ }^{16} \mathrm{O}\right) Y\left({ }^{12} \mathrm{C}\right) \lambda_{12}\left({ }^{16} \mathrm{O}\right)-Y^{2}\left({ }^{12} \mathrm{C}\right) \lambda_{12}\left({ }^{12} \mathrm{C}\right),
$$

where

$$
Y\left({ }^{12} \mathrm{C}\right) \simeq-\frac{b+\left(b^{2}-4 a c\right)^{1 / 2}}{2 a}
$$

and

$$
\begin{gathered}
a=2 \lambda_{12}\left({ }^{12} \mathrm{C}\right), \quad b=Y\left({ }^{16} \mathrm{O}\right) \lambda_{12}\left({ }^{16} \mathrm{O}\right)+\lambda_{\gamma 3 \alpha}\left({ }^{12} \mathrm{C}\right), \\
c=-Y\left({ }^{16} \mathrm{O}\right)\left[Y_{p} \lambda_{p \alpha}\left({ }^{16} \mathrm{O}\right)+\lambda_{\gamma \alpha}\left({ }^{16} \mathrm{O}\right)\right] .
\end{gathered}
$$

These equations are valid if two conditions are satisfied: (1) $Y\left({ }^{16} \mathrm{O}\right)\left[Y_{p} \lambda_{p \alpha}\left({ }^{16} \mathrm{O}\right)+\right.$ $\left.\lambda_{\gamma \alpha}\left({ }^{16} \mathrm{O}\right)\right] \gg Y\left({ }^{24} \mathrm{Mg}\right)\left[\lambda_{\gamma p}\left({ }^{24} \mathrm{Mg}\right)+\lambda_{\gamma \alpha}\left({ }^{24} \mathrm{Mg}\right)\right]$, which is true for $T_{9} \leqslant 4.0$ if $Y\left({ }^{16} \mathrm{O}\right) \gg$ $Y\left({ }^{24} \mathrm{Mg}\right)$; and (2) $\lambda_{\gamma \alpha}\left({ }^{20} \mathrm{Ne}\right) \gg Y_{\alpha}\left[\lambda_{\alpha \gamma}\left({ }^{20} \mathrm{Ne}\right)+\lambda_{\alpha p}\left({ }^{20} \mathrm{Ne}\right)\right]$, which is generally true for alpha densities encountered during explosive oxygen burning. In regions of the $\left(\rho, T_{9}\right)$-plane where the destruction of ${ }^{16} \mathrm{O}$ by $(p, \alpha)$ reactions is negligible $\left(T_{9} \leqslant 3.7\right)$ equation (11) gives a closed differential equation for the ${ }^{12} \mathrm{C}$ and ${ }^{16} \mathrm{O}$ abundances as functions of only the variables $Y\left({ }^{16} \mathrm{O}\right), T_{9 i}, \rho_{i}, \chi$, and $t$. Numerical integration of the simple equation will yield good estimates of such interesting quantities as the amount of ${ }^{16} \mathrm{O}$ burned during a given explosion, the amount of alpha production by elements lighter than ${ }^{28} \mathrm{Si}$ during the explosion, and an estimate of the temperature at which strong nuclear activity ceases (the freeze-out temperature). This last quantity is defined for convenience as that temperature below which all further nuclear reactions will 
change the abundance of the primary constituent, ${ }^{16} \mathrm{O}$ in this case, by less than one percent. This choice of one percent was arbitrary. Due to the high temperature dependence of the nuclear reactions involved, the exact value is not critical. Hence the freeze-out temperature $T_{9 f}$ is mathematically defined by

$$
\int_{0}^{T_{9 f}}\left[\frac{d Y\left({ }^{16} \mathrm{O}\right)}{d t}\right]\left(\frac{d t}{d T_{9}}\right) d T_{9} \equiv 0.01 Y\left({ }^{16} \mathrm{O}\right),
$$

This equation can be evaluated using equations (2), (3), and (11) to yield $T_{9 f}$ for a given set of initial conditions. Since ${ }^{16} \mathrm{O}$ is the primary source of free particles for oxygen burning, the "freezing out" of its abundance implies that the abundances of all important species at $T_{9 f}$ should be a fair representation of their final values, i.e., their values when $T_{9}$ goes to zero.

\section{d) The Role of ${ }^{24} \mathrm{Mg}$}

Thus far we have considered only interactions of species lighter than magnesium. We have seen that the nuclear combustion of ${ }^{16} \mathrm{O}$ yields a fairly complicated network of reactions the net result of which is the production of species having $A \geq 24$ and the production of free $\alpha$-particles which are then captured by species of $A \geq 24$. In the range of explosive-oxygen-burning temperatures ${ }^{24} \mathrm{Mg}$ has by far the largest crosssection for $(\alpha, \gamma)$ and $(\alpha, p)$ reactions of any species that accumulates a nontrivial abundance. Moreover, due to the very great alpha separation energy of ${ }^{28} \mathrm{Si}$ an $\alpha-$ particle captured by ${ }^{24} \mathrm{Mg}$ tends to remain bound. Thus most of the $\alpha$-particles liberated by the disintegration loop centered about ${ }^{16} \mathrm{O}$, as well as the alpha branches of the reactions ${ }^{16} \mathrm{O}+{ }^{16} \mathrm{O}$ and ${ }^{12} \mathrm{C}+{ }^{16} \mathrm{O}$, will add onto ${ }^{24} \mathrm{Mg}$ as long as any significant abundance of ${ }^{24} \mathrm{Mg}$ remains. The decreased alpha density due to the high alpha cross-section of ${ }^{24} \mathrm{Mg}$ causes ${ }^{31} \mathrm{P}(p, \alpha){ }^{28} \mathrm{Si}$ to occur at a larger rate than ${ }^{28} \mathrm{Si}(\alpha, p){ }^{31} \mathrm{P}$, so that ${ }^{28} \mathrm{Si}$ is also the product of the proton channel of the reaction ${ }^{16} \mathrm{O}+{ }^{16} \mathrm{O}$. As mentioned previously, the net result of the reaction ${ }^{12} \mathrm{C}+{ }^{16} \mathrm{O}$ is always ${ }^{28} \mathrm{Si}$. Thus, as is illustrated in figure $3 a$, the almost exclusive product of explosive oxygen burning is ${ }^{28} \mathrm{Si}$ as long as ${ }^{24} \mathrm{Mg}$ remains undepleted.

Once ${ }^{24} \mathrm{Mg}$ has been depleted figure $3 b$ shows that the net nuclear flows above $A=24$ change a great deal. Now the primary seed for alpha-consuming reactions is ${ }^{28} \mathrm{Si}$ and, to some extent, ${ }^{32} \mathrm{~S}$, but other reactions, mainly involving $\alpha$-particles liberated by the ${ }^{16} \mathrm{O}$ disintegration chain and the reaction ${ }^{16} \mathrm{O}+{ }^{16} \mathrm{O}$, are tending to produce species of $A \geq 32$. Furthermore, the free-alpha density has risen to the point where ${ }^{28} \mathrm{Si}(\alpha, p){ }^{31} \mathrm{P}$ is no longer dominated by the reverse flow. Instead ${ }^{31} \mathrm{P}$ is destroyed by $(p, \gamma)$ reactions so that the main result of ${ }^{16} \mathrm{O}+{ }^{16} \mathrm{O}$ is now ${ }^{32} \mathrm{~S}$. As a result the abundance of species having $A \geq 32$ begins to rise dramatically. The evolution of major abundances as time passes is shown in figure 5 and displays the key features of this discussion.

\section{e) Location of Excess Neutrons}

It is also interesting to trace the history of the neutron excess through the evolution. At the very beginning of the burning, ${ }^{26} \mathrm{Mg}$ is converted into neutron-rich silicon isotopes by ${ }^{26} \mathrm{Mg}(\alpha, n){ }^{29} \mathrm{Si}$ and ${ }^{29} \mathrm{Si}(n, \gamma){ }^{30} \mathrm{Si}$. During this time a high value of the freeneutron density $\left(Y_{n} \sim 10^{-8}\right)$ prevails. After ${ }^{26} \mathrm{Mg}$ is depleted the free-neutron density drops about an order of magnitude and continues to decrease as the evolution progresses. Following the depletion of ${ }^{24} \mathrm{Mg}$ the neutron-rich silicon isotopes are converted to neutron-rich sulfur isotopes by various reaction chains, typical of which are ${ }^{30} \mathrm{Si}(\alpha, p){ }^{31} \mathrm{P}(p, \gamma){ }^{32} \mathrm{~S}(\alpha, p){ }^{35} \mathrm{Cl}(\gamma, p){ }^{34} \mathrm{~S}$ and ${ }^{28} \mathrm{Si}(\alpha, \gamma){ }^{32} \mathrm{~S}(n, \gamma){ }^{33} \mathrm{~S}$. Still later a part of the sulfur isotopes are converted to argon isotopes chiefly by the reactions 


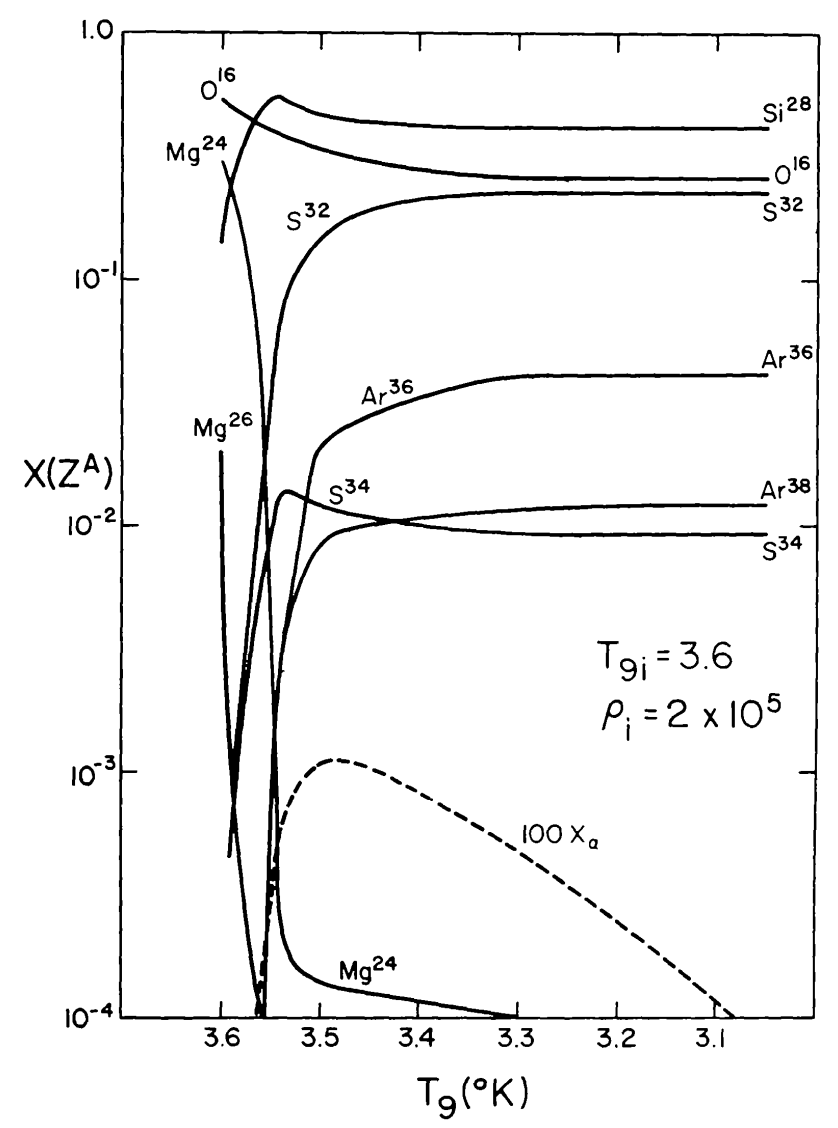

Fig. 5.-The mass fractions of several important species are shown as a function of the decreasing temperature during the "standard" explosive-oxygen-burning run. Also shown is the value of the alpha mass fraction which has been multiplied by a factor of 100 for comparison purposes.

${ }^{34} \mathrm{~S}(p, \gamma){ }^{35} \mathrm{Cl}(p, \gamma){ }^{36} \mathrm{Ar},{ }^{32} \mathrm{~S}(\alpha, p){ }^{35} \mathrm{Cl}(\alpha, p){ }^{38} \mathrm{Ar}$ and ${ }^{33} \mathrm{~S}(\alpha, n){ }^{36} \mathrm{Ar}(n, \gamma){ }^{37} \mathrm{Ar}$. Finally at a temperature of about $T_{9}=3.2$, the strong interactions have slowed to the extent that no further transformation of the matter occurs. The excess neutrons are left bound, chiefly in argon and sulfur isotopes.

\section{f) Free-Particle Exposures}

The possibility of interactions involving seed nuclei that would be present in a Population I star and the free-particle abundances during explosive oxygen burning will not be considered in this paper. A recent investigation of seed reactions during explosive carbon burning by Howard et al. (1972) has shown that a number of relatively rare neutron-rich species in the mass range $36 \leq A \leq 76$ may be synthesized in amounts that compare well with their solar abundances. At the higher temperatures appropriate to explosive oxygen burning however, we would not expect the production of such neutron-rich species. Even if such species were produced during the early neutron-dominated phase of explosive oxygen burning (before ${ }^{26} \mathrm{Mg}$ is depleted), they would be quickly destroyed during the later phases of the explosion by photoneutron reactions or reactions with protons and $\alpha$-particles. Thus we do not expect anything resembling an $r$-process to take place under explosive-oxygen-burning conditions. However, the reaction of species heavier than iron with free particles and the photon bath may be important. Preliminary calculations show that a complex system of reactions involving seed nuclei under conditions similar to explosive oxygen burning may lead to results that resemble a $p$-process. A further investigation is underway. 
EXPLOSIVE BURNING OF OXYGEN AND SILICON

TABLE 1

Particle EXPosure Strengths for Explosive OXYGen Burning

\begin{tabular}{lllllll}
\hline & \multicolumn{7}{c}{$T_{9 i}$} \\
\cline { 2 - 7 } & 3.4 & 3.6 & 3.8 & 3.3 & 3.7 & 3.9 \\
\hline$\rho_{i} \ldots \ldots \ldots \ldots$ & $1.0(+4)$ & $2.0(+5)$ & $1.5(+6)$ & $1.0(+4)$ & $1.5(+6)$ & $1.2(+7)$ \\
$X_{i} \ldots \ldots \ldots \cdots$ & 0.54 & 0.54 & 0.54 & 0.70 & 0.70 & 0.70 \\
$S_{n} \ldots \ldots \ldots \cdots$ & $4.6(-5)$ & $5.3(-5)$ & $5.9(-5)$ & $3.2(-5)$ & $3.3(-5)$ & $2.9(-5)$ \\
$S_{p} \ldots \ldots \ldots$ & $6.2(-1)$ & $8.3(-1)$ & $2.5(0)$ & $9.3(-2)$ & $9.3(-1)$ & $2.0(0)$ \\
$S_{\alpha} \ldots \ldots \ldots$ & $3.5(0)$ & $3.3(0)$ & $5.6(0)$ & $8.0(-1)$ & $3.0(0)$ & $4.0(0)$ \\
\hline
\end{tabular}

Representative values of the free-particle source strengths during explosive oxygen burning are given in table 1 . The table gives values of the quantity

$$
S_{k} \equiv \int_{0}^{\infty} Y_{k} \rho d t
$$

for neutrons, protons, and $\alpha$-particles for a variety of runs. The initial composition in each case is specified by the initial ${ }^{16} \mathrm{O}$ mass fraction. The remainder of the initial mass is two percent ${ }^{26} \mathrm{Mg}$ by mass fraction and ${ }^{24} \mathrm{Mg}$ and ${ }^{28} \mathrm{Si}$ in the ratio of $2: 1$ by mass. The largest contribution to $S_{k}$ always occurs fairly early in the run before the temperature and density have dropped significantly, so that the initial values of $T_{9}$ and $\rho$ may be employed to estimate seed-reaction rates. The reader should be forewarned that simple consideration of the forward flow on a given seed reaction is insufficient since reverse flows, in particular, photodisintegration, are quite important at these temperatures. Nevertheless, the values given for $S_{k}$ are useful for estimating whether a given seed reaction may or may not be possible in the given time.

\section{QUASI-EQUILIBRIUM DURING EXPLOSIVE OXYGEN BURNING}

As TA70 pointed out, explosive oxygen burning has a quasi-equilibrium character, at least for nuclei between ${ }^{28} \mathrm{Si}$ and the $\mathrm{Ca}$ isotopes. We will consider how this comes about. An examination of the nuclear flows above $A=28$ during the early stages of explosive oxygen burning reveals the existence of blocks of elements whose abundances are approaching steady state with respect to the externally fixed free-particle densities. Stated in terms of equation (5) this means that there exist groups of species which may be interconnected by links of the form $\lambda_{j k}(i)$ for which the net material flow, $F_{j k: i}$, between the two species $i$ and $l=i+j-k$ is found to be much smaller in absolute magnitude than the forward flow, $f_{j k: i}$, from the species $i$ to the species $l$. That is

$$
F_{j k: i} \equiv\left|f_{j k: i}-f_{k j: l}\right| \ll f_{j k: i},
$$

where the inequality applies during quasi-equilibrium and

$$
f_{j k: i} \equiv Y(i) Y(j) \lambda_{j k}(i) .
$$

During the very early stages of the explosion, these blocks or "cluster-equilibrium groups" as they may be called are comprised of only several nuclear species. But as the evolution progresses, many of these small cluster-equilibrium groups are often found to merge into larger groups. One of these groups, eventually comprised of all the species in the $28 \leq A \leq 45$ mass range, contains almost all of the mass (exclusive of ${ }^{16} \mathrm{O}$ itself) and most of the species produced in the explosive calculation of the 
previous section. The most abundant nucleus throughout this merging process is ${ }^{28} \mathrm{Si}$, because the early burning produces primarily ${ }^{28} \mathrm{Si}$ and because the unusually tight binding of ${ }^{28} \mathrm{Si}$ renders it almost stable against photodisintegration.

A similar type of behavior was discovered by BCF to occur during incomplete silicon burning. They find that after an initial period of adjustment a cluster-equilibrium group, or as they call it a "quasi-equilibrium distribution" comprised of species in the mass range $28 \leq A \leq 62$ develops. Choosing ${ }^{28} \mathrm{Si}$ as the "base element" because of its physical dominance, they showed that in a quasi-equilibrium group the abundance of any member is known as a simple function of the free-particle densities ( $\alpha$-particles, neutrons, and protons) and the ${ }^{28} \mathrm{Si}$ abundance at the given temperature and density. Using $N(i)=\rho N_{\mathrm{A}} Y(i)$ for the number density of species $i$ their equation (3) becomes

$$
Y_{\mathrm{qe}}(i)=C_{i}\left(T_{9}\right) Y\left({ }^{28} \mathrm{Si}\right)\left[\rho N_{\mathrm{A}} Y_{\alpha}\right]^{\delta \alpha^{\prime}}\left[\rho N_{\mathrm{A}} Y_{p}\right]^{\delta p^{\prime}}\left[\rho N_{\mathrm{A}} Y_{n}\right]^{\delta n^{\prime}},
$$

where $C_{i}\left(T_{9}\right)$ is a function of the species $i$ and the temperature tabulated by BCF and $\delta_{\alpha}{ }^{\prime}, \delta_{p}{ }^{\prime}$, and $\delta_{n}{ }^{\prime}$ (also tabulated by BCF) specify the number of $\alpha$-particles and nucleons in the species $i$ in excess of the number in ${ }^{28} \mathrm{Si}$. Generalizing their arguments it follows that in any cluster-equilibrium group specifying the free-particle abundances and any one key abundance in the group (i.e., the base element) at a given temperature uniquely determines the abundances of all other species in the group.

If photodisintegration reactions and forward reactions involving $\alpha$-particles are sufficiently operative that the free-alpha abundance is in equilibrium with the neutron and proton abundances, then the additional equation

$$
Y_{\mathrm{q} \theta}(\alpha)=C_{\alpha}\left(T_{9}\right)\left(\rho N_{\mathrm{A}}\right)^{3} Y_{n}{ }^{2} Y_{p}{ }^{2}
$$

may be used to eliminate one of the free-particle abundances as an independent variable in equation (15). This approximation is usually valid during explosive oxygen burning as well as in silicon burning. In both cases equilibrium between $\alpha$-particles and the free protons and neutrons is maintained by rapid reaction chains like ${ }^{28} \mathrm{Si}(\alpha, \gamma){ }^{32} \mathrm{~S}(\gamma, p)^{31} \mathrm{P}(\gamma, p)^{30} \mathrm{Si}(\gamma, n)^{29} \mathrm{Si}(\gamma, n)^{28} \mathrm{Si}$. If the bulk of the mass is contained in one cluster-equilibrium group, such as the case of the silicon quasi-equilibrium, then the simultaneous validity of equation (16) means that the abundances of species in that group can be determined by $\rho, T_{9}, \eta$, and some base abundance. In that case the necessity of solving the large matrix of coupled nuclear flows is eliminated. Only a few nonequilibrium flows must be considered, resulting in a great reduction in the amount of work required to follow a given nuclear evolution.

\section{a) Quasi-Equilibrium Clusters}

Such considerations motivate us to calculate the silicon-based quasi-equilibrium distribution during each run of the explosive-oxygen-burning network. At each printout the computer writes out the logarithmic ratio $r_{j}$ of the abundance of each species calculated, assuming a silicon-based quasi-equilibrium distribution for all species, to the actual abundance obtained by network evolution. From that set of numbers it is a simple matter to determine how well the quasi-equilibrium distribution approximates the actual abundance distribution throughout the evolution. Table 2 lists the values of $r_{j}$ for the nuclei in the explosive-oxygen-burning network for the standard oxygen-burning run at an elapsed time of $0.090 \mathrm{~s}$. This table illustrates the striking tendency of elements to segregate into cluster-equilibrium groups. Those elements which have $r_{j} \simeq 0$ are in quasi-equilibrium with ${ }^{28} \mathrm{Si}$. Species characterized by similar values of $r_{j}$ but having $r_{j} \neq 0$ are in mutual equilibrium with one another but not with ${ }^{28} \mathrm{Si}$. This separateness is due to the existence of reaction links which have not yet come into equilibrium. This occurs, for instance, in the vicinity of the relatively 
TABLE 2

VAlues of $r_{\boldsymbol{f}}$ for Selected Species during Explosive Oxygen BuRning*

\begin{tabular}{|c|c|c|c|c|c|}
\hline Species & $r_{j}$ & Species & $r_{j}$ & Species & $r_{j}$ \\
\hline & +0.03 & ${ }^{37} \mathrm{Cl}$ & 0.06 & ${ }^{46} \mathrm{Ti}$ & 1.84 \\
\hline${ }^{12} \mathrm{C} \ldots$ & -1.64 & ${ }^{36} \mathrm{Ar} \ldots \ldots \ldots$ & 0.02 & ${ }^{47} \mathrm{Ti}$ & 2.23 \\
\hline$\ldots \ldots$ & -3.42 & ${ }^{37} \mathrm{Ar} \quad \ldots \ldots \ldots$ & 0.03 & ${ }^{48} \mathrm{Ti}$ & 3.43 \\
\hline$\ldots \ldots$ & -1.64 & ${ }^{38} \mathrm{Ar} \ldots \ldots \ldots$ & 0.06 & ${ }^{47} \mathrm{~V}$ & 1.84 \\
\hline${ }^{16} \mathrm{O} \ldots \ldots$ & -3.63 & ${ }^{39} \mathrm{Ar} \ldots \ldots \ldots$ & 0.06 & ${ }^{48} \mathrm{~V}$ & 2.29 \\
\hline${ }^{20} \mathrm{Ne} \ldots \ldots \ldots$ & -3.64 & ${ }^{40} \mathrm{Ar} \ldots \ldots \ldots$ & 0.06 & ${ }^{49} \mathrm{~V}$ & 3.45 \\
\hline${ }^{23} \mathrm{Na} \ldots \ldots \ldots$ & -3.70 & ${ }^{39} \mathrm{~K} \ldots$ & 0.06 & ${ }^{50} \mathrm{~V}$ & 3.62 \\
\hline${ }^{24} \mathrm{Mg} \ldots \ldots$ & -0.93 & ${ }^{40} \mathrm{~K} \ldots \ldots$ & 0.07 & ${ }^{48} \mathrm{Cr}$ & 1.87 \\
\hline${ }^{25} \mathrm{Mg} \ldots \ldots \ldots$ & -0.92 & ${ }^{41} \mathrm{~K} \ldots \ldots \ldots$ & 0.06 & ${ }^{49} \mathrm{Cr}$ & 2.76 \\
\hline${ }^{26} \mathrm{Mg} \ldots \ldots \ldots$ & -0.96 & ${ }^{40} \mathrm{Ca} . . . .$. & 0.05 & ${ }^{50} \mathrm{Cr}$ & 3.58 \\
\hline${ }^{27} \mathrm{Al} \ldots \ldots \ldots$ & -0.91 & ${ }^{41} \mathrm{Ca} . . . \ldots \ldots$ & 0.05 & ${ }^{51} \mathrm{Cr}$ & 3.68 \\
\hline${ }^{28} \mathrm{Si} \quad \ldots \ldots \ldots$ & 0 & ${ }^{42} \mathrm{Ca} \ldots . .$. & 0.07 & ${ }^{51} \mathrm{Mn} .$. & 3.58 \\
\hline${ }^{29} \mathrm{Si}$ & +0.01 & ${ }^{43} \mathrm{Ca} . \ldots \ldots \ldots$ & 0.09 & ${ }^{52} \mathrm{Mn} \quad \ldots$ & 3.69 \\
\hline${ }^{30} \mathrm{Si}$ & +0.01 & ${ }^{44} \mathrm{Ca} \ldots \ldots \ldots \ldots$ & 1.11 & ${ }^{53} \mathrm{Mn} \quad \ldots$ & 4.66 \\
\hline${ }^{30} \mathbf{P}$ & +0.01 & ${ }^{45} \mathrm{Ca} \ldots \ldots \ldots \ldots$ & 1.13 & ${ }^{52} \mathrm{Fe} \ldots$ & 3.55 \\
\hline${ }^{31} \mathbf{P}$ & +0.02 & ${ }^{46} \mathrm{Ca} . \ldots \ldots \ldots$ & 2.06 & ${ }^{53} \mathrm{Fe}$ & 3.98 \\
\hline${ }^{32} \mathbf{P}$ & +0.04 & ${ }^{47} \mathrm{Ca} . \ldots \ldots \ldots$ & 2.20 & ${ }^{54} \mathrm{Fe}$ & 4.64 \\
\hline${ }^{33} \mathbf{P}$ & +0.05 & ${ }^{48} \mathrm{Ca} \ldots \ldots \ldots$ & 2.20 & ${ }^{55} \mathrm{Fe}$ & 4.65 \\
\hline${ }^{31} \mathrm{~S} . .$. & +0.01 & ${ }^{43} \mathrm{Sc} \ldots \ldots \ldots \ldots$ & 0.07 & ${ }^{56} \mathrm{Fe}$ & 4.70 \\
\hline${ }^{32} \mathrm{~S} \ldots$ & +0.01 & ${ }^{44} \mathrm{Sc} \ldots \ldots \ldots \ldots$ & 0.10 & ${ }^{55} \mathrm{Co} \ldots \ldots \ldots$ & 4.64 \\
\hline (33. & +0.02 & ${ }^{45} \mathrm{Sc} \ldots \ldots \ldots \ldots$ & 1.14 & ${ }^{56} \mathrm{Co} \ldots \ldots \ldots$ & 4.65 \\
\hline${ }^{34} \mathrm{~S} \ldots$. & +0.03 & ${ }^{46} \mathrm{Sc} \ldots \ldots \ldots \ldots$ & 1.62 & ${ }^{57} \mathrm{Co} \ldots \ldots \ldots$ & 4.70 \\
\hline & +0.04 & ${ }^{47} \mathrm{Sc} \ldots \ldots \ldots \ldots$ & 2.27 & ${ }^{56} \mathrm{Ni} \quad \ldots \ldots \ldots$ & 4.61 \\
\hline${ }^{36} \mathrm{~S} .$. & +0.05 & ${ }^{44} \mathrm{Ti} \ldots \ldots \ldots \ldots$ & 0.05 & ${ }^{57} \mathrm{Ni}$ & 4.63 \\
\hline${ }^{35} \mathrm{Cl}$. & +0.05 & ${ }^{45} \mathrm{Ti} \ldots \ldots \ldots \ldots$ & 0.76 & ${ }^{58} \mathrm{Ni} \ldots \ldots \ldots$ & 4.67 \\
\hline${ }^{36} \mathrm{Cl}$. & +0.05 & & & & \\
\hline
\end{tabular}

* $T_{9}=3.49, t=0.090 \mathrm{~s}$.

weakly bound titanium and scandium isotopes, whose low abundances inhibit the flow of material from the $A \leqslant 45$ region into the $A>45$ region. The little that does "break through" distributes itself in such a manner as to maintain the clusterequilibrium form of the next group, which feeds the next cluster, and so on. However, such leaks are generally not sufficient to bring all the various clusters into mutual equilibrium during the time available, under conditions appropriate to explosive oxygen burning.

In order to determine when quasi-equilibrium is a good approximation and which members comprise the various clusters under different conditions, it will be useful to examine the approach to quasi-equilibrium. The following semiquantitative considerations will help in understanding our results. Consider two nuclei, $Y(1)$ and $Y(2)$, both heavier than ${ }^{28} \mathrm{Si}$, which may be coupled by the reaction link $\lambda_{j k}(1)$ whose inverse is $\lambda_{k j}(2)$. Both $Y(1)$ and $Y(2)$ may be subject to many other reactions; but this link should be representative. Let us make the identification that $Y(1)$ is the lighter of the pair under consideration and that initially the ratio $Y(1) / Y(2)$ greatly exceeds its quasi-equilibrium value. Now in explosive oxygen burning the forward flows (flows that increase $A$ or $Z$ ) are predominating in the creation of new species, particularly during the early stages of the evolution when equilibration is taking place. The forward flow from 1 to 2 due to the characteristic reaction $(j k)$ is $f_{j k ; 1}=Y(1) Y(j) \lambda_{j k}(1)$. The abundance of species 1 is most likely maintained or even increased [i.e., $d Y(1) / d t \geq 0$ ] by other flows from lighter species during the time equilibration is occurring. Therefore it is realistic to assume that the value of $f_{j k ; 1}$ does not decrease over the time interval of interest. As species 2 begins to approach its equilibrium value, the reverse 
flow $f_{k j: 2}$ becomes very nearly equal to $f_{j k: 1}$ and $Y(2)$ assumes a stationary value

$$
Y_{\mathrm{qe}}(2)=Y(1) \frac{Y(j) \lambda_{j k}(1)}{Y(k) \lambda_{k j}(2)} .
$$

A limit to the time that it takes $Y(2)$ to approach this value can be estimated by the requirement that during a given time interval $\Delta t$ the average value of $f_{j k: 1}$ satisfies

$$
\beta f_{j k: 1} \Delta t \geq Y_{\mathrm{qe}}(2),
$$

where $\beta$ is the fraction of the flow into $Y(2)$ that remains in $Y(2)$ rather than passing on to species other than species 1 by means of other reactions. If $f_{j k: 1}$ is the chief flow leading to the creation of $Y(2)$, then the above restriction is an equality and equilibrium between $Y(1)$ and $Y(2)$ should be approximately attained in a time, $\tau_{12}$, such that

$$
\beta Y(1) Y(j) \lambda_{j k}(1) \tau_{12}=Y(1) \frac{Y(j) \lambda_{j k}(1)}{Y(k) \lambda_{k j}(2)},
$$

or

$$
\tau_{12}=\left[\beta Y(k) \lambda_{k j}(2)\right]^{-1} .
$$

If $f_{j k: 1}$ is not the chief flow leading to the creation of $Y(2)$, then the value of $\tau_{12}$ should represent an upper bound to the time for the equilibration. The value of $\beta$ that should be employed in equation (18) will vary from species to species and link to link, but it is usually not very small compared to unity for most species during the time equilibration is taking place. A notable exception occurs when the species denoted $Y(2)$ is in a steady state with respect to a strong flow passing through it. (Note the distinction between quasi-equilibrium and steady state: the former is used to denote a link for which $F_{j k: i} \ll f_{j k: i}$; the latter denotes $\sum_{j k} f_{j k: i} \simeq \sum_{l m x} f_{l m: x}$, where the first sum is the flow out of species $i$ and the second sum, subject to the particle constraint $i=x+l-m$, is the flow into species $i$.) Then a small value for $\beta$ is obtained and $\tau_{12}$ becomes larger. Such cases are exceptions, however, and generally it appears likely that equilibration between two species will occur on a time scale on the order of the lifetime of the heavier species against the reverse reaction linking the pair. Most of the important reverse flows in explosive oxygen burning are due to photodisintegration reactions. Hence the approximate time scale given in equation (18) may be written in the simple form

$$
\tau_{12}=\left[\beta \lambda_{y j}(2)\right]^{-1},
$$

where $\lambda_{y j}(2)$ is a photodisintegration reaction linking the heavier species 2 to species 1 . The time for linking a group of species should be on the order of the slowest photodisintegration rate required for the interconnecting of the various species. How well does this rule of thumb work?

In figure $6 a$ are shown clusters of elements which may be interconnected by photodisintegration rates such that $\lambda_{\gamma j}>1 / t$ evaluated at $t=2.13 \times 10^{-3} \mathrm{~s}$ into the standard run, when $T_{9}=3.596$. The logarithmic ratio $r_{j}$ at that time is also given. The fact that the groups segregated by the photodisintegration rates generally correspond to clusters having about the same value of $r_{j}$ substantiates the contention that equilibrium between species is achieved on a time scale approximately given by the lifetime of the heavier species against photodisintegration to the lighter one. This is a very useful result. Since the photodisintegration rates can be determined from the formulae in the Appendix without prior knowledge of the free-particle densities, it is usually possible to estimate the equilibrium character of a distribution at a given temperature and elapsed time without further recourse to the computer. 
It is also interesting to note that in figure $6 a$ each alpha nucleus, with the exception of ${ }^{44} \mathrm{Ti}$, is a member of a separate cluster. This is most likely a binding-energy effect. Photodisintegration rates depend in a very critical fashion upon the separation energy. Figure $6 b$ illustrates this behavior. In the figure are shown groupings, again similar to the cluster grouping obtained by examination of the $r_{j}$ values, only this time the cluster boundaries have been determined by the restriction that the $Q$-value for the forward reaction between two species involving a photon in the exit channel be less than some empirical amount. The empirical number decreases with the charge of the particle in the entrance channel, due to the energy lost in penetrating the Coulomb barrier. At a later time in the evolution, a larger value of $Q$ for the various reactions might be "allowed" and a more consolidated grouping would result. This " $Q$ criteria" is important to remember when designing a reaction network in a new region of the periodic chart. The boundary of the network should be taken to coincide as closely as possible with the natural boundary given by reaction links that require very large $Q$-values.

In figure $6 c$ the cluster groupings as determined by photodisintegration rates is shown at a later time in the standard run. Many of the smaller clusters seen previously have now blended into larger clusters so that quasi-equilibrium now prevails in the silicon-to-calcium region. This result is important since almost all the mass, exclusive of ${ }^{16} \mathrm{O}$ itself, is contained in this region. Again the clustering predicted by photodisintegration rates is confirmed by the grouping of the $r_{j}$ values. A similar grouping might also be obtained if one alternatively chose the criteria $Q(n, \gamma) \leqslant 11.0, Q(p, \gamma) \lesssim$ 9.5 , and $Q(\alpha, \gamma) \leqslant 6.9$. This grouping represents the maximum consolidation to be achieved during this run. Further merging would have required a higher temperature or longer time scale so that sufficient material could break through the $A=45$ "barrier" to bring the iron-peak elements into quasi-equilibrium with ${ }^{28} \mathrm{Si}$. This phenomena will be discussed at greater length later when the merging of explosive oxygen burning into silicon burning is considered in $\S \mathrm{VI} a$.

\section{b) Freeze-out Corrections}

After attaining quasi-equilibrium in the silicon-to-calcium region the abundance distribution continues to evolve as more material in the form of ${ }^{28} \mathrm{Si}$ nuclei and $\alpha-$ particles is added on by oxygen burning. As the temperature drops below the point necessary for sustaining strong nuclear flows the large groups tend to refragment back into the smaller clusters from which they were formed. The group distribution at $T_{9}=2.56$ looks very similar to that shown in figure $6 a$. Finally, the abundances of the individual species freeze out and the distribution undergoes no further nuclear processing by strong interactions.

Although the silicon-to-calcium quasi-equilibrium, once attained, is maintained until quite late in the evolution $\left(T_{9} \sim 3.0\right)$ the abundances of most important species change very little once the ${ }^{16} \mathrm{O}$ abundance freezes out. Once oxygen burning per se ceases to maintain the necessary free-particle densities, substantial nuclear composition changes can only occur by the photodisintegration-rearrangement of species present. In any explosive-oxygen-burning run which creates mainly species in the $28 \leq A \leq 45$ region, the temperature is rapidly becoming too low for such reactions to matter except possibly for species with very low mass fractions. Thus the silicon-to-calcium quasi-equilibrium distribution calculated at $T_{9 f}$ should be a good approximation to the final abundances of species in that group. Table 3 shows to what extent the final abundances are represented by the quasi-equilibrium distribution calculated at $T_{9 f}$ for a variety of initial conditions. It seems that, not only is the agreement remarkable (better than a factor of 2 for even low abundance species), but the errors for each species all appear to be in the same direction and of about the same magnitude for all 
(a)

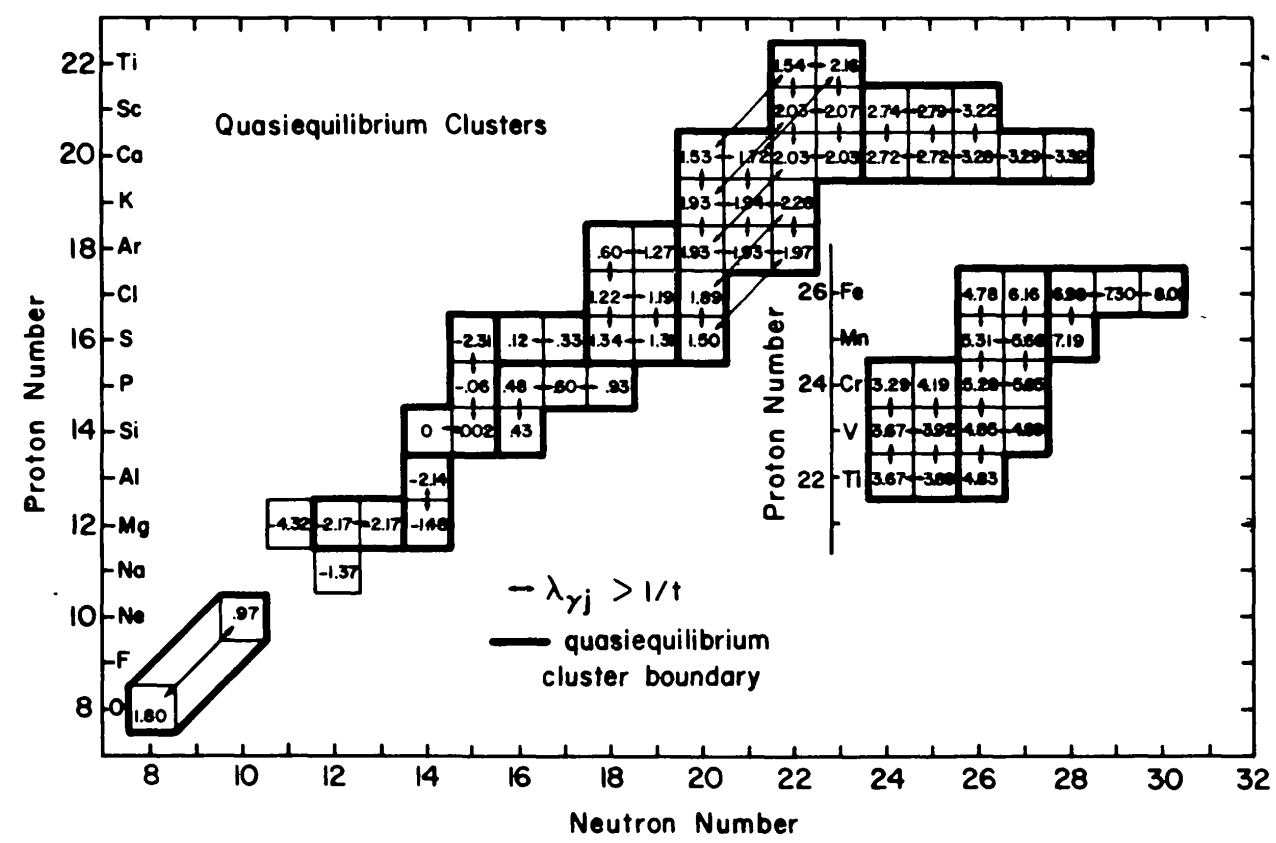

(b)

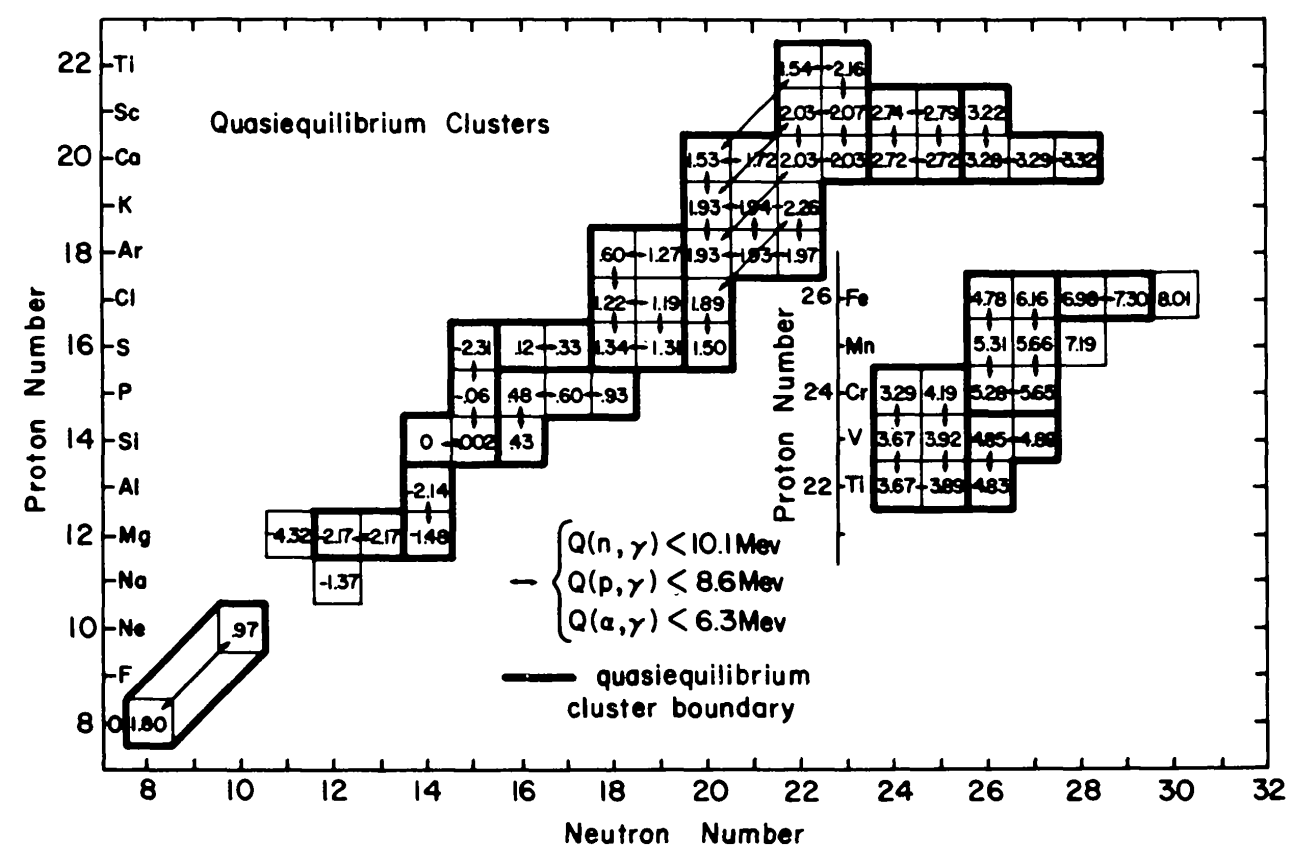

FIGs. $6 a$ and $6 b$ 
(c)

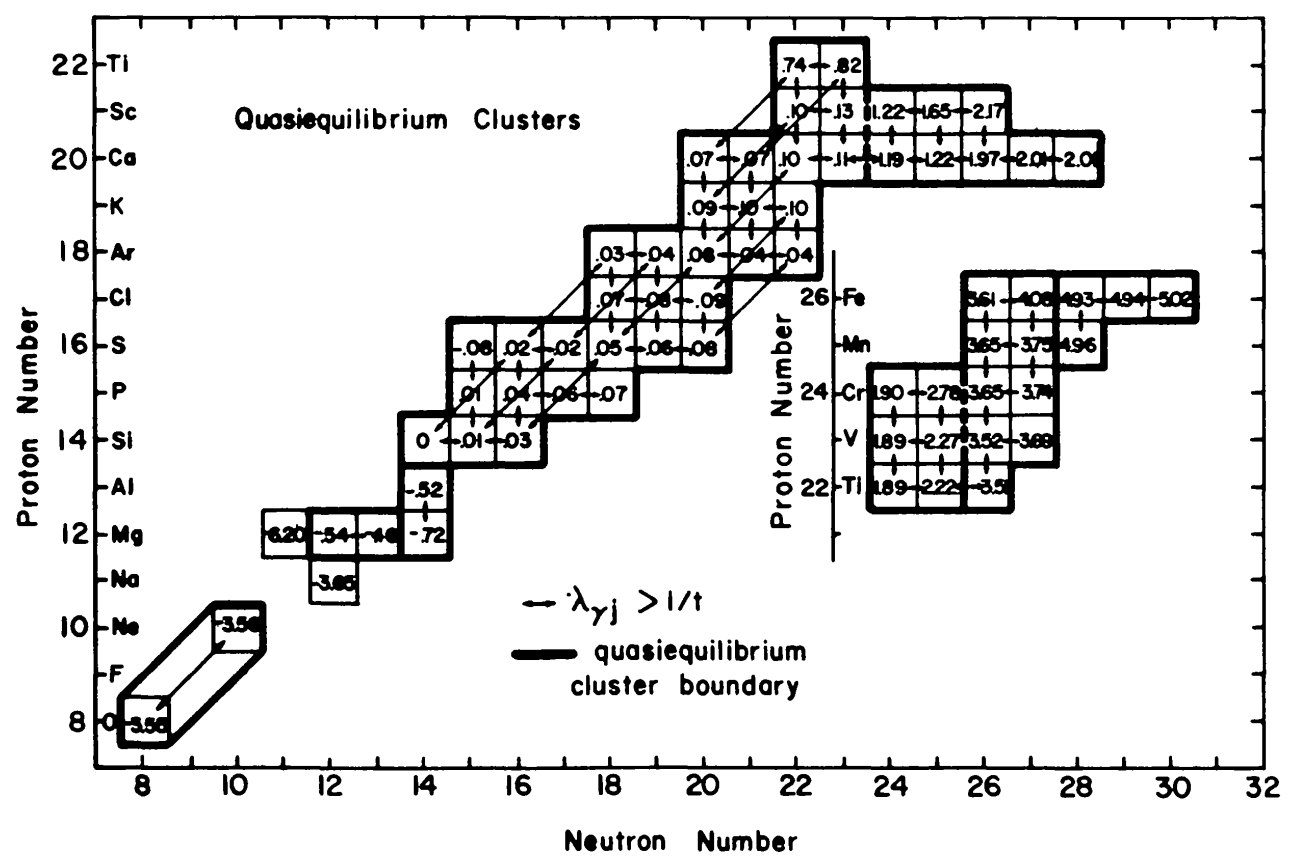

FIGS. $6 a, b, c$.-Quasi-equilibrium clusters as determined by photodisintegration rates early in explosive oxygen burning. Values of the quantity $r_{j}$ are given for each species at a times $t_{a}=t_{b}=$ $2.13 \times 10^{-3}$ s and $t_{c}=7.55 \times 10^{-2}$ s into the evolution of the "standard" explosive-burning run. The temperatures at these times are $T_{9 a b}=3.596$ and $T_{9 c}=3.459$. Also shown are: $(a)$ photodisintegration links that are characterized by rates which at this temperature are larger than the reciprocal of the elapsed time (i.e., $\lambda_{y j}>470$ ). Dark boundaries enclose groups of elements which may be interconnected by such links; $(b)$ photodisintegration links that are characterized by $Q$-values for the forward reaction that are less than the empirical number shown in the figure. The delineation of clusters is very similar to that shown in fig. $6 a ;(c)$ photodisintegration links that are characterized by rates which at this temperature are larger than the reciprocal of the elapsed time (i.e., $\lambda_{y j}>13$ ). Dark boundaries enclose groups of elements which may be interconnected by such links. The boundaries in the vicinity of $N=24$ and $N=26$ are shown as dotted lines due to anomalously small values of $\beta$ for the reaction links ${ }^{43} \mathrm{Ca}(n, \gamma)^{44} \mathrm{Ca}$ and ${ }^{47} \mathrm{Ti}(n, \gamma)^{48} \mathrm{Ti}$.

runs considered. Thus a fairly reliable "freeze-out correction" (denoted $\delta_{f} \pm \sigma$ in table 3) may be computed. Since for all species listed in table 3 the value of $\sigma$ is less than 0.05 , the final abundance of each of these species should be given to within about 15 percent by

$$
X_{f}(i)=\left[X_{\mathrm{qe}}(i)\right]_{T_{9 f}} \times 10^{\delta_{f}^{(i)}}
$$

Generally, the error introduced by this approximation will be less than the error inherent in the experimental determination of the solar-system abundance of the species.

This result is very important. At a given $\eta$, freeze-out temperature, and freeze-out density, it specifies that any two nuclear evolutions which produce the same alpha mass fraction $X_{\alpha}$ are equivalent so far as the nucleosynthesis of abundant species is concerned. The entire previous history of the material is contained in these four parameters. Thus any simple network which correctly tracks the alpha density, such as the simple network described in $\S \mathrm{Vc}$, is sufficient to determine to a fair degree of accuracy the abundances resulting from an explosive-oxygen-burning run which achieves silicon-to-calcium quasi-equilibrium. 
TABLE 3

CHANGES IN ABUNDANCE DURING FREEZE-OUT

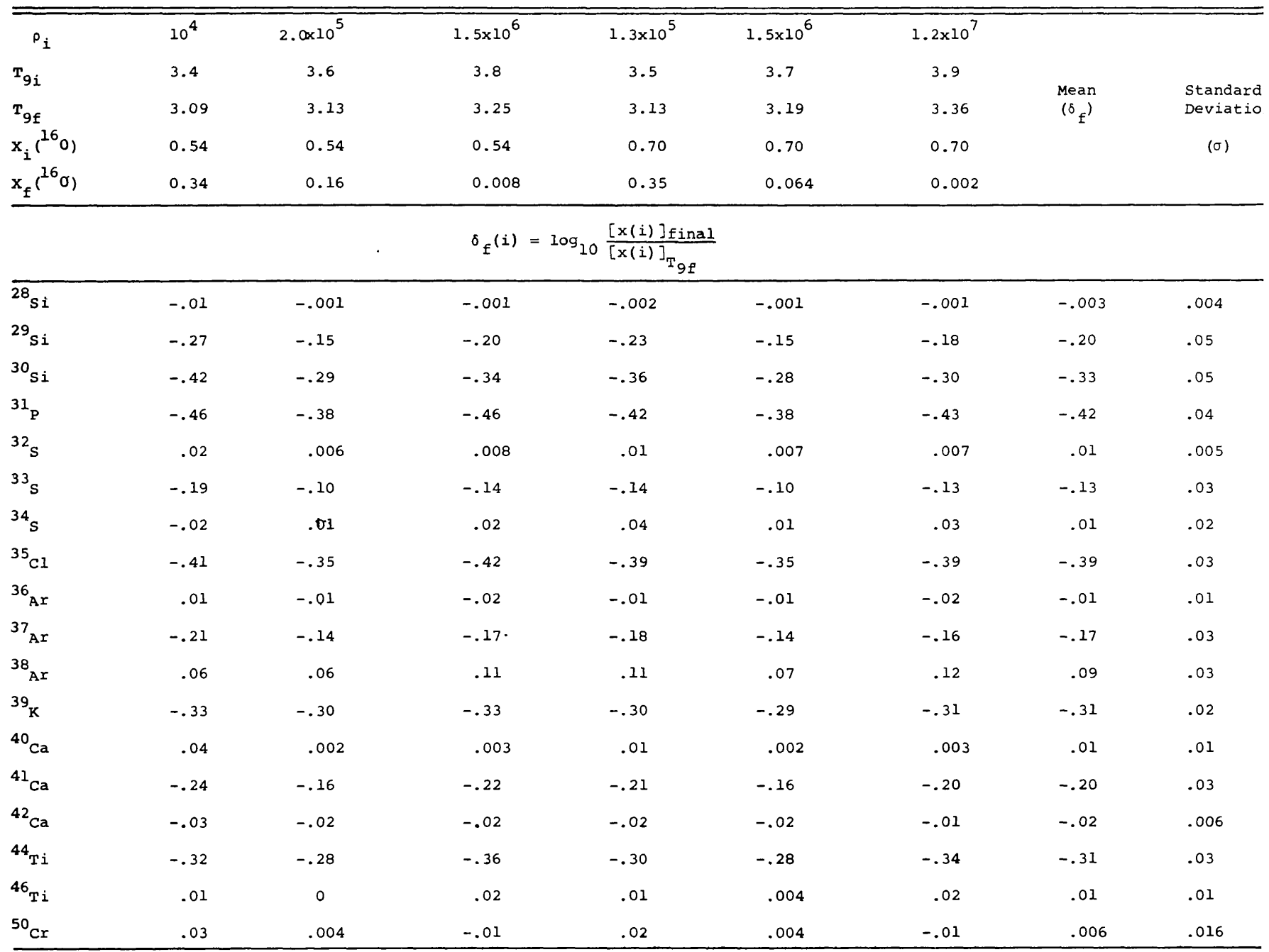

c) Temperature Required for Quasi-Equilibrium for $A \leq 45$

Realization of this sort of quasi-equilibrium will depend upon the evolution taking place at a sufficiently high temperature for a sufficient length of time so that major flows may come into equilibrium. In order to determine a typical time scale as a function of initial conditions we performed a number of calculations using various initial compositions and densities at constant temperatures in the range $3.4 \leq T_{9} \leq$ 3.9. The network employed was shown in figure $1 a$. We find a time scale for quasiequilibrium to be attained to within a factor of $2\left(r_{j}<0.3\right)$ in the entire $28 \leq A \leq 45$ mass range that is fairly independent of density and initial composition. Empirically

$$
\tau_{\mathrm{qe}} \simeq 4.0 \times 10^{-2}\left(\frac{T_{9}}{3.6}\right)^{-33.3} \mathrm{~s} .
$$

This formula should be accurate to within about a factor of 3 for any constanttemperature oxygen-burning evolution having peak temperatures in the range $3.4 \leq$ $T_{9} \leq 3.9$. The last links to come into equilibrium are those connecting a group comprised of all species $38 \leq A \leq 45$ with the $28 \leq A<38$ group. The most important reactions are ${ }^{38} \mathrm{Ar}(\gamma, p)^{37} \mathrm{Cl}$ and ${ }^{38} \operatorname{Ar}(\gamma, \alpha)^{34} \mathrm{~S}$, both of which have rates 
whose temperature dependence is similar to the inverse of equation (21). The explosive situation differs in that the burning time is limited by the expansion. Empirically we have found in explosive expansions that the bulk of the oxygen burning is limited to roughly the first 10 percent of the expansion time $\tau_{\mathrm{HD}}$, during which time the temperature has dropped no more than a few perrcent. To achieve quasi-equilibrium, therefore, the burning time, say $0.1 \tau_{\mathrm{HD}}$, must exceed the time $\tau_{\mathrm{ge}}\left(T_{9 i}\right)$ required for quasi-equilibrium to be established at the initial temperature; that is

$$
0.1 \tau_{\mathrm{HD}}=44.6 \times \rho_{i}{ }^{-1 / 2} \mathrm{~s} \geq \tau_{\mathrm{qe}}\left(T_{9 i}\right),
$$

which with the aid of equation (21) becomes

$$
\begin{aligned}
T_{9 i} & \geqslant\left[6.4 \times 10^{14}\left(\rho_{i} / \chi^{2}\right)^{1 / 2}\right]^{1 / 33.3}, \\
& =3.5\left(\frac{\rho_{i}}{10^{6} \chi^{2}}\right)^{1 / 66.6} .
\end{aligned}
$$

The region delineated by this equation is shown in figure 7. The restriction is liberal enough that almost any "reasonable" (this word will be further clarified in the next section) conditions will satisfy it. Therefore explosive oxygen burning should attain quasi-equilibrium among species in the $28 \leq A \leqslant 45$ mass range shortly after the beginning of the evolution.

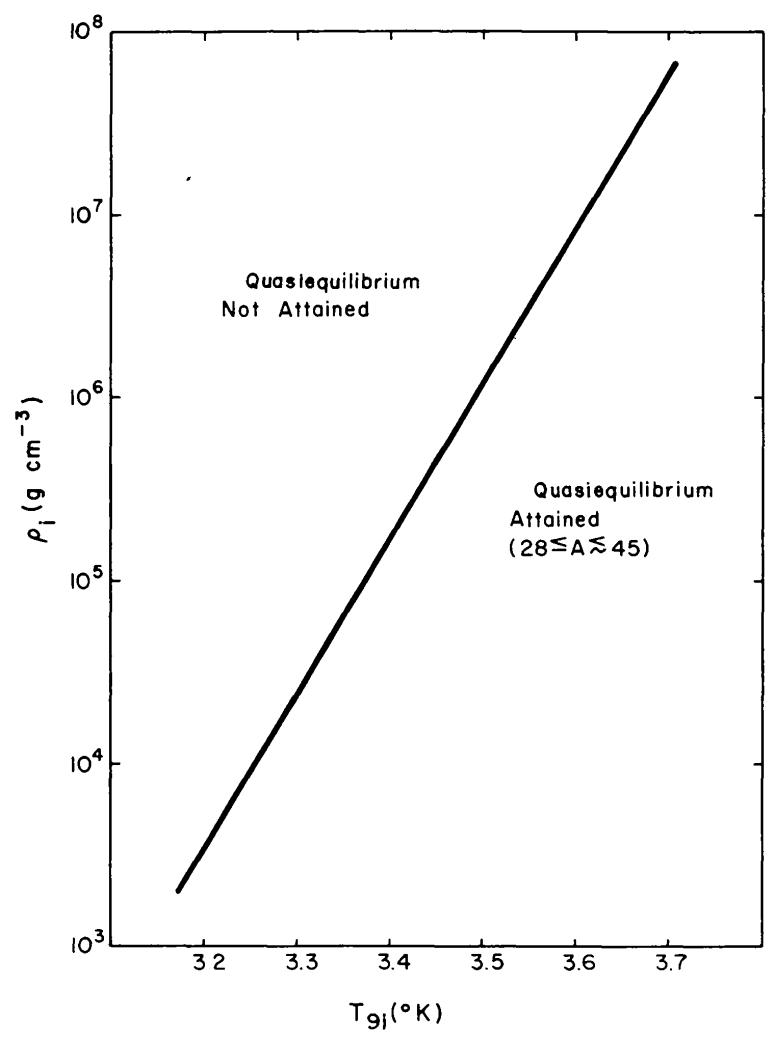

FIG. 7.-Initial conditions leading to quasi-equilibrium in the $28 \leq A \lesssim 44$ mass range. Explosive-oxygen-burning runs employing peak values of the temperature and density which lie to the right of the approximate line shown in the figure should attain quasi-equilibrium in the siliconto-calcium region sometime during the evolution. Runs that employ initial values far to the left of the line should not attain such a consolidated cluster equilibrium. 


\section{d) Particle Densities at Freeze-out}

Given the fact that quasi-equilibrium is attained in the silicon-to-calcium region, it is possible to place restrictions on the free-particle densities at freeze-out. In particular, the abundances of neutrons, protons, and $\alpha$-particles at $T_{9 f}$ should be restricted to those values which can produce the various species in the mass range $28 \leq A \leqslant 45$ in quasi-equilibrium in amounts consistent with their natural abundances. For example, the solar-system ratio of ${ }^{32} \mathrm{~S}$ to ${ }^{28} \mathrm{Si}$ is by number, 0.52 ; therefore to reproduce correctly the sulfur-to-silicon ratio we see from equation (15) that one should have, at $T_{9 f}$,

$$
\left[\frac{Y\left({ }^{32} \mathrm{~S}\right)}{Y\left({ }^{28} \mathrm{Si}\right)}\right]_{\odot}=0.52=C_{32}\left(T_{9 f}\right) \rho N_{\mathrm{A}} Y_{\alpha} .
$$

Accordingly, the alpha density at $T_{9 f}$ necessary to correctly reproduce this solar abundance ratio is given by

$$
\left(\rho X_{\alpha}\right)^{32} \mathrm{~S}^{28} \mathrm{~S} 1=2.08 N_{\mathrm{A}}{ }^{-1} C_{32}{ }^{-1}\left(T_{9 f}\right),
$$

which is a function of the freeze-out temperature only. Similar conditions may be obtained from other ratios that depend only on the alpha density at freeze-out such as: ${ }^{33} \mathrm{~S} /{ }^{29} \mathrm{Si},{ }^{34} \mathrm{~S} /{ }^{30} \mathrm{Si},{ }^{35} \mathrm{Cl} /{ }^{31} \mathrm{P},{ }^{36} \mathrm{Ar} /{ }^{32} \mathrm{~S},{ }^{37} \mathrm{Ar} /{ }^{33} \mathrm{~S},{ }^{38} \mathrm{Ar} /{ }^{34} \mathrm{~S},{ }^{39} \mathrm{~K} /{ }^{35} \mathrm{Cl},{ }^{40} \mathrm{Ca} /{ }^{36} \mathrm{Ar},{ }^{41} \mathrm{Ca} /{ }^{37} \mathrm{Ar}$, ${ }^{42} \mathrm{Ca} /{ }^{38} \mathrm{Ar}$, and ${ }^{44} \mathrm{Ti} /{ }^{40} \mathrm{Ca}$. In doing so the solar abundance of ${ }^{37} \mathrm{Cl}$ is used for ${ }^{37} \mathrm{Ar}$, ${ }^{41} \mathrm{~K}$ for ${ }^{41} \mathrm{Ca}$, and ${ }^{44} \mathrm{Ca}$ for ${ }^{44} \mathrm{Ti}$, respectively, since these are the stable isobars to which these species would decay after explosion. Furthermore, since

$$
\begin{aligned}
& {\left[\frac{Y\left({ }^{(11} \mathrm{K}\right)}{Y\left({ }^{39} \mathrm{~K}\right)}\right]_{\odot}=\left[\frac{Y\left({ }^{41} \mathrm{Ca}\right)}{Y\left({ }^{39} \mathrm{~K}\right)}\right]_{T_{9 f}} \equiv \frac{C_{41}\left(T_{9 f}\right)}{C_{39}\left(T_{9 f}\right)} \frac{Y_{\alpha}}{Y_{p} Y_{n}} \frac{1}{\rho N_{\mathrm{A}}},} \\
& {\left[\frac{Y\left({ }^{37} \mathrm{Cl}\right)}{Y\left({ }^{35} \mathrm{Cl}\right)}\right]_{\odot}=\left[\frac{Y\left({ }^{37} \mathrm{Ar}\right)}{Y\left({ }^{35} \mathrm{Cl}\right)}\right]_{T_{9 f}} \equiv \frac{C_{37}\left(T_{9 f}\right)}{C_{35}\left(T_{9 f}\right)} \frac{Y_{\alpha}}{Y_{p} Y_{n}} \frac{1}{\rho N_{\mathrm{A}}},}
\end{aligned}
$$

where the symbols $Y_{n}$ and $Y_{p}$ represent the abundances of free neutrons and protons at $T_{9 f}$ and since by equation (16)

$$
\frac{Y_{\alpha}}{\rho N_{\mathrm{A}} Y_{n} Y_{p}}=\left[C_{\alpha}\left(T_{9 f}\right) \rho N_{\mathrm{A}} Y_{\alpha}\right]^{1 / 2}
$$

the isotopic ratios of potassium and chlorine will also determine a value of $\rho X_{\alpha}$ as a function only of $T_{9 f}$. Since isotopic ratios may be determined with greater experimental accuracy than elemental ratios, these last two conditions are very restrictive on $\rho X_{\alpha}$. Now an important point comes to light. All these various ratios will give unique values for $\rho X_{\alpha}$ as a function of $T_{9 f}$. Only to the extent that these numbers agree with one another to within the experimental error in the abundance ratio (plus any error that may be introduced by freeze-out effects) can the various species be produced together under the given circumstances. Moreover, the freeze-out temperature which gives the greatest mutual agreement in the predicted values of $\rho X_{\alpha}$ will be the "best value" of the freeze-out temperature for explosive oxygen burning. Values of $T_{9 f}$ which result in the coproduction of very few species must be considered unlikely. Again this implies that such zones should comprise only a small fraction of the material which undergoes explosive oxygen burning. Of course, one might average over several zones of varying $T_{9 f}$ to obtain the solar abundances, creating some of one element under special conditions in one zone, some of another under other conditions. But even if such averaging could yield an acceptable distribution, and there is no 
reason to assume that it would, the large number of species that can be consistently coproduced under certain conditions tends to substantiate the belief that these conditions have astrophysical significance. Moreover, if the species which cannot be produced are known to be created in sizable quantity elsewhere, as will be the case here, this approach takes on added credibility.

Figure $8 a$ shows a plot of the logarithm of the ratio of that value of $\rho X_{\alpha}$ which gives the correct solar-system value for the various elemental and isotopic ratios to the value of $\rho X_{\alpha}$ which gives the solar-system value for ${ }^{32} \mathrm{~S} /{ }^{28} \mathrm{Si}$. The values shown in this figure have not been corrected for freeze-out effects. As seen earlier (see table 3) freeze-out affects the abundances of ${ }^{28} \mathrm{Si},{ }^{32} \mathrm{~S},{ }^{36} \mathrm{Ar}$, and ${ }^{40} \mathrm{Ca}$ very little. The fact that the lines for the ratios ${ }^{32} \mathrm{~S} /{ }^{28} \mathrm{Si},{ }^{36} \mathrm{Ar} /{ }^{32} \mathrm{~S}$, and ${ }^{40} \mathrm{Ca} /{ }^{36} \mathrm{Ar}$ are within their mutual error bars over a large range of freeze-out temperatures shows that these species can be produced together in the proper ratios for almost any reasonable $T_{9 f}$ as long as the value of $\rho X_{\alpha}$ at $T_{9 f}$ is near that value given in figure 9 . It is interesting to note in passing that the new experimental abundance of ${ }^{36} \mathrm{Ar}$ (Eberhart et al. 1970) gives much better agreement for the ratios ${ }^{36} \mathrm{Ar} /{ }^{32} \mathrm{~S}$ and ${ }^{40} \mathrm{Ca} /{ }^{36} \mathrm{Ar}$ than would be obtained using the value interpolated by Cameron (1968).

Since the alpha nuclei comprise the bulk of the mass in the $28 \leq A \leqslant 45$ region, any calculation which correctly reproduces $\rho X_{\alpha}$ at $T_{9 f}$ will often give what appears to be a good representation of the abundances in this region. This explains the success of BCF in matching the abundances of these species at $T_{9}=4.4, \log _{10}\left(\rho X_{\alpha}\right)=4.2$. Since the value of $\rho X_{\alpha}$ predicted by the solar ratio of ${ }^{44} \mathrm{Ca} /{ }^{40} \mathrm{Ce}$ (actually ${ }^{44} \mathrm{Ti} /{ }^{40} \mathrm{Ca}$ ) comes into agreement only at very high freeze-out temperatures, the calculation of BCF almost succeeds in producing ${ }^{44} \mathrm{Ca} /{ }^{40} \mathrm{Ca}$ in the correct ratio whereas the calculation of TA70 does not. However, the comparison of the calculation of BCF to natural abundances has at least two flaws. First, it does not allow for freeze-out effects. In order for a distribution to freeze out at $T_{9 \rho}=4.4$, time scales so short as to be astrophysically implausible must be employed. Second, since the entire region $28 \leq A \leq 62$ is considered to be in quasi-equilibrium in the calculation of $\mathrm{BCF}$, all available neutrons are bound into species in the vicinity of the iron peak. Hence species such as ${ }^{33} \mathrm{~S},{ }^{34} \mathrm{~S}$, ${ }^{37} \mathrm{Ar},{ }^{38} \mathrm{Ar},{ }^{41} \mathrm{Ca}$, and ${ }^{42} \mathrm{Ca}$ are severely underproduced in their calculation.

Figure $8 b$ shows basically the same quantities as figure $8 a$ except that allowance has been made here for the changes in the abundances of species with small mass fractions that occur during freeze-out. These corrections were computed from the information in table 3 . The values of $\rho X_{\alpha}$ given by ${ }^{41} \mathrm{~K} /{ }^{39} \mathrm{~K}$ and ${ }^{37} \mathrm{Cl} /{ }^{35} \mathrm{Cl}$, both accurately measured ratios, now determine a preferred freeze-out temperature range, specifically $3.1 \leq$ $T_{9 f} \leq 3.9$, for explosive oxygen burning. Events which freeze out in this temperature range with the correct value of $\rho X_{\alpha}$ will best reproduce the solar-system distribution in the $28 \leq A \leq 44$ region. In this range the ratios ${ }^{37} \mathrm{Cl} /{ }^{33} \mathrm{~S},{ }^{38} \mathrm{Ar} /{ }^{34} \mathrm{~S},{ }^{39} \mathrm{~K} /{ }^{35} \mathrm{Cl}$, ${ }^{41} \mathrm{~K} /{ }^{37} \mathrm{Cl}$, and ${ }^{42} \mathrm{Ca} /{ }^{38} \mathrm{Ar}$, as well as the ratios of the alpha nuclei, ${ }^{28} \mathrm{Si},{ }^{32} \mathrm{~S},{ }^{36} \mathrm{Ar}$, and ${ }^{40} \mathrm{Ca}$, are reproduced to within the experimental error in their solar-system abundances. The values given by ${ }^{33} \mathrm{~S} /{ }^{29} \mathrm{Si},{ }^{34} \mathrm{~S} /{ }^{30} \mathrm{Si},{ }^{35} \mathrm{Cl} /{ }^{31} \mathrm{P}$, and, as previously mentioned, ${ }^{44} \mathrm{Ca} /{ }^{40} \mathrm{Ca}$, are inconsistent. The failure of the first three ratios is not too surprising since the synthesis of ${ }^{29} \mathrm{Si},{ }^{30} \mathrm{Si}$, and ${ }^{31} \mathrm{P}$ is influenced by explosive carbon burning (Arnett 1969b).

A similar analysis may be performed by considering restrictions on the neutron density. In this case various species, frequently isotopes of the same element, are produced in ratios dependent upon the value of $\rho X_{n}$ at freeze-out, where $X_{n}$ is the neutron mass fraction. For example, the fact that the solar-system ratio for ${ }^{38} \mathrm{Ar} /{ }^{36} \mathrm{Ar}$ is 0.19 by number gives

$$
\left(\rho X_{n}\right)^{38} \mathrm{Ar} /{ }^{36} \mathrm{Ar}=N_{\mathrm{A}}^{-1}\left[0.19 \frac{C_{36}\left(T_{9 f}\right)}{C_{38}\left(T_{9 f}\right)}\right]^{1 / 2},
$$


(a)

O)

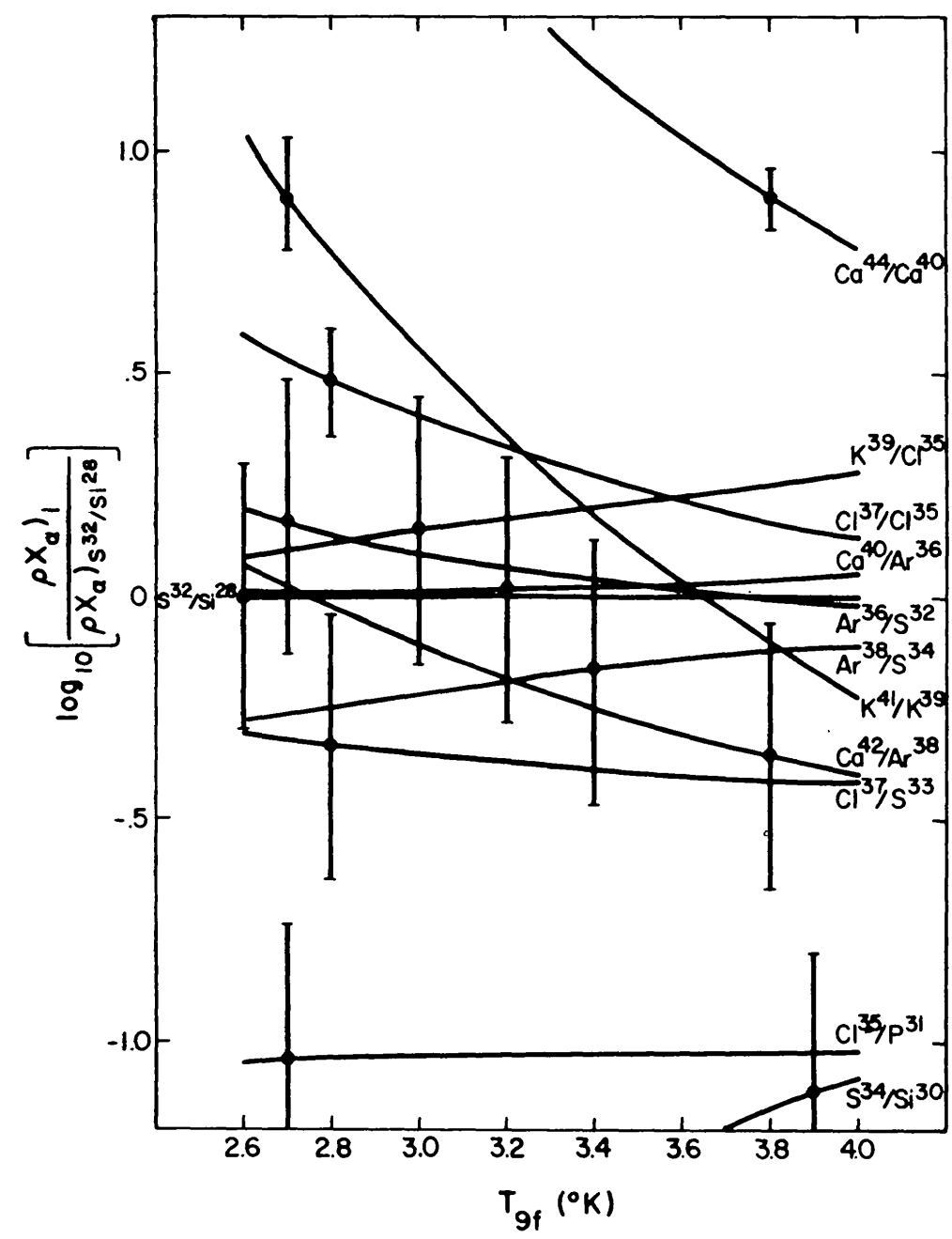

(b)

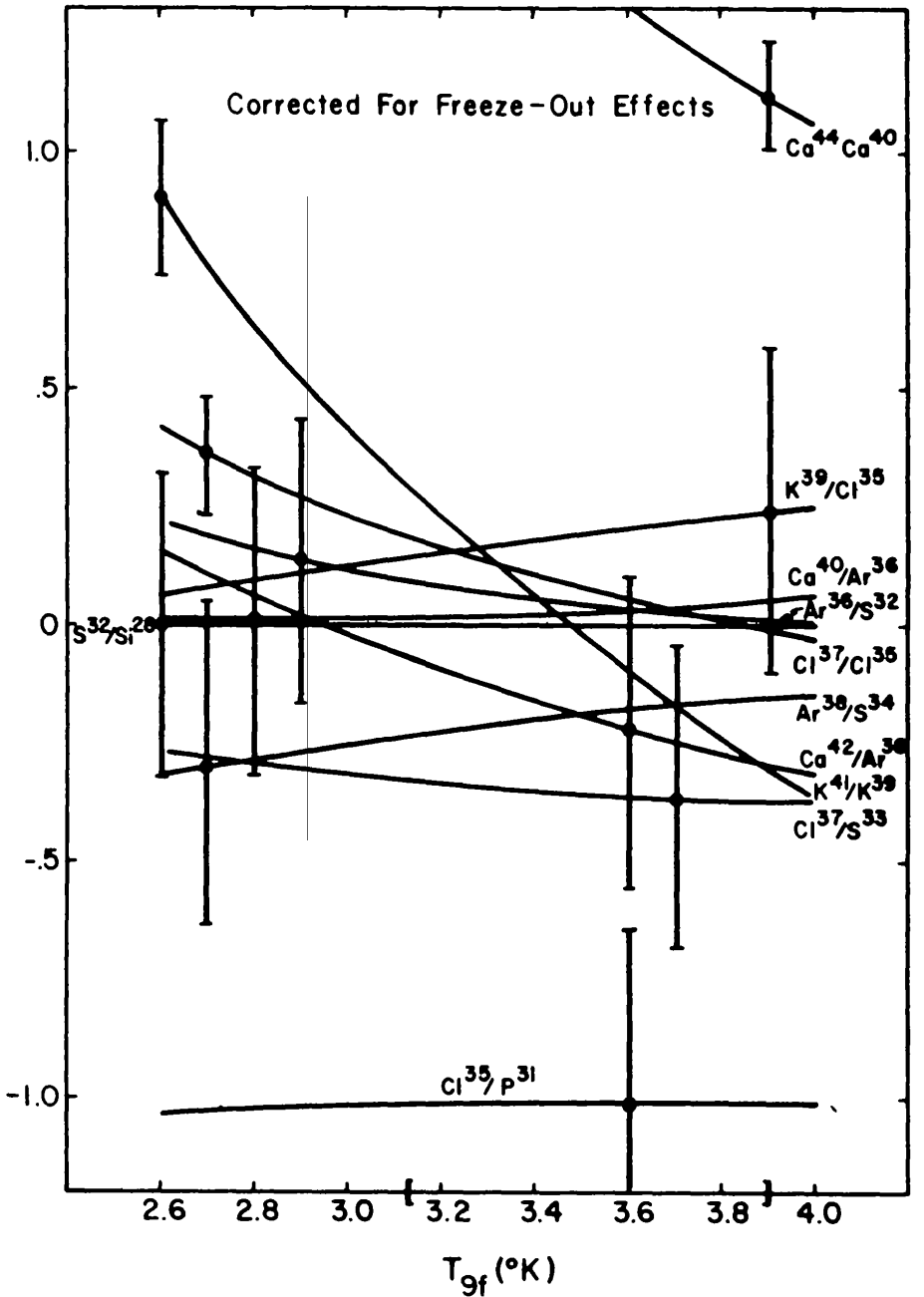

FIG. 8a.- Values of the alpha density during quasi-equilibrium implied by various solar abundance ratios. The logarithm (base 10) of the ratio of that alpha mass density $\rho X_{\alpha}$ required to reproduce the solar ratio of the given species in quasi-equilibrium to that value of $\rho X_{\alpha}$ required to reproduce the ratio ${ }^{32} \mathrm{~S} /{ }^{28} \mathrm{Si}$ correctly is shown as a function of the quasi-equilibrium temperature. Error bars are due to an estimated factor of $2( \pm 0.3$ in the logarithm) uncertainty in the experimental determination of elemental ratios and 15 percent $( \pm 0.06$ in the logarithm) in the determination of isotopic ratios.

Fig. 8b. - Values of the alpha density corrected for freeze-out. The same quantities shown in fig. $8 a$ are shown here corrected for the changes in the various ratios which occur during freeze-out. See also table 3. The error bars now represent the error in the determination of the various ratios plus the standard deviation of the freeze-out correction. An "allowable" range of freeze-out temperatures is shown for which the error bars of many key species which should be produced in explosive-oxygenburning overlap. 


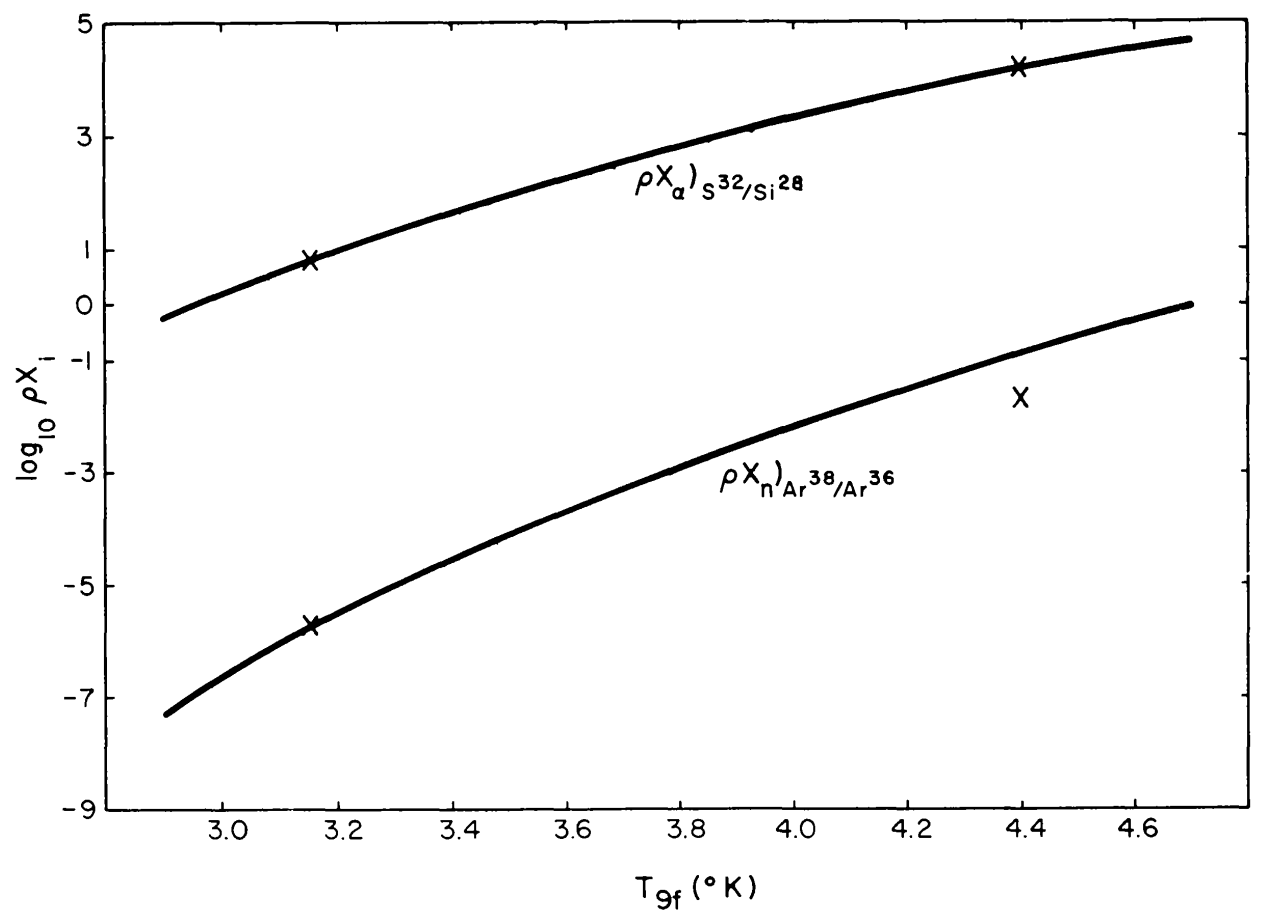

Fig. 9.-Values of the alpha and neutron density implied by the solar ratios of ${ }^{32} \mathrm{~S} /{ }^{28} \mathrm{Si}$ and ${ }^{38} \mathrm{Ar} /{ }^{36} \mathrm{Ar}$ during quasi-equilibrium. The logarithm of the alpha density required to reproduce ${ }^{32} \mathrm{~S} /{ }^{28} \mathrm{Si}$ correctly and the logarithms of the neutron density required to reproduce ${ }^{38} \mathrm{Ar} /{ }^{36} \mathrm{Ar}$ correctly in quasi-equilibrium are shown as functions of the quasi-equilibrium temperature. The crosses at $T_{9}=3.17$ are the freeze-out values of $\rho X_{\alpha}$ and $\rho X_{n}$ obtained in the standard explosiveoxygen-burning run. The crosses at $T_{9}=4.4$ are the values used by BCF to fit the solar distribution of elements in the mass range $28 \leq A \leqslant 62$.

which is a function only of $T_{9 f}$. The value of $\rho X_{n}$ as a function of $T_{9 f}$ required to correctly reproduce ${ }^{38} \mathrm{Ar} /{ }^{36} \mathrm{Ar}$ at $T_{9 f}$ is shown in figure 9. Again, other ratios can be employed to predict various values for $\rho X_{n}$ as functions of $T_{9 f}$. Figure $10 a$ shows the values of $\rho X_{n}$ obtained from the solar-system abundance ratios uncorrected for freeze-out, whereas figure $10 b$ shows the values of $\rho X_{n}$ after the ratios have been corrected for freeze-out. In both figures we have actually plotted the ratio of the required value of $\rho X_{n}$ to the value of $\rho X_{n}$ that reproduces the solar ${ }^{38} \mathrm{Ar} /{ }^{36} \mathrm{Ar}$ abundance ratio; therefore they show which solar-system abundance ratios are in neutron equilibrium with the ${ }^{38} \mathrm{Ar} /{ }^{36} \mathrm{Ar}$ ratio. For a wide range of $T_{9 f}$ the values ${ }^{34} \mathrm{~S} /{ }^{32} \mathrm{~S}$, ${ }^{38} \mathrm{Ar} /{ }^{36} \mathrm{Ar}$, and ${ }^{42} \mathrm{Ca} /{ }^{40} \mathrm{Ca}$ can be consistently reproduced if $\rho X_{n}$ is chosen correctly, but only in the temperature range $3.1 \leq T_{9 f} \leq 4.1$ can the elemental ratios ${ }^{41} \mathrm{~K} /{ }^{40} \mathrm{Ca}$ and ${ }^{37} \mathrm{Cl} /{ }^{36} \mathrm{Ar}$ also be reproduced correctly. This result is in close accord with the range in $T_{9 f}$ required by ratios dependent upon the alpha density. The ratios ${ }^{29} \mathrm{Si} /{ }^{28} \mathrm{Si}$ and ${ }^{30} \mathrm{Si} /{ }^{28} \mathrm{Si}$, on the other hand, are not consistent with the ${ }^{38} \mathrm{Ar} /{ }^{36} \mathrm{Ar}$ ratio for any reasonable value of $T_{9 f}$. These heavy silicon isotopes are in fact probably synthesized in carbon burning (Arnett 1969b).

The results of this discussion then suggest that the species ${ }^{28} \mathrm{Si},{ }^{32} \mathrm{~S},{ }^{34} \mathrm{~S},{ }^{35} \mathrm{Cl},{ }^{37} \mathrm{Cl}$, ${ }^{36} \mathrm{Ar},{ }^{38} \mathrm{Ar},{ }^{39} \mathrm{~K},{ }^{41} \mathrm{~K},{ }^{40} \mathrm{Ca},{ }^{42} \mathrm{Ca}$, and perhaps ${ }^{33} \mathrm{~S}$ can be produced simultaneously by any explosive event which freezes out with values of $\rho X_{n}$ and $\rho X_{\alpha}$ close to those given in figure 9. All the other species which are not produced here (with the exception of $\left.{ }^{44} \mathrm{Ca}\right)$ are very likely synthesized during explosive carbon burning, either from the fuel itself (Arnett 1969b) or by neutron-initiated reactions on heavier seed nuclei (Howard et al. 1972). 
(a)

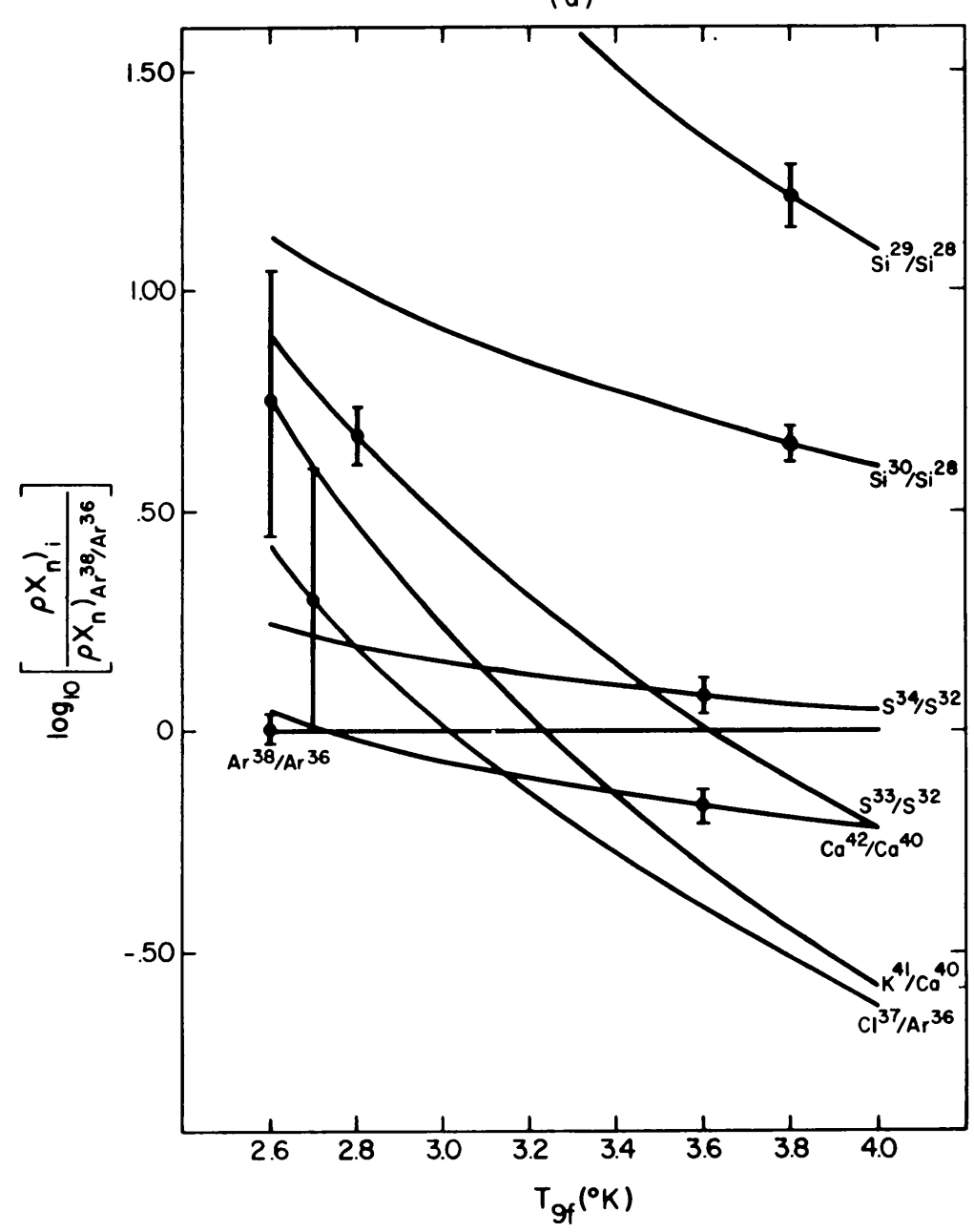

(b)

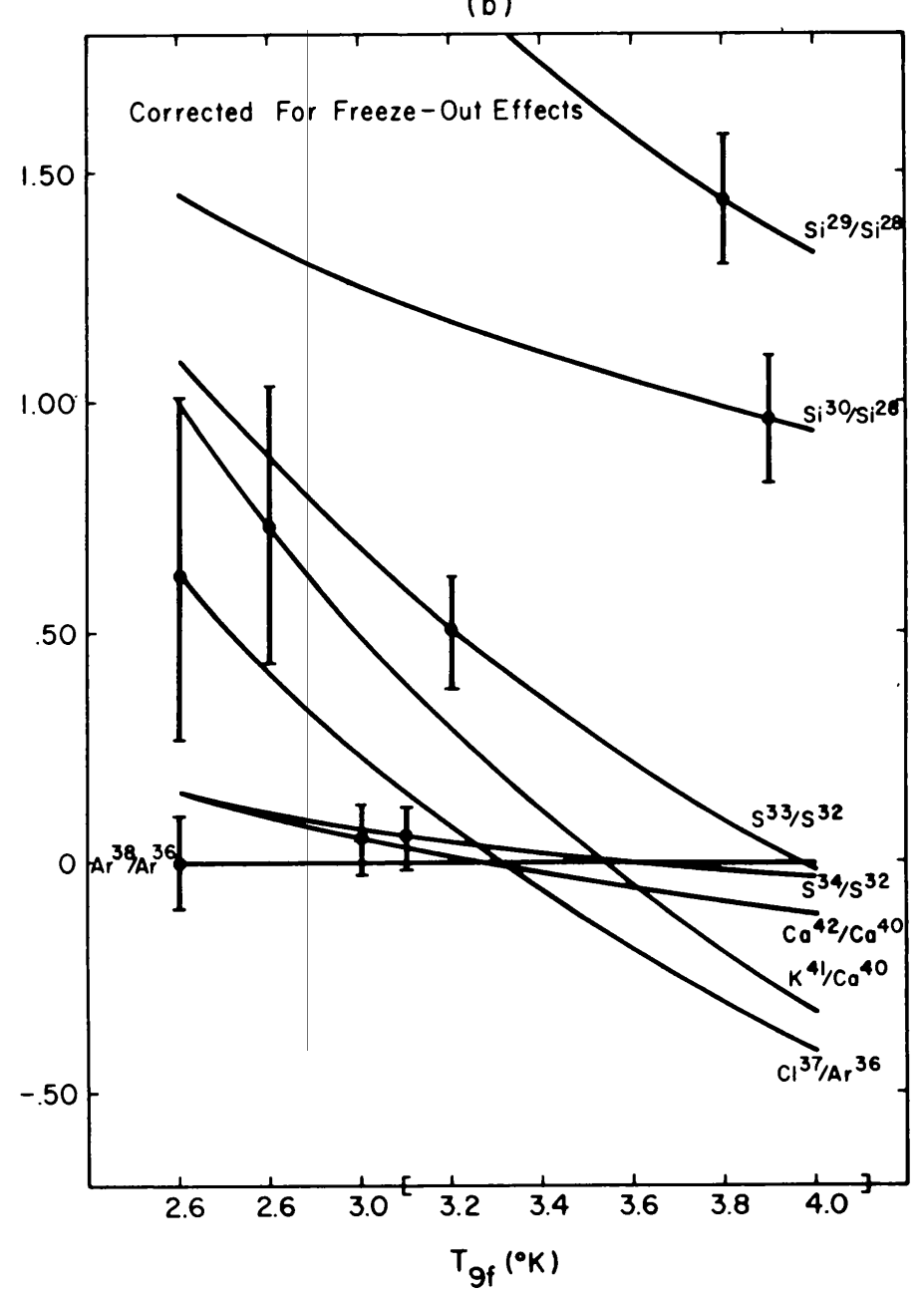

FIG. 10a.-Values of the neutron density during quasi-equilibrium implied by various solar abundance ratios. The logarithm of the ratio of that neutron mass density, $\rho X_{n}$, required to reproduce the solar ratio of the given species in quasi-equilibrium to that value of $\rho X_{n}$ required to reproduce the ratio ${ }^{38} \mathrm{Ar} /{ }^{36} \mathrm{Ar}$ is shown as a function of the quasi-equilibrium temperature. Error bars are due to an estimated factor of 2 uncertainty in the experimental determination of elemental ratios and 15 percent in the determination of isotopic ratios.

Fig. 10b.-Values of the neutron density corrected for freeze-out. The same quantities as shown in fig. 10a are shown here corrected for the changes in the various ratios which occur during freeze-out. See also table 3. The error bars now represent the error in the determination of the various ratios plus the standard deviation of the freeze-out correction. An "allowable" range of freeze-out temperatures is shown for which the error bars of many key species which should be produced in explosive oxygen burning overlap. 


\section{RESTRICTIONS ON THE CONDITIONS FOR EXPLOSIVE OXYGEN BURNING}

The excellent agreement of the final abundance distribution of explosive oxygen burning with the observed solar values shown in figure 2 confirms that we can successfully synthesize these elements in such an event. This is in general agreement with TA70. We now wish to ask a somewhat different question. Given that these elements are due to an explosive event, what limits do the data points (solar abundances) place on the explosion parameters (supernova conditions)? The values of the parameters $T_{9 i}, \rho_{i}, \chi$, and $\left\{X_{i}\right\}$ which yield good agreement are not unique, but they are restricted. One can well imagine a volume in the $n$-dimensional space of all relevant parameters containing only points characterizing explosions in which the nuclei which we would attribute to explosive oxygen burning are produced to within a factor of 2 of their solar values. The dimensionality $n$ of the space would be three plus the number of initial composition variables and cross-section values which can significantly affect the final abundance distribution. Within the context of our assumptions, this $n$-dimensional volume can, in theory at least, be delineated by the set of solar abundances and the "inverse transform" afforded us by our nucleosynthesis theory. The specification of this region has important consequences for the supernova model. We would expect that the thermodynamic history of a typical explosive-oxygen-burning zone would be (at least approximately) specified by parameters contained within the volume. We will not attempt to determine the exact boundaries of the entire volume. Instead we will concentrate only on those parameters which we feel are most critical in an attempt to outline broadly the allowable range of conditions. A more accurate determination would probably not be meaningful due to the simplicity of the model considered here. For explosive oxygen burning we have found the important composition variables to be the initial abundances of ${ }^{16} \mathrm{O},{ }^{24} \mathrm{Mg}$, and ${ }^{28} \mathrm{Si}$ and the amount of neutron enrichment, $\eta$, that has occurred in previous (hydrostatic) burning phases. The values of $\eta$ which we will determine to be "allowed" will have important implications for the presupernova history of the typical zone (see Arnett and Truran 1969; Arnett 1971; and Woosley et al. 1972b). The identity of the species containing the excess neutrons in the initial composition does not matter in these calculations as long as it is lighter than any neutron-rich nuclei (i.e., $Z<N$ ) produced in explosive oxygen burning, hence as long as $Z<16$. This simplification allows us to be somewhat unspecific concerning the initial excess neutrons, but we generally assume them to be contained in a small initial abundance of ${ }^{26} \mathrm{Mg}$. Thus the dimensionality of our physical parameter space is six: $T_{9 i}, \rho_{i}, \eta, \chi, X_{i}\left({ }^{16} \mathrm{O}\right)$, and $X_{i}\left({ }^{24} \mathrm{Mg}\right)$. The initial mass fraction of ${ }^{28} \mathrm{Si}$ is determined by mass conservation.

In addition to the six physical parameters we must also consider the effect of the specific value chosen for certain cross-sections. Since the rates given in the Appendix are only approximately correct, the dimensionality of our allowed space is increased by the number of cross-sections which can have substantial influence on the final abundance distribution. Fortunately this number is found to be small. Due to the quasi-equilibrium character of reactions above ${ }^{28} \mathrm{Si}$, the only reactions whose rates lead to major uncertainties involve lighter species. The reactions which we have found to be especially important are: ${ }^{16} \mathrm{O}(p, \alpha){ }^{13} \mathrm{~N},{ }^{16} \mathrm{O}(\gamma, \alpha){ }^{12} \mathrm{C},{ }^{16} \mathrm{O}+{ }^{16} \mathrm{O},{ }^{12} \mathrm{C}+{ }^{16} \mathrm{O}$, and ${ }^{12} \mathrm{C}+{ }^{12} \mathrm{C}$. The branching ratios for the last three reactions are, however, relatively unimportant; only the total reaction cross-sections seem to matter. For example, two calculations, one of which assumed that the ${ }^{32} \mathrm{~S}$ compound nuclear states produced by ${ }^{16} \mathrm{O}+{ }^{16} \mathrm{O}$ reactions decay solely through the $\alpha$-channel and the other of which assumed the "normal" branching ratios ( III $a$ ) gave results which differed by less than one percent in the final mass fractions of abundant species. Laboratory measurements of the cross-sections for the above five rates in the energy range of interest $\left(3.0 \leq T_{9} \leq 4.0\right)$ would be extremely useful in limiting the allowable conditions. It is perhaps worthy of note that at these temperatures the ${ }^{16} \mathrm{O}(\gamma, \alpha)^{12} \mathrm{C}$ reaction 
occurs primarily through the $9.54\left(1^{-}\right), 9.81\left(2^{+}\right)$, and $10.31\left(4^{+}\right) \mathrm{MeV}$ excited states in the ${ }^{16} \mathrm{O}$ nucleus, not the $7.12\left(1^{-}\right) \mathrm{MeV}$ state which is so important to the ${ }^{12} \mathrm{C}(\alpha, \gamma){ }^{16} \mathrm{O}$ reaction rate during helium burning. Thus the rate of ${ }^{16} \mathrm{O}(\gamma, \alpha){ }^{12} \mathrm{C}$ here is not dependent in any sensitive fashion on the reduced width for alpha emission $\left(\theta_{\alpha}{ }^{2}\right)$ of the $7.12 \mathrm{MeV}$ state which is currently regarded with some uncertainty.

To delimit the allowed burning conditions it is convenient to define the two parameters

$$
\begin{aligned}
\delta_{n} & \equiv \frac{\sum_{i}^{\prime}\left[N_{n}(i)-Z_{p}(i)\right] Y(i)}{\sum_{i}^{\prime} X(i)}, \\
\delta_{\alpha} & \equiv \frac{\frac{1}{4} \sum_{i}^{\prime}[A(i)-28] Y(i)}{\sum_{i}^{\prime} Y(i)},
\end{aligned}
$$

for the set of final abundances

$$
\begin{aligned}
\left\{X_{f}\right\}_{\text {EOB }} \equiv & { }^{28} \mathrm{Si},{ }^{32} \mathrm{~S},{ }^{34} \mathrm{~S},{ }^{35} \mathrm{Cl},{ }^{37} \mathrm{Cl}\left({ }^{37} \mathrm{Ar}\right),{ }^{36} \mathrm{Ar},{ }^{38} \mathrm{Ar},{ }^{39} \mathrm{~K},{ }^{41} \mathrm{~K}\left({ }^{41} \mathrm{Ca}\right), \\
& { }^{40} \mathrm{Ca},{ }^{42} \mathrm{Ca},{ }^{46} \mathrm{Ti},{ }^{50} \mathrm{Cr} .
\end{aligned}
$$

Here the subscript EOB refers to "explosive oxygen burning." These are the nuclei we believe to be due to explosive oxygen burning, and the primed summations extend only over this set $\left\{X_{f}\right\}_{\text {EOB }}$. From the above definitions it is apparent that $\delta_{n}$ is simply the value of $\eta$ for the restricted set $\left\{X_{f}\right\}_{\text {EOB }}$. Because $\eta=\sum_{j} \eta_{j} X_{j}$ we see that $\delta_{n}$ contributes to the total $\eta$ of the gas in proportion to the mass fraction $\left\{X_{f}\right\}_{\text {EOB }}$ of the set. The meaning of $\delta_{\alpha}$ is slightly different. For small $\eta$, each element produced in explosive oxygen burning may be pictured as a ${ }^{28} \mathrm{Si}$ core plus some number (not necessarily integer) of $\alpha$-particles. Then the numerator of equation (30) represents the number of bound $\alpha$-particles (in excess of ${ }^{28} \mathrm{Si}$ cores) contained in the explosive-oxygen-burning set. The denominator is the number of ${ }^{28} \mathrm{Si}$ cores contained in the same set. Thus $\delta_{\alpha}$ is the number of "excess $\alpha$-particles" per ${ }^{28} \mathrm{Si}$ core in the set of abundances $\left\{X_{f}\right\}_{\mathrm{EOB}}$. Then $\delta_{\alpha}$ and $\delta_{n}$ are uniquely specified by a given abundance distribution. Evaluating these quantities using the solar-system abundances that we have adopted for this paper gives $\delta_{n \odot}=1.9 \times 10^{-3}$ and $\delta_{\alpha \odot}=0.60$. These are the values that explosive oxygen burning in nature has evidently produced. For the solar abundances $X_{f}\left({ }^{28} \mathrm{Si}\right)=$ $0.55\left\{X_{f}\right\}_{\mathrm{EOB}}$, so that the effective contribution of this set to the total $\eta$ of the gas is

$$
\eta_{\mathrm{eff} \odot}=\delta_{n \odot}\left\{X_{f}\right\}_{\mathrm{EOB}}=3.5 \times 10^{-3} X_{f}\left({ }^{28} \mathrm{Si}\right),
$$

where $X_{f}\left({ }^{28} \mathrm{Si}\right)$ is its final mass fraction, and the other members of the set $\left\{X_{f}\right\}_{\text {EOB }}$ have been synthesized in their solar ratio to ${ }^{28} \mathrm{Si}$. We have called this $\eta_{\text {eff }}$ because if the only important final species not contained in $\left\{X_{f}\right\}_{\mathrm{EOB}}$ is the unburned ${ }^{16} \mathrm{O}$ fuel, which has $\eta=0$, then $\eta_{\text {eff }}$ is the total value of $\eta$ for the gas.

If the correct solar abundances differ from those of Cameron (1968) with Ar reduced by a factor of 2 , then, of course, the values of $\delta_{n \odot}$ and $\delta_{\alpha \odot}$ also differ. In this regard we note that the measurements of Crawford, Price, and Sullivan (1972) on high-energy particles from solar flares suggest that the abundance sequence $\mathrm{Si}-\mathrm{S}$ Ar-Ca may fall more steeply than it does for the abundances we have adopted. Their measurements suggest, in fact, that the solar values could be as low as $\delta_{n \odot}=0.7 \times$ $10^{-3}$ and $\delta_{\alpha \odot}=0.25$. We point this out because these values are substantially lower than the ones given above, and the reader should, we believe, make a mental reservation concerning any detailed results which depend upon the exact values of these two abundance parameters. Nonetheless we will proceed to use the Cameron based values in our numerical examples that follow. 


\section{a) Restrictions on the Neutron Excess}

Consider the restrictions on $\eta$ imposed by the constraint $\delta_{n}=\delta_{n \odot}$. Since explosive oxygen burning is found to occur on a time scale far too short for weak interactions to appreciably alter $\eta$ but usually on a time scale long enough to convert the initial neutron source seed into species in the set $\left\{X_{f}\right\}_{\mathrm{EOB}}$ and heavier, the initial neutron excess of the material should equal $\eta_{\text {eff }}$ plus enough neutrons to account for any additional neutron sinks other than those contained in the set $\left\{X_{f}\right\}_{\mathrm{EOB}}$. The important additional neutron sink is ${ }^{54} \mathrm{Fe}$. At low temperature little penetration through the ${ }^{40} \mathrm{Ca}$ barrier to larger atomic weight occurs; then $\eta=\eta_{\text {eff }}$ is the correct initial neutron excess for producing $\left\{X_{f}\right\}_{\mathrm{EOB}}$. But at higher temperatures, specifically for $T_{9 i} \geqslant 3.6$, larger and larger amounts of ${ }^{54} \mathrm{Fe}$ are found to be produced until, at $T_{9 i} \simeq 3.9$, a value of $\eta$ sufficient to give the abundances in $\left\{X_{f}\right\}_{E O B}$ correctly would result in the production of an entire solar abundance of ${ }^{54} \mathrm{Fe}$ relative to ${ }^{28} \mathrm{Si}$. This result is almost certainly unacceptable, because nucleosynthesis from more advanced burning also produces a large amount of ${ }^{54} \mathrm{Fe}$ and is generally assumed (see e.g., Arnett and Clayton 1970) to be the source of ${ }^{54} \mathrm{Fe}$. We therefore regard $T_{9 i}=3.9$ as being an approximate upper bound to the peak temperature at which nature has explosively synthesized the set $\left\{X_{f}\right\}_{\text {EOB }}$. Let us define an upper bound to $\eta$ by the requirement that it be capable of providing the excess neutrons for the synthesis of no more than half of the ${ }^{54} \mathrm{Fe}$ along with the set $\left\{X_{f}\right\}_{\mathrm{EOB}}$ in its solar ratios

$$
\eta_{\max }=\eta_{\text {eff } \odot}+\frac{28-26}{54} X_{f}\left({ }^{54} \mathrm{Fe}\right)=\eta_{\text {eff } \odot}+\frac{2}{54} \frac{1}{2}\left[\frac{X_{\odot}\left({ }^{54} \mathrm{Fe}\right)}{X_{\odot}\left({ }^{28} \mathrm{Si}\right)}\right] X_{f}\left({ }^{28} \mathrm{Si}\right),
$$

and using $X_{\odot}\left({ }^{54} \mathrm{Fe}\right)=0.11 X_{\odot}\left({ }^{28} \mathrm{Si}\right)$ and equation (31) this becomes $\eta_{\max }=5.5 \times$ $\left.10^{-3} X_{f}{ }^{28} \mathrm{Si}\right)$. Since ${ }^{28} \mathrm{Si}$ contains about 55 percent of all the mass in the solar-abundance set $\left\{X_{f}\right\}_{\mathrm{EOB}}$ the maximum acceptable final mass fraction of ${ }^{28} \mathrm{Si}$ (assuming ${ }^{16} \mathrm{O}$ exhaustion) is 0.55 . This implies an upper bound $\eta_{\max } \simeq 3.0 \times 10^{-3}$. Values of $\eta$ substantially higher than this will result in an overproduction of either ${ }^{54} \mathrm{Fe}$ or the neutron-rich species of the set $\left\{X_{f}\right\}_{\mathrm{EOB}}$.

We may also estimate the lower bound to acceptable values of $\eta$. As discussed previously, substantial production of species heavier than ${ }^{28} \mathrm{Si}$ requires the depletion of the initial ${ }^{24} \mathrm{Mg}$ abundance. Thus a minimum mass fraction $7 / 6 X_{i}\left({ }^{24} \mathrm{Mg}\right)+X_{i}\left({ }^{28} \mathrm{Si}\right)$, where $X_{i}\left({ }^{24} \mathrm{Mg}\right)$ and $X_{i}\left({ }^{28} \mathrm{Si}\right)$ are the initial mass fractions, must be contained in the set $\left\{X_{f}\right\}_{\mathrm{EOB}}$; therefore,

$$
X_{\min }\left({ }^{28} \mathrm{Si}\right)=0.55\left[\frac{7}{6} X_{i}\left({ }^{24} \mathrm{Mg}\right)+X_{i}\left({ }^{28} \mathrm{Si}\right)\right] .
$$

For the composition of equation (9) this gives a value of $\left.X_{\min }{ }^{28} \mathrm{Si}\right)=0.27$. The minimum $\eta$ produces no ${ }^{54} \mathrm{Fe}$, hence from equation (31) we have $\eta_{\min } \simeq 9.0 \times 10^{-4}$. Below this value the neutron-rich species of $\left\{X_{f}\right\}_{\mathrm{EOB}}$ will be underproduced even if no ${ }^{54} \mathrm{Fe}$ is produced.

In order to verify the accuracy of the above approximations explosive calculations were performed using the network shown in figure $1 a$ for a variety of initial $\eta$ 's at an initial temperature $T_{9 i}=3.6$ and density $\rho_{i}=3.0 \times 10^{5}$. The initial composition employed was that of equation (9) with $\eta=2.0 \times 10^{-4}, 6.0 \times 10^{-4}, 2.0 \times 10^{-3}$, and $6.0 \times 10^{-3}$. The results are shown in figure 11. In this figure a cross denotes the point where the species in question is produced in the correct (solar system) ratio to ${ }^{28} \mathrm{Si}$. Angle brackets delineate an allowable range in $\eta$ for the production of the given species to within a factor of 2 of its solar value. Only a narrow band of $\eta$ between $1.4 \times 10^{-3}$ and $2.0 \times 10^{-3}$ seems to allow the concurrent production of all of the neutron-rich species in the set $\left\{X_{f}\right\}_{\mathrm{EOB}}$. This result agrees reasonably well with the optimum value of $\eta$ of $1.4 \times 10^{-3}$ that one would predict noting that no ${ }^{54} \mathrm{Fe}$ is produced and using 


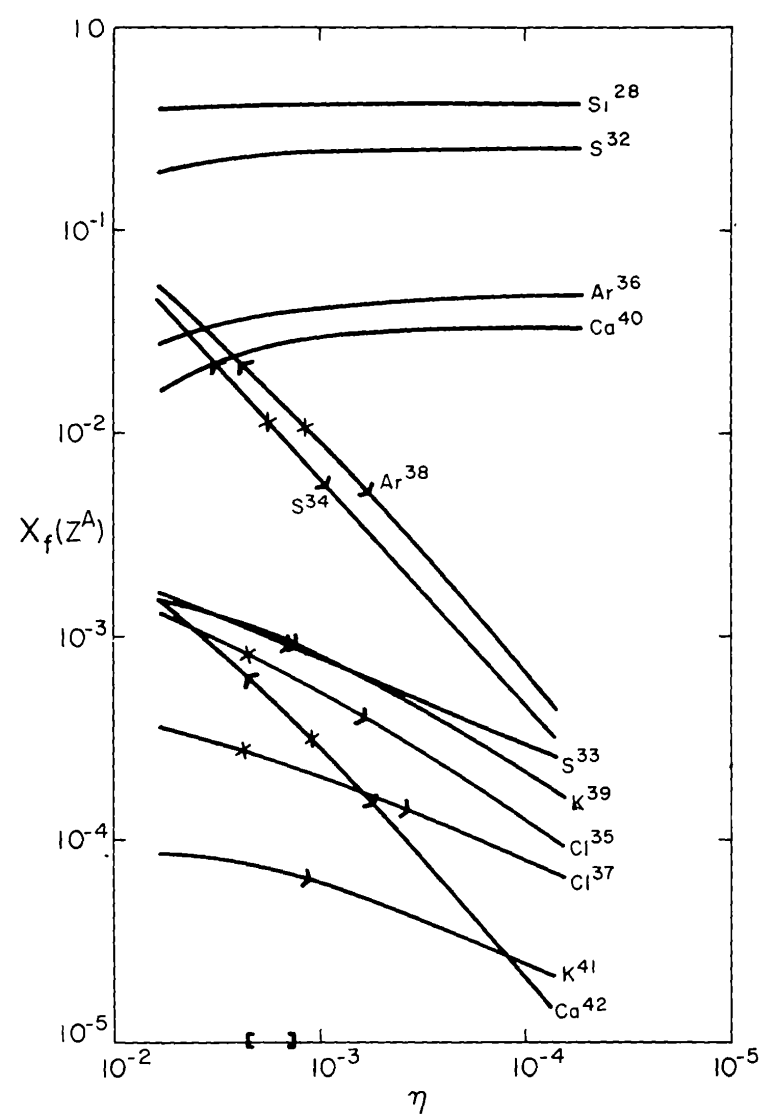

Fig. 11. - Variation of $\eta$ in explosive oxygen burning. The final abundances of several key species are shown as functions of the $\eta$ employed in the "standard" explosive-oxygen-burning run; a cross denotes the point where the species is produced in the correct amount to account for its solar abundance with respect to ${ }^{28} \mathrm{Si}$. Angle brackets denote a range in $\eta$ for which the species is produced to within a factor of 2 of its solar value. Brackets on the $\eta$-axis delimit a range in $\eta$ for which all the species shown may be produced to within a factor of 2 of their solar value.

equation (31) and a final silicon mass fraction of 0.41 , which is the result of explosive oxygen burning under these conditions. The fact that the curves for elements with the same number of excess neutrons are parallel lines (recall that ${ }^{37} \mathrm{Cl}$ is really ${ }^{37} \mathrm{Ar},{ }^{41} \mathrm{~K}$ is ${ }^{41} \mathrm{Ca}$, etc.) follows naturally from the quasi-equilibrium nature of explosive oxygen burning, although it is not in itself a sufficient proof of quasi-equilibrium.

\section{b) Restriction on the Alpha Excess}

What restrictions can be imposed on the quantity $\delta_{\alpha}$ defined in equation (30)? As mentioned previously the set $\left\{X_{f}\right\}_{\mathrm{EOB}}$ in its solar-abundance ratios has a value of $\delta_{\alpha}$ in the vicinity of 0.60 . For values of $\delta_{\alpha}$ larger than this the species in the set $\left\{X_{f}\right\}_{\mathrm{EOB}}$ that are heavier than ${ }^{28} \mathrm{Si}$ are overproduced; for smaller values of $\delta_{\alpha}$ they will be underproduced. The final abundance of ${ }^{40} \mathrm{Ca}$ is particularly sensitive to the value of $\delta_{\alpha}$ since it is one of the heaviest species in the group $\left\{X_{f}\right\}_{\mathrm{EOB}}$. It is empirically found that whenever $\delta_{\alpha}>0.70$, the species ${ }^{40} \mathrm{Ca}$ is overproduced by a factor of 2 or more relative to ${ }^{28} \mathrm{Si}$. Similarly, for $\delta_{\alpha} \leqslant 0.40$ we find that ${ }^{40} \mathrm{Ca}$ is underproduced by a factor of 2 or more. We repeat for emphasis that for $\delta_{\alpha} \simeq 0.60$ (and of course $\eta$ of the right value), all species in the set $\left\{X_{f}\right\}_{\mathrm{EOB}}$ including ${ }^{40} \mathrm{Ca}$, are produced in the proper ratio to ${ }^{28} \mathrm{Si}$ to within a factor of 2 . This is due to the quasi-equilibrium nature of flows among species from ${ }^{28} \mathrm{Si}$ to ${ }^{45} \mathrm{Ti}$. The values of $\delta_{n}$ and $\delta_{\alpha}$ at the freeze-out temperature determine the ratios of the abundances of all species in the group to ${ }^{28} \mathrm{Si}$. The freeze-out 
temperature is fairly constant in explosive oxygen burning, usually in the range $3.1 \leqslant T_{9 f} \leqslant 3.3$. It is characteristic of the quasi-equilibrium distribution in this temperature range that if $\delta_{n}$ and $\delta_{\alpha}$ assume their solar values the final abundance distribution will closely resemble the solar distribution. Hence the criterion which we will employ is that good agreement with solar values may be obtained if and only if $0.40 \leq \delta_{\alpha} \leq 0.70$ and the best set of parameters will be that contour for which $\delta_{\alpha}=0.60$.

The actual value of $\delta_{\alpha}$ for the final distribution will be a complicated function of (1) the initial composition, (2) how much oxygen is burned, and (3) the branching among the various modes for destroying oxygen. These last two quantities will in turn be functions of the peak temperature and density, time scale, and values employed for the five critical nuclear reaction rates. As discussed previously, there are four major ways of consuming ${ }^{16} \mathrm{O}:$ (1) the fusion of two oxygen nuclei, ${ }^{16} \mathrm{O}+{ }^{16} \mathrm{O} \rightarrow\left({ }^{32} \mathrm{~S}\right)^{*} \rightarrow$ various final states; (2) the fusion of oxygen with a ${ }^{12} \mathrm{C}$ nucleus produced by either ${ }^{16} \mathrm{O}(\gamma, \alpha){ }^{12} \mathrm{C}$ or ${ }^{16} \mathrm{O}(p, \alpha){ }^{13} \mathrm{~N}(\gamma, p){ }^{12} \mathrm{C}$, i.e., ${ }^{12} \mathrm{C}+{ }^{16} \mathrm{O} \rightarrow\left({ }^{28} \mathrm{Si}\right) * \rightarrow$ various final states; $(3)$ the photodisintegration of ${ }^{16} \mathrm{O}$ to ${ }^{12} \mathrm{C}$; and (4) the conversion of ${ }^{16} \mathrm{O}$ to ${ }^{12} \mathrm{C}$ and an $\alpha$-particle by a catalytic proton, i.e., ${ }^{16} \mathrm{O}(p, \alpha){ }^{13} \mathrm{~N}(\gamma, p)^{12} \mathrm{C}$. In terms of ${ }^{28} \mathrm{Si}$ cores and "excess $\alpha$-particles" the first two processes convert two ${ }^{16} \mathrm{O}$ nuclei into one core and one excess $\alpha$-particle via

mode 1:

$$
{ }^{16} \mathrm{O}+{ }^{16} \mathrm{O} \rightarrow{ }^{26} \mathrm{Si}+\alpha "
$$

and (because the ${ }^{12} \mathrm{C}$ nuclei are produced by oxygen disintegration)

mode 2:

$$
{ }^{16} \mathrm{O}+{ }^{16} \mathrm{O} \rightarrow{ }^{16} \mathrm{O}+{ }^{12} \mathrm{C}+\alpha \rightarrow{ }^{\text {“28 }} \mathrm{Si}+\alpha " .
$$

Some of the ${ }^{12} \mathrm{C}$ that is produced by oxygen disintegration is not destroyed by the reaction ${ }^{12} \mathrm{C}+{ }^{16} \mathrm{O}$. In that case the last two modes of ${ }^{16} \mathrm{O}$ destruction result in chains of reactions that yield only $\alpha$-particles and no ${ }^{28} \mathrm{Si}$ cores-principally by the processes

mode 3:

$$
\begin{aligned}
& \text { (a) }{ }^{16} \mathrm{O}(\gamma, \alpha){ }^{12} \mathrm{C}\left({ }^{12} \mathrm{C}, \alpha\right)^{20} \mathrm{Ne}(\gamma, \alpha)^{16} \mathrm{O}, \\
& \text { (b) }{ }^{16} \mathrm{O}(\gamma, \alpha)^{12} \mathrm{C}\left({ }^{12} \mathrm{C}, p\right)^{23} \mathrm{Na}(p, \alpha)^{20} \mathrm{Ne}(\gamma, \alpha)^{16} \mathrm{O}, \\
& \text { (c) }{ }^{16} \mathrm{O}(\gamma, \alpha)^{12} \mathrm{C}\left({ }^{12} \mathrm{C}, n\right)^{23} \mathrm{Mg}(n, \alpha){ }^{20} \mathrm{Ne}(\gamma, \alpha)^{16} \mathrm{O}, \\
& \text { (d) }{ }^{16} \mathrm{O}(\gamma, \alpha)^{12} \mathrm{C}(\gamma, \alpha) 2 \alpha ;
\end{aligned}
$$

and

mode 4: $\quad{ }^{16} \mathrm{O}(p, \alpha){ }^{13} \mathrm{~N}(\gamma, p){ }^{12} \mathrm{C}$ replaces ${ }^{16} \mathrm{O}(\gamma, \alpha){ }^{12} \mathrm{C}$ in mode 3 .

Except for the small leak due to ${ }^{12} \mathrm{C}$ photodisintegration, modes 3 and 4 result in conversion of two ${ }^{16} \mathrm{O}$ nuclei into four $\alpha$-particles and an ${ }^{16} \mathrm{O}$ nucleus. Thus the effect of each one is ${ }^{16} \mathrm{O} \rightarrow 4 \alpha$. Now suppose that during a given explosive-oxygen-burning evolution an amount $\Delta X_{16}$ is consumed; that is

$$
\Delta X_{16}=X_{i}\left({ }^{16} \mathrm{O}\right)-X_{f}\left({ }^{16} \mathrm{O}\right) \text {, }
$$

and that a fraction $f$ of this ${ }^{16} \mathrm{O}$ is consumed by modes 1 or 2 which result in the net production of one $\alpha$-particle per ${ }^{28} \mathrm{Si}$ core; i.e., either ${ }^{16} \mathrm{O}+{ }^{16} \mathrm{O}$ or ${ }^{12} \mathrm{C}+{ }^{16} \mathrm{O}$. The remainder of the oxygen is converted into $\alpha$-particles by modes 3 and 4 . Then the total alpha-mass-fraction excess created during the run is defined to be

$$
\begin{aligned}
\Delta X_{\alpha} & =\frac{1}{8} f \Delta X_{16}+(1-f) \Delta X_{16}, \\
& =\left(1-\frac{7}{8} f\right) \Delta X_{16} \quad 0<f<1 .
\end{aligned}
$$


Let us assume an initial composition of ${ }^{16} \mathrm{O},{ }^{24} \mathrm{Mg}$, and ${ }^{28} \mathrm{Si}$ (the small abundance of ${ }^{26} \mathrm{Mg}$ can be included in ${ }^{24} \mathrm{Mg}$ for purposes of this discussion). If we wish to produce a final abundance distribution of given $\delta_{\alpha}$ then the above $\alpha$-particle excess must be sufficient to (1) convert all initial ${ }^{24} \mathrm{Mg}$ to ${ }^{28} \mathrm{Si}$ and (2) provide an $\alpha$-particle excess of $\delta_{\alpha}$ per ${ }^{28} \mathrm{Si}$ core for all the ${ }^{28} \mathrm{Si}$ that is created during the evolution as well as all initial ${ }^{28} \mathrm{Si}$. Then we must have

$$
\Delta X_{\alpha}=\frac{1}{6} X_{i}\left({ }^{24} \mathrm{Mg}\right)+\frac{\delta_{\alpha}}{7} X_{t}\left({ }^{28} \mathrm{Si}\right),
$$

where $X_{i}\left({ }^{24} \mathrm{Mg}\right)$ is the initial mass fraction of ${ }^{24} \mathrm{Mg}$ and $X_{t}\left({ }^{28} \mathrm{Si}\right)$ is the total mass fraction of all ${ }^{28} \mathrm{Si}$ cores in the final distribution. Let $\phi$ be the ratio by mass fraction of ${ }^{24} \mathrm{Mg}$ to ${ }^{28} \mathrm{Si}$ in the initial composition; i.e., $\phi \equiv X_{i}\left({ }^{24} \mathrm{Mg}\right) / X_{i}\left({ }^{28} \mathrm{Si}\right)$. Then

$$
\begin{aligned}
X_{i}\left({ }^{24} \mathrm{Mg}\right) & =\frac{\phi}{1+\phi}\left[1-X_{i}\left({ }^{16} \mathrm{O}\right)\right], \\
X_{i}\left({ }^{28} \mathrm{Si}\right) & =\frac{1}{1+\phi}\left[1-X_{i}\left({ }^{16} \mathrm{O}\right)\right],
\end{aligned}
$$

and because ${ }^{28} \mathrm{Si}$ cores, once created, are preserved throughout oxygen burning

$$
\begin{aligned}
X_{t}\left({ }^{28} \mathrm{Si}\right) & =\frac{7}{8} f \Delta X_{16}+\frac{7}{6} X_{i}\left({ }^{24} \mathrm{Mg}\right)+X_{i}\left({ }^{28} \mathrm{Si}\right), \\
& =\frac{7}{8} f \Delta X_{16}+\left(\frac{7 \phi+6}{6 \phi+6}\right)\left[1-X_{i}\left({ }^{16} \mathrm{O}\right)\right]
\end{aligned}
$$

Solving equations (34) through (38) we have for the fraction of ${ }^{16} \mathrm{O}$ that must burn by ${ }^{12} \mathrm{C}+{ }^{16} \mathrm{O}$ or ${ }^{16} \mathrm{O}+{ }^{16} \mathrm{O}$

where

$$
f=\left(\frac{8}{7+\delta_{\alpha}}\right)\left\{\dot{K}\left[X_{i}\left({ }^{16} \mathrm{O}\right)-1\right]+\Delta X_{16}\right\}\left(\frac{1}{\Delta X_{16}}\right),
$$

$$
K=\frac{7 \phi\left(\delta_{\alpha}+1\right)+6 \delta_{\alpha}}{42(\phi+1)} .
$$

The restriction that $f>0$ requires that

$$
X_{i}\left({ }^{16} \mathrm{O}\right)>\frac{K+X_{f}\left({ }^{16} \mathrm{O}\right)}{K+1}>\frac{K}{K+1} .
$$

This equation provides, for various values of the ${ }^{24} \mathrm{Mg}$ to ${ }^{28} \mathrm{Si}$ ratio, the minimum mass fraction of ${ }^{16} \mathrm{O}$ that must be consumed in explosive oxygen buring in order to achieve $\delta_{\alpha}=0.60$ as required by the solar-abundance data. These minimum mass fractions are given in table 4 . For compositions that have initial oxygen mass fractions as small as the entries in table 4 , all of the ${ }^{16} \mathrm{O}$ would have to be converted into $\alpha$-particles in order to satisfy the $\delta_{\alpha}$ requirements of the large initial abundances of ${ }^{24} \mathrm{Mg}$ and ${ }^{28} \mathrm{Si}$. In this limiting case none of the ${ }^{16} \mathrm{O}$ could burn by processes like ${ }^{16} \mathrm{O}+{ }^{16} \mathrm{O}$ or ${ }^{12} \mathrm{C}+{ }^{16} \mathrm{O}$. In the light of figure 4 this would require a very low density of the typical zone. As we shall see shortly such low densities are unlikely; therefore, the mixed modes of oxygen consumption require oxygen mass fractions a good bit higher than the minimum values of table 4 . The requirement that $f<1$ can be used 
TABLE 4

MINIMUM ${ }^{16} \mathrm{O}$ MASS FRACTIONS FOR $\delta_{\alpha}=0.6$

\begin{tabular}{ccccc}
\hline \hline & \multicolumn{5}{c}{$\Phi$} \\
\cline { 2 - 5 } & 0 & 1 & 2 & $\infty$ \\
\hline$X_{\min \left({ }^{16} \mathrm{O}\right)} \ldots \ldots$ & 0.08 & 0.15 & 0.17 & 0.21 \\
\hline
\end{tabular}

to give an upper limit to the amount of ${ }^{16} \mathrm{O}$ than can burn during a typical explosion,

$$
\Delta X_{16}<\frac{8 K\left[1-X_{i}\left({ }^{16} \mathrm{O}\right)\right]}{1-\delta_{\alpha}} .
$$

It is of some interest to inquire as to the limits imposed by this result on the initial composition for those cases in which all of the initial abundance of ${ }^{16} \mathrm{O}$ is consumed in the explosion, i.e., $X_{f}\left({ }^{16} \mathrm{O}\right)=0$. This is approximately the case as the density becomes very large. At high density the processes ${ }^{16} \mathrm{O}+{ }^{16} \mathrm{O}$ and ${ }^{12} \mathrm{C}+{ }^{16} \mathrm{O}$ dominate over other means of destroying ${ }^{16} \mathrm{O}$; hence $f$ approaches unity. But since both these reactions occur at a rate per unit mass that is linearly dependent upon $\rho$ and occur for a time that decreases only as $\rho^{-1 / 2}$ (see eq. [2]), increasing $\rho$ at a given temperature increases $\Delta X_{16}$ to a point where almost all of the oxygen is burned. When this'occurs, $\Delta X_{16}=X_{i}\left({ }^{16} \mathrm{O}\right)$ and equation (41) gives us an upper limit to the initial oxygen abundance as a function of $\phi$ and $\delta_{\alpha}$. Let us again take $\delta_{\alpha}=0.60$. Then we obtain the limits on the initial ${ }^{16} \mathrm{O}$ abundance as a function of the ${ }^{24} \mathrm{Mg}$ to ${ }^{28} \mathrm{Si}$ ratio given in table 5. These particular compositions are of special interest because they are the only ones which should be capable of producing the solar distribution at very high density. Indeed, for these compositions the upper limit on the peak density in explosive oxygen burning is, at least within the context of this simple discussion, unbounded. This point deserves emphasis: a zone with the above compositions which burns at a density large enough that $f \simeq 1$ will give the solar distribution regardless of other criteria like the peak temperature, time scale, exact value of the peak density, etc., as long as (1) the temperature is low enough so that substantial buildup of ${ }^{54} \mathrm{Fe}$ does not occur and (2) the temperature-time-scale combination is sufficient to allow quasi-equilibrium to be established in the silicon-to-calcium region and most of the ${ }^{16} \mathrm{O}$ to be burned. This unbounding of the peak-density parameter will be interesting in light of the restrictions on supernova energetics which we will be discussing shortly. As a rather obvious corollary to this discussion it may be added that compositions more oxygen-rich than those given by equation (41) with $X_{f}\left({ }^{16} \mathrm{O}\right)=0$ cannot burn all their oxygen without overproducing ${ }^{40} \mathrm{Ca}$ relative to ${ }^{28} \mathrm{Si}$. This fact will severely limit the allowed parameters (e.g., peak temperature) for such compositions. For compositions intermediate to $X_{\min }\left({ }^{16} \mathrm{O}\right)$ and $X_{\max }\left({ }^{16} \mathrm{O}\right)$ the high-density limit will be imposed by the value of $f$ that is required to furnish the correct $\alpha$-particle excess. Taking $X_{f}\left({ }^{16} \mathrm{O}\right)=0, \delta_{\alpha}=0.60$,

TABLE 5

\begin{tabular}{|c|c|c|c|c|c|c|}
\hline \multirow[t]{3}{*}{ MAXIMUM } & ${ }^{16} \mathrm{O}$ & $\begin{array}{l}\text { Mass FRA } \\
\text { CONSU }\end{array}$ & $\begin{array}{l}\text { NS FO } \\
\text { ION OF }\end{array}$ & $\delta_{\alpha}=0.6$ & AND & TotaI \\
\hline & & \multicolumn{5}{|c|}{$\Phi$} \\
\hline & & 0 & 1 & 2 & & $\infty$ \\
\hline$X_{\max }\left({ }^{16} \mathrm{O}\right)$ & & 0.64 & 0.74 & 0.81 & & 0.84 \\
\hline
\end{tabular}


TABLE 6

Values of $f$ to Give $\delta_{\alpha}=0.60$ for Various Initial ${ }^{16} \mathrm{O}$ Mass

FRACTIONS

\begin{tabular}{cccc}
\hline \hline \multicolumn{1}{c}{$X_{i}\left({ }^{16} \mathrm{O}\right)$} & $f$ & \multicolumn{1}{c}{$X_{\boldsymbol{i}}\left({ }^{16} \mathrm{O}\right)$} & \multicolumn{1}{c}{$f$} \\
\hline $0.80 \ldots \ldots \cdots \cdots$ & 0.998 & $0.40 \ldots \ldots \cdots \cdots$ & 0.727 \\
$0.70 \ldots \ldots \cdots \cdots$ & 0.960 & $0.30 \ldots \ldots \cdots \cdots$ & 0.546 \\
$0.60 \ldots \ldots \cdots \cdots$ & 0.908 & $0.20 \ldots \ldots \cdots \cdots$ & 0.184 \\
$0.50 \ldots \ldots \cdots \cdots$ & 0.935 & $0.17 \ldots \ldots \cdots \cdots$ & 0 \\
\hline
\end{tabular}

and $\phi=2.0$ in equation (39) we have the fraction of the ${ }^{16} \mathrm{O}$ which must burn by ${ }^{16} \mathrm{O}+{ }^{16} \mathrm{O}$ or ${ }^{12} \mathrm{C}+{ }^{16} \mathrm{O}$ as a function of the initial oxygen mass fraction given in table 6 .

\section{c) Abbreviated Network}

In order to examine in more analytic detail the interplay between $f$ and $\delta_{\alpha}$ and the allowed values of parameters, an abbreviated version of the generalized computerevolution program was developed. This program employed programming techniques designed for more speed and less generality and a greatly abbreviated reaction network. The network, shown in figure 12, represents quite well the coupling among the $\alpha$-nuclei $(Z=N=2 n, n$ integer) from carbon to calcium, as well as the coupling among the $\alpha$-plus-two-neutron species from silicon to argon. This latter feature is important because most of the neutron excess is maintained in these three species throughout the explosion. The abundances of these species can greatly influence the free-proton density and hence many important flows among other species. Reactions involving neutrons in either the entrance or exit channel were not considered in

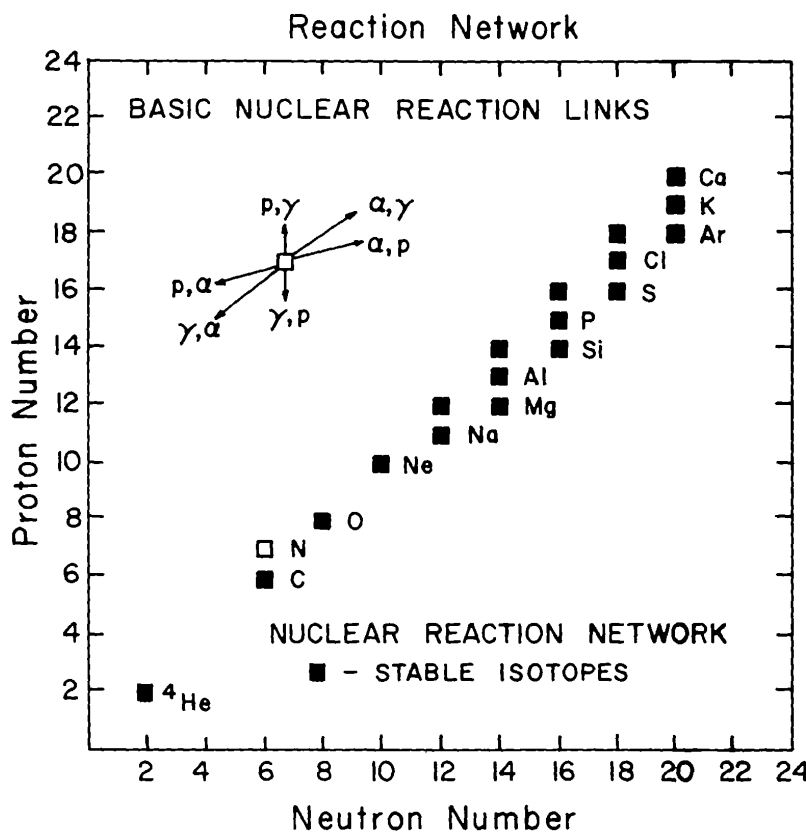

FIG. 12.-Abbreviated reaction network. This small network represents well only the coupling involving $\alpha$-particles and protons for key species lighter than calcium. Links due to the reactions $3 \alpha,{ }^{12} \mathrm{C}+{ }^{12} \mathrm{C},{ }^{12} \mathrm{C}+{ }^{16} \mathrm{O}$, and ${ }^{16} \mathrm{O}+{ }^{16} \mathrm{O}$ though not illustrated are also included. For peak temperatures $T_{9 i}<3.9$ this network yields results which approximate well the final mass fractions of the abundant elements between ${ }^{28} \mathrm{Si}$ and ${ }^{40} \mathrm{Ca}$ when compared to the results obtained with the much larger network shown in fig. $1 a$. 
the abbreviated program because we found them to be of much less importance than those involving $\alpha$-particles and protons in explosive oxygen burning. Modified branching ratios which adequately represent the usual behavior of the heavy nuclear reactions were employed. In the abbreviated network the effective products of the reaction ${ }^{12} \mathrm{C}+{ }^{12} \mathrm{C}$ are ${ }^{20} \mathrm{Ne}$ and an $\alpha$-particle, because in the large network most of the ${ }^{23} \mathrm{Na}$ (proton channel) and ${ }^{23} \mathrm{Mg}$ (neutron channel) produced by carbon burning disintegrate, to ${ }^{20} \mathrm{Ne}$ via ${ }^{23} \mathrm{Na}(p, \alpha)^{20} \mathrm{Ne},{ }^{23} \mathrm{Mg}(n, \alpha)^{20} \mathrm{Ne}$, and ${ }^{23} \mathrm{Mg}(n, p)^{23} \mathrm{Na}(p, \alpha)^{20} \mathrm{Ne}$. Similarly, the net product of the proton and neutron channels of the reaction ${ }^{12} \mathrm{C}+{ }^{16} \mathrm{O}$ is almost exclusively ${ }^{28} \mathrm{Si}$, since ${ }^{27} \mathrm{Al}$ and ${ }^{27} \mathrm{Si}$ are predominantly destroyed by the reactions ${ }^{27} \mathrm{Al}(p, \gamma)^{28} \mathrm{Si}$; and the neutron and deuteron channels of oxygen burning produce ${ }^{32} \mathrm{~S}$ since ${ }^{31} \mathrm{~S}$ and ${ }^{30} \mathrm{P}$ are destroyed by ${ }^{30} \mathrm{P}(\gamma, p){ }^{29} \mathrm{Si}(\alpha, n)^{32} \mathrm{~S}$ and ${ }^{31} \mathrm{~S}(\gamma, p){ }^{30} \mathrm{P}(\gamma, p){ }^{29} \mathrm{Si}(\alpha, n){ }^{32} \mathrm{~S}$. All other exit channels of these reactions were the same as in the large network. Such a simple network was found to be approximately 50 times faster than the usual 65-element network employed in previously described calculations in this paper. The representation of the final mass fractions of abundant species was excellent, and the value of $\delta_{\alpha}$ for the final abundance distribution of an evolution employing this simple code differed by not more than a few percent from the value of $\delta_{\alpha}$ obtained under the same circumstances using the generalized computer code with a 65 -element network. Of course this simple code cannot follow the breakthrough of matter into the iron peak and must therefore be inadequate for temperatures in excess of 3.9 billion degrees.

\section{d) Survey in $\rho_{i}, T_{9 i}$, and $X_{i}\left({ }^{16} \mathrm{O}\right)$}

We first undertook to determine those conditions of peak temperature and density which would yield $\delta_{\alpha}$ in the correct range for the reference composition given in equation (9): $X_{i}\left({ }^{16} \mathrm{O}\right)=0.54$ and $\phi=2.3$. The neutron excess was placed initially in ${ }^{30} \mathrm{Si}$ rather than ${ }^{26} \mathrm{Mg}$, however, which has the effect of lowering slightly the value of $\phi$ to 1.9 . We find that if we restrict ourselves to adiabatic expansion on a gravitational time scale $(\chi=1)$, the final distribution of elements coming out of this initial composition will agree closely with the solar values only if the peak temperature and density fall in the very restricted band shown in figure 13. Explosions of this nature that go to higher peak density (or lower temperature) will not produce enough excess $\alpha$-particles to satisfy the requirements for the production of the set $\left\{X_{f}\right\}_{\text {EOB }}$. In particular the simple code, backed up by sample calculations with the large code, shows ${ }^{40} \mathrm{Ca}$ to be underproduced by more than a factor of 2 in such regions. On the other hand if the density is lower (or temperature higher) than the allowed band, the final abundance of ${ }^{40} \mathrm{Ca}$ will be overproduced by more than a factor of 2 . Moreover, "alpha-rich" zones tend to produce sizable quantities of ${ }^{54} \mathrm{Fe}$ if the temperature is higher than $T_{9}=3.6$. This would make it difficult to get the right answer by averaging an "alpha-deficient" zone with an "alpha-rich" zone. Even if that were possible, the conditions shown in figure 13 should represent the conditions experienced by the "average zone" which produced the abundant species in the silicon to calcium region. For economy we will call this band of conditions resulting in acceptable abundances the "allowed region".

Also shown in figure 13 is the allowed region of peak conditions for a composition that is oxygen-rich $\left[X_{i}\left({ }^{16} \mathrm{O}\right)=0.80\right]$. This composition is very near to the "magic" value shown in table $5(\phi=2.0)$ and shows the characteristic unbounding of the peak density that we predicted earlier from simple arguments involving $f$ and $\delta_{\alpha}$. It is easy to see physically that this allowed region should be shifted to higher density in the $\left(\rho_{i}, T_{9 i}\right)$-plane than that for $X_{i}\left({ }^{16} \mathrm{O}\right)=0.54$. A larger initial oxygen mass fraction means that under identical conditions more oxygen will burn and produce more $\alpha$-particles. However, the alpha requirements of the composition are actually less since 


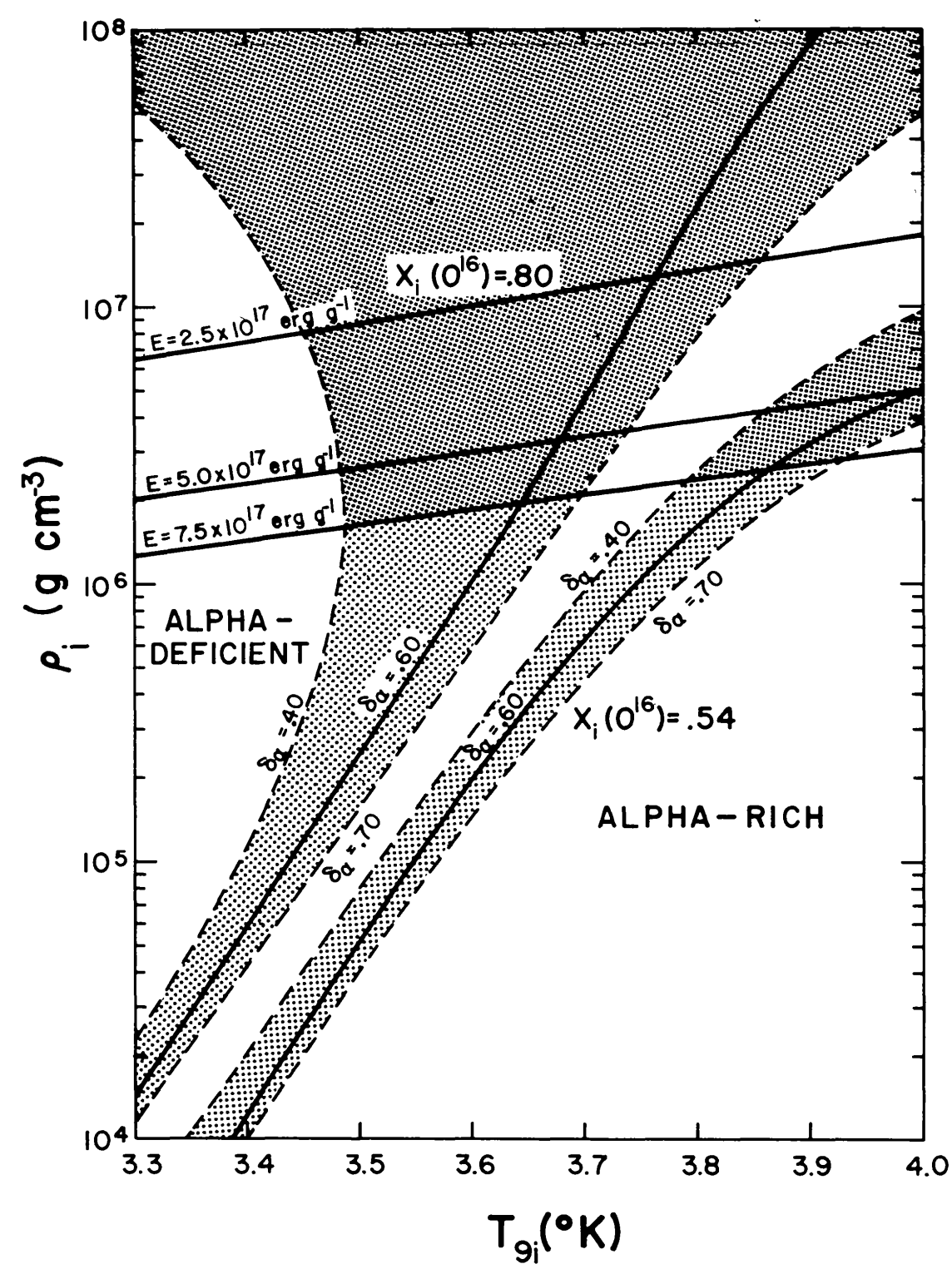

FIG. 13.-Allowed peak conditions for explosive oxygen burning. The values of peak density and temperature which result in the correct nucleosynthesis in explosive-oxygen-burning zones are shown for two different initial compositions. One contained 54 percent ${ }^{16} \mathrm{O}, 30$ percent ${ }^{24} \mathrm{Mg}$, 14 percent ${ }^{28} \mathrm{Si}$, and 2 percent ${ }^{26} \mathrm{Mg}$ by mass; the other contained 80 percent ${ }^{16} \mathrm{O}, 12$ percent ${ }^{24} \mathrm{Mg}$, 6 percent ${ }^{28} \mathrm{Si}$, and 2 percent ${ }^{26} \mathrm{Mg}$ by mass. The best abundance fit occurs along the lines $\delta_{\alpha}=0.60$. For regions to the left of the lines $\delta_{\alpha}=0.40$, the ratio ${ }^{40} \mathrm{Ca} /{ }^{28} \mathrm{Si}$ is mroe than a factor of 2 less than the solar value; for regions to the right of the lines $\delta_{\alpha}=0.70$, the same ratio is overproduced by more than a factor of 2 . Also shown are lines of various energy densities obtained from an appropriate equation of state (fig. 14). Points to the low-density side of the line $E=7.5 \times 10^{17}$ ergs $g^{-1}$ are energetically very difficult to attain.

the initial abundances of ${ }^{24} \mathrm{Mg}$ and ${ }^{28} \mathrm{Si}$ are smaller. Because increasing the density favors the less efficient alpha producing reactions ${ }^{12} \mathrm{C}+{ }^{16} \mathrm{O}$ and ${ }^{16} \mathrm{O}+{ }^{16} \mathrm{O}$ over the more efficient reactions ${ }^{16} \mathrm{O}(p, \alpha){ }^{13} \mathrm{~N}$ and ${ }^{16} \mathrm{O}(\gamma, \alpha){ }^{12} \mathrm{C}$, higher values of the peak density are allowable to compositions whose alpha requirements are smaller. In the case of the composition having $X_{i}\left({ }^{16} \mathrm{O}\right)=0.80$, the entire initial abundance of ${ }^{16} \mathrm{O}$ is, at high values of the initial density, being consumed by the reactions ${ }^{12} \mathrm{C}+{ }^{16} \mathrm{O}$ and ${ }^{16} \mathrm{O}+{ }^{16} \mathrm{O}$, leading to $f=1$. Under such conditions the total consumption at high 
density of $X_{i}\left({ }^{16} \mathrm{O}\right)=0.8$ leads finally to an acceptable abundance distribution $0.4<\delta_{\alpha}<0.6$ for a wide range of initial temperatures, as shown.

It is interesting to compare the regions suggested by nucleosynthesis arguments with restrictions that may be delineated by simple energetic considerations. In zones initially composed of carbon and oxygen, an upper bound to the energy available from nucleosynthesis should be approximately given by that energy liberated by the total conversion of all ${ }^{12} \mathrm{C}$ and ${ }^{16} \mathrm{O}$ present into iron-peak elements. This may be represented ${ }^{22} \mathrm{C}+2^{16} \mathrm{O} \rightarrow{ }^{56} \mathrm{Ni}+44.43 \mathrm{MeV}$ which yields $7.6 \times 10^{17} \mathrm{ergs}^{-1}$. This should be a rough limit to the energy density during a thermonuclear explosion. Employing an appropriate equation of state, the locus of the values of $\rho / \mu_{e}$ and $T_{9}$ that are specified by a given energy density in the neighborhood of the above value was determined. This equation of state contains contributions from electron pressure (relativistic and nonrelativistic, degenerate and nondegenerate), pair production, ion and photon pressure. Figure 14 shows these values for various energy densities. Points to the low-density side of the line $E=7.5 \times 10^{17} \mathrm{ergs}^{-1}$ should be energetically very difficult to attain. These energy-density lines are also shown in figure 13 and reveal an interesting fact. The overlap between the allowed region for $X_{i}\left({ }^{16} \mathrm{O}\right)=0.54$ and the area to the highdensity side of the line $E=7.5 \times 10^{17} \mathrm{ergs}^{-1}$ (densely shaded) is very small, while the overlap for the more oxygen-rich initial composition is quite large. This fact, along with the observation that the allowed region for $X_{i}\left({ }^{16} \mathrm{O}\right)=0.80$ is much larger regardless of any energetic considerations, leads us to the conclusion that it is much easier for an explosive-oxygen-burning zone to yield the correct nucleosynthesis if its initial composition contains a large amount of ${ }^{16} \mathrm{O}$, say $X_{i}\left({ }^{16} \mathrm{O}\right) \geqslant 0.60$, than it is if its initial composition is oxygen-deficient. If all our other assumptions and parameters were correct, this can be used to imply, on the basis of nucleosynthesis arguments

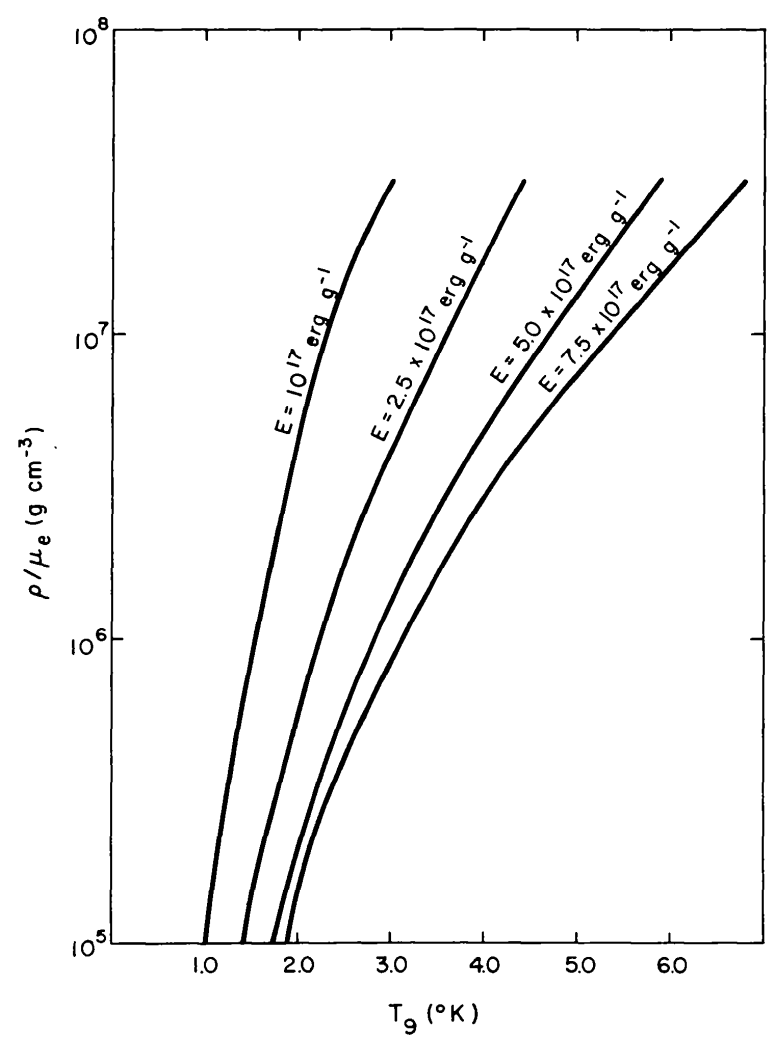

FIG. 14.-Values of $\rho / \mu_{e}$ and $T_{9}$ for various energy densities. Integration of the equation of state for the various energy densities shown in the figure is used to give $\rho$ as a function of $T_{9}$. 
alone, a lower mass limit to the original helium-core mass of the presupernova star. Recent computer evolutions of helium cores of various masses (Arnett 1972) have shown that the final abundance of ${ }^{12} \mathrm{C}$ after core helium burning in a helium core of mass $M_{\alpha}$ is approximately given by the empirical relationship

$$
X_{f}\left({ }^{12} \mathrm{C}\right)=0.60-\frac{2}{3} \log \zeta-0.267 \log \frac{M_{\alpha}}{M_{\odot}},
$$

where $\zeta=\theta_{\alpha}{ }^{2} / 0.85$ and $\theta_{\alpha}{ }^{2}$ is the reduced alpha width for the $7.12 \mathrm{MeV}$ state in the ${ }^{16} \mathrm{O}$ compound nucleus. The remainder of the composition is mainly ${ }^{16} \mathrm{O}$. Hydrostatic carbon burning does not greatly modify $X\left({ }^{16} \mathrm{O}\right)$ if allowance is made for ${ }^{20} \mathrm{Ne}$ photodisintegration (see Arnett and Truran 1969). Hence one would expect to arrive at the onset of oxygen burning with an oxygen fraction of approximately $X_{i}\left({ }^{16} \mathrm{O}\right) \simeq 1-$ $X_{f}\left({ }^{12} \mathrm{C}\right)$. Taking the currently accepted value $\zeta=1.0$, the condition $X_{i}\left({ }^{16} \mathrm{O}\right) \geqslant 0.60$ gives $M_{\alpha} \geqslant 10.0 M_{\odot}$. This result, though it should be regarded with caution and care paid to all the assumptions that entered its calculation, tends to indicate that our best candidate for a supernova which does explosive oxygen burning may very well be a massive star. We shall now examine the effect of varying some of the other parameters which enter into these calculations, namely the time scale and rates of critical nuclear reactions.

\section{e) Dependence on Time Scale}

The effect of varying the time scale which characterizes the explosive expansion has been examined by searching for the "allowed" region in the peak temperaturedensity plane for various values of the parameter $\chi$. The result is illustrated in figure 15 . An increased value of $\chi$ implies that for a given explosion the material remains at higher values of temperature and density (that is values near the peak values) for a longer period of time. Hence, (1) more oxygen is burned and (2) of the oxygen burned a larger fraction is burned by processes which are favored by higher temperature and/or density. Thus for larger $\chi$ we require nuclear evolutions at lower values of peak temperature and density. For diminished values of $\chi$ the effect is the opposite. The magnitude of the shift is illustrated for one particular initial composition in figure 15. Varying the expansion time scale an order of magnitude shifts the peak temperature scale of the allowed region about 0.4 billion degrees and the peak density scale about an order of magnitude from the values determined for $\chi=1.0$. Changing the time scale also influences slightly the peak value of temperature allowed in order not to produce excessive amounts of ${ }^{54} \mathrm{Fe}$ resulting in neutron deficiencies in the set $\left\{X_{f}\right\}_{\mathrm{EOB}}$. Decreasing $\chi$ a factor of 10 shifts the maximum temperature up from $T_{9}=3.9$ to about $T_{9}=4.05$. Increasing $\chi$ by a factor of 10 shifts the maximum temperature down to about $T_{9}=3.8$. Inspection of figure 15 shows that increasing the time scale gives a broader allowed range of peak densities for a given peak temperature. Thus, all other factors being equal, explosive oxygen burning on a time scale significantly longer than the "local hydrodynamic" time scale produces abundances which correlate better with the solar distribution for a wider range of peak conditions than burning on a shorter time scale.

\section{f) Dependence on Cross-Sections}

Certain key reaction rates can also significantly influence the boundaries of our nucleosynthesis-preferred region. These are the reactions that govern the rate of $\alpha$-particle production per ${ }^{28} \mathrm{Si}$ core. Of these, only the reactions ${ }^{16} \mathrm{O}+{ }^{16} \mathrm{O},{ }^{12} \mathrm{C}+{ }^{16} \mathrm{O}$, ${ }^{12} \mathrm{C}+{ }^{12} \mathrm{C},{ }^{16} \mathrm{O}(\gamma, \alpha){ }^{12} \mathrm{C}$, and ${ }^{16} \mathrm{O}(p, \alpha){ }^{13} \mathrm{~N}$ are important. Moreover, only the total reaction cross-sections for the first three reactions matter because $\delta_{\alpha}$ is independent of 


\section{EFFECT OF TIME SCALE $\left[X_{i}\left(0^{16}\right)=.70\right]$}

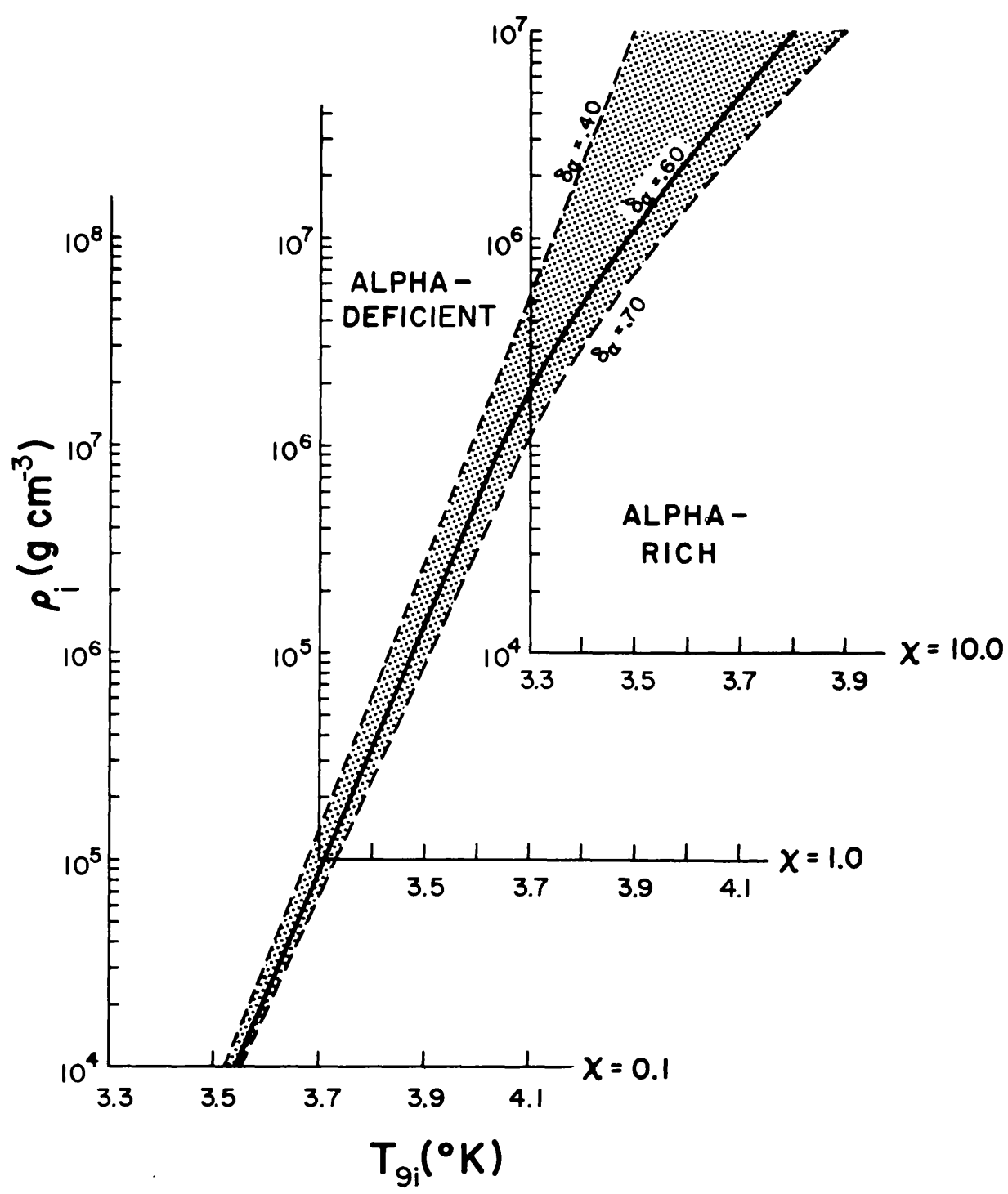

FIG. 15.-Effect of time scale. The effect of varying the expansion time scale by a factor of 10 in either direction about $\tau_{\mathrm{HD}}$ upon the required peak conditions for obtaining the correct abundance synthesis during explosive oxygen burning is presented for an initial composition of 70 percent ${ }^{16} \mathrm{O}, 18$ percent ${ }^{24} \mathrm{Mg}, 10$ percent ${ }^{28} \mathrm{Si}$, and 2 percent ${ }^{26} \mathrm{Mg}$ by mass. The effect of increasing the time scale an order of magnitude is roughly equivalent to decreasing the peak density required to obtain a given $\delta_{\alpha}$ by an order of magnitude and decreasing the peak temperature by about 0.4 in $T_{9}$. Longer time scales seem to give broader "allowed" regions.

the various branching ratios by decay from the compound nuclear states. We feel that the determinations of the rates from the published data for the ${ }^{16} \mathrm{O}+{ }^{16} \mathrm{O},{ }^{12} \mathrm{C}+{ }^{16} \mathrm{O}$, ${ }^{12} \mathrm{C}+{ }^{12} \mathrm{C}$, and ${ }^{16} \mathrm{O}(\gamma, \alpha){ }^{12} \mathrm{C}$ reactions are probably accurate to a factor of 2 in the energy range of interest. The values employed are given in the Appendix. The rate of the ${ }^{16} \mathrm{O}(p, \alpha)^{13} \mathrm{~N}$ reaction is adopted from Wagoner (1969) and may overestimate the actual rate by as much as an order of magnitude (Woosley et al. 1972a). We have undertaken to examine the sensitivity of our temperature and density restrictions to variations in these key cross-sections. The results are shown in table 7 which gives the 
TABLE 7

SHIFTS IN ALLOWABLE PEAK DENSITY RANGES IMPLIED BY VARIANT CROSS-SECTION

\begin{tabular}{|c|c|c|c|c|c|c|c|c|c|}
\hline \multirow{2}{*}{$\begin{array}{l}\text { Variant } \\
\text { Reaction }\end{array}$} & \multicolumn{3}{|c|}{$\mathrm{T}_{9 i}=3.4$} & \multicolumn{3}{|c|}{$\mathrm{T}_{9 \mathrm{i}}=3.6$} & \multicolumn{3}{|c|}{$\mathrm{T}_{9 i}=3.8$} \\
\hline & ${ }^{\delta}{ }_{\alpha}=0.7$ & $\delta_{\alpha}=0.6$ & ${ }^{s} a=0.4$ & ${ }^{\delta} \alpha=0.7$ & $\delta_{\alpha}=0.6$ & $\delta_{\alpha}=0.4$ & $\delta_{\alpha}=0.7$ & $\delta_{\alpha}=0.6$ & $\delta_{\alpha}=0.4$ \\
\hline All Normal & $2.5(4)$ & $3.2(4)$ & $5.0(4)$ & $3.1(5)$ & $4.2(5)$ & $1.2(6)$ & $3.2(6)$ & $4.6(6)$ & $4.0(7)$ \\
\hline$\left({ }^{16} 0+{ }^{16} 0\right) * 2$ & $2.5(4)$ & $3.2(4)$ & $5.0(4)$ & $3.0(5)$ & $4.2(5)$ & $1.6(6)$ & $2.5(6)$ & $3.9(6)$ & $3.5(7)$ \\
\hline$\left({ }^{16} 0+{ }^{16} 0\right) / 2$ & $2.5(4)$ & $3.2(4)$ & $5.0(4)$ & $3.1(5)$ & $4.2(5)$ & $1.0(6)$ & $3.6(6)$ & $5.0(6)$ & $3.2(7)$ \\
\hline$\left[{ }^{16} 0(\gamma, \alpha){ }^{12} \mathrm{C}\right] * 2$ & $7.0(4)$ & $8.9(4)$ & $1.4(5)$ & $8.0(5)$ & $1.2(6)$ & $3.6(6)$ & $5.4(6)$ & $7.9(6)$ & $6.0(7)$ \\
\hline$\left[{ }^{16} 0(\gamma, \alpha){ }^{12} \mathrm{c}\right] / 2$ & $9.4(3)$ & $1.2(4)$ & 1. $9(4)$ & $1.2(5)$ & $1.7(5)$ & $4.2(5)$ & $2.1(6)$ & $3.1(6)$ & $2.5(7)$ \\
\hline$\left(12 c+{ }^{16} 0\right) * 2$ & $1.8(4)$ & $2.3(4)$ & $3.6(4)$ & $1.7(5)$ & $2.4(5)$ & $6.3(5)$ & $1.6(6)$ & $2.3(6)$ & $1.6(7)$ \\
\hline$\left({ }^{12} \mathrm{c}+{ }^{16} 0\right) / 2$ & $3.2(4)$ & $4.0(4)$ & $6.5(4)$ & $5.0(5)$ & $7.3(5)$ & $2.3(6)$ & $5.3(6)$ & $7.9(6)$ & $7.9(7)$ \\
\hline$\left({ }^{12} c^{+}{ }^{12} c\right) \star 2$ & $2.6(4)$ & $3.3(4)$ & $5.4(4)$ & $3.9(5)$ & $5.6(5)$ & $1.6(6)$ & $4.1(6)$ & $6.1(6)$ & $5.0(7)$ \\
\hline$(12 c+12 c) / 2$ & $2.3(4)$ & $2.9(4)$ & $4.7(4)$ & $2.5(5)$ & $3.5(5)$ & $8.9(5)$ & $2.3(6)$ & $3.5(6)$ & $2.5(7)$ \\
\hline$\left[{ }^{16} \mathrm{o}(\mathrm{p}, \alpha)^{13} \mathrm{~N}\right\} / 2$ & $2.4(4)$ & $3.0(4)$ & $5.0(4)$ & $2.3(5)$ & $3.6(5)$ & $1.0(6)$ & $1.8(6)$ & $2.6(6)$ & $2.0(7)$ \\
\hline$\left[{ }^{16} \mathrm{O}(\mathrm{p}, \alpha)^{13} \mathrm{~N}\right] / 10$ & $2.3(4)$ & $2.9(4)$ & $4.9(4)$ & $1.8(5)$ & $3.0(5)$ & $9.0(5)$ & $6.3(5)$ & $1.3(6)$ & $1.0(7)$ \\
\hline
\end{tabular}

required values of peak density to produce distributions of characteristic $\delta_{\alpha}$ at several values of peak temperature when the five important cross-sections are varied. All calculations were carried out on a hydrodynamic time scale characterized by $\chi=1$ and for a composition of 70 percent ${ }^{16} \mathrm{O}, 20$ percent ${ }^{24} \mathrm{Mg}, 7$ percent ${ }^{28} \mathrm{Si}$, and 3 percent ${ }^{30} \mathrm{Si}$ by mass. For the most part the allowed region of temperatures and densities is fairly insensitive to variations of these reaction rates, most variations resulting in errors of a factor of 2 or less for the predicted density at a given temperature. Notable exceptions are the rates of the reactions ${ }^{16} \mathrm{O}(\gamma, \alpha){ }^{12} \mathrm{C}$ and ${ }^{16} \mathrm{O}(p, \alpha){ }^{13} \mathrm{~N}$ which, when varied within a range appropriate to their experimental determination, can give errors of up to a factor of 4 in the allowed density range. The effects of these cross-sections on the conditions may be understood in the light of how they affect the relative production of excess $\alpha$-particles and ${ }^{28} \mathrm{Si}$ cores. For instance, decreasing the ${ }^{16} \mathrm{O}+{ }^{16} \mathrm{O}$ rate means that we burn less oxygen. The less oxygen burned (for an initial composition containing sizable components of ${ }^{28} \mathrm{Si}$ and ${ }^{24} \mathrm{Mg}$ ) the more alpha-deficient the final distribution; i.e., the fewer $\alpha$-particles are produced per initial ${ }^{28} \mathrm{Si}$ and ${ }^{24} \mathrm{Mg}$. But with a reduced ${ }^{16} \mathrm{O}+{ }^{16} \mathrm{O}$ rate a larger fraction of the ${ }^{16} \mathrm{O}$ that is burned is consumed by reactions that tend to produce many $\alpha$-particles (that is $f$ decreases). In this fashion the decreased ${ }^{16} \mathrm{O}+{ }^{16} \mathrm{O}$ rate acts to produce more $\alpha$-particles. The two effects (decreased oxygen consumption and increased fractional contribution of efficient alpha-producing reactions) tend to cancel so that the net effect of varying the ${ }^{16} \mathrm{O}+{ }^{16} \mathrm{O}$ rate is to change the allowable density range very little. On the other hand, increasing the rate of the reaction ${ }^{16} \mathrm{O}(\gamma, \alpha)^{12} \mathrm{C}$ by a factor of 2 is seen to change the required density greatly. This is because (1) the increased rate means more ${ }^{16} \mathrm{O}$ is consumed thereby producing more $\alpha$-particles per initial ${ }^{28} \mathrm{Si}$ and ${ }^{24} \mathrm{Mg}$ and (2) a greater fraction of the oxygen is burned by a reaction that is very efficient at producing $\alpha$-particles ( $f$ is smaller). The two effects tend to add rather than cancel, thus giving a large shift. As usual, the direction of the shift is determined by the fact that high density favors reactions that make fewer $\alpha$-particles per ${ }^{28} \mathrm{Si}\left(\right.$ i.e., ${ }^{12} \mathrm{C}+{ }^{16} \mathrm{O}$ and 
${ }^{16} \mathrm{O}+{ }^{16} \mathrm{O}$ ) while low density favors ${ }^{16} \mathrm{O}(\gamma, \alpha){ }^{12} \mathrm{C}$ and ${ }^{16} \mathrm{O}(p, \alpha){ }^{13} \mathrm{~N}$. Thus to compensate for the greater abundance of excess $\alpha$-particles that can be produced by an increased ${ }^{16} \mathrm{O}(\gamma, \alpha){ }^{12} \mathrm{C}$ rate, the preferred density region is shifted to higher values. Similar considerations can explain the effects of the other critical reactions.

\section{g) Combined ${ }^{12} \mathrm{C}$ and ${ }^{16} \mathrm{O}$ Explosions}

Finally, we feel that this consideration of restrictions on explosive oxygen burning would not be complete without drawing to the readers attention an important fact regarding the initial composition requirements. Namely, the initial composition for explosive oxygen burning need not be the products of hydrostatic carbon burning. Indeed the initial composition may be a mixture of carbon and oxygen, i.e., the products of helium burning. All that really matters is that the peak temperature and density be appropriate to what we have determined for explosive oxygen burning. What happens is that in the early tiny fraction of a second the composition undergoes explosive carbon burning. The ${ }^{12} \mathrm{C}$ is all consumed before the temperature has fallen significantly, and a distribution of elements in the mass range $16 \leq A \leq 42$ is produced. All ${ }^{20} \mathrm{Ne}$ produced by the carbon burning is also transformed by photodisintegrationrearrangement reactions into ${ }^{16} \mathrm{O},{ }^{24} \mathrm{Mg},{ }^{28} \mathrm{Si}$, etc. before the temperature has begun to fall. Then the composition continues explosive oxygen burning and all proceeds as discussed previously. The only difference is that the "effective initial composition" is now much more alpha-rich than would have been obtained had the carbon been previously burned hydrostatically. Whereas the chief products of carbon bùrning followed by ${ }^{20} \mathrm{Ne}$ photodisintegration under hydrostatic conditions are ${ }^{16} \mathrm{O},{ }^{24} \mathrm{Mg}$, and ${ }^{28} \mathrm{Si}$, the burning under explosive conditions produces sizable quantities of ${ }^{32} \mathrm{~S}$, ${ }^{36} \mathrm{Ar}$, and ${ }^{40} \mathrm{Ca}$. The final ${ }^{16} \mathrm{O}$ abundance is also larger in the explosive case and the abundance of ${ }^{24} \mathrm{Mg}$ is smaller. All these factors combine to make an "effective initial composition" for explosive oxygen burning whose excess- $\alpha$-particle requirements are much less than would be obtained had the zone previously undergone hydrostatic carbon burning. The effect is analogous to what would be obtained by a larger initial ${ }^{16} \mathrm{O}$ mass fraction and a smaller fraction of ${ }^{24} \mathrm{Mg}$ and ${ }^{28} \mathrm{Si}$. In this case there will exist lower temperature and higher density solutions to the solar abundance problem than we have described for a star of a given core mass. Thus, the arguments giving the minimum helium core mass may not be valid if the zones in question have not previously undergone hydrostatic carbon burning. A preliminary investigation was done using the abbreviated network described previously in this section (in a zone of equal parts by mass of ${ }^{12} \mathrm{C}$ and ${ }^{16} \mathrm{O}$ plus three percent by mass of ${ }^{30} \mathrm{Si}$ for a neutron source). We find that explosive oxygen burning in this case can produce good agreement with the solar abundances of the set $\left\{X_{f}\right\}_{\mathrm{EOB}}$ for a wider range of temperatures and densities than in the previously described cases wherein the ${ }^{12} \mathrm{C}$ had burned prior to the explosion.

\section{SILICON BURNING}

If the peak temperature attained in explosive oxygen burning is sufficiently high or if the burning occurs on a time scale sufficiently long that the initial ${ }^{16} \mathrm{O}$ abundance is depleted before freeze-out, the qualitative behavior of the nuclear evolution will change. Instead of free particles and "seed ${ }^{28} \mathrm{Si}$ " being generated by oxygen burning, the main nuclear evolution is now characterized by "photodisintegration-rearrangement reactions" (see BCF), accompanying the photodisintegration of ${ }^{28} \mathrm{Si}$. The principal reactions involve the reduction of some part of the ${ }^{28} \mathrm{Si}$ to $\alpha$-particles which add onto the remaining ${ }^{28} \mathrm{Si}$ and other heavier species to gradually build nuclei of progressively higher $A$. Generally a sizable amount of iron-peak ( $50 \leqslant A \leqslant 62$ ) nuclei 
are produced. The overall process is popularly referred to as "silicon burning" and may be represented symbolically by

$$
2^{28} \mathrm{Si} \rightarrow{ }^{28} \mathrm{Si}+7 \alpha \rightarrow{ }^{56} \mathrm{Ni} .
$$

In silicon-burning ejecta the nuclear evolution has frozen out before the ${ }^{28} \mathrm{Si}$ has been exhausted. Complete silicon burning is characterized by the depletion of ${ }^{28} \mathrm{Si}$ before freeze-out occurs. This leads to a form of the $e$-process of Burbidge et al. (1957) and Fowler and Hoyle (1964). In the latter case it is found that most of the material has been processed into iron-peak nuclei and free particles. Different elements will be synthesized in the two cases.

Silicon burning has been described in considerable detail by BCF and Michaud and Fowler (1972) for the special case where quasi-equilibrium prevails in the $28 \leq A \leqslant 62$ region. For the high temperatures involved in silicon burning the assumption of quasiequilibrium is almost certainly a valid one throughout most of the evolution. However, the final abundances may be greatly modified during the relaxation from the high temperatures required in their calculation. Thus, though quite useful as a computational aid, quasi-equilibrium alone falls short in providing an adequate representation of the complete synthesis of the iron peak.

To depict silicon burning in its various phases it is necessary to employ the generalized computer code to evolve the coupled differential equations of flow. This attempt was first made by Truran et al. (1966). The network employed for our calculations, containing some 90 species, was shown in figure $1 b$. The large size of the network above iron was necessary to represent adequately the evolution during the freeze-out of the $e$-process which results in considerable production of species heavier than $A=56$. The network is primarily composed of species to the proton-rich side of the valley of beta-stability. This structure is due to the previous discovery, aided by use of quasiequilibrium surveys, that under silicon-burning conditions most of the species heavier than $A=40$ are not made as themselves but as proton-rich progenitors which subsequently decay to their stable isobar after freeze-out.

The network is somewhat sparse below $A=28$ because of the predominance of reactions which involve the alpha-nuclei ${ }^{12} \mathrm{C},{ }^{16} \mathrm{O},{ }^{20} \mathrm{Ne}$, and ${ }^{24} \mathrm{Mg}$. We offer some words of warning, however, about the effective rate at which ${ }^{28} \mathrm{Si}$ is burned explosively. At such high temperatures a few of the less tightly bound species may act as significant links between the alpha-nuclei. We have seen, for example, in explosive oxygen burning, how the reaction sequence ${ }^{16} \mathrm{O}(p, \alpha)^{13} \mathrm{~N}(\gamma, p){ }^{12} \mathrm{C}$ may act as an amplification factor on the ${ }^{16} \mathrm{O}(\gamma, \alpha)^{12} \mathrm{C}$ rate. The same thing happens during explosive silicon burning. The links $\left.{ }^{16} \mathrm{O}(p, \alpha)\right)^{13} \mathrm{~N}(\gamma, p){ }^{12} \mathrm{C}$ as well as ${ }^{16} \mathrm{O}(n, \alpha){ }^{13} \mathrm{C}(\gamma, n){ }^{12} \mathrm{C}$ may accelerate the rate of ${ }^{16} \mathrm{O}$ destruction to ${ }^{12} \mathrm{C}$, and the links ${ }^{24} \mathrm{Mg}(p, \alpha)^{21} \mathrm{Na}(\gamma, p)^{20} \mathrm{Ne}$ and ${ }^{24} \mathrm{Mg}(n, \alpha){ }^{21} \mathrm{Ne}(\gamma, n){ }^{20} \mathrm{Ne}$ may accelerate ${ }^{24} \mathrm{Mg}$ destruction to ${ }^{20} \mathrm{Ne}$. Under the conditions which we will find appropriate for explosive silicon burning these reactions could be very important. We left these reactions out of our silicon-burning calculations. We suspect that their calculated rates (Wagoner 1969) are too large, so that to include them might give a larger error to the burning rate than simply to omit them. The significance of these rates means that they warrant further laboratory study so that they can be properly included in future work. The reactions ${ }^{12} \mathrm{C}+{ }^{12} \mathrm{C},{ }^{12} \mathrm{C}+$ ${ }^{16} \mathrm{O}$, and ${ }^{16} \mathrm{O}+{ }^{16} \mathrm{O}$ can also play important roles in explosive silicon burning. By the recycling of material through ${ }^{24} \mathrm{Mg},{ }^{28} \mathrm{Si}$, etc. they can decrease the rate of silicon burning by up to a factor of 2 (if all material is recycled, i.e., no ${ }^{12} \mathrm{C}$ photodisintegrates). Because their rates are accurately known these reactions have been included in the network calculations, but since their effect is small, they have not been considered in the simple analytic formulae derived in $\S \mathrm{VI} d$. 


\section{a) Merging of Explosive Oxygen Burning into Silicon Burning}

Before discussing the nucleosynthesis from such evolutions though, it will first be of interest to examine the merging of explosive oxygen burning into silicon burning and the nature of the quasi-equilibrium which comes to be established as silicon burning progresses. For peak conditions sufficient to lead to the exhaustion of ${ }^{16} \mathrm{O}$ in explosive oxygen burning, the pair ${ }^{20} \mathrm{Ne}$ and ${ }^{16} \mathrm{O}$ should equilibrate with the free-alpha density before actual silicon burning commences as should the pair ${ }^{24} \mathrm{Mg}$ and ${ }^{28} \mathrm{Si}$. As long as a significant amount of ${ }^{16} \mathrm{O}$ remains unburned, the abundance of ${ }^{20} \mathrm{Ne}$ is large enough so that a small amount of $\alpha$-particle capture on ${ }^{20} \mathrm{Ne}$ is sufficient to dominate the photodisintegration flow downward from ${ }^{24} \mathrm{Mg}$ (after the initial abundance of ${ }^{24} \mathrm{Mg}$ is depleted). But once the ${ }^{16} \mathrm{O}$ abundance becomes too small to maintain the freeparticle densities required for strong flows, the time scale for nuclear reactions will increase. The small flow downwards from ${ }^{24} \mathrm{Mg}$ becomes important, and the diminishing abundance of ${ }^{20} \mathrm{Ne}$ is no longer sufficient to counteract it. Photodisintegration from ${ }^{24} \mathrm{Mg}$, whose small abundance is maintained by photodisintegration of ${ }^{28} \mathrm{Si}$, then becomes the dominant flow in the evolution and a new source of free particles. When this happens, in particular when the sum of the rates of ${ }^{20} \mathrm{Ne}(\alpha, \gamma)^{24} \mathrm{Mg}$ and ${ }^{20} \mathrm{Ne}(\alpha, p)$ ${ }^{23} \mathrm{Na}(p, \gamma)^{24} \mathrm{Mg}$ becomes less than the sum of the rates of the inverses of these reactions, silicon burning may be defined to have commenced. This reversal will generally occur when $X\left({ }^{16} \mathrm{O}\right) \leqslant 0.01$, the critical value being somewhat lower at lower temperatures. From that point on it is ${ }^{28} \mathrm{Si}$, not ${ }^{16} \mathrm{O}$, which dominates the burning.

The net rate of the photodisintegration flow downward from ${ }^{28} \mathrm{Si}$, and hence the rate of silicon burning, will depend in a critical fashion upon what species below ${ }^{28} \mathrm{Si}$ are in equilibrium with ${ }^{28} \mathrm{Si}$ and the free-alpha density. In particular, the net rate of destruction of ${ }^{28} \mathrm{Si}$ will be determined by the photodisintegration rate of the lightest species that is in equilibrium with ${ }^{28} \mathrm{Si}(\mathrm{BCF})$. If one considers silicon burning as an extension of oxygen burning, then ${ }^{24} \mathrm{Mg}$ is already in equilibrium with ${ }^{28} \mathrm{Si}$ at the onset. Similarly, ${ }^{20} \mathrm{Ne}$ is also in equilibrium with ${ }^{16} \mathrm{O}$. For low peak temperatures and short time scales then, it is the photodisintegration rate of ${ }^{24} \mathrm{Mg}$ which is important. For higher peak temperatures, usually for $T_{9 i} \geqslant 4.7,{ }^{20} \mathrm{Ne}$ may approach a state of equilibrium with ${ }^{24} \mathrm{Mg}$; then it is the rate of ${ }^{16} \mathrm{O}(\gamma, \alpha)^{12} \mathrm{C}$ which governs the flow downward from ${ }^{28} \overline{\mathrm{Si}}$. At temperatures sufficiently high to exhaust silicon, ${ }^{12} \mathrm{C}$ and even the free $\alpha$-particles may join in the equilibrium chain. The nuclei are then in true "nuclear statistical equilibrium," not just silicon quasi-equilibrium. This phenomena will be discussed at greater length later when the $e$-process is considered in detail.

During the early nuclear evolution the abundances of species lighter than ${ }^{28} \mathrm{Si}$ will depend on the initial composition. If the initial composition contains neither ${ }^{16} \mathrm{O}$ or ${ }^{24} \mathrm{Mg}$ then the small concentrations of these nuclei must first be established by the photodisintegration of heavier species before they can equilibrate. To illustrate these differences we compare in table 8 the $r_{j}$ values for several key species below ${ }^{28} \mathrm{Si}$ at several roughly equivalent times during the course of two calculations which employed the same initial thermodynamic conditions but different initial compositions. The initial thermodynamic conditions were $T_{9 i}=4.7$ and $\rho=2.0 \times 10^{7}$. Composition " "Si" was pure ${ }^{28} \mathrm{Si}$ with 3.0 percent of ${ }^{30} \mathrm{Si}$ for initial neutron enrichment. Composition " $O$ " was the standard explosive-oxygen-burning composition given in equation (9). It is apparent from the table that the explosive burning of composition " $\mathrm{O}$ " leads to the earlier establishment of small $r_{j}$ values for ${ }^{12} \mathrm{C},{ }^{16} \mathrm{O}$, and ${ }^{20} \mathrm{Ne}$ than the burning of composition " $\mathrm{Si}$ ". The abundance of ${ }^{24} \mathrm{Mg}$ very rapidly approaches its quasi-equilibrium value in either case. At the earliest time shown in table 8 the initial abundance of ${ }^{24} \mathrm{Mg}$ in composition "O" has not yet been depleted. (Recall that negative $r_{j}$ designates an overabundance.) The equilibrium structures of the two evolutions are virtually indistinguishable after $1.0 \times 10^{-3} \mathrm{~s}$ have elapsed. 
TABLE 8

VALUeS OF $r_{j}$ FOR SPECIES Lighter THAN ${ }^{28} \mathrm{Si}$

\begin{tabular}{|c|c|c|c|c|c|c|c|c|}
\hline & \multicolumn{8}{|c|}{ Composition Time } \\
\hline & $\begin{array}{c}\text { "O" } \\
5.2(-6)\end{array}$ & $\begin{array}{c}\text { "Si" } \\
6.1(-6)\end{array}$ & $\begin{array}{c}\text { “O”" } \\
2.2(-5)\end{array}$ & $\begin{array}{l}\text { "Si" } \\
2.9(-5)\end{array}$ & $\begin{array}{l}\text { "O" } \\
7.1(-5)\end{array}$ & $\begin{array}{l}\text { "Si" } \\
6.8(-5)\end{array}$ & $\begin{array}{l}\text { “O” } \\
4.9(-4)\end{array}$ & $\begin{array}{l}\text { "Si" } \\
5.6(-4)\end{array}$ \\
\hline $\begin{array}{c}\text { Key Abundances: } \\
X\left({ }^{28} \mathrm{Si}\right) \ldots \ldots \\
X\left({ }^{16} \mathrm{O}\right) \ldots \ldots \\
X(\alpha) \ldots \ldots \\
\text { Key } r, \text { Values: }\end{array}$ & $\begin{array}{l}+0.706 \\
+0.192 \\
+2.57(-4)\end{array}$ & $\begin{array}{l}+0.913 \\
+0.002 \\
+1.10(-4)\end{array}$ & $\begin{array}{l}+0.735 \\
+0.055 \\
+7.96(-4)\end{array}$ & $\begin{array}{l}+0.895 \\
+0.005 \\
+2.00(-4)\end{array}$ & $\begin{array}{l}+0.735 \\
+0.013 \\
+9.64(-4)\end{array}$ & $\begin{array}{l}+0.866 \\
+0.006 \\
+3.42(-4)\end{array}$ & $\begin{array}{l}+0.710 \\
+0.002 \\
+1.10(-3)\end{array}$ & $\begin{array}{l}+0.751 \\
+0.002 \\
+8.95(-4)\end{array}$ \\
\hline $\begin{array}{l}\text { Key } r_{j} \text { Values: } \\
r\left({ }^{32} \mathrm{~S}\right) \\
r\left({ }^{24} \mathrm{Mg}\right) \\
r\left({ }^{20} \mathrm{Ne}\right) \\
r\left({ }^{16} \mathrm{O}\right) \\
r\left({ }^{12} \mathrm{C}\right)\end{array} \ldots .$. & $\begin{array}{l}+0.048 \\
-0.41 \\
+0.29 \\
+0.57 \\
+3.79\end{array}$ & $\begin{array}{l}+0.008 \\
-0.005 \\
+1.88 \\
+3.87 \\
+6.55\end{array}$ & $\begin{array}{l}+0.006 \\
-0.035 \\
-0.42 \\
-0.35 \\
+2.06\end{array}$ & $\begin{array}{l}+0.005 \\
-0.004 \\
+1.51 \\
+2.54 \\
+4.95\end{array}$ & $\begin{array}{l}+0.002 \\
-0.009 \\
-0.081 \\
+0.013 \\
+1.98\end{array}$ & $\begin{array}{l}+0.004 \\
-0.003 \\
+1.14 \\
+1.76 \\
+3.98\end{array}$ & $\begin{array}{l}+0.003 \\
-0.004 \\
+0.47 \\
+0.70 \\
+2.15\end{array}$ & $\begin{array}{l}+0.002 \\
-0.003 \\
+0.58 \\
+0.85 \\
+2.45\end{array}$ \\
\hline
\end{tabular}




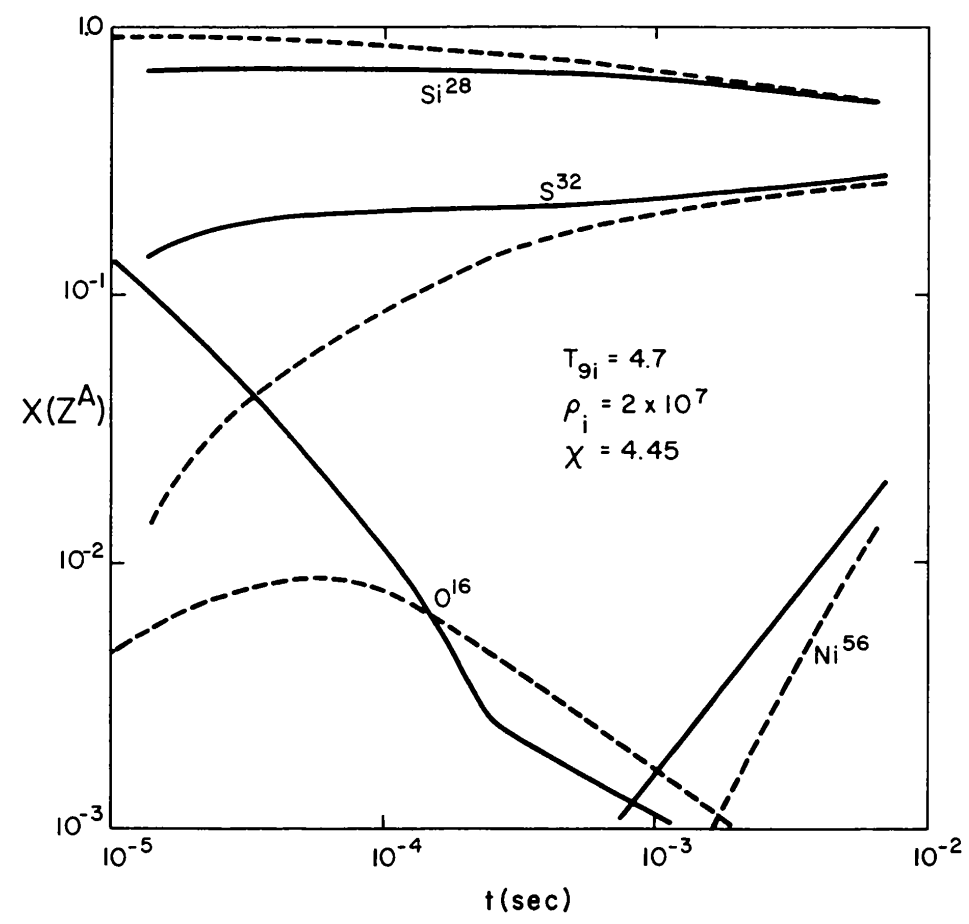

FIG. 16.-Effect of different initial compositions upon silicon burning. The mass fractions of four key species are shown as a function of the elapsed time for two runs employing identical thermodynamic conditions but different initial compositions. The solid lines are obtained if the initial composition is the usual one employed in oxygen-burning calculations (composition " $\mathrm{O}$ "). Dashed lines result from the burning of composition " $\mathrm{Si}$ " consisting of 97 percent by mass of ${ }^{28} \mathrm{Si}$ and 3.0 percent by mass of ${ }^{30} \mathrm{Si}$. The evolutions became virtually indistinguishable after the elapsed time became greater than $0.01 \mathrm{~s}$.

Not only the equilibrium character but also the resultant composition is somewhat different for composition "O," at least in the early stages. A good deal of material has already been processed into species heavier than ${ }^{28} \mathrm{Si}$ by oxygen burning in composition " $\mathrm{O}$ " before silicon burning begins. Thus, after equal elapsed times, burning of composition " $\mathrm{O}$ " results in more heavy species than burning pure silicon. As time passes, however, the results of the two compositions become more similar as the effect of the "head start" given by oxygen burning becomes less important. This effect is illustrated in figure 16. Once the silicon mass fraction in the "pure silicon-burning run" drops below about 60 percent, the evolution is basically identical to an extended oxygen-burning run under the same conditions. A similar type of behavior has been found by Truran (1968a) to occur at lower temperatures. Figure 16 here warrants comparison to his figure 4.

\section{b) The Approach to Quasi-Equilibrium}

It is now of considerable interest to examine again the equilibrium state of species heavier than ${ }^{28} \mathrm{Si}$, for the two runs defined in table 8. Table 9 shows the values of $r_{j}$ for species having $A \geq 28$ at three times during the same two runs. The table reveals the existence of two large quasi-equilibrium clusters, one containing species having $28 \leq A \leqslant 45$ and the other having species with $A \geqslant 45$. The many clusters observed previously in explosive oxygen burning have merged to the point that only these two, separated by a barrier in the vicinity of calcium, still remain. The nuclei in the barrier are identifiable by transitional values of $r_{j}$. The reason for the barrier is the low quasi-equilibrium abundances in this region (BCF, $\S \mathrm{VII})$. It is of interest to note that 
TABLE 9

VALUES OF $r_{j}$ FOR KEY SPECIES HEAVIER THAN $\mathrm{si}^{28}$ DURING EARLY STAGES OF SILICON BURNING $\mathrm{T}_{9 i}=4.7, \rho_{i}=2 \times 10^{7}$

\begin{tabular}{|c|c|c|c|c|c|c|}
\hline \multicolumn{3}{|c|}{$t=1.4 \times 10^{-5} \mathrm{sec}$} & \multicolumn{2}{|c|}{$t=1.1 \times 10^{-4} \mathrm{sec}$} & \multicolumn{2}{|c|}{$t=1.1 \times 10^{-3} \mathrm{sec}$} \\
\hline Species & Composition "Si" & Composition "0" & Composition "Si" & Composition "0" & Composition "Si" & Composition ' $\mathrm{b}$ ' \\
\hline${ }^{28} \mathrm{si}$ & 0 & 0 & 0 & 0 & 0 & 0 \\
\hline $32 \mathrm{~s}$ & .006 & .014 & .003 & .002 & .003 & .003 \\
\hline${ }^{36} \mathrm{Ar}$ & .014 & .024 & .006 & .004 & .005 & .005 \\
\hline${ }^{38} \mathrm{Ar}$ & .045 & $: 057$ & .022 & .021 & .022 & .023 \\
\hline${ }^{40} \mathrm{Ca}$ & .038 & .044 & .014 & .013 & .014 & .014 \\
\hline${ }^{41} \mathrm{Ca}$ & .045 & .051 & .020 & .016 & .015 & .015 \\
\hline $42 \mathrm{Ca}$ & .053 & .073 & .028 & .030 & .027 & .028 \\
\hline${ }^{43} \mathrm{sc}$ & .056 & .075 & .032 & .030 & .028 & .029 \\
\hline${ }^{44} \mathrm{Sc}$ & .079 & .108 & .060 & .048 & .033 & .032 \\
\hline${ }^{45} \mathrm{Sc}$ & .278 & .580 & .361 & .332 & .082 & .064 \\
\hline${ }^{46} \mathrm{Sc}$ & .359 & .930 & .496 & .527 & .114 & .093 \\
\hline${ }^{44} \mathrm{Ti}$ & .053 & .063 & .032 & .021 & .015 & .015 \\
\hline${ }^{45} \mathrm{Ti}$ & .248 & .490 & .317 & .271 & .057 & .041 \\
\hline${ }^{46} \mathrm{Ti}$ & .467 & 1.09 & .562 & .550 & .098 & .075 \\
\hline${ }^{47} \mathrm{Ti}$ & .504 & 1.23 & .601 & .580 & .100 & .077 \\
\hline${ }^{47} \mathrm{v}$ & .473 & 1.10 & .566 & .552 & .098 & .075 \\
\hline $48 \mathrm{v}$ & .518 & 1.28 & .615 & .588 & .100 & .077 \\
\hline${ }^{49} \mathrm{v}$ & .658 & 1.69 & .718 & .617 & .101 & .077 \\
\hline${ }^{48} \mathrm{Cr}$ & .478 & 1.13 & .570 & .545 & .082 & .060 \\
\hline${ }^{49} \mathrm{Cr}$ & .622 & 1.56 & .685 & .595 & .085 & .061 \\
\hline${ }^{50} \mathrm{Cr}$ & .688 & 1.74 & .712 & .606 & .084 & .060 \\
\hline${ }^{51} \mathrm{Mn}$ & .696 & 1.75 & .714 & .607 & .084 & .060 \\
\hline${ }^{52} \mathrm{Fe}$ & .681 & 1.72 & .696 & .592 & .069 & .046 \\
\hline${ }^{53} \mathrm{Fe}$ & .779 & 1.97 & .730 & .600 & .067 & .044 \\
\hline${ }^{54} \mathrm{Fe}$ & .795 & 2.02 & .736 & .602 & .067 & .043 \\
\hline${ }^{55} \mathrm{Co}$ & .800 & 2.02 & .737 & .604 & .068 & .044 \\
\hline${ }^{56} \mathrm{Ni}$ & .781 & 1.99 & .718 & .589 & .053 & .030 \\
\hline${ }^{57} \mathrm{Ni}$ & .797 & 2.02 & .721 & .588 & .035 & .028 \\
\hline${ }^{58 \mathrm{Ni}}$ & .802 & 2.02 & .721 & .587 & .040 & .027 \\
\hline${ }^{59} \mathrm{Cu}$ & .806 & 2.02 & .722 & .588 & .050 & .028 \\
\hline${ }^{60} \mathrm{zn}$ & .792 & 1.99 & .708 & .576 & .038 & .016 \\
\hline
\end{tabular}

the evolution employing composition " $\mathrm{Si}$ " is somewhat closer to attaining overall quasi-equilibrium in the $28 \leq A \leq 66$ range at $t=1.4 \times 10^{-5}$ s than the similar oxygen-burning evolution. This is due to the presence of unburned oxygen at this time in the oxygen-burning run. Since oxygen burning produces $\alpha$-particles more rapidly than the burning of pure silicon under the same conditions, the composition of the oxygen-burning run is much more alpha-rich at this point. In particular, $X_{\alpha}$ is about eight times larger and so, therefore, is the ratio $X\left({ }^{32} \mathrm{~S}\right) / X\left({ }^{28} \mathrm{Si}\right)$. Since incomplete silicon quasi-equilibrium has abundances of species in the iron peak proportional to approximately the seventh power of $X_{\alpha}$, more material must be pushed through the barrier near $A=45$ to bring the iron peak into equilibrium with ${ }^{28} \mathrm{Si}$. As a result, at this point the oxygen-burning evolution is lagging behind in the establishment of overall quasi-equilibrium. Shortly thereafter, with more than 80 percent of the ${ }^{28} \mathrm{Si}$ remaining $\left(t=1.1 \times 10^{-4} \mathrm{~s}\right)$ quasi-equilibrium has been established to within a factor of 5 for all species above ${ }^{28} \mathrm{Si}$ in both runs. At the later time $t=1.1 \times 10^{-3} \mathrm{~s}$, all species above ${ }^{28} \mathrm{Si}$ are in quasi-equilibrium to within 25 percent for both runs. From that point on, quasi-equilibrium is a good approximation until freeze-out effects 
set in and the distribution begins to refragment into clusters. For these runs this occurs when the temperature has fallen to $T_{9} \leqslant 3.4$.

The net flows leading to the establishment of the final consolidation of the iron-peak quasi-equilibrium cluster and the ${ }^{28} \mathrm{Si}$ quasi-equilibrium cluster are shown for the oxygen-burning run after an elapsed time $t=1.4 \times 10^{-5} \mathrm{~s}$ in figure 17. The principal forward flow joining the two groups is ${ }^{45} \mathrm{Sc}(p, \gamma)^{46} \mathrm{Ti}$. Examination of a wide variety of extended oxygen-and-silicon-burning runs at temperatures high enough to explosively burn nonnegligible amounts of silicon shows that ${ }^{45} \mathrm{Sc}(p, \gamma)^{46} \mathrm{Ti}$ is always the dominant, or at least one of the dominant, flows linking the two groups for nontrivial $\eta$ (see e.g., Truran $1968 b$, figs. $4 a$ and $b$ ). At higher temperatures ${ }^{42} \mathrm{Ca}(\alpha, \gamma)^{46} \mathrm{Ti}$ and ${ }^{45} \mathrm{Ti}(n, \gamma){ }^{46} \mathrm{Ti}$ may contribute somewhat to the breakthrough but never completely overshadow ${ }^{45} \operatorname{Sc}(p, \gamma)^{46} \mathrm{Ti}$. Furthermore, the chief reverse flow from the iron-peak quasi-equilibrium group is usually ${ }^{46} \mathrm{Ti}(\gamma, p)^{45} \mathrm{Sc}$.

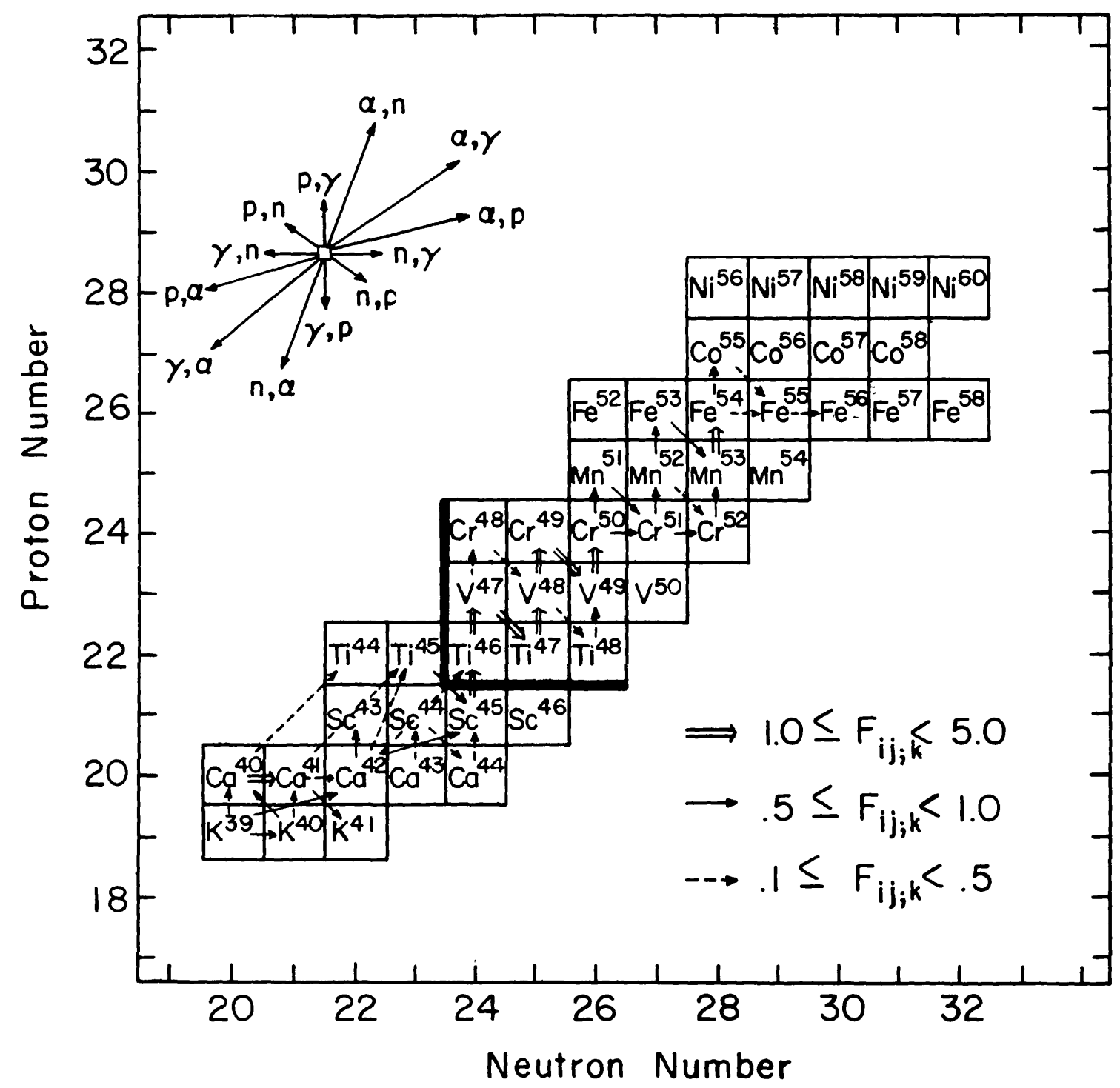

FIg. 17.-Dominant flows leading to quasi-equilibrium during silicon burning. Strong nuclear flows of the type and strength specified in the figure are shown at a time of $1.4 \times 10^{-5} \mathrm{~s}$ into the burning of composition " $O$." The initial temperature and density were $T_{9}=4.7$ and $\rho=2.0 \times$ $10^{7}$, respectively. The time-scale factor was $\chi=4.45$, and the initial value of $\eta$ was 0.002 . 
The dominance of ${ }^{45} \mathrm{Sc}(p, \gamma)^{46} \mathrm{Ti}$ on the transfer of nuclei from the low- $A$ cluster into the high- $A$ cluster warrants some explanation, because BCF and Michaud and Fowler (1972) both placed emphasis on ${ }^{44} \mathrm{Ti}(\alpha, p)^{47} \mathrm{~V}$ as a reaction bridging the bottleneck. Their considerations were based on the high quasi-equilibrium abundance of ${ }^{44} \mathrm{Ti}$ at a time when much of the silicon has already been converted to ${ }^{56} \mathrm{Ni}$. That conclusion is correct, but it is correct at a time long after quasi-equilibrium is first established. The relevant question is that of which reactions transfer nuclei through the bottleneck earlier in the computation when the high- $A$ cluster is still underabundant relative to quasi-equilibrium expectations. At these earlier times the abundance of ${ }^{45} \mathrm{Sc}$ is comparable to that of ${ }^{44} \mathrm{Ti}$ in the high-temperature explosions we are considering, and the abundance of ${ }^{46} \mathrm{Ti}$ greatly exceeds that of ${ }^{44} \mathrm{Ti}$. It is because of these features that proton reactions with ${ }^{45} \mathrm{Sc}$ greatly exceed $\alpha$-particle reactions with ${ }^{44} \mathrm{Ti}$. Only much later in the burning, when quasi-equilibrium is already a good approximation, does ${ }^{44} \mathrm{Ti}(\alpha, p)^{47} \mathrm{~V}$ become important. Its importance is also increased by the lower temperatures considered in the two papers cited above. Since we are interested here in the time required for the high- $A$ cluster to achieve quasi-equilibrium with the low- $A$ cluster, it happens that considering only the reaction ${ }^{45} \operatorname{Sc}(p, \gamma){ }^{46} \mathrm{Ti}$ is more than adequate; in fact, for the evolutions of table 8 , it makes an error of about 25 percent, which is due primarily to the ${ }^{42} \mathrm{Ca}(\alpha, \gamma){ }^{46} \mathrm{Ti}$ and ${ }^{45} \mathrm{Ti}(n, \gamma){ }^{46} \mathrm{Ti}$ reactions. Thus the differential equations connecting the two groups may be approximately represented by

$$
\begin{gathered}
\frac{d G_{1}}{d t}=-Y\left({ }^{45} \mathrm{Sc}\right) Y_{p} \lambda_{p y}\left({ }^{45} \mathrm{Sc}\right)+Y\left({ }^{46} \mathrm{Ti}\right) \lambda_{y p}\left({ }^{46} \mathrm{Ti}\right), \\
\frac{d G_{2}}{d t}=Y\left({ }^{45} \mathrm{Sc}\right) Y_{p} \lambda_{p \gamma}\left({ }^{45} \mathrm{Sc}\right)-Y\left({ }^{46} \mathrm{Ti}\right) \lambda_{\gamma p}\left({ }^{46} \mathrm{Ti}\right) \\
G_{1}=\sum_{i=28}^{45} Y(i), \quad G_{2}=\sum_{i=46}^{66} Y(i)
\end{gathered}
$$

where $G_{1}$ and $G_{2}$ are the sums of the number densities (divided by $\rho N_{\mathrm{A}}$ ) of nuclei in the silicon-based and iron-peak-based quasi-equilibrium groups, respectively. Define $f_{1}^{45}=\left\langle Y\left({ }^{45} \mathrm{Sc}\right) / G_{1}\right\rangle$ and $f_{2}^{46}=\left\langle Y\left({ }^{46} \mathrm{Ti}\right) / G_{2}\right\rangle$ where the brackets denote an average of the quantity over the time when equilibration is occurring. These quantities are clearly the average fractional abundances of ${ }^{45} \mathrm{Sc}$ and ${ }^{46} \mathrm{Ti}$ in groups $G_{1}$ and $G_{2}$, respectively. The above equations may then be written in the suggestive form

$$
\begin{aligned}
& \frac{d G_{1}}{d t}=-f_{1}{ }^{45} G_{1} Y_{p} \lambda_{p y}\left({ }^{45} \mathrm{Sc}\right)+f_{2}{ }^{46} G_{2} \lambda_{y p}\left({ }^{46} \mathrm{Ti}\right), \\
& \frac{d G_{2}}{d t}=f_{1}^{45} G_{1} Y_{p} \lambda_{p \gamma}\left({ }^{45} \mathrm{Sc}\right)-f_{2}^{46} G_{2} \lambda_{y p}\left({ }^{46} \mathrm{Ti}\right) .
\end{aligned}
$$

To good approximation we can regard all quantities except $G_{1}$ and $G_{2}$ as constants, so that the coupled set of differential equations has the solution

$$
\frac{G_{2}}{G_{1}}=\frac{\left(r_{2} / r_{1}\right) A \exp \left[\left(r_{1}+r_{2}\right) t\right]+1}{A \exp \left[\left(r_{1}+r_{2}\right) t\right]-1},
$$

where

$$
\begin{gathered}
A \equiv \frac{\left(G_{1} / G_{2}\right)_{i}+1}{\left(G_{1} / G_{2}\right)_{i}-\left(r_{2} / r_{1}\right)}, \\
\left.r_{1} \equiv f_{1}{ }^{45} Y_{p} \lambda_{p \gamma}{ }^{45} \mathrm{Sc}\right), \quad r_{2} \equiv f_{2}{ }^{46} \lambda_{\gamma p}\left({ }^{46} \mathrm{Ti}\right),
\end{gathered}
$$


and where $\left(G_{1} / G_{2}\right)_{i}$ is the initial value of the ratio $G_{1} / G_{2}$. We find numerically that during the time quasi-equilibrium is being established one generally has $G_{1} \gg G_{2}$ and $G_{1} r_{1} \gg G_{2} r_{2}$. Then $A \simeq 1$ and $G_{1}$ and $G_{2}$ should approach their quasi-equilibrium value

$$
\left(\frac{G_{1}}{G_{2}}\right)_{\mathrm{de}}=\frac{r_{2}}{r_{1}}
$$

in a time $t \geqslant\left(r_{2}\right)^{-1}$. The time involves $r_{2}$ because it is usually larger than $r_{1}$ due to the small value of $f_{1}{ }^{45}$. Hence a fairly accurate lower bound to the time scale for equilibration between $G_{1}$ and $G_{2}$ is

$$
\tau_{\min }=\left[f_{2}{ }^{46} \lambda_{\gamma p}\left({ }^{46} \mathrm{Ti}\right)\right]^{-1} \text {. }
$$

Values of $f_{2}{ }^{46}$ and $\tau_{\min }$ are given for 10 runs in table 10 . The values $f_{2}{ }^{46}$ and $\tau_{\min }$ are evaluated at the time when total quasi-equilibrium for $A \geq 28$ has been achieved to within a factor of 2 . The elapsed time, $t_{2}$, in the given run at that point is also given. It is seen that the agreement of $t_{2}$ and $\tau_{\min }$ is good to within an order of magnitude. The quantity $\tau_{\min }$ is always a lower bound on $t_{2}$ as one would expect. Here it seems that $t_{2}=4 \tau_{\min }$ is a good approximation. Moreover the fraction of ${ }^{46} \mathrm{Ti}, f_{2}{ }^{46}$, seems to be a reasonably well behaved function of the temperature only. This result is not too surprising since the elements in $G_{2}$ are in approximate quasi-equilibrium with ${ }^{46} \mathrm{Ti}$. Table 10 shows that the ${ }^{28} \mathrm{Si}$ mass fraction is roughly constant at the point of evaluation of $f_{2}{ }^{46}$. Since $X\left({ }^{28} \mathrm{Si}\right), \rho, \eta$, and $T_{9}$ determine a unique quasi-equilibrium in $G_{1}$ (since $G_{1} \gg G_{2}$ ) and hence unique free-particle densities, this implies a unique quasiequilibrium relative to ${ }^{46} \mathrm{Ti}$ in $G_{2}$. A quasi-equilibrium distribution varies little with density (compared with its dependence on the temperature); therefore, fixing $\eta$ and $X\left({ }^{28} \mathrm{Si}\right)$ makes $f_{2}{ }^{46}$ an approximate function of $T_{9}$. Empirically it is determined that in the region $T_{9}=4.7$ to $T_{9}=8.3$

$$
f_{2}^{46} \simeq 39 e^{-39 / T_{9}}
$$

to within a factor of 2 for the ten points in table 10 (correlation coefficient $=0.94$ ). We obtain a good approximation to the time required for approaching quasi-equilibrium by inserting equation (48) into equation (47) and by multiplying by the semiempirical factor of 4 converting $\tau_{\min }$ to $t_{2}$,

$$
\tau_{\mathrm{qe}} \simeq 1.8 \times 10^{-20} e^{176.3 / T_{9}} .
$$

\begin{tabular}{|c|c|c|c|c|c|c|c|c|}
\hline $\begin{array}{l}\text { Com- } \\
\text { position }\end{array}$ & $T_{9 i}$ & $\rho_{i}$ & $f_{2}^{48}$ & $39 e^{-39 / T_{0 i}}$ & $X\left({ }^{28} \mathrm{Si}\right)$ & $t_{2}$ & $\tau_{\min }$ & $\tau_{\mathrm{qe}}$ \\
\hline 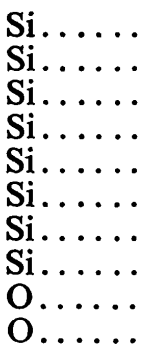 & $\begin{array}{l}4.7 \\
5.0 \\
5.5 \\
5.5 \\
5.9 \\
6.68 \\
8.1 \\
8.3 \\
4.7 \\
5.5\end{array}$ & $\begin{array}{r}2 \times 10^{7} \\
2 \times 10^{7} \\
5 \times 10^{5} \\
2 \times 10^{7} \\
2 \times 10^{8} \\
3.2 \times 10^{7} \\
6.2 \times 10^{8} \\
1.7 \times 10^{9} \\
2 \times 10^{7} \\
2 \times 10^{7}\end{array}$ & $\begin{array}{l}0.0084 \\
0.010 \\
0.070 \\
0.026 \\
0.095 \\
0.16 \\
0.18 \\
0.21 \\
0.0035 \\
0.016\end{array}$ & $\begin{array}{l}0.0097 \\
0.016 \\
0.032 \\
0.032 \\
0.053 \\
0.12 \\
0.32 \\
0.35 \\
0.0097 \\
0.032\end{array}$ & $\begin{array}{l}0.77 \\
0.74 \\
0.63 \\
0.78 \\
0.62 \\
0.66 \\
0.58 \\
0.63 \\
0.69 \\
0.67\end{array}$ & $\begin{array}{l}4 \times 10^{-4} \\
5 \times 10^{-5} \\
1 \times 10^{-6} \\
2 \times 10^{-6} \\
1 \times 10^{-7} \\
4 \times 10^{-9} \\
6 \times 10^{-11} \\
4 \times 10^{-11} \\
2 \times 10^{-4} \\
2 \times 10^{-6}\end{array}$ & $\begin{array}{l}1 \times 10^{-4} \\
1 \times 10^{-5} \\
2 \times 10^{-7} \\
5 \times 10^{-7} \\
2 \times 10^{-8} \\
9 \times 10^{-10} \\
2 \times 10^{-11} \\
1 \times 10^{-11} \\
2 \times 10^{-4} \\
8 \times 10^{-7}\end{array}$ & $\begin{array}{l}4 \times 10^{-4} \\
4 \times 10^{-5} \\
2 \times 10^{-6} \\
2 \times 10^{-6} \\
2 \times 10^{-7} \\
5 \times 10^{-9} \\
5 \times 10^{-11} \\
3 \times 10^{-11} \\
4 \times 10^{-4} \\
2 \times 10^{-6}\end{array}$ \\
\hline
\end{tabular}

TABLE 10

Time Scales in Seconds for Attaining Quasi-Equilibrium during Silicon Burning 
Table 10 shows that this result does closely agree with $t_{2}$. In an explosion the quasiequilibrium can be achieved if the required time is less than about $0.1 \tau_{\text {HD }}$, which is a reasonable characterization of the duration of extensive nuclear burning. Straightforward evaluation of this inequality yields

$$
T_{9 i}>\frac{176.3}{40.0+\ln \left[\chi\left(\rho / 10^{8}\right)^{-1 / 2}\right]} \cdot
$$

Expansions having initial temperatures in excess of this value will achieve quasiequilibrium between $G_{1}(28 \leq A \leq 45)$ and $G_{2}(46 \leq A \leq 66)$ at some time during the expansion. The regions in the $\left(\rho_{i}, T_{9 i}\right)$-plane delineated by this restriction are shown in figure 18. This criterion is liberal enough that all runs which burn any significant amount of silicon should attain total silicon-based quasi-equilibrium early in the evolution.

\section{c) Radioactive Progenitors}

In the range of $\eta$ considered to be most appropriate to silicon burning one finds that many species are not produced as their stable isobars but as proton-rich progenitors which presumably decay to the stable species after expulsion from the star. Such was the case in oxygen burning where it was found that ${ }^{37} \mathrm{Cl}$ was made as ${ }^{37} \mathrm{Ar}$ and ${ }^{41} \mathrm{~K}$ was made as ${ }^{41} \mathrm{Ca}$. Since quasi-equilibrium is most likely attained in silicon burning it should be possible to predict the progenitors of the species in the $28 \leq A \leq 66$ range by calculating quasi-equilibrium distributions in the range of expected freeze-out temperatures. Such calculations were performed in the range $T_{9}=2.0$ to $T_{9}=5.0$ for $\eta=0.002$. The primary progenitors of the various stable nuclei are given in table 11 . Stable species not listed are identical with their progenitors. Such information is useful not only in the determination of the proper nuclear reaction network for the generalized computer code but also to predict the probable $\gamma$-ray spectrum of supernova remnants along the lines of Clayton and Craddock (1965), Clayton, Colgate, and Fishman (1969), Clayton and Silk (1969), and Clayton (1971).

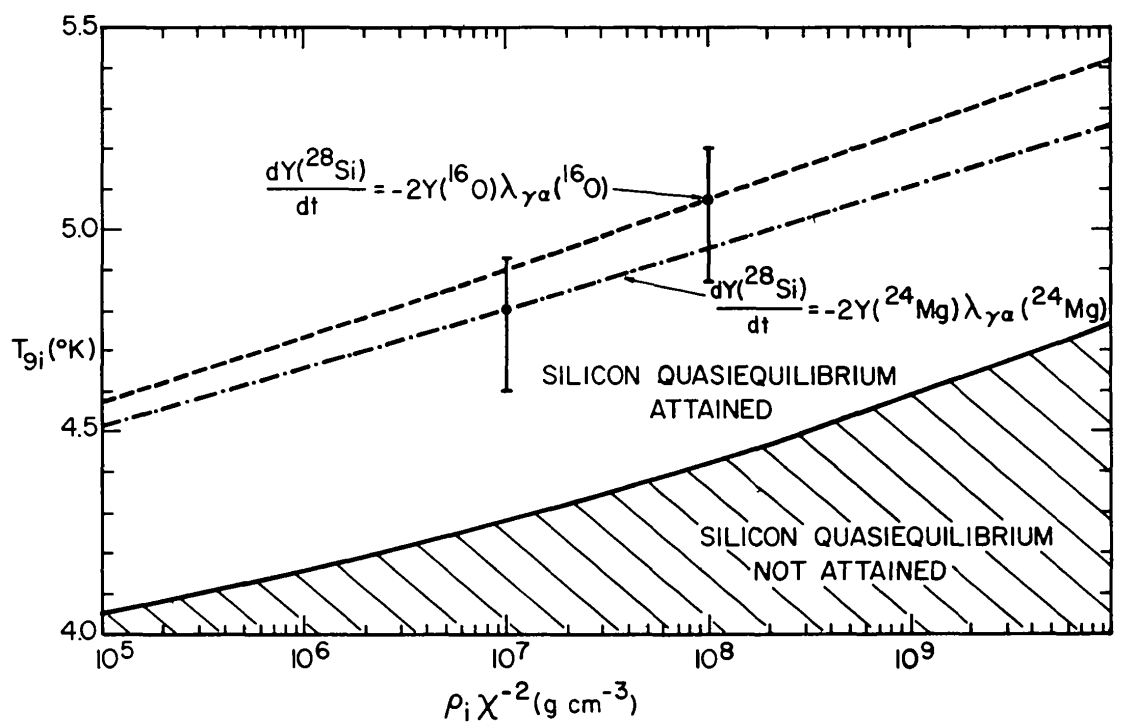

FIG. 18.- Initial conditions for silicon burning. Those initial thermodynamic conditions which will result in the burning of one-half of the initial ${ }^{28} \mathrm{Si}$ abundance are shown for two cases. In one case (dashed line) the rate of silicon burning is being determined by the photodisintegration rate of ${ }^{24} \mathrm{Mg}$; in the other case (broken line) the photodisintegration rate of ${ }^{16} \mathrm{O}$ is governing. Error bars indicate an allowed range of the ratio $X_{f}\left({ }^{28} \mathrm{Si}\right) / X_{i}\left({ }^{28} \mathrm{Si}\right)$ of from 0.1 to 0.9 . The solid line near the bottom segregates those regions of the $\left(\rho_{i} \chi^{-2}, T_{9 i}\right)$-plane where silicon quasi-equilibrium will be attained during the expansion from those where it will not. 
TABLE 11

Most Abundant Radioactive Progenitors of the Terrestrially Stable Nuclei during QUASI-EQUILIBRIUM FOR TEMPERATURES $2.0 \leq T_{9} \leq 8.0$ AND $\eta=0.002$

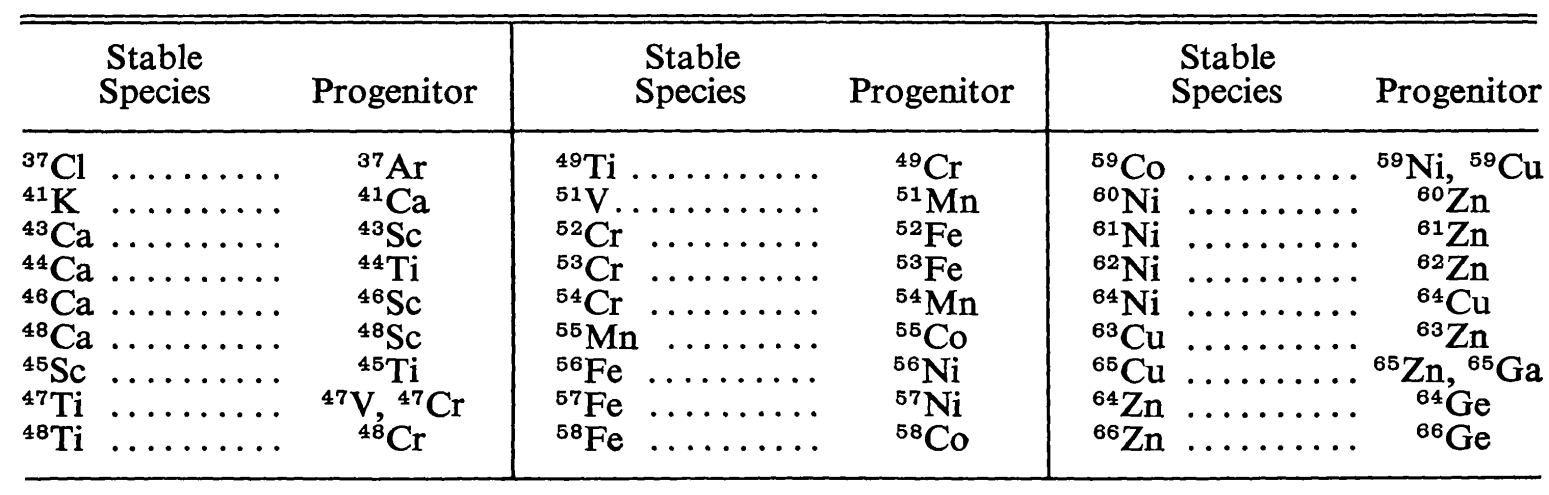

\section{d) Details of Silicon Burning}

If the peak temperature and density in silicon burning are sufficiently moderate so that freeze-out occurs before the silicon abundance is depleted, an abundance distribution will be produced which contains significant amounts of both iron-peak material and intermediate-weight nuclei. The results from a typical evolution resulting in roughly three times as many ${ }^{28} \mathrm{Si}$ nuclei as ${ }^{56} \mathrm{Ni}$ nuclei, but normalized to ${ }^{54} \mathrm{Fe}$, are shown in figure 19. The species ${ }^{28} \mathrm{Si},{ }^{32} \mathrm{~S},{ }^{36} \mathrm{Ar},{ }^{40} \mathrm{Ca},{ }^{48} \mathrm{Ti},{ }^{49} \mathrm{Ti},{ }^{50} \mathrm{Cr},{ }^{52} \mathrm{Cr},{ }^{53} \mathrm{Cr}$, and ${ }^{55} \mathrm{Mn}$ are all produced relative to ${ }^{54} \mathrm{Fe}$ in amounts in accord with the solar-system values to within a factor of 2 . Somewhat smaller amounts of ${ }^{44} \mathrm{Ca},{ }^{51} \mathrm{~V},{ }^{56} \mathrm{Fe},{ }^{57} \mathrm{Fe}$, and ${ }^{58} \mathrm{Ni}$ are also produced. The yields of ${ }^{56} \mathrm{Fe}$ and ${ }^{57} \mathrm{Fe}$ increase as more silicon is burned, whereas the ${ }^{54} \mathrm{Fe}$ yield remains relatively constant because it depends primarily on the value of $\eta$. The coproduction of ${ }^{28} \mathrm{Si},{ }^{32} \mathrm{~S},{ }^{36} \mathrm{Ar}$, and ${ }^{40} \mathrm{Ca}$ is of course no great surprise as it reflects the same equilibrium with respect to $\alpha$-particles that is established during explosive oxygen burning.

Although the peak temperature for figure 19 was $T_{9}=4.7$ the overall distribution is very similar to the quasi-equilibrium abundances calculated at a freezing temperature $T_{9 f}=3.5$. Other (nonequilibrium) freeze-out effects seem to be small, particularly for abundant species. The situation is analogous to that of explosive oxygen burning; viz., evolutions that freeze out with the appropriate alpha density and $\eta$ should give approximately the same distribution. This similarity motivates a survey of the alpha densities necessary to reproduce various solar-system abundance ratios. The reasoning and procedure follow that of $\S \mathrm{IV} d$. In table 12 the values of $\log \rho X_{\alpha}$ required to reproduce various alpha-related ratios correctly are given, assuming the progenitors listed in table 11. Also given are the values of $\log \rho X_{\alpha}$ necessary to produce the solar number ratios of ${ }^{56} \mathrm{Fe},{ }^{52} \mathrm{Cr}$, and ${ }^{48} \mathrm{Ti}$ relative to ${ }^{28} \mathrm{Si}$ at freeze-out. From the table, it seems apparent that there exist at least three groups having members which can consistently be coproduced for three different appropriate freeze-out alpha densities. One of these groups, containing most of the species produced in silicon burning, may be correctly reproduced by an alpha density which implies approximately equal amounts of ${ }^{56} \mathrm{Fe}$ and ${ }^{28} \mathrm{Si}$.

For realistic freeze-out temperatures the alpha density implied by the numberdensity ratio ${ }^{56} \mathrm{Fe} /{ }^{28} \mathrm{Si}=0.88$ is actually somewhat large for the consistent reproduction of ${ }^{48} \mathrm{Ti} /{ }^{44} \mathrm{Ca},{ }^{52} \mathrm{Cr} /{ }^{48} \mathrm{Ti}$, and ${ }^{54} \mathrm{Fe} /{ }^{50} \mathrm{Cr}$. A value about $0.6 \operatorname{lower}$ in $\log \rho X_{\alpha}$ at $T_{9 f}=3.4$, for example, would give a more nearly correct reproduction of these ratios. However, because the abundance of ${ }^{56} \mathrm{Fe}$ relative to that of ${ }^{28} \mathrm{Si}$ is proportional to the seventh power of the alpha density, decreasing $\log \rho X_{\alpha}$ by anything like 0.6 would mean that almost no mass is contained in the iron peak. It seems that one is stuck with 


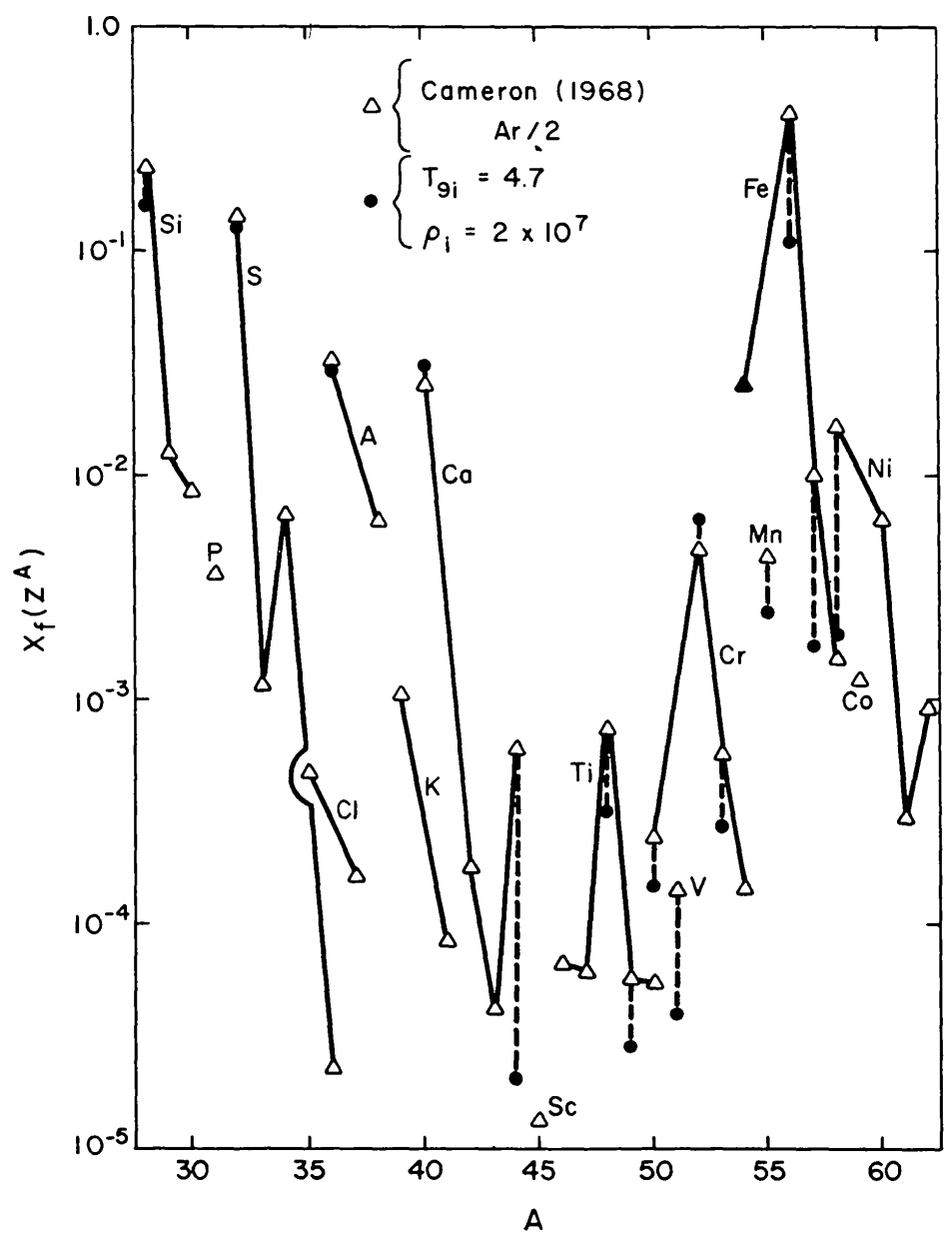

FIG. 19.- Final abundances resulting from a typical case of silicon burning. The final abundances by mass fraction of the species $Z^{A}$ resulting from the explosive burning of composition " $O$ " are shown compared to the solar abundance distribution. The initial conditions of the run are $T_{9 i}=$ $4.7, \rho_{i}=2.0 \times 10^{7}, \chi=4.45$, and $\eta=0.002$. The progenitors of the stable species are those elements listed in table 11. Abundances having mass fractions less than $10^{-5}$ are not indicated.

the slightly high value. This implies, for example, that any run that makes ${ }^{54} \mathrm{Fe}$ and ${ }^{52} \mathrm{Cr}$ in the correct amounts (as in fig. 19) should underproduce ${ }^{50} \mathrm{Cr},{ }^{56} \mathrm{Fe},{ }^{48} \mathrm{Ti}$, and ${ }^{44} \mathrm{Ca}$. A run that makes ${ }^{55} \mathrm{Mn}$ correctly will slightly underproduce ${ }^{51} \mathrm{~V}$ and greatly underproduce ${ }^{47} \mathrm{Ti}$. Any attempt to correct these underproductions by lowering $\log \rho X_{\alpha}$ will result in too little material being processed into the iron peak to account for abundant species relative to ${ }^{28} \mathrm{Si}$. It is also apparent that species of $A>56$ will be underproduced requiring as they do a $\rho X_{\alpha}$ some two to eight orders of magnitude larger than that obtained in silicon burning.

It seems that for reasonable freeze-out temperatures, say $T_{9} \sim 3.4$ and hence for reasonable expansion time scales, one is stuck with the underproductions seen in figure 19. In some cases, for example ${ }^{51} \mathrm{~V},{ }^{50} \mathrm{Cr}$, and ${ }^{48} \mathrm{Ti}$, the underproductions are not too severe. In other cases, either the abundances of the species must be favorably modified by some sort of freeze-out conditions other than those resulting in figure 19, or the species must be produced elsewhere. The most important underproductions of silicon burning are the major nuclei ${ }^{44} \mathrm{Ca},{ }^{54} \mathrm{Cr},{ }^{58} \mathrm{Fe}$, and ${ }^{58} \mathrm{Ni}$. The last will come naturally from nuclear statistical equilibria, as Clayton and Woosley (1969) and ATW have argued, but the other three remain somewhat mysterious. 
TABLE 12

VALUES OF $\log _{10} \rho \mathrm{X}_{\alpha}$ IMPLIED BY VARIOUS ABUNDANCE RATIOS

(Progenitors are given in table 11 )

\begin{tabular}{|c|c|c|c|c|c|c|c|c|c|c|}
\hline $\begin{array}{l}\text { mber } \\
\text { tio }\end{array}$ & ${ }^{56} \mathrm{Fe} /{ }^{28} \mathrm{Si}$ & ${ }^{48_{\mathrm{T} i} /{ }^{28} \mathrm{Si}}$ & ${ }^{52} \mathrm{cr} /{ }^{28} \mathrm{Si}$ & ${ }^{54} \mathrm{Fe} /{ }^{50} \mathrm{Cr}$ & ${ }^{55} \mathrm{Mn} /{ }^{51} \mathrm{v}$ & ${ }^{52} \mathrm{Cr} / /^{48} \mathrm{Ti}$ & ${ }^{56} \mathrm{Fe} /{ }^{52} \mathrm{Cr}$ & $48_{\mathrm{Ti} /}^{44} \mathrm{Ca}$ & $51_{\mathrm{V} /} /{ }^{\mathrm{Ti}}$ & ${ }^{53} \mathrm{Cr} /{ }^{49} \mathrm{Ti}$ \\
\hline $\begin{array}{l}\text { lar } \\
\text { lue }\end{array}$ & 0.88 & 0.0019 & 0.011 & 9.7 & 29.4 & 6.1 & 78.4 & 1.1 & 1.8 & 9.29 \\
\hline \multicolumn{11}{|l|}{ ' $9 f$} \\
\hline .6 & -1.62 & -1.49 & -1.57 & -2.87 & -2.52 & -2.54 & -1.54 & -2.59 & -4.60 & -2.08 \\
\hline .8 & -.60 & -.51 & -.67 & -1.66 & -1.34 & -1.39 & -.38 & -1.49 & -3.15 & -.96 \\
\hline .0 & .29 & .34 & .22 & -.60 & -.31 & -.40 & .62 & -.53 & -2.27 & 0 \\
\hline .2 & 1.07 & 1.08 & .99 & .32 & .59 & .48 & 1.50 & .31 & -1.31 & .86 \\
\hline .4 & 1.77 & 1.74 & 1.67 & 1.14 & 1.38 & 1.26 & 2.28 & 1.05 & -.47 & 1.62 \\
\hline .6 & 2.38 & 2.33 & 2.28 & 1.87 & 2.10 & 1.95 & 2.98 & 1.71 & .28 & 2.29 \\
\hline .8 & 2.94 & 2.86 & 2.82 & 2.53 & 2.73 & 2.57 & 3.61 & 2.30 & .96 & 2.90 \\
\hline .0 & 3.45 & 3.37 & 3.31 & 3.12 & 3.31 & 3.13 & 4.17 & 2.84 & 1.57 & 3.45 \\
\hline .2 & 3.90 & 3.77 & 3.76 & 3.50 & 3.83 & 3.64 & 4.68 & 3.33 & 2.12 & 3.94 \\
\hline 1.4 & 4.32 & 4.16 & 4.16 & 4.15 & 4.31 & 4.10 & 5.15 & 3.77 & 2.62 & 4.39 \\
\hline .6 & 4.70 & 4.52 & 4.53 & 4.60 & 4.75 & 4.52 & 5.57 & 4.18 & 3.18 & 4.81 \\
\hline$\vdots .8$ & 5.05 & 4.86 & 4.88 & 5.01 & 5.15 & 4.92 & 5.97 & 4.55 & 3.51 & 5.19 \\
\hline 1.0 & 5.37 & 5.16 & 5.19 & 5.39 & 5.52 & 5.28 & 6.33 & 4.90 & 3.90 & 5.54 \\
\hline $\begin{array}{l}\text { imber } \\
\text { itio }\end{array}$ & ${ }^{58} \mathrm{Ni} /{ }^{54} \mathrm{Fe}$ & ${ }^{60} \mathrm{Ni} /{ }^{56} \mathrm{Fe}$ & ${ }^{62} \mathrm{Ni} /{ }^{58} \mathrm{Ni}$ & ${ }^{6 I_{\mathrm{Ni}} /{ }^{57} \mathrm{Fe}}$ & ${ }^{63} \mathrm{Cu} /{ }^{59} \mathrm{Co}$ & ${ }^{59} \mathrm{Co} /{ }^{55} \mathrm{Mn}$ & ${ }^{64} \mathrm{zn} /{ }^{60} \mathrm{Ni}$ & ${ }^{66} \mathrm{zn} /{ }^{62} \mathrm{Ni}$ & ${ }^{67} \mathrm{zn} /{ }^{63} \mathrm{Cu}$ & \\
\hline $\begin{array}{l}\text { Ilar } \\
\text { llue }\end{array}$ & 0.60 & 0.015 & 0.054 & 0.028 & 0.28 & 0.26 & 0.061 & 0.25 & 0.097 & \\
\hline \multicolumn{11}{|l|}{$\Gamma_{9 f}$} \\
\hline 2.6 & -.57 & 4.98 & 4.37 & 4.90 & 4.77 & 2.54 & 6.47 & 7.52 & 5.86 & \\
\hline$? .8$ & .36 & 5.41 & 4.88 & 5.35 & 5.30 & 3.25 & 6.83 & 7.85 & 6.28 & \\
\hline 3.0 & 1.18 & 5.78 & 5.32 & 5.74 & 5.76 & 3.87 & 7.15 & 8.14 & 6.64 & \\
\hline 3.2 & 1.89 & 6.11 & 5.71 & 6.09 & 6.17 & 4.41 & 7.43 & 8.40 & 6.96 & \\
\hline 3.4 & 2.53 & 6.40 & 6.06 & 6.40 & 6.53 & 4.89 & 7.68 & 8.62 & 7.25 & \\
\hline 3.6 & 3.09 & 6.66 & 6.37 & 6.67 & 6.85 & 5.32 & 7.90 & 8.83 & 7.51 & \\
\hline 3.8 & 3.60 & 6.89 & 6.65 & 6.92 & 7.15 & 5.71 & 8.11 & 9.02 & 7.74 & \\
\hline 7.0 & 4.06 & 7.11 & 6.91 & 7.15 & 7.41 & 6.06 & 8.29 & 9.18 & 7.95 & \\
\hline 4.2 & 4.47 & 7.30 & 7.14 & 7.35 & 7.65 & 6.38 & 8.46 & 9.34 & 8.14 & \\
\hline 1.4 & 4.85 & 7.48 & 7.35 & 7.54 & 7.87 & 6.67 & 8.61 & 9.48 & 8.32 & \\
\hline 7.6 & 5.20 & 7.64 & 7.54 & 7.71 & 8.07 & 6.94 & 8.75 & 9.61 & 8.48 & \\
\hline 4.8 & 5.52 & 7.79 & 7.72 & 7.87 & 8.26 & 7.18 & 8.88 & 9.73 & 8.63 & \\
\hline 5.0 & 5.82 & 7.93 & 7.89 & 8.02 & 8.43 & 7.41 & 9.01 & 9.84 & 8.77 & \\
\hline
\end{tabular}

The species that are produced in silicon burning can generally be produced by any run that is characterized by roughly equal amounts of ${ }^{28} \mathrm{Si}$ and ${ }^{56} \mathrm{Ni}$ at freeze-out. The conditions yielding figure 19 are certainly not unique. In order to determine what range of peak conditions are appropriate to silicon burning under our explosive assumptions, it is necessary to have an equation which specifies, at least approximately, the time evolution of the ${ }^{28} \mathrm{Si}$ abundance.

In what follows we derive approximations adequate to ascertain those explosive conditions that will burn roughly half the silicon. This result allows us to estimate what stars must do in order to produce results similar to figure 19. We will use the result first described by BCF; viz., the rate of silicon burning lies between the value obtained when ${ }^{24} \mathrm{Mg}$ is in quasi-equilibrium with ${ }^{28} \mathrm{Si}$ and the value when ${ }^{16} \mathrm{O}$ is.

If ${ }^{24} \mathrm{Mg}(\gamma, \alpha){ }^{20} \mathrm{Ne}$ is the nonequilibrium link determining the rate of silicon burning, 
then $\mathrm{BCF}$ find that an approximate equation for the ${ }^{28} \mathrm{Si}$ lifetime is given by

$$
\frac{1}{\tau\left({ }^{28} \mathrm{Si}\right)} \simeq 10^{15.88}\left(\frac{T_{9}}{4}\right)^{3 / 2}\left[\frac{2 X\left({ }^{28} \mathrm{Si}\right)}{1-X\left({ }^{28} \mathrm{Si}\right)}\right]^{1 / 7} e^{-157.5 / T_{9} \mathrm{~S}^{-1}},
$$

where $X\left({ }^{28} \mathrm{Si}\right)$ is the ${ }^{28} \mathrm{Si}$ mass fraction. For purposes of the present calculation it will suffice to take

$$
\left[\frac{2 X\left({ }^{28} \mathrm{Si}\right)}{1-X\left({ }^{28} \mathrm{Si}\right)}\right]^{1 / 7} \simeq 1
$$

then fitting a power law to the above equation near $T_{9}=4.5$ one has approximately that

$$
\frac{d Y\left({ }^{28} \mathrm{Si}\right)}{d t} \simeq-7.8 \times 10^{-25} T_{9}^{38} Y\left({ }^{28} \mathrm{Si}\right) \mathrm{s}^{-1}
$$

which integrates to

$$
\ln \frac{Y_{f}\left({ }^{28} \mathrm{Si}\right)}{\left.Y_{i}{ }^{28} \mathrm{Si}\right)}=-2.7 \times 10^{-23} \chi \rho_{i}{ }^{-1 / 2} T_{9 i}{ }^{38},
$$

where $Y_{i}\left({ }^{28} \mathrm{Si}\right)$ and $Y_{f}\left({ }^{28} \mathrm{Si}\right)$ are the initial and final $Y$ 's of ${ }^{28} \mathrm{Si}$. This implies that the demands of silicon burning will be satisfied for

$$
\rho_{i} \chi^{-2} \simeq 7.5 \times 10^{-46} T_{9 i}{ }^{76}\left[\ln \frac{Y_{f}\left({ }^{28} \mathrm{Si}\right)}{Y_{i}\left({ }^{28} \mathrm{Si}\right)}\right]^{-2}
$$

or

$$
\rho_{i} \chi^{-2} \simeq 1.6 \times 10^{-45} T_{9 i}{ }^{76}
$$

if about $\frac{1}{2}$ of the original ${ }^{28} \mathrm{Si}$ is consumed. If instead one assumes that ${ }^{16} \mathrm{O}(\gamma, \alpha){ }^{12} \mathrm{C}$ is determining the rate of silicon burning a similar derivation shows the lifetime of ${ }^{28} \mathrm{Si}$ is determined by

$$
\begin{aligned}
\frac{d Y\left({ }^{28} \mathrm{Si}\right)}{d t} & =\frac{Y\left({ }^{28} \mathrm{Si}\right)}{\tau\left({ }^{28} \mathrm{Si}\right)} \simeq-2 Y\left({ }^{16} \mathrm{O}\right) \lambda_{\gamma \alpha}\left({ }^{16} \mathrm{O}\right) \\
& =4.24 \times 10^{32} \frac{T_{9}^{9 / 2}}{\rho^{3} Y_{\alpha}{ }^{3}} e^{-278.86 / T_{9}} Y\left({ }^{28} \mathrm{Si}\right) \lambda_{\gamma \alpha}\left({ }^{16} \mathrm{O}\right),
\end{aligned}
$$

where $Y\left({ }^{16} \mathrm{O}\right)$ has been set in quasi-equilibrium with $Y\left({ }^{28} \mathrm{Si}\right)$. Inserting our rate for $\lambda_{\gamma \alpha}\left({ }^{16} \mathrm{O}\right)$ the power-law approximation becomes

$$
\frac{1}{\tau\left({ }^{28} \mathrm{Si}\right)} \simeq 2.0 \times 10^{-22} T_{9}{ }^{34}\left[\frac{2 X\left({ }^{28} \mathrm{Si}\right)}{1-X\left({ }^{28} \mathrm{Si}\right)}\right]^{3 / 7} .
$$

Again assuming that $X\left({ }^{28} \mathrm{Si}\right)$ is not too far from $\frac{1}{3}$ so that the factor $\left\{\left[2 X\left({ }^{28} \mathrm{Si}\right)\right] /\right.$ $\left.\left[1-X\left({ }^{28} \mathrm{Si}\right)\right]\right\}^{3 / 7}$ is about equal to 1 , one obtains

$$
\ln \frac{Y_{f}\left({ }^{28} \mathrm{Si}\right)}{Y_{i}\left({ }^{28} \mathrm{Si}\right)} \simeq-7.9 \times 10^{-21} \chi \rho_{i}{ }^{-1 / 2} T_{9 i}{ }^{34} .
$$


Hence equation (54) becomes instead

$$
\rho_{i} \chi^{-2} \simeq 6.2 \times 10^{-41} T_{9 i}{ }^{68}\left[\ln \frac{Y_{f}\left({ }^{28} \mathrm{Si}\right)}{Y_{i}\left({ }^{28} \mathrm{Si}\right)}\right]^{-2}
$$

or

$$
\rho_{i} \chi^{-2} \simeq 1.2 \times 10^{-40} T_{9 i}{ }^{68}
$$

if about $\frac{1}{2}$ of the original ${ }^{28} \mathrm{Si}$ is to be consumed.

Equations (54) and (58) provide alternate expressions for conditions that burn half the silicon, and the correct value is expected to lie between them. These two conditions are plotted in figure 18. The band of temperatures and densities lying between them is entirely in the region where quasi-equilibrium is attained. Accordingly we conclude that, except for freeze-out effects, quasi-equilibrium is a reasonably good model for silicon burning in those cases where approximately half the silicon is consumed.

\section{SILICON EXHAUSTION AND NUCLEAR STATISTICAL EQUILIBRIUM}

Depletion of ${ }^{28} \mathrm{Si}$ will occur during a given nuclear evolution if the lifetime of ${ }^{28} \mathrm{Si}$ to silicon burning is much less than the expansion time scale under the given conditions, that is if $\tau\left({ }^{28} \mathrm{Si}\right) \ll \tau_{\mathrm{HD}}$. A quantitative estimate of the conditions implied by this inequality may be obtained by examination of the differential equation for the abundance of ${ }^{28} \mathrm{Si}$. Due to the high temperatures required for complete explosive consumption of silicon it is probably more realistic to take the rate of silicon burning to be determined by ${ }^{16} \mathrm{O}$ rather than ${ }^{24} \mathrm{Mg}$ photodisintegration. For small $X\left({ }^{28} \mathrm{Si}\right)$ then equations (2), (3), and (56) give

$$
\frac{d X\left({ }^{28} \mathrm{Si}\right)}{d T_{9}} \simeq 8.0 \times 10^{-22}\left[X\left({ }^{28} \mathrm{Si}\right)\right]^{10 / 7} T_{9}{ }^{33} \tau_{\mathrm{HD}} .
$$

Integration of this equation provides an approximate representation of the evolution of the silicon abundance for low values of $X\left({ }^{28} \mathrm{Si}\right)$. Neglecting inverse powers of the initial silicon abundance as compared to the inverse of the much smaller final silicon mass fraction one has the final silicon abundance

$$
X_{f}\left({ }^{28} \mathrm{Si}\right) \simeq\left(1.0 \times 10^{-23} T_{9 i}{ }^{34} \tau_{\mathrm{HD}}\right)^{-7 / 3} .
$$

Once the silicon mass fraction drops below about 0.005 it no longer controls the character of the evolution. Defining this as the point of silicon exhaustion, equation (60) specifies that complete silicon burning will occur for initial conditions satisfying

$$
T_{9 i} \geqslant 4.3\left(\frac{\rho_{i}}{\chi^{2}}\right)^{1 / 68},
$$

the actual value being relatively insensitive to what one takes for $X_{f}\left({ }^{28} \mathrm{Si}\right)$. The region specified by this equation is shown in figure 20 for two different time-scale parameters. To obtain silicon exhaustion for reasonable densities, the initial temperature must exceed $5.0 \times 10^{\circ} \mathrm{K}$.

Since in the quasi-equilibrium, which one would expect to be quickly established under such conditions,

$$
\rho N_{\mathrm{A}} Y_{\alpha}=\left[C_{56}{ }^{-1}\left(T_{9}\right) \frac{Y\left({ }^{56} \mathrm{Ni}\right)}{Y\left({ }^{28} \mathrm{Si}\right)}\right]^{1 / 7},
$$

depletion of ${ }^{28} \mathrm{Si}$ implies increasing alpha density. This alpha density becomes large 


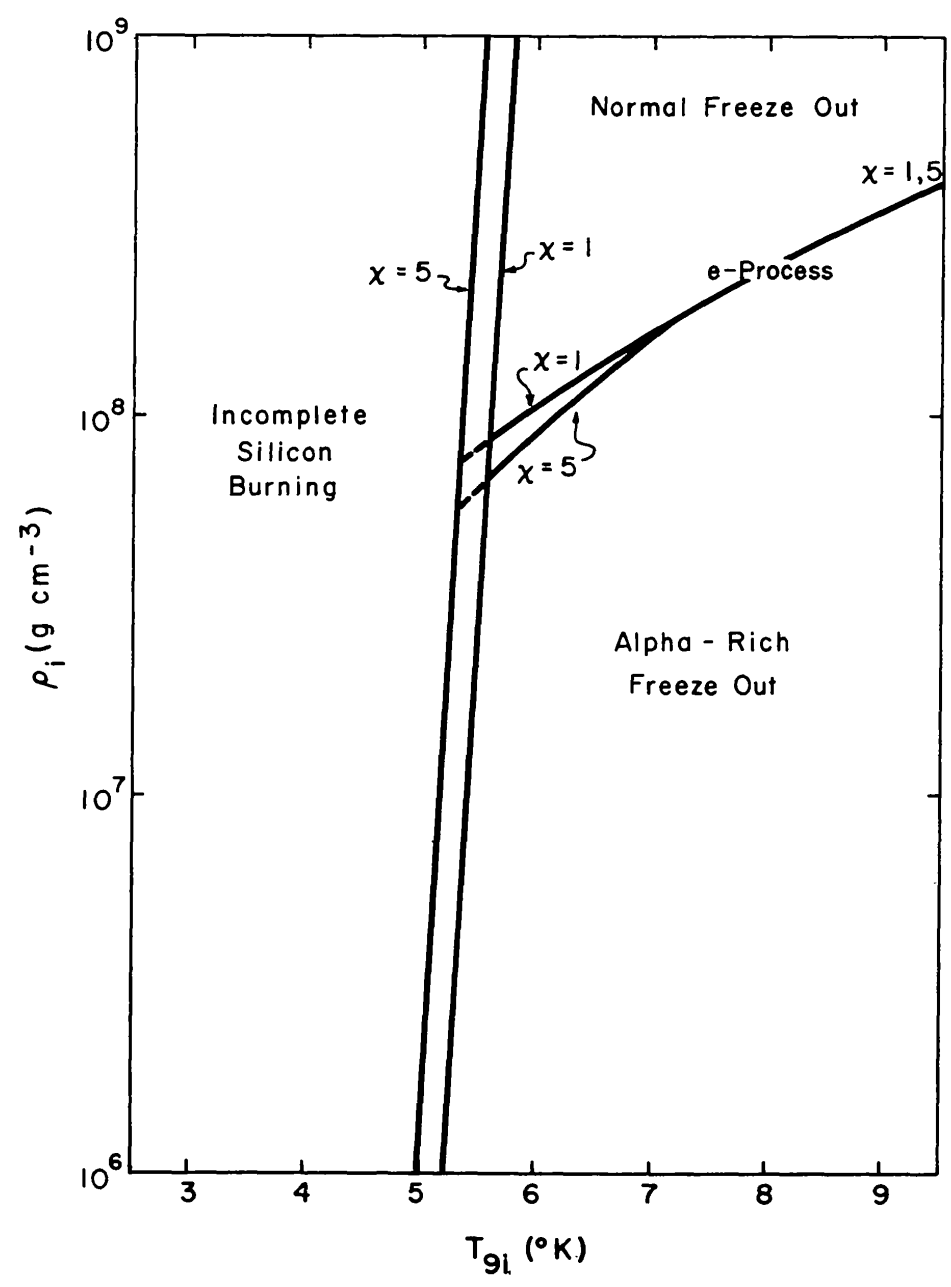

FIG. 20.-Peak thermodynamic conditions for various types of silicon burning. The lines in the vicinity of $T_{9 i}=5.0$ segregate the $\left(\rho_{i}, T_{9 i}\right)$-plane into those regions where silicon exhaustion or silicon burning will occur. The delineation varies somewhat with the time scale. Two lines characterized by different time-scale parameters, $\chi$, are shown. The region where silicon exhaustion occurs is further subdivided according to the expected nature of the freeze-out. For densities to the high-density side of the lines in the vicinity of $\rho_{i}=10^{8}$ the freeze-out should be "normal" and the resultant abundances similar to those obtained in silicon burning. Lower densities will result in a freeze-out characterized by high free-particle abundances. Lines for two values of the time-scale parameter are shown.

enough so that the triple-alpha reaction comes into equilibrium with ${ }^{12} \mathrm{C}$ photodisintegration; that is $r_{3 \alpha}=Y\left({ }^{12} \mathrm{C}\right) \lambda_{\gamma 3 \alpha}\left({ }^{12} \mathrm{C}\right)$. In such cases all other reactions have usually already come into equilibrium. Thus the conditions for nuclear statistical equilibrium (the $e$-process) are satisfied. From this point on, until some reaction falls out of equilibrium, the entire composition may be specified by only three parameters which one may take to be $T_{9}, \rho$, and $\eta$. Since $\rho$ and $T_{9}$ are assumed given in terms of the time and initial conditions by equations (2) and (3), the composition at any point in time is specified by only $\eta$ and the initial conditions. The alpha mass fraction in nuclear statistical equilibrium is shown as a function of $T_{9}$ and $\rho$ for $\eta=0.002$ in figure 21. For conditions sufficient to result in silicon exhaustion (fig. 20) it appears that an alpha mass fraction of at least several percent will exist during equilibrium. In the lower range of densities and/or for temperatures in excess of the minimum required to achieve equilibrium, the alpha mass fraction will be larger.

As the temperature falls reaction links begin to come out of equilibrium. The first 


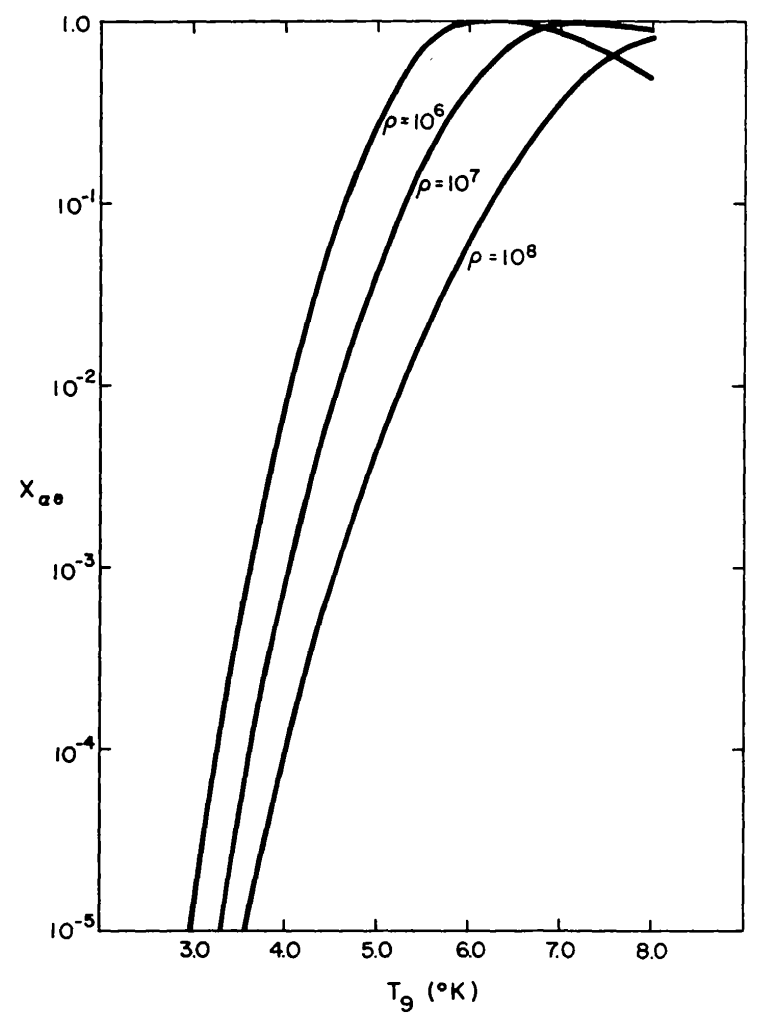

FIG. 21.-Alpha mass fraction in total nuclear statistical equilibrium. The abundance of $\alpha-$ particles by mass is shown as a function of the temperature, $T_{\theta}$, for various densities for the special case $\eta=0.002$.

links to drop out are those involving $\alpha$-particles. This is chiefly due to the high Coulomb barrier involved in such reactions.

\section{a) Normal Freeze-out}

Alpha-particle freeze-out manifests itself in either of two ways. Let the temperature at which the critical alpha reactions begin to drop out of equilibrium be defined as $T_{9 \alpha}$. If the density at $T_{9 \alpha}$ is large enough so that the alpha mass fraction is small, say $X_{\alpha}<0.01$, then the termination of equilibrium may be due to a deficiency in $\alpha$ particles. In the appropriate temperature region the photodisintegration rates are more temperature sensitive than the forward rates involving $\alpha$-particles. At $T_{9 \alpha}$ the photodisintegration rates cease to be efficient enough to provide the $\alpha$-particles necessary for rearrangement into a new equilibrium distribution in the time available. All the $\alpha$-particles are bound into species that are characteristic of statistical equilibrium at a higher temperature. Generally, the effect of this "low alpha density" is seen first in the triple-alpha reaction which falls out of equilibrium with ${ }^{12} \mathrm{C}$ photodisintegration resulting in an anomalously low abundance for ${ }^{12} \mathrm{C}$ compared to the equilibrium value. Later critical links among the various alpha nuclei, ${ }^{16} \mathrm{O},{ }^{20} \mathrm{Ne}$, ${ }^{24} \mathrm{Mg}$, etc., fall out. The net result is that material in the lighter alpha nuclei is not consolidated into the iron peak at low temperatures as much as nuclear statistical equilibrium would predict. Thus abundances of lighter alpha nuclei at temperatures lower than $T_{9 \alpha}$ will be much higher than one might expect from purely statistical considerations.

Once the evolution drops out of equilibrium, its future history will depend in a critical fashion upon the free-particle densities at that point and the time scale for 


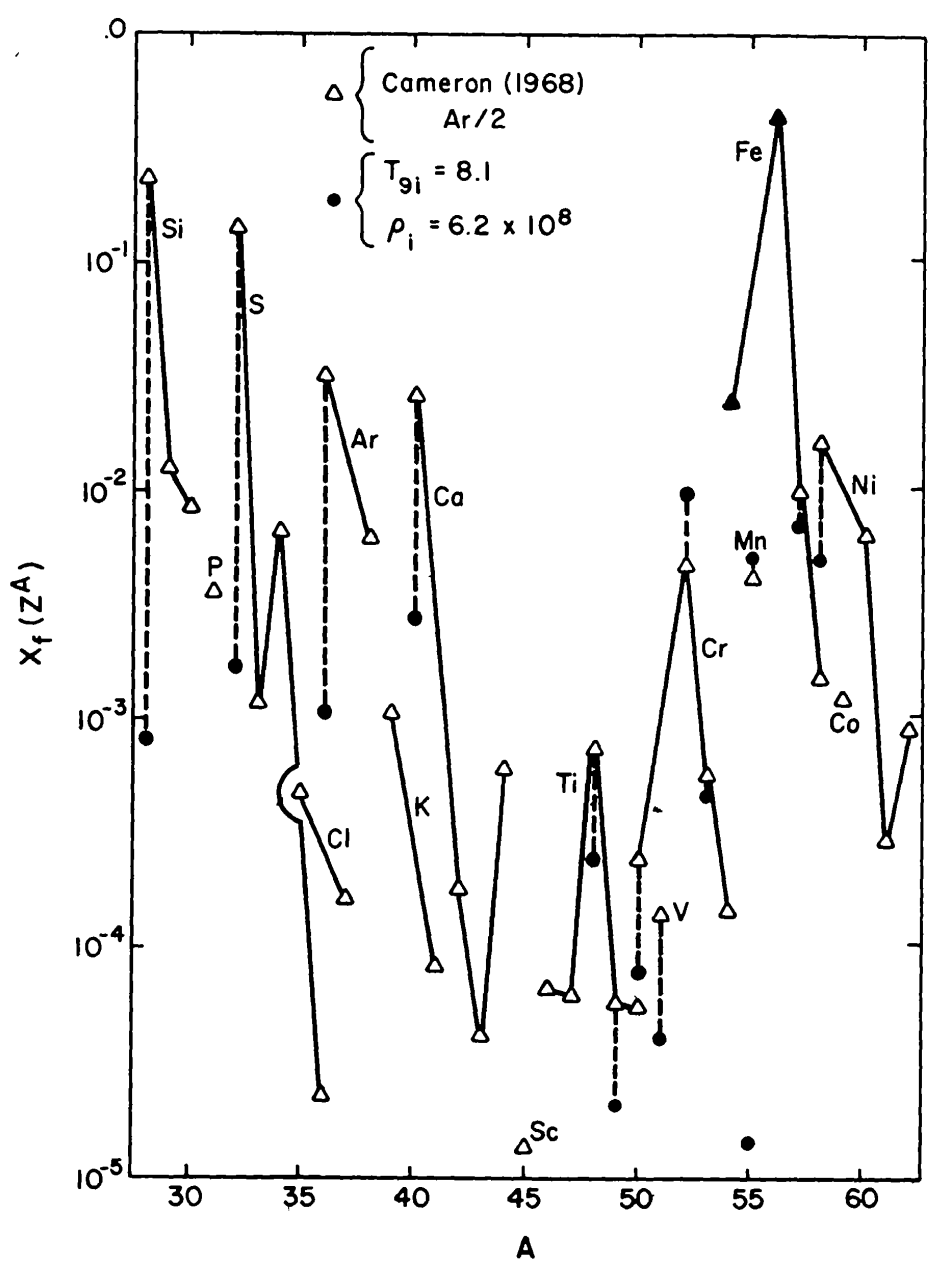

Fig. 22.-Final abundances resulting from the e-process with a normal freeze-out. The final abundances of the species $Z^{A}$ resulting from the explosive burning of composition "Si" are shown compared to the solar abundance distribution. The results have been normalized to ${ }^{56} \mathrm{Fe}$. The initial conditions of the run were $T_{9 i}=8.1, \rho_{i}=6.2 \times 10^{8}, \chi=1.0$, and $\eta=0.002$. The progenitors of these stable species are the elements listed in table 11. Abundances having mass fractions less than $10^{-5}$ are not indicated. The final mass fraction of free $\alpha$-particles was $X_{\alpha}=7.2 \times$ $10^{-13}$

freeze-out from that point. If the density is high enough so that the free-particle densities at $T_{9} \simeq 5.0$ are small or if the time scale for freeze-out from this temperature is long enough so that all free particles can be reincorporated into bound nuclei, then a "normal freeze-out" will occur. The resultant abundance distribution will closely resemble that obtained from silicon burning having small final ${ }^{28} \mathrm{Si}$ mass fractions. The results of such a run are shown in figure 22. There is nothing there worthy of mention that reflects the different history of this run from that of a silicon-burning run ending with $X_{f}\left({ }^{28} \mathrm{Si}\right) \simeq 10^{-3}$.

\section{b) Alpha-rich Freeze-out}

Just the opposite effect occurs at low densities. Here the alpha density is so high that the end of equilibrium is caused by the "freezing out" of the forward reactions which involve $\alpha$-particles. This results in an inability on the part of the abundance distribution to react as quickly to a change in temperature and density as statistical equilibrium would have demanded that it does. Expressed more quantitatively, to 
remain in statistical equilibrium the alpha density must be able to decrease at a rate

$$
\left(\frac{d Y_{\alpha}}{d t}\right)_{e}=\left(\frac{\partial Y_{\alpha}}{\partial \rho}\right)_{e} \frac{\partial \rho}{\partial t}+\left(\frac{\partial Y_{\alpha}}{\partial T_{9}}\right)_{e} \frac{\partial T_{9}}{\partial t}+\left(\frac{\partial Y_{\alpha}}{\partial \eta}\right)_{e} \frac{\partial \eta}{\partial t},
$$

where the subscript $e$ indicates that the partial derivatives are to be evaluated by a nuclear statistical equilibrium calculation. Both the dependence of $Y_{\alpha}$ on $\eta$ and the time derivative of $\eta$ are small. Using equations (2) and (3) the above derivative may be expressed as

$$
\left(\frac{d Y_{\alpha}}{d t}\right)_{e} \simeq-\frac{1}{\tau_{\mathrm{HD}}}\left[\rho\left(\frac{\partial Y_{\alpha}}{\partial \rho}\right)_{e}+\frac{T_{9}}{3}\left(\frac{\partial Y_{\alpha}}{\partial T_{9}}\right)_{e}\right]
$$

As the temperature decreases, the tendency of the $\alpha$-particles is to merge back into the iron peak. This tendency is confirmed by an examination of several calculations at $T_{9 \alpha}$. Most of the $\alpha$-particles are going back into ${ }^{56} \mathrm{Ni}$ via the alpha-consuming reaction chain $\alpha(2 \alpha, \gamma)^{12} \mathrm{C}(\alpha, \gamma)^{16} \mathrm{O} \ldots{ }^{52} \mathrm{Fe}(\alpha, \gamma)^{56} \mathrm{Ni}$. The critical link in this chain is the triple-alpha reaction. Thus the maximum rate at which the $\alpha$-particles may actually be reincorporated (neglecting ${ }^{12} \mathrm{C}$ photodisintegration) is

$$
\left(\frac{d Y_{\alpha}}{d t}\right)_{\max } \simeq 14 r_{3 \alpha}=14\left(\frac{1}{6} Y_{\alpha}^{3} \rho^{2} N_{\mathrm{A}}^{2}\langle\sigma v\rangle_{3 \alpha}\right)
$$

When

$$
\left|\left(\frac{d Y_{\alpha}}{d t}\right)_{e}\right|>\left|\left(\frac{d Y_{\alpha}}{d t}\right)_{\max }\right|
$$

nuclear statistical equilibrium can no longer be valid. The actual $\alpha$-particle density will be too high. The effect is again first felt in the critical ${ }^{12} \mathrm{C}$ link. The photodisintegration of ${ }^{12} \mathrm{C}$ becomes completely overshadowed by the triple-alpha reaction. Because the free-alpha density is "too large," an overabundance of heavy species relative to lighter ones results.

In both the high- and low-density cases, the breakdown of statistical equilibrium usually occurs at $T_{9} \simeq 5.0$. Below this temperature true statistical equilibrium will usually not hold for all species; however, quasi-equilibrium with the free particles may still be a good approximation in certain blocks which are held in quasi-equilibrium by neutron and proton reactions.

If freeze-out occurs before the free particles can all be incorporated, there will be anomalously large free-particle densities present even at low temperatures. This is interesting in light of the quasi-equilibrium demands of table 12 which show very high particle densities are favorable to the production of many nickel, copper, and zinc isotopes. This possibility should be treated with care, however. With such large freeparticle densities the quasi-equilibrium abundances may undergo great change during freeze-out.

In order for this "alpha-rich freeze-out" to occur, two conditions must be satisfied: (1) the $\alpha$-particle density at $T_{9 \alpha}$ must be large, say $X_{\alpha}>0.01$, and (2) this $\alpha$-particle density must not be depletted before the final freeze-out occurs. Taking $T_{9 \alpha} \simeq 5$ the first condition requires densities at $T_{9}=5$ less than about $6 \times 10^{7} \mathrm{~g} \mathrm{~cm}^{-3}$ (see fig. 21). The second condition may be mathematically stated

$$
\int_{T_{9 \alpha}}^{0}\left(\frac{d X_{\alpha}}{d t}\right)\left(\frac{d t}{d T_{9}}\right) d T_{9}<X_{\alpha}\left(T_{9 \alpha}\right)
$$

If the time evolution of the alpha density may be approximated by equation (65), as is 
usually appropriate for high alpha densities during freeze-out, then the final $\alpha$-particle abundance $X_{\alpha f}$ is given by

$$
X_{\alpha f}^{-2}=X_{\alpha}^{-2}\left(T_{9 \alpha}\right)+2 \int_{t_{\alpha}}^{\infty}\left(\frac{14}{16}\right)\left(\frac{1}{6} N_{\mathrm{A}}^{2} \rho^{2}\langle\sigma v\rangle_{3 \alpha}\right) d t,
$$

where $t_{\alpha}$ is defined by $T_{9 \alpha} \equiv T_{9 i} \exp \left(-t_{\alpha} / 3 \tau_{\mathrm{HD}}\right)$. Since $r_{3 \alpha}$ is fairly insensitive to the temperature for the high temperature regime under consideration one may take

$$
\begin{aligned}
\rho^{2} N_{\mathrm{A}}^{2}\langle\sigma v\rangle_{3 \alpha} & \simeq 1.3 \times 10^{-10} \rho^{2} \\
& =1.3 \times 10^{-10} \rho_{i}{ }^{2} \exp \left(-2 t / \tau_{\mathrm{HD}}\right),
\end{aligned}
$$

an approximation which is good to within 50 percent in the region $T_{9}=3.0$ to $T_{9}=8.0$. Then equation (68) may be integrated to yield

$$
X_{\alpha f}^{-2}=X_{\alpha}^{-2}\left(T_{9 \alpha}\right)+1.8 \times 10^{-11} \tau_{\mathrm{HD}} \rho_{i}^{2} \exp \left(-2 t_{\alpha} / \tau_{\mathrm{HD}}\right) .
$$

If $X_{\alpha}\left(T_{9 \alpha}\right)=0.01$ and $T_{9 \alpha}=5.0$, then for $\tau_{\mathrm{HD}}<0.15 \mathrm{~s}$ the above equation says that $X_{\alpha f} \simeq X_{\alpha}\left(T_{9 \alpha}\right)$ and merely meeting the requirement that $\rho$ at $T_{9 \alpha}=5.0$ is less than $6.0 \times 10^{7} \mathrm{~g} \mathrm{~cm}^{-3}$ guarantees an alpha-rich freeze-out. If $\tau_{\mathrm{HD}}$ exceeds this value then for an alpha-rich freeze-out one must have $X_{\alpha}\left(T_{9 \alpha}\right) \gg X_{\alpha f}$. Demanding $X_{\alpha f}$ be greater than 0.01 in either case leads to the condition on $\rho$ at $T_{9 \alpha}=5.0$ that

$$
\rho\left(T_{9}=5\right)<\min \left\{\begin{array}{l}
6 \times 10^{7} \\
2.4 \times 10^{7} \tau_{\mathrm{HD}}^{-1 / 2} .
\end{array}\right.
$$

Assuming a gravitational time scale for the expansion and equation (3) with $\gamma=4 / 3$, this result specifies that an alpha-rich freeze-out will occur only if the initial conditions of the evolution satisfy

$$
\rho_{i}<\min \left\{\begin{array}{l}
4.5 \times 10^{5} T_{9 i}{ }^{3} \\
2.5 \times 10^{5} T_{9 i}{ }^{4} \chi^{-2 / 3}
\end{array} .\right.
$$

The area delineated by this restriction was shown in figure 20 for $\chi=1$ and $\chi=5$. For $\chi=1$ the maximum density is always determined by the demand that $X_{\alpha}\left(T_{9 \alpha}\right)>$ 0.01. For $\chi=5$ and $T_{9 i}<7.0$, the additional constraint that $X_{\alpha}$ is not depleted in the course of freeze-out from $T_{9}=5.0$ requires a lower density than purely equilibrium considerations would predict. Due to the approximate manner in which equation (72) was derived it would not be surprising to find that the "changeover density" may vary as much as a factor of 2 from the predicted value. The ideas behind the derivation should, however, be generally valid. The predictions from this calculation are confirmed by examination of a variety of runs (see for example ATW).

The nucleosynthesis resulting from a typical evolution of this type is portrayed in figure 23. The species produced here are obviously different from the results of silicon burning. The species ${ }^{58} \mathrm{Ni}$ is now the dominant neutron-rich isotope produced. The species ${ }^{54} \mathrm{Fe}$ is not produced in significant amount. Most of the remainder of the material is bound up in the form of ${ }^{56} \mathrm{Ni}$ although there exists sufficient material in the form of zinc isotopes to account quite well for the solar-system abundances of other neutron-rich nickel isotopes. All in all, the species ${ }^{56} \mathrm{Fe},{ }^{58} \mathrm{Ni},{ }^{59} \mathrm{Co},{ }^{60} \mathrm{Ni},{ }^{61} \mathrm{Ni}$, and ${ }^{62} \mathrm{Ni}$ are produced in relative amounts which agree excellently with their solar-system values. Species beyond $A=62$ are produced in small amounts, though the values are far below the required solar-system values. The calculations are as yet unreliable for 


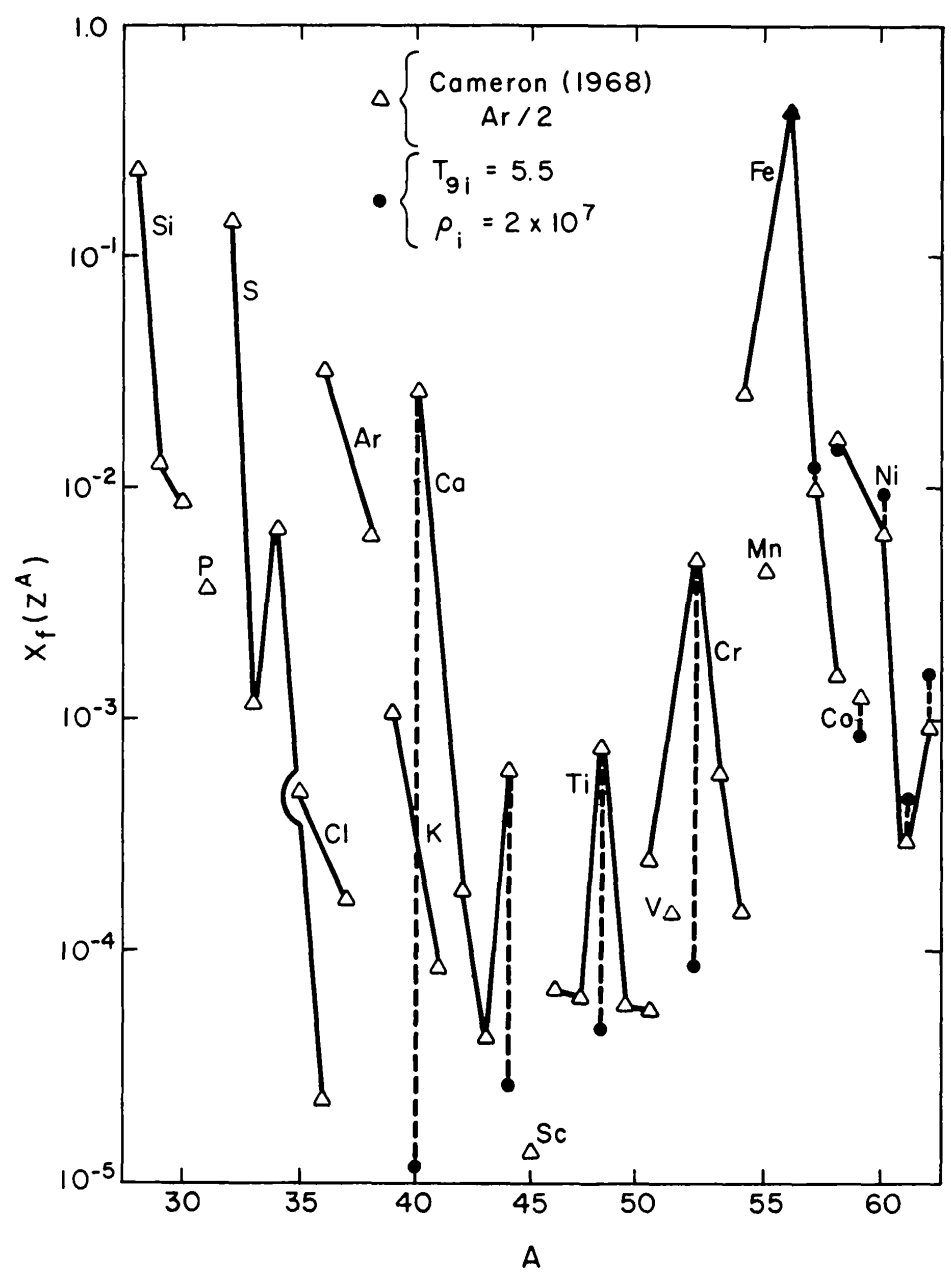

FIG. 23.-Final abundances resulting from an $e$-process with an alpha-rich freeze-out. The final abundances by mass fraction of the species $Z^{A}$ resulting from the explosive burning of composition "O" are shown compared to the solar abundance distribution. These results have been normalized to ${ }^{56} \mathrm{Fe}$. The initial conditions of the run were $T_{9 i}=5.5, \rho_{i}=2.0 \times 10^{7}, \chi=4.45$, and $\eta=$ $1.53 \times 10^{-3}$. The progenitors of these stable species are the elements listed in table 11 . Abundances having mass fractions less than $10^{-5}$ are not indicated. The final mass fraction of free $\alpha$-particles was $X_{\alpha}=0.022$.

these species. Also, production of such species would appear to be favored by still higher alpha densities at freeze-out and longer time scales for the expansion.

\section{c) The Correct Neutron Excess}

In figure 24 is shown a composite of the results of silicon burning and alpha-rich freeze-out for various values of $\eta$. This particular composite was formed by taking equal parts by mass of two runs both having $\rho_{i}=2.0 \times 10^{7}$ and $\chi=4.45$. One had an initial temperature of $T_{9 i}=4.7$, the other $T_{9 i}=5.5$. The former underwent silicon burning, the latter an alpha-rich freeze-out. The sum of the results has been normalized to ${ }^{56} \mathrm{Fe}$. From the resultant abundances, particularly those of the abundant neutron-rich isotopes ${ }^{54} \mathrm{Fe},{ }^{58} \mathrm{Ni}$, and ${ }^{62} \mathrm{Ni}$, it appears that an excellent fit to the solarsystem abundance distribution in the iron-peak region is obtained for $\eta$ approximately equal to 0.002 . Surely variation of $\eta$ of a factor of 2 in either direction from this value would result in severe over- or underproductions. Weak interactions in both of these runs were not effective in changing $\eta$ from its initial value. Thus again, as in the cases 


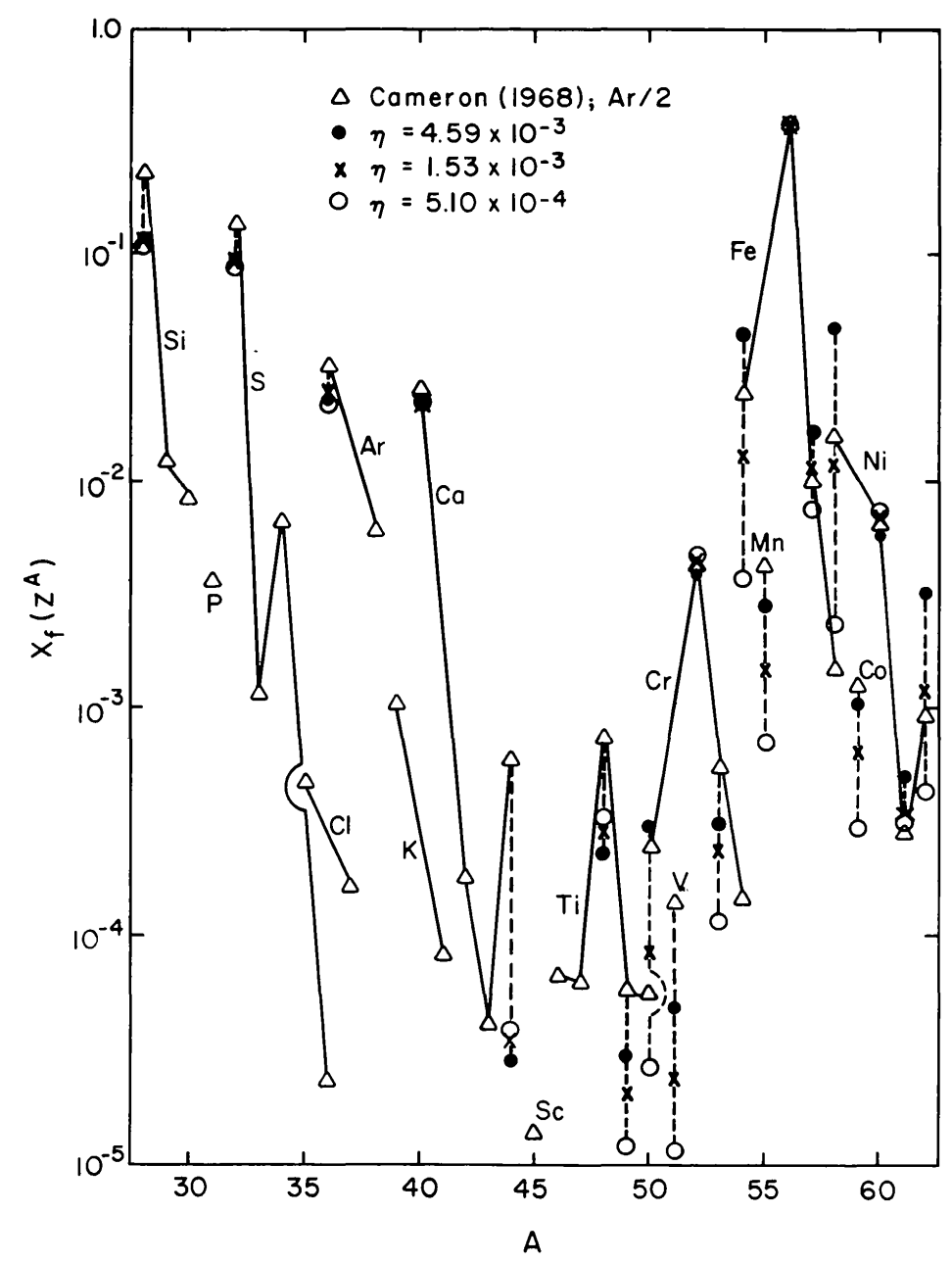

FIG. 24.-Variation of the final abundances with respect to the neutron excess. The final abundances by mass fraction of the species $Z^{A}$ are shown for a composite of two runs, both of which employed an initial density of $\rho_{i}=2.0 \times 10^{7}$ and time-scale parameter $\chi=4.45$. The temperature of the two runs were $T_{9 i}=4.7$ and $T_{9 i}=5.5$. The initial composition was composition "O." Three such composites were formed of the results of runs employing the three values of $\eta$ shown in the figure. The results, normalized to ${ }^{56} \mathrm{Fe}$, are shown compared to the solar abundance distribution. The best fit to many abundant species seems to occur for $\eta \sim 0.002$.

of explosive carbon burning and of explosive oxygen burning, it seems that the best value of $\eta$ to employ if the solar abundance distribution is to be correctly represented is $\eta \sim 0.002$. To a possible objection we note that other superpositions of siliconburning zones that give reasonable representations of the iron-peak abundances have very similar dependence on $\eta$, so the particular superposition chosen here is representative. This dependence can be summarized as follows: the abundances of final species whose primary progenitor is an $\alpha$-particle nucleus (e.g., ${ }^{40} \mathrm{Ca},{ }^{56} \mathrm{Ni}$ ) are essentially independent of $\eta$; those whose progenitors are alpha-plus- $2 n$ nuclei (e.g., ${ }^{54} \mathrm{Fe},{ }^{58} \mathrm{Ni}$ ) depend in an approximately linear way on $\eta$; and those whose progenitors are alphaplus- $n$ nuclei (e.g., ${ }^{53} \mathrm{Cr},{ }^{57} \mathrm{Ni}$ ) vary approximately as $\sqrt{ } \eta$. These relationships come about because the free-neutron density at freeze-out varies roughly as $\sqrt{ } \eta$ (Arnett 1971).

Mention was made in the introduction of this paper that a solution to the production of the iron peak of an entirely different nature has long been known. Most 
recently discussed by Fowler and Hoyle (1964) this solution proposes that the ironpeak species are produced in nuclear statistical equilibrium at $T_{9} \sim 3.8$ as themselves, not as the radioactive progenitors given in table 11 . This requires a much higher $\eta$, say $\eta$ approximately that of ${ }^{56} \mathrm{Fe}(\eta=0.071)$. An excellent fit to the iron isotopes, including ${ }^{58} \mathrm{Fe}$, results. However, problems with this type of solution have been encountered. First Clayton and Woosley (1969) have shown that any attempt to produce the iron isotopes in a single zone (i.e., one $\rho, T_{9}$, and $\eta$ ) in a nuclear equilibrium where ${ }^{56} \mathrm{Fe}$ is produced as ${ }^{56} \mathrm{Fe}$, not ${ }^{56} \mathrm{Ni}$, will always result in underproduction of ${ }^{58} \mathrm{Ni}$. This difficulty may be circumvented, however, if two zones are allowed to participate; ${ }^{54} \mathrm{Fe}$ and ${ }^{58} \mathrm{Ni}$ are produced together at an $\eta$ of about 0.037 and the remaining iron isotopes are coproduced in another zone again having an $\eta$ of 0.071 . The appropriate sum of two such zones can give an excellent fit to the iron isotopes as well as ${ }^{58} \mathrm{Ni}$. But it remains to be seen if solutions of this type can successfully avoid serious overproduction of other nuclei, especially ${ }^{52} \mathrm{Cr}$ and ${ }^{60} \mathrm{Ni}$. We will not discuss the "high- $\eta$ " $e$-processes here, because that is not really the subject of this work. However, we do believe it probable that the "low- $\eta$ " explosive solution, which (1) does produce most of the abundant species, (2) does not overproduce any species, and (3) is thermodynamically plausible, will prove to be the dominant astrophysical solution.

\section{CONCLUSIONS}

The nature of explosive oxygen and silicon burning has been investigated for a variety of plausible conditions. The results of the investigation show that a large fraction of the naturally occurring elements in the mass range $28 \leq A \leq 62$ can be produced by these processes in amounts consistent with their solar abundances and that the thermodynamic conditions leading to such a synthesis, though somewhat restricted, are not unique.

In figure 25 is shown a composite of the results of explosive oxygen burning, silicon burning, and an e-process with an alpha-rich freeze-out. This particular composite was formed by taking equal parts by mass of the results of these three types of explosive burning. The overall fit to the solar distribution is extremely good. All in all some 28 species are fit to within a factor of 3 of their solar abundance. The most abundant isotope of each element except titanium and phosphorus is in virtually exact agreement with the observed abundance. All elemental abundances (sum of the isotopes of a given $Z$ ), except phosphorus, are fit to within a factor of 3, and many isotopic ratios are correctly given. Most of the species that are not produced are either of low abundance or are synthesized in explosive carbon burning. In particular Arnett (1969b) found that ${ }^{29} \mathrm{Si},{ }^{30} \mathrm{Si}$, and ${ }^{31} \mathrm{P}$ are likely due to the explosive carbon burning, and Howard et al. (1972) found that ${ }^{36} \mathrm{~S},{ }^{40} \mathrm{Ar},{ }^{43} \mathrm{Ca},{ }^{46} \mathrm{Ca},{ }^{48} \mathrm{Ca},{ }^{45} \mathrm{Sc},{ }^{47} \mathrm{Ti},{ }^{49} \mathrm{Ti},{ }^{50} \mathrm{Ti}$, and several heavier nuclei may be in large part synthesized in the carbon explosion by secondary reactions that occur on heavy seed nuclei that are initially present in low abundance. Only the nuclei ${ }^{44} \mathrm{Ca},{ }^{54} \mathrm{Cr}$, and ${ }^{58} \mathrm{Fe}$ find no simple source in these explosions. Their origins will probably lie in a small amount of special ejecta enriched in these isotopes due to specific causes. The fact that the composite shown here was obtained by taking equal amounts of the results of explosive oxygen burning, silicon burning, and an $e$-process with an alpha-rich freeze-out suggests that roughly equal amounts by mass of the supernova may have been subjected to the conditions appropriate to the three types of burning. The conditions implied by these various burning processes have been discussed in detail in the pertinent sections. Basically the contention that the elements in the mass range $28 \leq A \leq 62$ were synthesized in such a manner rests on the fact that the 28 points represented in figure 25 were fitted by choosing values for only seven parameters (in effect, one value of $\eta$ and three pairs of initial temperatures and densities), all of which are astrophysically plausible. Such 


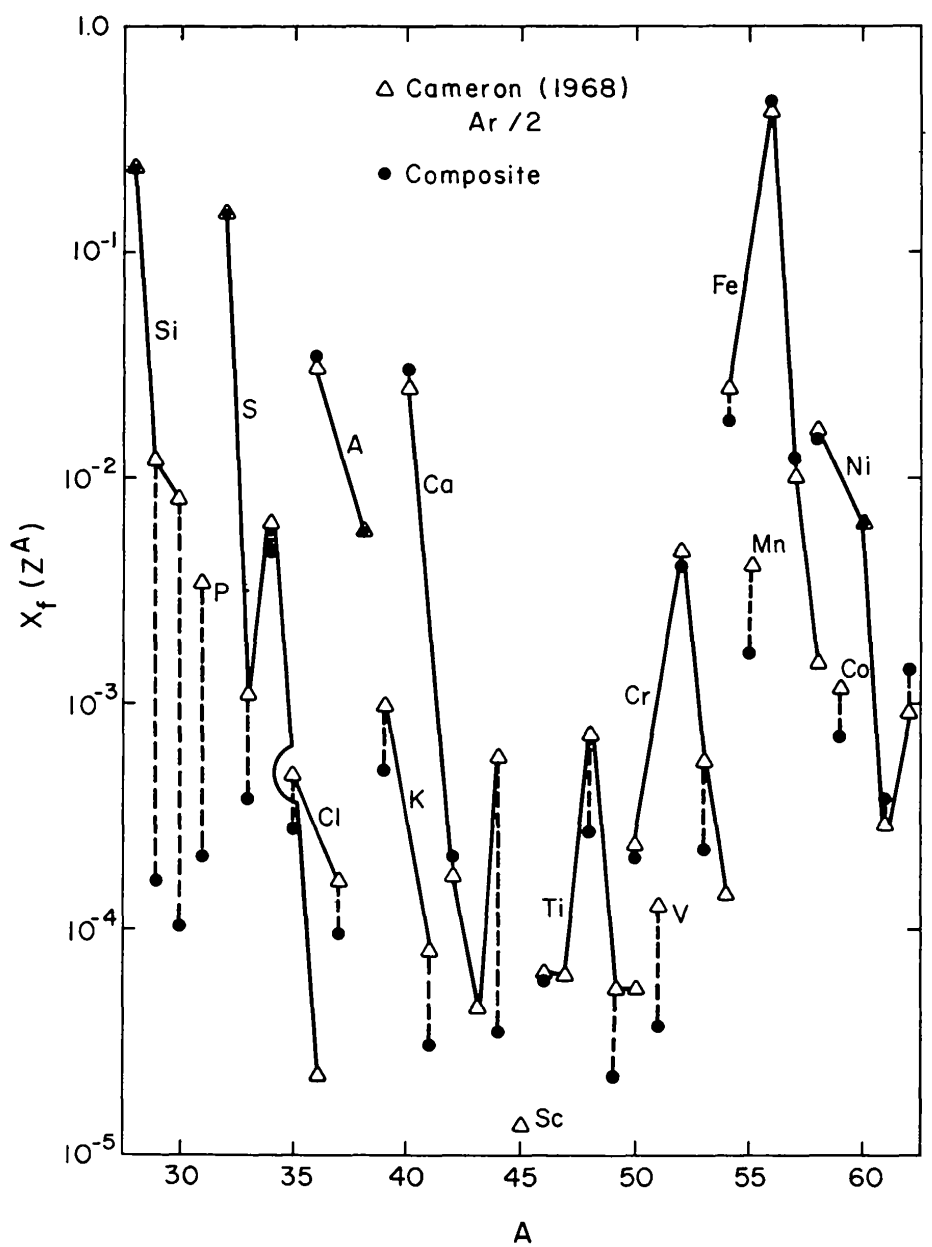

FIG. 25.-Composite of explosive oxygen and silicon burning results. The final abundances by mass fraction of the species $Z^{A}$ are shown for a composite of equal parts by mass of three types of nuclear evolution. All three runs employed composition "O" and $\eta=0.002$ : one run was the "standard" explosive-oxygen-burning run $\left(\rho_{i}=2.0 \times 10^{5}, T_{9 i}=3.6, \chi=1.0\right)$; one was silicon burning $\left(\rho_{i}=2.0 \times 10^{7}, T_{9 t}=4.7, \chi=4.45\right)$; one was an $e$-process with an alpha-rich freeze-out $\left(\rho_{i}=2.0 \times 10^{7}, T_{9 i}=5.5, x=4.45\right)$. The composite is shown compared to the observed solar abundance distribution. Results have been normalized to ${ }^{28} \mathrm{Si}$. Abundances less than $10^{-5}$ by mass fraction are not indicated.

agreement is strongly suggestive of at least a partial explanation of the origin of these elements, although several other superpositions could work as well as that leading to figure 25.

Even so, results of this type should be treated with caution, and care be paid to the following assumptions which underly not only figure 25 but, for the most part, all of this investigation.

1) It has been assumed that the solar-system abundance distribution is well representative of a "cosmic" abundance distribution; more specifically, that the solar composition inferred from a variety of measurements on solar-system objects is representative of the average composition of the interstellar medium. If it is, then either the elements have all been made concurrently and/or the mixing of the interstellar medium has occurred on a time scale that is short in comparison to the time for stars to form within fresh debris. Departure from this idealization could have distorted the solar abundance pattern in some significant way. 
We have also assumed that the abundance table of Cameron (1968) is, with reduction of Ar by a factor of 2, actually representative of solar-system abundances. New measurements of relevance continue to be made, however, and we call the reader's attention in this regard to Crawford et al. (1972). Their measurements of energetic heavy nuclei ejected by solar flares suggest that the Si-S-Ar triad is much more steeply falling in abundance than Cameron's values, and that $\mathrm{Ca}$ is probably more abundant than Ar. If this suggestion is correct, the conclusions to follow regarding the superposition of the various burning modes will require modification. It is therefore perhaps worth emphasizing again that it is the measurements of solar abundances that reveal the relative proportions of the several explosive-burning zones as they have apparently occurred in exploding stars. The theory of the stellar explosion does not yet give this information.

2) Even though mixing may have been effective, to argue that stars have exploded like the best-fitted explosions is to assume that all of the elements that are attributable to any one type of burning process, for instance the elements resulting from explosive oxygen burning, are always produced concurrently. Thus it is assumed, for example, that ${ }^{34} \mathrm{~S}$ and ${ }^{38} \mathrm{Ar}$ are always produced in amounts similar to their solar values, not a large amount of ${ }^{34} \mathrm{~S}$ in one star (or zone) and a large amount of ${ }^{38} \mathrm{Ar}$ in another. The conditions derived as appropriate to the various processes are to be considered as representative of the actual continuum of conditions that would in reality exist in the various zones of a typical supernova or the dominant zones of several types of supernovae.

3) It has been assumed that all nuclear cross-sections employed are at least approximately correct and that no important reaction link has been omitted. This assumption is not as critical as it may first appear to be due to the quasi-equilibrium nature of explosive oxygen and silicon burning. Critical cross-sections are noted with a dagger in table 13.

4) The expansion has been regarded as adiabatic with $\gamma \simeq 4 / 3$ and is assumed to occur on approximately a hydrodynamic time scale. Also, the initial compositions employed are accepted as representative. Where the effect may be large, the time scale and initial composition have been varied and the effects examined.

5) All solutions here have been of the "low- $\eta$ " variety. Variation of $\eta$ about 0.002 shows that $\eta=0.002-0.004$ is the best range to employ in both explosive oxygen and silicon burning in order to yield results that best match the solar distribution. However, the solution may not be either unique or complete. Equilibrium solutions to the iron-peak elements at high values of $\eta$, say $\eta \sim 0.07$, also yield reasonably good fits. Overproductions occur when all the iron-peak species are synthesized in such a fashion; however, an excellent fit to the iron-peak abundances may result from the mixing of a small amount of "high- $\eta$ " solution into a predominantly "low- $\eta$ " solution. This results in the production of the neutron-rich species ${ }^{50} \mathrm{Ti},{ }^{54} \mathrm{Cr}$, and ${ }^{58} \mathrm{Fe}$. Whether such a solution is astrophysically feasible or not remains to be determined.

It should be borne in mind that there exists overlap in the identity of the species synthesized in silicon burning, both with those synthesized in explosive oxygen burning and with those synthesized in the $e$-process. Oxygen burning synthesizes nuclei almost exclusively in the set $G_{1}(28 \leq A \leq 45)$, and the $e$-process synthesizes nuclei only in $G_{2}(46 \leq A \leq 66)$, whereas silicon burning synthesizes selected nuclei within both sets. Many species in both mass ranges may be synthesized by either or both of two processes, one of which is silicon burning. The processes leading to figure 25 are then somewhat ambiguous. All of the species that are produced in the mass range $28 \leq$ $A \leqslant 45$ in figure 25 could have been produced by explosive oxygen burning alone. Indeed, excessive production of the alpha nuclei ${ }^{28} \mathrm{Si},{ }^{32} \mathrm{~S},{ }^{36} \mathrm{Ar}$, and ${ }^{40} \mathrm{Ca}$ by silicon burning can render inconsistent many isotopic ratios, for example ${ }^{38} \mathrm{Ar} /{ }^{36} \mathrm{Ar}$, that are well reproduced by explosive oxygen burning. The contribution to the abundances of 
species in this mass range by silicon burning should therefore not be too large. No noticeable inconsistencies would result if the contribution were completely negligible. Moreover, most of the species produced by silicon burning in the iron-peak region could have been synthesized equally well by an $e$-process with a normal freeze-out (compare figs. 19 and 22). Thus the excellent fit to the solar abundances in figure 25 could just as well have been obtained by taking some suitable composite of the results of explosive oxygen burning and the two varieties of $e$-process freeze-out. A siliconburning freeze-out may not even be necessary to the production of any of the species in the $28 \leq A \leqslant 62$ region! Our " $e$-process" is essentially the old $e$-process solution to the synthesis of the iron peak except that the nuclear equilibrium here would be characterized by a low value of $\eta$ and two types of freeze-out (that is a high- and a low-density solution). The current uncertainties in our six assumptions, in the astrophysical models, and in the experimental determinations of the elemental abundances do not yet allow a choice to be made between silicon burning and an $e$-process with a normal freeze-out.

Michaud and Fowler (1972) introduced a somewhat different point of view that is relevant to this discussion. They attempt to account for the nucleosynthesis of all nuclei having $28 \leq A \leq 58$ by silicon burning alone. To achieve this they took advantage of the fact that the quasi-equilibrium abundances early in silicon burning are largely negligible in the set $G_{2}$ because of the slowness with which material penetrates the $A \simeq 45$ abundance minimum in the transfer of nuclei from $G_{1}$ to $G_{2}$. With the quasi-equilibrium largely constrained to $G_{1}$, the abundance pattern is very similar to that of the quasi-equilibrium during explosive oxygen burning. Thus they were able to achieve with their approximate treatment an abundance distribution similar to the solar one by integrating over a continuum of silicon burning conditions. In effect, they use the mass zones that burn very small fractions of the silicon to achieve the same ends that we achieve by explosive oxygen burning. Although their paper is quite sound as a model-dependent calculation, it has serious weaknesses as a primary explanation of the abundances, and we would be remiss if we did not point them out within the context of this paper: (1) The explosions of silicon cores should not, perhaps, be expected to account totally for the solar abundances because the overlying oxygen mass will surely also explode. This is an astrophysical question rather than a nuclear one. (2) The continuous range of imposed conditions chosen to burn between roughly 10 and 90 percent of the ${ }^{28} \mathrm{Si}$ may be quite unrealistic for actual dynamic models. Silicon burning alone cannot succeed without proscribing its range of conditions closely. (3) The abundances produced are not really a good fit. Except for carefully chosen conditions, there is a strong tendency to overproduce ${ }^{50} \mathrm{Cr},{ }^{54} \mathrm{Fe}$, and ${ }^{55} \mathrm{Mn}$ in their approach, whereas ${ }^{38} \mathrm{Ar}$ is unavoidably underproduced. The basic reason for this is shown clearly by our network integrations and has been known for some time (e.g., TA70). By the time alpha nuclei like ${ }^{36} \mathrm{Ar}$ and ${ }^{40} \mathrm{Ca}$ have risen to even one-tenth of their solar values relative to ${ }^{28} \mathrm{Si}$, the neutron excess in this region is siphoned from $G_{1}$ into $G_{2}$ by flows through the bottleneck resulting in large abundances of ${ }^{50} \mathrm{Cr}$ and ${ }^{54} \mathrm{Fe}$ and small abundances of ${ }^{38} \mathrm{Ar}$. Other difficulties of similar type occur. In explosive oxygen burning, on the other hand, the quickly burning oxygen fuel is able to build up the abundances of the alpha nuclei up to ${ }^{40} \mathrm{Ca}$ before significant flow from $G_{1}$ into $G_{2}$ can occur. For this reason, explosive oxygen burning can synthesize the nuclei in $G_{1}$ considerably more naturally and successfully than can explosive silicon burning. Although we do not believe that the Michaud and Fowler scenario is the appropriate one for nucleosynthesis, it seems that any judgment should await dynamic models of exploding evolved stars. It should be noted, however, that if the Si-S-Ar triad is more rapidly falling in abundance as suggested by Crawford $e t$ al. (1972), then the difficulty of Michaud and Fowler's scenario is less severe with regard to the underproduction of ${ }^{38} \mathrm{Ar}$. 
Exploding models will be necessary before the relevance of this entire paper is clear. Despite the great success of these explosions in reproducing the observed abundances of the nuclear species, no real understanding will exist until the explosions themselves are understood. In the meantime we submit this work as a compendium of much of the knowledge we have achieved in nuclear astrophysics.

We are indebted to many people, but especially to Professors William A. Fowler and James Truran for their continuous assistance and to Professor Fred Hoyle and the Institute of Theoretical Astronomy, Cambridge, England for their hospitality and a generous grant of computer time while this paper was in preparation. This research has been supported by the National Science Foundation grants GP-18335 and GP-23459.

\section{APPENDIX \\ THERMONUCLEAR REACTION RATES}

During the course of this investigation it has been necessary to draw heavily upon current nuclear-data analysis. In particular, values for a great variety of nuclearreaction rates are required for the proper coupling of the various networks which have been employed in these calculations. Sample values of the rates actually employed are given for two different temperatures in table 13. Rates which we have determined to have no significant importance during explosive oxygen and silicon burning have been omitted. The formulae used to generate these rates are, in general, available in the reference cited, although many have been updated by private communication. For more detailed information regarding the exact temperature dependence of the rate and energy dependence of the cross-section, the interested reader should consult the source.

In many cases the rates used here are based upon fittings to experimental data or the extrapolation of such fittings. The "effective energy" for a given reaction between two particles of charge $Z_{1}$ and $Z_{2}$ with reduced mass $A$ is approximately given by (Fowler and Hoyle 1964)

$$
E_{0}=0.122\left(Z_{1}{ }^{2} Z_{2}{ }^{2} A T_{9}{ }^{2}\right)^{1 / 3} \mathrm{MeV} .
$$

It is at this center-of-mass energy that the majority of the reactions between species 1 and 2 can be expected to take place. For most of the charged-particle reactions of interest in oxygen and silicon burning $E_{0}$ will have values on the order of 1-10 MeV. These energies are much larger than characteristic energies for hydrogen and helium burning. Thus, large extrapolations of the cross-section to lower energies than can be measured in the laboratory are generally not required. We therefore believe the errors in all of the rates listed in table 13 with a reference code number (2) to be quite small, probably no more than a factor of 2 in the temperature range of interest. In addition, the reaction rates for ${ }^{12} \mathrm{C}+{ }^{12} \mathrm{C},{ }^{16} \mathrm{O}+{ }^{16} \mathrm{O}$, and ${ }^{12} \mathrm{C}+{ }^{16} \mathrm{O}$ have experimental determinations which render them fairly reliable. The first two are probably accurate to about 50 percent, the latter, by a factor of 2 . The triple-alpha rate is that of Fowler, Caughlan, and Zimmerman (1967), updated by more accurate measurements of the energy of the second excited state in the ${ }^{12} \mathrm{C}$ compound nucleus by Austin, Trentelman, and Kashy (1971). Since most reactions in the temperature range of our interest (3.0 $\left.\leq T_{9} \leq 5.0\right)$ occur through the 7.66-MeV excited state rather than higher excited states, we may also consider this rate to be accurate to about 50 percent.

Where experimental data is not available the semiempirical formulae of Truran et al. (1966) updated by private communication (Truran 1971) have been employed. This includes almost all $(n, \gamma),(p, n)$, and $(\alpha, n)$ rates as well as many of the rates for 
$(p, \gamma),(\alpha, p)$, and $(\alpha, \gamma)$ reactions. All weak-interaction rates are taken from Hansen (1968). Both Truran's and Hansen's rates may be in error by factors of up to about 5, with a factor of 3 being typical.

Although improved values for all the cross-sections listed in table 13 would be, in principle, a desirable goal, the dominant properties of oxygen and silicon burning are in reality dependent upon a much smaller subset of nuclear-reaction information. The importance of a great many reaction rates is diminished by the tendency of the reaction links to approach equilibrium. In such cases the nuclear evolution is often much more sensitive to the (accurately known) binding energy of the species than to the actual value of a given cross-section. For such links the size of the cross-section only matters after the link has dropped out of equilibrium. Since the time remaining before freezeout is usually so small (except in the case of the particle-rich $e$-process) that the abundances involved change very little, the small change may often be treated as a perturbation or freeze-out correction. These corrections then contain the local crosssection dependence of the species. There are, however, several rates which are critical to this study. These are the rates which govern particle production or equilibration time scales and do not generally approach a state of equilibrium during the process considered. They are diacritically denoted in table 13 . These are the reactions for which we would be most interested in obtaining accurate measurements.

Corrections to rates due to electron screening and excited nuclear states have been ignored. Sample calculations using the formulae of Salpeter and Van Horn (1969) have shown that corrections due to electron screening should only be marginal (to within the accuracy of the cross-section employed) in the temperature-density range of interest. The effect of excited states present in the target and product nuclei should be included in a calculation of this type. Unfortunately, rate formulae which properly include the presence of excited states have not yet been generated, although such an effort is currently under way.

A rough estimate of the effect of including excited states in these calculations can be obtained by an examination of the nuclear partition functions for the species which we produce (see fig. 25 and table 11.). Consider the abundances of these species at the representative temperature $T_{9}=3.2$. A state of equilibrium will normally exist for species heavier than ${ }^{28} \mathrm{Si}$ (either in the restricted sense for explosive oxygen burning or total quasi-equilibrium for explosive silicon burning). Now the species which have the largest abundances in our calculations, e.g., ${ }^{28} \mathrm{Si},{ }^{32},{ }^{34} \mathrm{~S},{ }^{36},{ }^{38} \mathrm{Ar},{ }^{40} \mathrm{Ca},{ }^{52,}{ }^{54} \mathrm{Fe}$, and ${ }^{56,58} \mathrm{Ni}$ all have high first excited states $\left(E_{i} \geqslant 1.4 \mathrm{MeV}\right)$ and hence are affected very little by excited-state contributions at this temperature. Furthermore, these species contain almost all of the mass and excess neutrons and are dominating the determination of the free-particle abundances. Under these conditions we then expect that the abundances of species which do vary when excited states are introduced, for example, ${ }^{46} \mathrm{Ti},{ }^{48,}{ }^{49},{ }^{50} \mathrm{Cr},{ }^{51} \mathrm{Mn}$, etc., will change roughly as the ratio $G\left(T_{9}=3.2\right) / W_{0}$ where $G\left(T_{9}=3.2\right)$ is the nuclear partition function including the excited states and $W_{0}$ is the ground-state partition function used in these calculations. The introduction of excited states always acts to increase the abundance of the (low-abundance) species in question. We have calculated this fractional increase for all species that are produced in amounts comparable to their Cameron (1968) abundances by explosive oxygen and silicon burning and find that it is small. This statement applies, where appropriate, to the proton-rich progenitor of the species which is ultimately produced. For all such species lighter than $A=43$ the increase at $T_{9}=3.2$ is less than three percent; for species heavier than $A=43$ only ${ }^{46} \mathrm{Ti},{ }^{49},{ }^{50} \mathrm{Cr},{ }^{51} \mathrm{Mn},{ }^{52} \mathrm{Fe}$, and perhaps ${ }^{61} \mathrm{Zn}$ (for which no excited-state information is available) are changed by more than 20 percent. The maximum error is for ${ }^{51} \mathrm{Mn}$ (produces ${ }^{51} \mathrm{~V}$ ) which increases 58 percent. In some cases the small effect reflects the fact that an otherwise sensitive species is made as a proton-rich progenitor. For example, if ${ }^{57} \mathrm{Fe}$ were created as ${ }^{57} \mathrm{Fe}$ rather than ${ }^{57} \mathrm{Ni}$ the 
TABLE 13

IMPORTANT REACTION RATES*

\begin{tabular}{|c|c|c|c|c|c|c|}
\hline \multirow{3}{*}{ Target } & \multirow{3}{*}{ Reaction } & \multicolumn{4}{|c|}{$\mathrm{N}_{\mathrm{A}}\langle\sigma \mathrm{v}\rangle$} & \multirow{3}{*}{ Ref. } \\
\hline & & \multicolumn{2}{|c|}{ Forward Rate } & \multicolumn{2}{|c|}{ Reverse Rate } & \\
\hline & & $T_{9}=3.5$ & $\mathbf{T}_{9}=4.5$ & $T_{9}=3.5$ & $T_{9}=4.5$ & \\
\hline$\alpha$ & $3 \alpha+$ & $2.4(-11)$ & $1.7(-11)$ & $4.2(1)$ & $1.3(4)$ & 1,2 \\
\hline \multirow[t]{6}{*}{${ }^{12} \mathrm{c}$} & ${ }^{12} c+{ }^{12} c+$ & $5.9(0)$ & $2.8(2)$ & $\cdots$ & $\cdots$ & 1,3 \\
\hline & ${ }^{12} \mathrm{c}+{ }^{16} 0+$ & $5.0(-2)$ & $4.4(0)$ & $\cdots$ & $\cdots$ & 1,4 \\
\hline & $(n, Y)$ & $4.5(2)$ & $4.5(2)$ & $2.0(6)$ & $1.1(8)$ & 5 \\
\hline & $(p, \gamma)$ & $4.2(3)$ & $4.2(3)$ & $3.9(11)$ & $2.4(12)$ & 2 \\
\hline & $(\alpha, p)$ & $2.0(0)$ & $1.2(2)$ & $3.9(7)$ & $5.9(7)$ & 2 \\
\hline & $(\alpha, \gamma) \dagger$ & $1.8(-1)$ & $9.9(-1)$ & $2.9(0)$ & $4.6(3)$ & 2 \\
\hline \multirow[t]{2}{*}{${ }^{13} \mathrm{C}$} & $(p, \gamma)$ & $4.6(4)$ & $5.1(4)$ & $4.8(4)$ & $2.0(7)$ & 2 \\
\hline & $(\alpha, n)+$ & $1.6(5)$ & $2.2(5)$ & $5.8(2)$ & $4.2(3)$ & 2 \\
\hline $13{ }_{N}$ & $(\alpha, p)+$ & $1.7(6)$ & $9.7(6)$ & $3.1(-1)$ & $8.1(1)$ & 5 \\
\hline \multirow[t]{2}{*}{$14 \mathrm{~N}$} & $(\alpha, p)$ & $5.9(4)$ & $2.4(5)$ & $4.5(6)$ & $7.6(6)$ & 2 \\
\hline & $(\alpha, \gamma)$ & $2.6(2)$ & $5.5(2)$ & $4.1(7)$ & $3.2(9)$ & 2 \\
\hline \multirow[t]{2}{*}{${ }^{15}{ }_{\mathrm{N}}$} & $(p, y)+$ & $5.1(4)$ & $6.9(4)$ & $4.1(-2)$ & $6.2(2)$ & 2 \\
\hline & $(\alpha, \gamma)$ & $1.4(1)$ & $2.3(1)$ & $8.3(6)$ & $3.9(8)$ & 5 \\
\hline \multirow[t]{4}{*}{${ }^{16} 0$} & ${ }^{16} 0+{ }^{16} 0+$ & $1.7(-4)$ & $8.4(-2)$ & $\cdots$ & & 1,6 \\
\hline & $(n, y)$ & $4.2(3)$ & $4.0(3)$ & $9.0(7)$ & $2.7(9)$ & 5 \\
\hline & $(\alpha, p)$ & $5.0(-5)$ & $2.3(-2)$ & $3.7(7)$ & $4.3(7)$ & 2 \\
\hline & $(\alpha, \gamma) \dagger$ & $2.3(0)$ & $3.7(0)$ & $1.3(5)$ & $1.0(7)$ & 2 \\
\hline \multirow[t]{2}{*}{${ }^{17} 0$} & $(p, \gamma)$ & $1.6(4)$ & $2.8(4)$ & $3.3(7)$ & $5.0(9)$ & 2 \\
\hline & $(\alpha, n)$ & $9.8(5)$ & $6.8(6)$ & $2.6(6)$ & $2.8(7)$ & 5 \\
\hline${ }^{18}{ }_{F}$ & $(\alpha, p)$ & $4.3(5)$ & $3.6(6)$ & $3.2(3)$ & $9.7(4)$ & 5 \\
\hline \multirow[t]{4}{*}{$20 \mathrm{Ne}$} & $(p, \gamma)$ & $7.6(3)$ & $7.4(3)$ & $7.3(10)$ & $6.2(11)$ & 2 \\
\hline & $(\alpha, p) \dagger$ & $4.9(2)$ & $4.3(3)$ & $1.0(6)$ & $1.6(6)$ & 2 \\
\hline & $(\alpha, n)$ & $5.6(-3)$ & $1.4(0)$ & $1.1(8)$ & $1.4(8)$ & 7 \\
\hline & $(\alpha, \gamma)+$ & $1.0(2)$ & $2.8(2)$ & $1.6(0)$ & $5.9(3)$ & 2 \\
\hline \multirow[t]{2}{*}{${ }^{21} \mathrm{Ne}$} & $(p, n)$ & $2.1(2)$ & $5.1(3)$ & $3.6(8)$ & $3.6(8)$ & 5 \\
\hline & $(\alpha, n)$ & $2.3(5)$ & $2.3(6)$ & $6.1(2)$ & $4.1(4)$ & 5 \\
\hline \multirow[t]{2}{*}{$22 \mathrm{Ne}$} & $(p, \gamma)$ & $7.0(4)$ & $8.4(4)$ & $4.7(2)$ & $5.3(5)$ & 5 \\
\hline & $(\alpha, n)$ & $2.5(5)$ & $2.5(6)$ & $6.2(5)$ & $4.4(6)$ & 7 \\
\hline${ }^{21}{ }_{\mathrm{Na}}$ & $(\alpha, p)$ & $9.5(4)$ & $1.1(6)$ & $1.5(-4)$ & $2.9(-1)$ & 5 \\
\hline \multirow[t]{5}{*}{${ }^{23} \mathrm{Na}$} & $(p, n)$ & $2.9(1)$ & $1.3(3)$ & $2.7(8)$ & $3.3(8)$ & 7 \\
\hline & $(p, \gamma)+$ & $1.8(4)$ & $2.2(4)$ & $1.3(-1)$ & $1.3(3)$ & 2 \\
\hline & $(\alpha, p)$ & $8.3(2)$ & $2.9(3)$ & $2.6(1)$ & $3.5(2)$ & 2 \\
\hline & $(\alpha, n)$ & $3.7(2)$ & $5.2(3)$ & $9.1(7)$ & $1.4(8)$ & 7 \\
\hline & $(\alpha, \gamma)$ & $4.4(1)$ & $2.1(2)$ & $3.4(-2)$ & $4.0(2)$ & 7 \\
\hline \multirow[t]{4}{*}{${ }^{24} \mathrm{Mg}$} & $(n, \gamma)$ & $1.6(5)$ & $1.9(5)$ & $8.8(4)$ & $3.5(7)$ & 7 \\
\hline & $(\alpha, p)+$ & $1.5(3)$ & $9.1(3)$ & $1.7(5)$ & $3.1(5)$ & 2 \\
\hline & $(\alpha, n)$ & $6.5(-3)$ & $1.5(0)$ & $8.4(7)$ & $9.9(7)$ & 7 \\
\hline & $(\alpha, y)+$ & $2.4(1)$ & $6.4(1)$ & $4.2(-2)$ & $2.5(2)$ & 2 \\
\hline \multirow[t]{4}{*}{${ }^{25} \mathrm{Mg}$} & $(p, \gamma)$ & $1.6(4)$ & $2.3(4)$ & $9.7(6)$ & $2.1(9)$ & 2 \\
\hline & $(\alpha, p)$ & $5.4(2)$ & $5.9(3)$ & $5.9(5)$ & $2.6(6)$ & 7 \\
\hline & $(\alpha, n)$ & $1.9(3)$ & $1.4(4)$ & $5.9(0)$ & $2.9(2)$ & 7 \\
\hline & $(\alpha, \gamma)$ & $3.5(0)$ & $2.6(1)$ & $4.0(-4)$ & $1.6(1)$ & 7 \\
\hline \multirow[t]{3}{*}{${ }^{26} \mathrm{Mg}$} & $(p, n)$ & $2.3(1)$ & $9.0(2)$ & $1.8(8)$ & $2.1(8)$ & 7 \\
\hline & $(p, \gamma)$ & $2.0(4)$ & $2.5(4)$ & $5.0(2)$ & $4.1(5)$ & 2 \\
\hline & $(\alpha, n)+$ & $2.8(3)$ & $2.2(4)$ & $4.3(3)$ & $3.4(4)$ & 7 \\
\hline
\end{tabular}


TABLE 13-Continued

\begin{tabular}{|c|c|c|c|c|c|c|}
\hline \multirow{3}{*}{ Target } & \multirow{3}{*}{ Reaction } & \multicolumn{4}{|c|}{$\mathrm{N}_{\mathrm{A}}\langle\sigma \mathrm{v}\rangle$} & \multirow{3}{*}{ Ref. } \\
\hline & & \multicolumn{2}{|c|}{ Forward Rate } & \multicolumn{2}{|c|}{ Reverse Rate } & \\
\hline & & $\mathrm{T}_{9}=3.5$ & $T_{9}=4.5$ & $\mathrm{~T}_{9}=3.5$ & $\mathrm{~T}_{9}=4.5$ & \\
\hline${ }^{26} \mathrm{AI}$ & $(\alpha, p)$ & $5.3(3)$ & $4.2(4)$ & $1.0(-3)$ & $2.8(-1)$ & 7 \\
\hline${ }^{27} \mathrm{Al}$ & $\begin{array}{l}(n, \gamma) \\
(p, n) \\
(p, \gamma)+ \\
(\alpha, p) \\
(\alpha, n) \\
(\alpha, \gamma)\end{array}$ & $\begin{array}{l}3.0(5) \\
3.6(0) \\
1.3(4) \\
3.4(2) \\
2.2(2) \\
8.5(0)\end{array}$ & $\begin{array}{l}3.7(5) \\
2.5(2) \\
2.0(4) \\
1.4(3) \\
2.9(3) \\
5.8(1)\end{array}$ & $\begin{array}{l}1.6(6) \\
4.1(8) \\
2.1(-1) \\
2.6(0) \\
9.6(6) \\
1.3(-1)\end{array}$ & $\begin{array}{l}8.6(8) \\
4.6(8) \\
2.3(3) \\
6.2(1) \\
1.8(7) \\
1.6(3)\end{array}$ & $\begin{array}{l}7 \\
7 \\
2 \\
2 \\
7 \\
7\end{array}$ \\
\hline${ }^{28}{ }_{\mathrm{Al}}$ & $(p, n)$ & $1.7(7)$ & $3.8(7)$ & $4.8(1)$ & $1.9(3)$ & 7 \\
\hline${ }^{27} \mathrm{si}$ & $\begin{array}{l}(n, \gamma) \\
(\alpha, p)\end{array}$ & $\begin{array}{l}1.4\left(\begin{array}{l}5 \\
5.6( \\
0)\end{array}\right.\end{array}$ & $\begin{array}{l}1.8(5) \\
1.3(2)\end{array}$ & $\begin{array}{l}1.9(-8) \\
2.2(-3)\end{array}$ & $\begin{array}{l}1.1(-2) \\
4.6(-1)\end{array}$ & 7 \\
\hline${ }^{28} \mathrm{Si}$ & $\begin{array}{l}(n, \gamma) \\
(\alpha, p)+ \\
(\alpha, n) \\
(\alpha, y)+\end{array}$ & $\begin{array}{l}2.0(5) \\
2.6(2) \\
1.2(-4) \\
4.3(0)\end{array}$ & $\begin{array}{l}2.5(5) \\
2.0(3) \\
5.4(-2) \\
1.4(1)\end{array}$ & $\begin{array}{l}7.6(3) \\
2.5(5) \\
1.1(8) \\
1.8(2)\end{array}$ & $\begin{array}{l}7.0(6) \\
4.8(5) \\
1.3(8) \\
1.5(5)\end{array}$ & $\begin{array}{l}7 \\
2 \\
7 \\
2\end{array}$ \\
\hline${ }^{29} \mathrm{Si}$ & $\begin{array}{l}(n, \gamma) \\
(p, \gamma) \\
(\alpha, p) \\
(\alpha, n) \\
(\alpha, \gamma)\end{array}$ & $\begin{array}{l}2.9(5) \\
4.4(3) \\
1.7(1) \\
4.8(2) \\
3.2(0)\end{array}$ & $\begin{array}{l}3.5(5) \\
5.5(3) \\
4.0(2) \\
4.8(3) \\
2.4(1)\end{array}$ & $\begin{array}{l}3.7(1) \\
3.2(6) \\
1.3(5) \\
5.3(5) \\
3.8(1)\end{array}$ & $\begin{array}{l}1.6(5) \\
3.6(8) \\
5.2(5) \\
1.7(6) \\
8.1(4)\end{array}$ & $\begin{array}{l}7 \\
2 \\
7 \\
7 \\
7\end{array}$ \\
\hline${ }^{30} \mathrm{Si}$ & $\begin{array}{l}(p, n) \\
(p, \gamma)+ \\
(\alpha, p) \\
(\alpha, n) \\
(\alpha, \gamma)\end{array}$ & $\begin{array}{l}4.7(1) \\
1.4(4) \\
5.1(0) \\
1.7(2) \\
1.1(1)\end{array}$ & $\begin{array}{l}2.2(3) \\
1.9(4) \\
1.3(2) \\
3.2(3) \\
5.1(1)\end{array}$ & $\begin{array}{l}2.7(8) \\
2.7(4) \\
1.7(5) \\
1.6(7) \\
1.9(1)\end{array}$ & $\begin{array}{l}3.1(8) \\
1.2(7) \\
4.9(5) \\
2.4(7) \\
4.3(4)\end{array}$ & $\begin{array}{l}7 \\
2 \\
7 \\
7 \\
2\end{array}$ \\
\hline${ }^{30} \mathrm{P}$ & $\begin{array}{l}(p, \gamma) \\
(\alpha, p)\end{array}$ & $\begin{array}{l}7.8(3) \\
2.2(2)\end{array}$ & $\begin{array}{l}1.3(4) \\
2.6(3)\end{array}$ & $\begin{array}{l}2.5(6) \\
3.7(0)\end{array}$ & $\begin{array}{l}5.3(8) \\
1.3(2)\end{array}$ & $\begin{array}{l}7 \\
7\end{array}$ \\
\hline${ }^{31} \mathrm{P}$ & $\begin{array}{l}(n, \gamma) \\
(p, n) \\
(p, y)+ \\
(\alpha, p) \\
(\alpha, \gamma)\end{array}$ & $\begin{array}{l}2.9(5) \\
4.1(-1) \\
3.5(3) \\
4.2(1) \\
8.5(-1)\end{array}$ & $\begin{array}{l}3.4(5) \\
4.6(1) \\
5.0(3) \\
2.1(2) \\
9.1(0)\end{array}$ & $\begin{array}{l}8.7(4) \\
3.9(8) \\
1.5(2) \\
3.5(1) \\
1.5(1)\end{array}$ & $\begin{array}{l}5.2(7) \\
4.4(8) \\
2.1(5) \\
2.9(2) \\
4.1(4)\end{array}$ & $\begin{array}{l}7 \\
7 \\
2 \\
2 \\
7\end{array}$ \\
\hline${ }^{32} \mathrm{~s}$ & $\begin{array}{l}(n, \gamma) \\
(\alpha, p) \\
(\alpha, \gamma)\end{array}$ & $\begin{array}{l}6.2(5) \\
2.4(2) \\
3.7(-1)\end{array}$ & $\begin{array}{l}7.0(5) \\
2.1(3) \\
1.9(0)\end{array}$ & $\begin{array}{l}6.8(3) \\
1.0(5) \\
4.4(1)\end{array}$ & $\begin{array}{l}6.6(6) \\
2.2(5) \\
4.2(4)\end{array}$ & $\begin{array}{l}7 \\
2 \\
2\end{array}$ \\
\hline${ }^{33} \mathrm{~s}$ & $\begin{array}{l}(n, \gamma) \\
(\alpha, p) \\
(\alpha, n) \\
(\alpha, \gamma)\end{array}$ & $\begin{array}{l}4.6(5) \\
2.6(1) \\
5.7(1) \\
1.9(0)\end{array}$ & $\begin{array}{l}5.1(5) \\
4.9(2) \\
7.3(2) \\
1.3(1)\end{array}$ & $\begin{array}{l}8.0(0) \\
4.4(4) \\
6.0(5) \\
1.3(2)\end{array}$ & $\begin{array}{l}5.8(4) \\
2.0(5) \\
1.8(6) \\
2.1(5)\end{array}$ & $\begin{array}{l}7 \\
7 \\
7 \\
7\end{array}$ \\
\hline${ }^{34} \mathrm{~s}$ & $\begin{array}{l}(n, y) \\
(p, \gamma) \\
(\alpha, p) \\
(\alpha, n) \\
(\alpha, \gamma)\end{array}$ & $\begin{array}{l}1.9(5) \\
3.3(3) \\
8.6(0) \\
8.7(0) \\
9.6(0)\end{array}$ & $\begin{array}{l}2.4(5) \\
5.3(3) \\
1.6(2) \\
3.5(2) \\
4.9(1)\end{array}$ & $\begin{array}{l}5.1(5) \\
6.9(4) \\
1.8(5) \\
3.6(7) \\
1.7(2)\end{array}$ & $\begin{array}{l}1.6(8) \\
1.8(7) \\
3.6(5) \\
4.7(7) \\
2.6(5)\end{array}$ & $\begin{array}{l}7 \\
2 \\
2 \\
7 \\
2\end{array}$ \\
\hline${ }^{35} \mathrm{~s}$ & $\begin{array}{l}(p, n) \\
(p, \gamma)\end{array}$ & $\begin{array}{l}1.2(6) \\
1.7(3)\end{array}$ & $\begin{array}{l}3.6(6) \\
5.1(3)\end{array}$ & $\begin{array}{l}9.5(6) \\
5.8(2)\end{array}$ & $\begin{array}{l}1.8(7) \\
8.9(5)\end{array}$ & $\begin{array}{l}7 \\
7\end{array}$ \\
\hline${ }^{35} \mathrm{Cl}$ & $\begin{array}{l}(n, \gamma) \\
(p, \gamma) \\
(\alpha, p) \\
(\alpha, \gamma)\end{array}$ & $\begin{array}{l}9.4(5) \\
4.3(3) \\
4.4(1) \\
9.8(-1)\end{array}$ & $\begin{array}{l}1.1(6) \\
6.3(3) \\
5.8(2) \\
1.0(1)\end{array}$ & $\begin{array}{l}4.1(4) \\
1.2(3) \\
3.8(1) \\
1.7(1)\end{array}$ & $\begin{array}{l}3.9(7) \\
1.4(6) \\
9.4(2) \\
5.4(4)\end{array}$ & $\begin{array}{l}7 \\
2 \\
7 \\
7\end{array}$ \\
\hline${ }^{36} \mathrm{Cl}$ & $\begin{array}{l}(p, n) \\
(p, \gamma) \\
(\alpha, n)\end{array}$ & $\begin{array}{l}6.7(5) \\
3.7(3) \\
4.3(1)\end{array}$ & $\begin{array}{l}1.9(6) \\
8.2(3) \\
6.2(2)\end{array}$ & $\begin{array}{l}4.2(6) \\
1.6(2) \\
1.7(4)\end{array}$ & $\begin{array}{l}1.1(7) \\
3.2(5) \\
9.3(4)\end{array}$ & $\begin{array}{l}7 \\
7 \\
7\end{array}$ \\
\hline
\end{tabular}

306 
TABLE 13-Continued

\begin{tabular}{|c|c|c|c|c|c|c|}
\hline \multirow{3}{*}{ Target } & \multirow{3}{*}{ Reaction } & \multicolumn{4}{|c|}{$N_{A}\langle\sigma v\rangle$} & \multirow{3}{*}{ Ref. } \\
\hline & & \multicolumn{2}{|c|}{ Forward Rate } & \multicolumn{2}{|c|}{ Reverse Rate } & \\
\hline & & $\mathrm{T}_{9}=3.5$ & $T_{9}=4.5$ & $T_{9}=3.5$ & $T_{9}=4.5$ & \\
\hline${ }^{37} \mathrm{Cl}$ & $\begin{array}{l}(p, n) \\
(p, y)\end{array}$ & $\begin{array}{l}3.8(5) \\
2.4(4)\end{array}$ & $\begin{array}{l}1.6(6) \\
4.3(4)\end{array}$ & $\begin{array}{l}7.5(7) \\
2.2(1)\end{array}$ & $\begin{array}{l}1.0\left(\begin{array}{l}8 \\
1.1( \\
5)\end{array}\right.\end{array}$ & $\begin{array}{l}7 \\
2\end{array}$ \\
\hline${ }^{36} \mathrm{Ar}$ & $\begin{array}{l}(n, \gamma) \\
(\alpha, p) \\
(\alpha, \gamma)\end{array}$ & $\begin{array}{l}3.9(5) \\
2.0(1) \\
2.9(-2)\end{array}$ & $\begin{array}{l}4.5(5) \\
3.6(2) \\
3.4(-1)\end{array}$ & $\begin{array}{l}2.6(3) \\
1.3(3) \\
9.1(-1)\end{array}$ & $\begin{array}{l}2.9(6) \\
9.0(3) \\
2.8(3)\end{array}$ & $\begin{array}{l}7 \\
7 \\
7\end{array}$ \\
\hline${ }^{37} \mathrm{Ar}$ & $\begin{array}{l}(n, y) \\
(\alpha, p) \\
(\alpha, n) \\
(\alpha, \gamma)\end{array}$ & 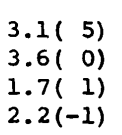 & 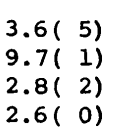 & $\begin{array}{l}1.3(0) \\
1.1(4) \\
8.2(4) \\
1.4(1)\end{array}$ & $\begin{array}{l}1.4(4) \\
5.6(4) \\
3.6(5) \\
3.2(4)\end{array}$ & $\begin{array}{l}7 \\
7 \\
7 \\
7\end{array}$ \\
\hline${ }^{38}{ }_{\mathrm{Ar}}$ & $\begin{array}{l}(p, \gamma) \\
(\alpha, p) \\
(\alpha, n) \\
(\alpha, y)\end{array}$ & $\begin{array}{l}1.2(4) \\
1.5(-1) \\
1.0(0) \\
6.0(0)\end{array}$ & $\begin{array}{l}2.4(4) \\
9.7(0) \\
6.7(1) \\
5.4(1)\end{array}$ & $\begin{array}{l}2.4(5) \\
8.8(4) \\
1.6(7) \\
2.7(3)\end{array}$ & $\begin{array}{l}8.0(7) \\
2.9(5) \\
2.1(7) \\
3.5(6)\end{array}$ & $\begin{array}{l}7 \\
7 \\
7 \\
7\end{array}$ \\
\hline${ }^{39} \mathrm{~K}$ & $\begin{array}{l}(n, \gamma) \\
(p, \gamma) \\
(\alpha, p) \\
(\alpha, \gamma)\end{array}$ & $\begin{array}{l}6.8(5) \\
3.3(3) \\
8.7(0) \\
4.4(-1)\end{array}$ & 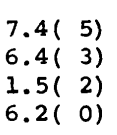 & $\begin{array}{l}2.2(5) \\
1.7(3) \\
1.9(2) \\
1.2(4)\end{array}$ & $\begin{array}{l}1.1(8) \\
2.2(6) \\
3.1(3) \\
8.6(6)\end{array}$ & $\begin{array}{l}7 \\
2 \\
7 \\
7\end{array}$ \\
\hline $40_{K}$ & $\begin{array}{l}(p, n) \\
(p, \gamma)\end{array}$ & $\begin{array}{l}1.5(5) \\
1.1(4)\end{array}$ & $\begin{array}{l}4.5(5) \\
2.4(4)\end{array}$ & $\begin{array}{l}2.3(5) \\
2.3(2)\end{array}$ & $\begin{array}{l}1.0(6) \\
5.3(5)\end{array}$ & $\begin{array}{l}7 \\
7\end{array}$ \\
\hline${ }^{41}{ }_{K}$ & $\begin{array}{l}(p, n) \\
(p, \gamma)\end{array}$ & $\begin{array}{l}2.5(5) \\
1.4(4)\end{array}$ & $\begin{array}{l}9.9(5) \\
2.9(4)\end{array}$ & $\begin{array}{l}7.0(6) \\
1.1(1)\end{array}$ & $\begin{array}{l}1.2(7) \\
6.5(4)\end{array}$ & $\begin{array}{l}7 \\
7\end{array}$ \\
\hline${ }^{40} \mathrm{Ca}$ & $\begin{array}{l}(n, \gamma) \\
(\alpha, p) \\
(\alpha, \gamma)\end{array}$ & $\begin{array}{l}1: 1(6) \\
4.8(-1) \\
1.4(0)\end{array}$ & $\begin{array}{l}1.2(6) \\
2.5(1) \\
1.6(1)\end{array}$ & $\begin{array}{l}1.5(4) \\
2.7(4) \\
1.8(4)\end{array}$ & $\begin{array}{l}1.2(7) \\
1.0(5) \\
1.5(7)\end{array}$ & $\begin{array}{l}7 \\
7 \\
7\end{array}$ \\
\hline${ }^{41} \mathrm{Ca}$ & $\begin{array}{l}(n, \gamma) \\
(\alpha, p) \\
(\alpha, n) \\
(\alpha, \gamma)\end{array}$ & $\begin{array}{l}1.2(6) \\
1.3(0) \\
9.1(-1) \\
8.5(-1)\end{array}$ & $\begin{array}{l}1.4(6) \\
3.9(1) \\
2.4(1) \\
1.2(1)\end{array}$ & 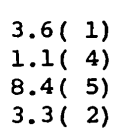 & $\begin{array}{l}2.9(5) \\
6.3(4) \\
2.2(6) \\
6.9(5)\end{array}$ & $\begin{array}{l}7 \\
7 \\
7 \\
7\end{array}$ \\
\hline${ }^{42} \mathrm{Ca}$ & $\begin{array}{l}(n, \gamma) \\
(p, \gamma) \\
(\alpha, p)+ \\
(\alpha, n) \\
(\alpha, \gamma)+\end{array}$ & $\begin{array}{l}1.8(6) \\
8.4(3) \\
1.4(0) \\
3.8(-1) \\
1.7(0)\end{array}$ & $\begin{array}{l}2.3(6) \\
2.0(4) \\
4.3(1) \\
2.5(1) \\
2.4(1)\end{array}$ & $\begin{array}{l}1.1(5) \\
1.1(7) \\
1.5(3) \\
4.9(6) \\
2.2(0)\end{array}$ & $\begin{array}{l}6.7(7) \\
1.4(9) \\
8.2(3) \\
7.1(6) \\
1.6(4)\end{array}$ & $\begin{array}{l}7 \\
7 \\
7 \\
7 \\
7\end{array}$ \\
\hline${ }^{43} \mathrm{Ca}$ & $\begin{array}{l}(n, \gamma) \\
(p, n) \\
(p, \gamma) \\
(\alpha, p) \\
(\alpha, n) \\
(\alpha, \gamma)\end{array}$ & $\begin{array}{l}2.0(6) \\
1.1(4) \\
3.5(4) \\
2.2(0) \\
2.5(0) \\
2.1(-1)\end{array}$ & $\begin{array}{l}2.3(6) \\
1.2(5) \\
8.0(4) \\
5.9(1) \\
4.9(1) \\
4.6(0)\end{array}$ & $\begin{array}{l}1.8(2) \\
2.3(8) \\
1.5(6) \\
1.2(3) \\
7.0(0) \\
9.7(-2)\end{array}$ & $\begin{array}{l}1.1(6) \\
2.7(8) \\
7.2(8) \\
1.0(4) \\
1.4(2) \\
2.2(3)\end{array}$ & $\begin{array}{l}7 \\
7 \\
7 \\
7 \\
7 \\
7\end{array}$ \\
\hline${ }^{44} \mathrm{Ca}$ & $\begin{array}{l}(p, n) \\
(p, \gamma) \\
(\alpha, n)\end{array}$ & $\begin{array}{l}3.0(2) \\
4.5(4) \\
5.4(0)\end{array}$ & $\begin{array}{l}9.0(3) \\
1.2(5) \\
1.2(2)\end{array}$ & $\begin{array}{l}1.4(8) \\
8.3(4) \\
4.5(3)\end{array}$ & $\begin{array}{l}1.7(8) \\
5.2(7) \\
2.0(4)\end{array}$ & $\begin{array}{l}7 \\
7 \\
7\end{array}$ \\
\hline${ }^{43} \mathrm{Sc}$ & $\begin{array}{l}(n, \gamma) \\
(p, \gamma) \\
(\alpha, p) \\
(\alpha, \gamma)\end{array}$ & $\begin{array}{l}2.5(6) \\
1.7(4) \\
1.7(0) \\
5.7(-1)\end{array}$ & $\begin{array}{l}3.2(6) \\
4.1(4) \\
3.6(1) \\
1.4(1)\end{array}$ & $\begin{array}{l}5.0(3) \\
3.8(3) \\
1.8(-3) \\
6.5(-1)\end{array}$ & $\begin{array}{l}1.2(7) \\
8.8(6) \\
3.7(-1) \\
1.0(4)\end{array}$ & $\begin{array}{l}7 \\
7 \\
7 \\
7\end{array}$ \\
\hline${ }^{44} \mathrm{Sc}$ & $\begin{array}{l}(n, \gamma) \\
(p, n) \\
(p, \gamma) \\
(\alpha, p) \\
(\alpha, n)\end{array}$ & $\begin{array}{l}1.1(7) \\
1.6(5) \\
1.8(4) \\
2.1(0) \\
1.6(0)\end{array}$ & $\begin{array}{l}1.4(7) \\
5.9(5) \\
4.5(4) \\
5.0(1) \\
4.0(1)\end{array}$ & $\begin{array}{l}4.1(1) \\
1.8(7) \\
8.3(2) \\
3.6(-3) \\
9.0(2)\end{array}$ & $\begin{array}{l}3.2(5) \\
3.3(7) \\
1.6(6) \\
4.5(-1) \\
7.7(3)\end{array}$ & $\begin{array}{l}7 \\
7 \\
7 \\
7 \\
7\end{array}$ \\
\hline
\end{tabular}


TABLE 13-Continued

\begin{tabular}{|c|c|c|c|c|c|c|}
\hline \multirow{3}{*}{ Target } & \multirow{3}{*}{ Reaction } & \multicolumn{4}{|c|}{$\mathbf{N}_{\mathrm{A}}<\sigma \mathbf{v}>$} & \multirow{3}{*}{ Ref. } \\
\hline & & \multicolumn{2}{|c|}{ Forward Rate } & \multicolumn{2}{|c|}{ Reverse Rate } & \\
\hline & & $\mathrm{T}_{9}=3.5$ & $T_{9}=4.5$ & $T_{9}=3.5$ & $T_{9}=4.5$ & \\
\hline${ }^{45} \mathrm{Sc}$ & $\begin{array}{l}(n, y) \\
(p, n)+ \\
(p, y)+\end{array}$ & $\begin{array}{l}5.1(6) \\
1.9(4) \\
4.9(4)\end{array}$ & $\begin{array}{l}5.7(6) \\
1.7(5) \\
8.7(4)\end{array}$ & $\begin{array}{l}1.3(5) \\
2.3(8) \\
6.1(1)\end{array}$ & $\begin{array}{l}1.4(8) \\
2.6(8) \\
3.3(5)\end{array}$ & $\begin{array}{l}7 \\
7 \\
2\end{array}$ \\
\hline${ }^{44} \mathrm{Ti}$ & $\begin{array}{l}(n, \gamma) \\
(\alpha, p) \\
(\alpha, \gamma)\end{array}$ & $\begin{array}{l}3.6(6) \\
1.1(0) \\
2.7(-2)\end{array}$ & $\begin{array}{l}4.1(6) \\
2.9(1) \\
7.1(-1)\end{array}$ & $\begin{array}{l}1.6(3) \\
5.2(0) \\
1.5(-1)\end{array}$ & $\begin{array}{l}2.6(6) \\
9.7(1) \\
1.5(3)\end{array}$ & $\begin{array}{l}7 \\
7 \\
7\end{array}$ \\
\hline${ }^{45} 5_{\mathrm{Ti}}$ & $\begin{array}{l}(n, \gamma)+ \\
(\alpha, p) \\
(\alpha, n)\end{array}$ & $\begin{array}{l}1.2(7) \\
9.0(-1) \\
1.9(-1)\end{array}$ & $\begin{array}{l}1.3(7) \\
2.5(1) \\
5.3(0)\end{array}$ & $\begin{array}{l}1.1(0) \\
3.9(-1) \\
2.4(3)\end{array}$ & $\begin{array}{l}3.2(4) \\
1.7(1) \\
1.7(4)\end{array}$ & $\begin{array}{l}7 \\
7 \\
7\end{array}$ \\
\hline${ }^{46} \mathrm{Ti}$ & $\begin{array}{l}(n, \gamma) \\
(p, \gamma) \\
(\alpha, p) \\
(\alpha, n) \\
(\alpha, \gamma)\end{array}$ & $\begin{array}{l}3.9(6) \\
1.5(4) \\
4.6(-1) \\
3.3(-1) \\
7.1(-2)\end{array}$ & $\begin{array}{l}5.1(6) \\
4.6(4) \\
1.4(1) \\
1.7(1) \\
1.6(0)\end{array}$ & $\begin{array}{l}1.3(4) \\
1.7(7) \\
6.6(0) \\
4.1(5) \\
1.6(-2)\end{array}$ & $\begin{array}{l}1.8(7) \\
3.3(9) \\
9.0(1) \\
8.3(5) \\
2.8(2)\end{array}$ & $\begin{array}{l}7 \\
7 \\
7 \\
7 \\
7\end{array}$ \\
\hline${ }^{47} \mathrm{Ti}$ & $\begin{array}{l}(n, \gamma) \\
(p, n) \\
(p, \gamma)\end{array}$ & $\begin{array}{l}8.8(6) \\
1.3(3) \\
5.2(4)\end{array}$ & $\begin{array}{l}9.8(6) \\
2.4(4) \\
1.4(5)\end{array}$ & $\begin{array}{l}1.2(2) \\
4.3(8) \\
6.3(5)\end{array}$ & $\begin{array}{l}1.0(6) \\
5.1(8) \\
3.9(8)\end{array}$ & $\begin{array}{l}7 \\
2 \\
7\end{array}$ \\
\hline${ }^{48} 8_{\mathrm{Ti}}$ & $\begin{array}{l}(p, n) \\
(p, \gamma)\end{array}$ & $\begin{array}{l}2.9(1) \\
3.6(4)\end{array}$ & $\begin{array}{l}1.3(3) \\
1.1(5)\end{array}$ & $\begin{array}{l}2.6(7) \\
1.0(5)\end{array}$ & $\begin{array}{l}3.3(7) \\
6.5(7)\end{array}$ & $\begin{array}{l}2 \\
7\end{array}$ \\
\hline${ }^{47} \mathrm{v}$ & $\begin{array}{l}(n, \gamma) \\
(p, \gamma)\end{array}$ & $\begin{array}{l}1.6(7) \\
2.8(4)\end{array}$ & $\begin{array}{l}2.0(7) \\
7.9(4)\end{array}$ & $\begin{array}{l}6.0(2) \\
3.1(4)\end{array}$ & $\begin{array}{l}2.6(6) \\
4.9(7)\end{array}$ & $\begin{array}{l}7 \\
7\end{array}$ \\
\hline${ }^{48} \mathrm{v}$ & $\begin{array}{l}(n, \gamma) \\
(p, n) \\
(p, \gamma)\end{array}$ & $\begin{array}{l}1.2(7) \\
1.9(3) \\
2.8(4)\end{array}$ & $\begin{array}{l}1.5(7) \\
1.5(4) \\
8.1(4)\end{array}$ & $\begin{array}{l}3.9(1) \\
5.5(7) \\
7.9(3)\end{array}$ & $\begin{array}{l}3.5(5) \\
7.4(7) \\
1.4(7)\end{array}$ & $\begin{array}{l}7 \\
7 \\
7\end{array}$ \\
\hline $49 \mathrm{v}$ & $\begin{array}{l}(n, \gamma) \\
(p, n) \\
(p, \gamma)\end{array}$ & $\begin{array}{l}9.8(6) \\
2.8(3) \\
3.4(4)\end{array}$ & $\begin{array}{l}1.1(7) \\
3.9(4) \\
9.5(4)\end{array}$ & $\begin{array}{l}2.9(4) \\
2.5(8) \\
5.3(2)\end{array}$ & $\begin{array}{l}4.6(7) \\
2.9(8) \\
2.5(6)\end{array}$ & $\begin{array}{l}7 \\
7 \\
7\end{array}$ \\
\hline${ }^{50} \mathrm{v}$ & $\begin{array}{l}(p, n) \\
(p, \gamma)\end{array}$ & $\begin{array}{l}4.2(3) \\
1.8(4)\end{array}$ & $\begin{array}{l}2.0(4) \\
5.8(4)\end{array}$ & $\begin{array}{l}2.2(4) \\
6.7(1)\end{array}$ & $\begin{array}{l}1.2(5) \\
3.4(5)\end{array}$ & $\begin{array}{l}7 \\
7\end{array}$ \\
\hline${ }^{48} \mathrm{Cr}$ & $\begin{array}{l}(n, \gamma) \\
(\alpha, p) \\
(\alpha, \gamma)\end{array}$ & $\begin{array}{l}1.2(7) \\
2.7(-1) \\
1.8(-3)\end{array}$ & $\begin{array}{l}1.4(7) \\
9.3(0) \\
6.9(-2)\end{array}$ & $\begin{array}{l}1.1(2) \\
2.3(-2) \\
2.9(-3)\end{array}$ & $\begin{array}{l}5.0(5) \\
1.3(0) \\
5.8(1)\end{array}$ & $\begin{array}{l}7 \\
7 \\
7\end{array}$ \\
\hline${ }^{49} \mathrm{Cr}$ & $\begin{array}{l}(n, \gamma) \\
(\alpha, p) \\
(\alpha, n)\end{array}$ & $\begin{array}{l}9.9(6) \\
1.4(-1) \\
1.2(-1)\end{array}$ & $\begin{array}{l}1.2(7) \\
5.0(0) \\
4.4(0)\end{array}$ & $\begin{array}{l}1.8(0) \\
5.8(-2) \\
2.1(4)\end{array}$ & $\begin{array}{l}4.1(4) \\
2.9(0) \\
1.0(5)\end{array}$ & $\begin{array}{l}7 \\
7 \\
7\end{array}$ \\
\hline${ }^{50} \mathrm{Cr}$ & $\begin{array}{l}(n, \gamma) \\
(p, \gamma) \\
(\alpha, p)\end{array}$ & $\begin{array}{l}7.8(6) \\
7.2(3) \\
2.2(-1)\end{array}$ & $\begin{array}{l}9.9(6) \\
2.3(4) \\
7.3(0)\end{array}$ & $\begin{array}{l}5.4(3) \\
3.5(6) \\
4.2(-1)\end{array}$ & $\begin{array}{l}9.3(6) \\
8.1(8) \\
1.0(1)\end{array}$ & $\begin{array}{l}7 \\
7 \\
7\end{array}$ \\
\hline${ }^{51} \mathrm{Cr}$ & $\begin{array}{l}(p, n) \\
(p, y)\end{array}$ & $\begin{array}{l}4.6(2) \\
1.8(4)\end{array}$ & $\begin{array}{l}9.8(3) \\
5.2(4)\end{array}$ & $\begin{array}{l}3.2(8) \\
5.7(5)\end{array}$ & $\begin{array}{l}3.7(8) \\
3.0(8)\end{array}$ & $\begin{array}{l}7 \\
7\end{array}$ \\
\hline $51_{\mathrm{Mn}}$ & $\begin{array}{l}(n, \gamma) \\
(p, \gamma) \\
(\alpha, p)\end{array}$ & $\begin{array}{l}7.1(6) \\
5.6(3) \\
1.3(-1)\end{array}$ & $\begin{array}{l}8.5(6) \\
1.5(4) \\
4.6(0)\end{array}$ & $\begin{array}{l}3.2(2) \\
1.1(5) \\
9.0(-5)\end{array}$ & $\begin{array}{l}1.3(6) \\
9.7(7) \\
3.2(-2)\end{array}$ & $\begin{array}{l}7 \\
7 \\
7\end{array}$ \\
\hline $52_{\mathrm{Mn}}$ & $\begin{array}{l}(n, \gamma) \\
(p, n) \\
(p, \gamma)\end{array}$ & $\begin{array}{l}4.5(6) \\
1.3(1) \\
3.4(3)\end{array}$ & $\begin{array}{l}5.4(6) \\
2.5(2) \\
1.1(4)\end{array}$ & $\begin{array}{l}3.7(0) \\
5.6(6) \\
2.1(4)\end{array}$ & $\begin{array}{l}4.7(4) \\
1.0(7) \\
2.0(7)\end{array}$ & $\begin{array}{l}7 \\
7 \\
7\end{array}$ \\
\hline${ }^{53} \mathrm{Mn}$ & $\begin{array}{l}(p, n) \\
(p, \gamma)\end{array}$ & $\begin{array}{l}4.5(1) \\
1.4(4)\end{array}$ & $\begin{array}{l}1.8(3) \\
4.2(4)\end{array}$ & $\begin{array}{l}3.3(8) \\
2.5(3)\end{array}$ & $\begin{array}{l}4.0(8) \\
7.4(6)\end{array}$ & $\begin{array}{l}7 \\
7\end{array}$ \\
\hline
\end{tabular}

308 
TABLE 13-Continued

\begin{tabular}{|c|c|c|c|c|c|c|}
\hline \multirow{3}{*}{ Target } & \multirow{3}{*}{ Reaction } & \multicolumn{4}{|c|}{$\left.\mathrm{N}_{\mathrm{A}}<\sigma \mathrm{v}\right\rangle$} & \multirow{3}{*}{ Ref. } \\
\hline & & \multicolumn{2}{|c|}{ Forward Rate } & \multicolumn{2}{|c|}{ Reverse Rate } & \\
\hline & & $\mathrm{T}_{9}=3.5$ & $\mathrm{~T}_{9}=4.5$ & $T_{9}=3.5$ & $\mathrm{~T}_{9}=4.5$ & \\
\hline${ }^{52} \mathrm{Fe}$ & $\begin{array}{l}(n, \gamma) \\
(\alpha, p) \\
(\alpha, \gamma)\end{array}$ & $\begin{array}{l}8.7(6) \\
5.3(-2) \\
2.4(-4)\end{array}$ & $\begin{array}{l}1.0(7) \\
2.2(0) \\
1.1(-2)\end{array}$ & $\begin{array}{l}1.3(2) \\
1.6(-3) \\
3.3(-4)\end{array}$ & $\begin{array}{l}4.7(5) \\
1.2(-1) \\
8.1(0)\end{array}$ & $\begin{array}{l}7 \\
7 \\
7\end{array}$ \\
\hline${ }^{53} \mathrm{Fe}$ & $\begin{array}{l}(n, \gamma) \\
(\alpha, p)\end{array}$ & $\begin{array}{l}4.8(6) \\
5.4(-2)\end{array}$ & $\begin{array}{l}6.3(6) \\
2.4(0)\end{array}$ & $\begin{array}{l}1.2(-1) \\
3.7(-2)\end{array}$ & $\begin{array}{l}5.0\left(3^{3}\right) \\
2.3(0)\end{array}$ & $\begin{array}{l}7 \\
7\end{array}$ \\
\hline${ }^{54} \mathrm{Fe}$ & $\begin{array}{l}(n, \gamma) \\
(p, \gamma) \\
(\alpha, p) \\
(\alpha, n) \\
(\alpha, \gamma)\end{array}$ & $\begin{array}{l}5.6(6) \\
3.9(3) \\
3.6(-2) \\
8.9(-3) \\
3.9(-3)\end{array}$ & $\begin{array}{l}7.0(6) \\
1.2(4) \\
1.8(0) \\
1.1(0) \\
1.0(-1)\end{array}$ & $\begin{array}{l}7.1(3) \\
3.1(6) \\
5.9(0) \\
1.8(6) \\
1.1(0)\end{array}$ & $\begin{array}{l}1.2(7) \\
6.1(8) \\
7.8(1) \\
3.1(6) \\
4.6(3)\end{array}$ & $\begin{array}{l}7 \\
7 \\
7 \\
7 \\
7\end{array}$ \\
\hline $55_{\mathrm{Fe}}$ & $\begin{array}{l}(n, \gamma) \\
(p, n) \\
(p, \gamma)\end{array}$ & $\begin{array}{l}8.3(6) \\
2.0(2) \\
8.5(3)\end{array}$ & $\begin{array}{l}9.5(6) \\
5.5(3) \\
2.5(4)\end{array}$ & $\begin{array}{l}3.0(2) \\
1.3(8) \\
1.8(6)\end{array}$ & $\begin{array}{l}2.0(6) \\
1.6(8) \\
5.8(8)\end{array}$ & $\begin{array}{l}7 \\
7 \\
7\end{array}$ \\
\hline${ }^{56} \mathrm{Fe}$ & $\begin{array}{l}(p, n) \\
(p, \gamma)\end{array}$ & $\begin{array}{l}6.7\left(\begin{array}{l}0 \\
6.8(3)\end{array}\right)\end{array}$ & $\begin{array}{l}4.1(2) \\
2.3(4)\end{array}$ & $\begin{array}{l}3.9\left(\begin{array}{l}7 \\
2.3(5)\end{array}\right.\end{array}$ & $\begin{array}{l}4.6(7) \\
9.2(7)\end{array}$ & $\begin{array}{l}7 \\
7\end{array}$ \\
\hline${ }^{55} \mathrm{Co}$ & $\begin{array}{l}(n, \gamma) \\
(p, \gamma) \\
(\alpha, p)\end{array}$ & $\begin{array}{l}3.7(6) \\
2.5(3) \\
3.0(-2)\end{array}$ & $\begin{array}{l}4.4(6) \\
6.8(3) \\
1.4(0)\end{array}$ & $\begin{array}{l}1.2(3) \\
1.2(5) \\
1.0(-2)\end{array}$ & $\begin{array}{l}3.6(6) \\
9.2(7) \\
1.2(0)\end{array}$ & $\begin{array}{l}7 \\
7 \\
7\end{array}$ \\
\hline${ }^{56} \mathrm{Co}$ & $\begin{array}{l}(n, \gamma) \\
(p, n) \\
(p, \gamma)\end{array}$ & $\begin{array}{l}6.9(6) \\
2.7(2) \\
5.1(3)\end{array}$ & $\begin{array}{l}7.8(6) \\
3.6(3) \\
1.5(4)\end{array}$ & $\begin{array}{l}3.9(1) \\
3.9(7) \\
3.6(4)\end{array}$ & $\begin{array}{l}2.9(5) \\
6.0(7) \\
3.5(7)\end{array}$ & $\begin{array}{l}7 \\
7 \\
7\end{array}$ \\
\hline${ }^{57} \mathrm{Co}$ & $\begin{array}{l}(p, n) \\
(p, \gamma)\end{array}$ & $\begin{array}{l}1.4(2) \\
7.5(3)\end{array}$ & $\begin{array}{l}3.4(3) \\
2.3(4)\end{array}$ & $\begin{array}{l}1.7(8) \\
1.3(4)\end{array}$ & $\begin{array}{l}2.2(8) \\
2.3(7)\end{array}$ & $\begin{array}{l}7 \\
7\end{array}$ \\
\hline${ }^{56} \mathrm{Ni}$ & $\begin{array}{l}(n, \gamma) \\
(\alpha, p) \dagger \\
(\alpha, \gamma) \neq\end{array}$ & $\begin{array}{l}3.2(6) \\
7.9(-2) \\
4.8(-4)\end{array}$ & $\begin{array}{l}3.7(6) \\
3.4(0) \\
1.3(-2)\end{array}$ & $\begin{array}{l}1.6(2) \\
2.1(2) \\
2.8(4)\end{array}$ & $\begin{array}{l}5.2(5) \\
1.5(3) \\
8.1(6)\end{array}$ & $\begin{array}{l}7 \\
7 \\
7\end{array}$ \\
\hline${ }^{57} \mathrm{Ni}$ & $\begin{array}{l}(n, \gamma) \\
(\alpha, p)+ \\
(\alpha, \gamma)+\end{array}$ & $\begin{array}{l}2.8(6) \\
4.9(-2) \\
3.8(-3)\end{array}$ & $\begin{array}{l}3.4(6) \\
2.8(0) \\
8.6(-2)\end{array}$ & $\begin{array}{l}3.8(0) \\
8.7(2) \\
1.2(5)\end{array}$ & $\begin{array}{l}5.5(4) \\
7.1(3) \\
3.3(7)\end{array}$ & $\begin{array}{l}7 \\
7 \\
7\end{array}$ \\
\hline${ }^{58} \mathrm{Ni}$ & $\begin{array}{l}(n, \gamma) \\
(p, \gamma)+ \\
(\alpha, p)+ \\
(\alpha, \gamma)+\end{array}$ & $\begin{array}{l}7.0(6) \\
9.3(2) \\
1.0(-2) \\
2.8(-3)\end{array}$ & $\begin{array}{l}8.7(6) \\
3.0(3) \\
8.2(-1) \\
1.0(-1)\end{array}$ & $\begin{array}{l}2.4(4) \\
3.5(8) \\
2.8(2) \\
2.2(4)\end{array}$ & $\begin{array}{l}3.3(7) \\
2.1(10) \\
2.3(3) \\
1.4(7)\end{array}$ & $\begin{array}{l}7 \\
7 \\
7 \\
7\end{array}$ \\
\hline${ }^{59}{ }_{\mathrm{Ni}}$ & $\begin{array}{l}(p, n) \\
(p, \gamma)\end{array}$ & $\begin{array}{l}4.3(0) \\
3.5(3)\end{array}$ & $\begin{array}{l}3.0(2) \\
1.2(4)\end{array}$ & $\begin{array}{l}4.8(8) \\
1.3(8)\end{array}$ & $\begin{array}{l}5.4(8) \\
1.7(10)\end{array}$ & $\begin{array}{l}7 \\
7\end{array}$ \\
\hline${ }^{59} \mathrm{Cu}$ & $\begin{array}{l}(n, \gamma) \\
(p, \gamma)+ \\
(\alpha, p)\end{array}$ & $\begin{array}{l}3.1(6) \\
4.7(1) \\
1.1(-2)\end{array}$ & $\begin{array}{l}4.2(6) \\
1.6(2) \\
6.0(-1)\end{array}$ & $\begin{array}{l}1.0(3) \\
1.0(6) \\
2.2(-1)\end{array}$ & $\begin{array}{l}3.3(6) \\
2.2(8) \\
1.2(1)\end{array}$ & $\begin{array}{l}7 \\
7 \\
7\end{array}$ \\
\hline${ }^{60} \mathrm{Cu}$ & $\begin{array}{l}(n, \gamma) \\
(p, n) \\
(p, \gamma)+ \\
(\alpha, p)\end{array}$ & $\begin{array}{l}1.2(7) \\
3.2(0) \\
1.5(3) \\
2.1(-2)\end{array}$ & $\begin{array}{l}1.5(7) \\
2.0(2) \\
4.5(3) \\
1.0(0)\end{array}$ & $\begin{array}{l}2.6(1) \\
2.1(8) \\
2.6(6) \\
2.7(0)\end{array}$ & $\begin{array}{l}2.6(5) \\
3.4(8) \\
6.7(8) \\
6.0(1)\end{array}$ & $\begin{array}{l}7 \\
7 \\
7 \\
7\end{array}$ \\
\hline${ }^{61} \mathrm{Cu}$ & $\begin{array}{l}(p, n) \\
(p, \gamma)+ \\
(\alpha, p)\end{array}$ & $\begin{array}{l}5.6(-1) \\
3.3(3) \\
4.5(-2)\end{array}$ & $\begin{array}{l}6.1(1) \\
1.2(4) \\
2.1(0)\end{array}$ & $\begin{array}{l}4.6(8) \\
9.1(5) \\
1.1(1)\end{array}$ & $\begin{array}{l}5.2(8) \\
5.5(8) \\
2.8(2)\end{array}$ & $\begin{array}{l}7 \\
7 \\
7\end{array}$ \\
\hline${ }^{60} \mathrm{Zn}$ & $\begin{array}{l}(n, \gamma) \\
(\alpha, p)+ \\
(\alpha, \gamma)\end{array}$ & $\begin{array}{l}4.9(6) \\
3.4(-2) \\
2.0(-5)\end{array}$ & $\begin{array}{l}5.7(6) \\
2.0(0) \\
8.9(-4)\end{array}$ & $\begin{array}{l}1.3(2) \\
7.2(1) \\
5.0(3)\end{array}$ & $\begin{array}{l}4.9(5) \\
7.4(2) \\
1.8(6)\end{array}$ & $\begin{array}{l}7 \\
7 \\
7\end{array}$ \\
\hline${ }^{61} \mathrm{zn}$ & $\begin{array}{l}(n, \gamma) \\
(\alpha, p) \\
(\alpha, n) \\
(\alpha, \gamma)\end{array}$ & $\begin{array}{l}5.5(6) \\
1.5(-2) \\
2.6(-6) \\
2.2(-4)\end{array}$ & $\begin{array}{l}7.6(6) \\
1.0(0) \\
2.5(-3) \\
8.9(-3)\end{array}$ & $\begin{array}{l}1.9(0) \\
9.7(2) \\
2.4(7) \\
9.5(4)\end{array}$ & $\begin{array}{l}4.1(4) \\
1.0(4) \\
5.6(7) \\
2.7(7)\end{array}$ & $\begin{array}{l}7 \\
7 \\
7 \\
7\end{array}$ \\
\hline
\end{tabular}

309 
TABLE 13-Continued

\begin{tabular}{|c|c|c|c|c|c|c|}
\hline \multirow{3}{*}{ Target } & \multirow{3}{*}{ Reaction } & \multicolumn{4}{|c|}{$N_{A}\langle 0 v\rangle$} & \multirow{3}{*}{ Ref. } \\
\hline & & \multicolumn{2}{|c|}{ Forward Rate } & \multicolumn{2}{|c|}{ Reverse Rate } & \\
\hline & & $T_{9}=3.5$ & $T_{9}=4.5$ & $\mathrm{~T}_{9}=3.5$ & $T_{9}=4.5$ & \\
\hline${ }^{62} \mathrm{zn}$ & $\begin{array}{l}(n, \gamma) \\
(p, \gamma) \\
(\alpha, p) \\
(\alpha, \gamma)\end{array}$ & $\begin{array}{l}9.9(6) \\
1.1(3) \\
2.1(-2) \\
3.2(-3)\end{array}$ & 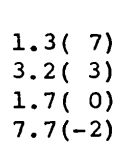 & $\begin{array}{l}2.0(4) \\
2.3(9) \\
1.2(3) \\
1.7(6)\end{array}$ & $\begin{array}{l}3.1(7) \\
8.5(10) \\
8.4(3) \\
2.7(8)\end{array}$ & $\begin{array}{l}7 \\
7 \\
7 \\
7\end{array}$ \\
\hline${ }^{63} \mathrm{zn}$ & $(p, \gamma)$ & $1.7(3)$ & $5.3(3)$ & $1.4(9)$ & $1.3(11)$ & 7 \\
\hline${ }^{63} \mathrm{Ga}$ & $(p, \gamma)+$ & $1.8(2)$ & $5.3(2)$ & $2.8(7)$ & $3.3(9)$ & 7 \\
\hline${ }^{64} \mathrm{Ga}$ & $\begin{array}{l}(p, n) \\
(p, \gamma)+\end{array}$ & $\begin{array}{l}3.5(-1) \\
5.5(3)\end{array}$ & $\begin{array}{l}5.3(1) \\
1.8(4)\end{array}$ & $\begin{array}{l}5.1(7) \\
3.8(7)\end{array}$ & $\begin{array}{l}1.2(8) \\
5.5(9)\end{array}$ & $\begin{array}{l}7 \\
7\end{array}$ \\
\hline${ }^{65} \mathrm{Ga}$ & $\begin{array}{l}(p, n) \\
(p, y)\end{array}$ & $\begin{array}{l}2.9(-2) \\
7.4(2)\end{array}$ & $\begin{array}{l}6.3\left(\begin{array}{l}0 \\
2.4( \\
2.4\end{array}\right)\end{array}$ & $\begin{array}{l}6.4(8) \\
6.8(6)\end{array}$ & $\begin{array}{l}7.0(8) \\
1.7(9)\end{array}$ & $\begin{array}{l}7 \\
7\end{array}$ \\
\hline $64_{\mathrm{Ge}}$ & $(n, \gamma)$ & $9.6(6)$ & $1.2(7)$ & $4.7(2)$ & $1.7(6)$ & 7 \\
\hline
\end{tabular}

\section{NOTES TO TABLE 13}

* All strong nuclear reactions shown in fig. 1 but not listed here were deemed to be of only secondary interest. The rates for all these unlisted reactions were taken from source (7).

$\dagger$ Reaction rate very important to the nucleosynthesis which occurs during explosive oxygen or silicon burning.

1. The rate given is the rate of formation of the compound nucleus.

2. Fowler et al. $(1967,1971)$.

3. Patterson, Winkler, and Zaidins (1969) and Arnett and Truran (1969).

4. Patterson et al. (1971) and Woosley et al. (1972a).

5. Wagoner (1969).

6. Spinka and Winkler (1972) and TA70.

7. Truran et al. (1966) and Truran (1971).

error at $T_{9}=3.2$ would have been a factor of 5.59 , but since ${ }^{57} \mathrm{Ni}$ has a much higher first excited state than ${ }^{57} \mathrm{Fe}$, the actual error here turns out to be only 11 percent.

In most cases the inclusion of excited states in our calculations would have acted to give better agreement with Cameron (1968) abundances than shown in figure 25. The species ${ }^{49} \mathrm{Cr}\left({ }^{49} \mathrm{Ti}\right),{ }^{50} \mathrm{Cr},{ }^{51} \mathrm{Mn}\left({ }^{51} \mathrm{~V}\right)$, and ${ }^{52} \mathrm{Fe}\left({ }^{52} \mathrm{Cr}\right)$ which would be most increased (according to the above prescription) by including excited states were previously all underproduced.

For temperatures below $T_{9} \simeq 3.2$ the changes in abundances depend somewhat upon the values of the cross-sections themselves. There does not exist a simple prescription for simulating the effects of excited states upon the nuclear cross-section in a nonequilibrium situation. However, we may note: (1) the changes in abundance below $T_{9} \simeq 3.2$ are generally small (see table 3 ), (2) as the temperature decreases the effects of excited states are diminished, and (3) even though silicon quasi-equilibrium ceases to be a good approximation below $T_{9} \simeq 3.2$, a form of cluster equilibrium is still maintained down to about $T_{9}=2.5$, below which abundances change very little. Clusters usually contain an element of high abundance (and high first excited state) in rough equilibrium with several elements of lower abundance and low first excited 
states (see fig. $6 a$ ). This equilibrium character means that, even at temperatures a good bit less than $T_{9}=3.2$, the abundance of such a species still depends more upon its partition function and binding energy than upon the actual value of the cross-sections employed. Hence we predict that, unless the inclusion of excited states radically changes the cross-section itself (which it may), the effect of including excited states in explosive oxygen and silicon burning can be roughly estimated by calculating the ratio $G\left(T_{9}\right) / W_{0}$ at some temperature between 2.5 and 3.5 billion degrees. The maximum effect, as noted previously, is then about 60 percent and acts to improve the results of our calculations.

Another effect of excited states is that, by changing the average cross-sections and equilibrium abundance ratios, they alter the time scales for the various nuclear developments. Because most of the species which govern the major burning reactions; e.g., ${ }^{12} \mathrm{C},{ }^{16} \mathrm{O}$, and ${ }^{24} \mathrm{Mg}$, have high first excited states we expect that the effect of their inclusion upon the burning time scale would be very small. The effect of excited states upon equilibration time scales may be a good deal larger, but we do not feel that the accuracy of our adopted rates warrants such considerations at the present time. We have had to rely overwhelmingly on calculated reaction rates, and the uncertainty in most of these is probably greater than the modification of the rate due to excited states.

These extensive closing remarks have been offered to clarify our neglect of excited states in this study. By leaving them out we make only a formal omission. The physical conclusions are basically unaltered at the present level of numerical accuracy.

\section{REFERENCES}

Arnett, W. D. 1969a, Ap. and Space Sci., 5, 180.

$$
\text { 1969b, Ap. J., 157, } 1369 .
$$

1971, ibid., 166, 153.

1972, ibid., 173, 393.

Arnett, W. D., and Clayton, D. D. 1970, Nature, 227, 780.

Arnett, W. D., and Truran, J. W. 1969, Ap. J., 157, 339.

Arnett, W. D., Truran, J. W., and Woosley, S. E. 1971, Ap. J., 165, 87 (ATW).

Austin, S. M., Trentelman, G. F., and Kashy, E. 1971, Ap. J. (Letters), 163, L79.

Bodansky, D., Clayton, D. D., and Fowler, W. A. 1968, Ap. J. Suppl., No. 148, 16, 299 (BCF).

Burbidge, E. M., Burbidge, G. R., Fowler, W. A., and Hoyle, F. 1957, Rev. Mod. Phys., $29,547$.

Cameron, A. G. W. 1968, in The Origin and Distribution of the Elements, ed. L. H. Ahrens (New

York: Pergamon Press).

Clayton, D. D. 1971, Nature, 234, 291.

Clayton, D. D., Colgate, S. A., and Fishman, G. J. 1969, Ap. J., 155, 75.

Clayton, D. D., and Craddock, W. L. 1965, Ap. J., 142, 189.

Clayton, D. D., and Silk, J. I. 1969, Ap. J. (Letters), 158, L43.

Clayton, D. D., and Woosley, S. E. 1969, Ap. J., 157, 1381.

Crawford, H. J., Price, P. B., and Sullivan, J. D. 1972, Ap. J. (Letters), 175, L149.

Eberhardt, P., Geiss, J., Graf, H., Grögler, N., Krähenbuhl, U., Schwaller, H., Schwarlzmüller, J., and Stettler, A. 1970, in Proc. Apollo 11 Lunar Sci. Conference, ed. A. A. Levinson (New York: Pergamon Press), Vol. 2, 1037.

Fowler, W. A., Caughlan, G. R., and Zimmerman, B. A. 1967, Ann. Rev. Astr. and Ap., 5, 525.

. 1971, private communication.

Fowler, W. A., and Hoyle, F. 1964, Ap. J. Suppl., No. 91, 9, 201.

Fowler, W. A., and Vogl, J. L. 1964, in Lectures in Theoretical Physics (Boulder: University of Colorado Press), Vol. 6, 379.

Garz, G., and Kock, M. 1969, Astr. and Ap., $2,274$.

Grasdalen, G. L., Huber, M., and Parkinson, W. H. 1969, Ap. J., 156, 1153.

Hansen, C. J. 1967, Ph.D. dissertation, Yale University, New Haven. 1968, Ap. and Space Sci., 1, 499.

Hoyle, F. 1946, M.N.R.A.S., 106, 343.

Howard, W. M., Arnett, W. D., Clayton, D. D., and Woosley, S. E. 1972, Ap. J., 175, 201.

Huber, M., and Tobey, F. L. 1968, Ap. J., 152, 609.

Mattauch, J. H. E., Thiele, W., and Wapstra, A. H. 1965, Nucl. Phys., 67, 1.

Michaud, G., and Fowler, W. A. 1972. Ap. J., 173, 157.

Patterson, J. R., Nagorcka, B. N., Symons, G. D., and Zuk, W. M. 1971, Nucl. Phys., A165, 545. 
Patterson, J. R., Winkler, H., and Zaidins, C. S. 1969, Ap. J., 157, 367.

Salpeter, E. E., and Van Horn, H. M. 1969, Ap. J., 155, 183.

Spinka, H., and Winkler, H. 1972, Ap. J., 174, 455.

Talbot, R. J., and Arnett, W. D. 1971, Ap. J., 170, 409.

Truran, J. W. 1968a, Ap. and Space Sci., 2, 384.

-. 1968b, ibid., p. 391.

1971, private communication.

Truran, J. W., and Arnett, W. D. 1970, Ap. J., 160, 181 (TA70).

. 1971, Ap. and Space Sci., 11, 430.

Truran, J. W., Cameron, A. G. W., and Gilbert, A. 1966, Canadian J. Phys., 44, 563.

Truran, J. W., Hansen, C. J., Cameron, A. G. W., and Gilbert, A. 1966, Canadian J. Phys., 44, 151.

Wagoner, R. V. 1969, Ap. J. Suppl., No. 162, 18, 247.

Whaling, W., King, R. B., and Martinez-Garcia, M. 1969, Ap. J., 158, 389.

Woosley, S. E., Arnett, W. D., and Clayton, D. D. 1971, Phys. Rev. (Letters), 27, 213. 1972a, Phys. Letters, 38B, 196. 1972b, Ap. J., 175, 731 . 